JOINT TRANSPORTATION RESEARCH PROGRAM

FHWA/IN/JTRP-2005/27

Final Report

GUIDELINES FOR DATA COLLECTION

TECHNIQUES AND METHODS FOR ROADSIDE STATION ORIGIN-DESTINATION STUDIES

Bryan P. Guy

Jon D. Fricker

December 2005 
Final Report

FHWA/IN/JTRP-2005/27

\title{
GUIDELINES FOR DATA COLLECTION TECHNIQUES AND METHODS FOR ROADSIDE STATION ORIGIN-DESTINATION STUDIES
}

\author{
By \\ Bryan P. Guy \\ Research Assistant \\ School of Civil Engineering \\ Purdue University \\ and \\ Jon D. Fricker \\ Principal Investigator \\ Professor \\ School of Civil Engineering \\ Purdue University \\ Joint Transportation Research Program \\ Project No. C-36-17SSS \\ File No. 8-4-71 \\ SPR-2935 \\ Conducted in cooperation with the \\ Indiana Department of Transportation and \\ the U.S. Department of Transportation \\ Federal Highway Administration
}

The contents of this paper reflect the views of the authors, who are responsible for the facts and accuracy of the data presented herein, and do not necessarily reflect the official views or policies of the Federal Highway Administration and the Indiana Department of Transportation, nor do the contents constitute a standard, specification, or regulation.

Purdue University

West Lafayette, Indiana

December 2005 
TECHNICAL REPORT STANDARD TITLE PAGE

\begin{tabular}{|c|c|c|}
\hline $\begin{array}{l}\text { 1. } \text { Report No. } \\
\text { FHWA/IN/JTRP-2005/27 }\end{array}$ & 2. Government Accession No. & 3. Recipient's Catalog No. \\
\hline \multirow{2}{*}{\multicolumn{2}{|c|}{$\begin{array}{l}\text { 4. Title and Subtitle } \\
\text { Guidelines for Data Collection Techniques and Methods for Roadside Station } \\
\text { Origin-Destination Studies }\end{array}$}} & $\begin{array}{l}\text { 5. } \quad \text { Report Date } \\
\text { December } 2005\end{array}$ \\
\hline & & 6. Performing Organization Code \\
\hline \multicolumn{2}{|l|}{$\begin{array}{l}\text { 7. Author(s) } \\
\text { Bryan Guy and Jon Fricker }\end{array}$} & $\begin{array}{l}\text { 8. Performing Organization Report No. } \\
\text { FHWA/IN/JTRP-2005/27 }\end{array}$ \\
\hline \multirow{2}{*}{\multicolumn{2}{|c|}{$\begin{array}{l}\text { 9. Performing Organization Name and Address } \\
\text { Joint Transportation Research Program } \\
1284 \text { Civil Engineering Building } \\
\text { Purdue University } \\
\text { West Lafayette, IN 47907-1284 }\end{array}$}} & 10. Work Unit No. \\
\hline & & $\begin{array}{l}\text { 11. Contract or Grant No. } \\
\text { SPR-2935 }\end{array}$ \\
\hline \multirow{2}{*}{\multicolumn{2}{|c|}{$\begin{array}{l}\text { 12. Sponsoring Agency Name and Address } \\
\text { Indiana Department of Transportation } \\
\text { State Office Building } \\
100 \text { North Senate Avenue } \\
\text { Indianapolis, IN } 46204\end{array}$}} & $\begin{array}{l}\text { 13. Type of Report and Period Covered } \\
\text { Final Report }\end{array}$ \\
\hline & & 14. Sponsoring Agency Code \\
\hline \multicolumn{3}{|c|}{$\begin{array}{l}\text { 15. Supplementary Notes } \\
\text { Prepared in cooperation with the Indiana Department of Transportation and Federal Highway Administration. }\end{array}$} \\
\hline \multicolumn{3}{|c|}{$\begin{array}{l}\text { 16. Abstract } \\
\text { Origin-Destination studies are often used in transportation planning to determine the travel patterns (origin-destination } \\
\text { matrix) of vehicles and goods in a particular area. Given these travel patterns, the impacts of alternative solutions to current } \\
\text { and future transportation problems can be evaluated. Therefore, it is important that the travel patterns be accurately } \\
\text { measured. However, it is not always clear what data collection method should be used to obtain the type of data needed, } \\
\text { while maximizing quality and minimizing the time and cost. The objective of this research is to review both conventional } \\
\text { and experimental techniques for roadside station OD studies, and make general recommendations for the best OD study } \\
\text { technique and data collection method, given the roadway characteristics and traffic conditions. }\end{array}$} \\
\hline
\end{tabular}

origin-destination study, license plate matching, follow-up survey, vehicle intercept survey, roadside interview, global positioning system, wireless phone tracing, video license plate data collection, identification, recording, transcription, accuracy
18. Distribution Statement

No restrictions. This document is available to the public through the National Technical Information Service, Springfield, VA 22161

\begin{tabular}{|c|c|c|c|}
\hline 19. Security Classif. (of this report) & 20. Security Classif. (of this page) & 21. No. of Pages & 133 \\
Unclassified & Unclassified & Price & \\
\hline
\end{tabular}




\section{ACKNOWLEDGMENTS}

A few people deserve thanks for their contributions to the completion of this project. The authors would like to thank the study advisory committee members including Steve Smith, Rebecca Black, Roy Nunnally, Scott MacArthur, and Shuo Li of the Indiana Department of Transportation and Joyce Newland of the Federal Highway Administration for their valuable assistance and guidance. Also thanks to Karen Hatke of the Joint Transportation Research Program for her help in putting this report together. 


\section{TABLE OF CONTENTS}

Page

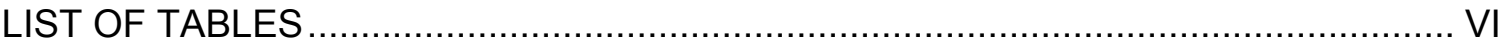

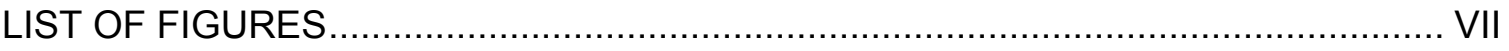

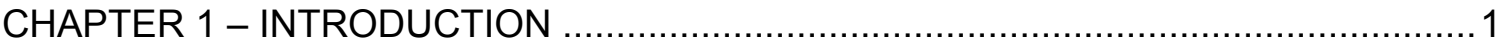

1.1 HOUSEHOLD TRAVEL SURVEYS (TRAVEL DIARIES) ..................................... 5

1.1.1 Example: Building a Travel Demand Model................................................ 6

1.1.2 Example: Updating a Travel Demand Model.......................................... 7

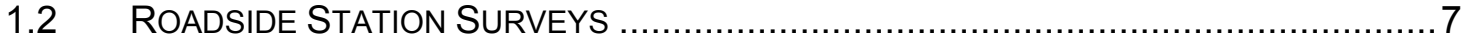

1.2.1 Example: Bypass Feasibility Study ................................................. 8

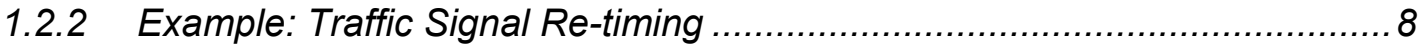

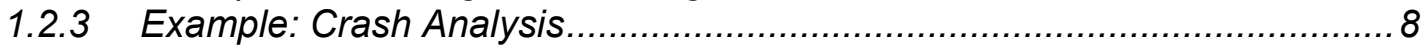

1.3 EMPLOYER AND SPECIAL GENERATOR TRAVEL SURVEYS $\ldots \ldots \ldots \ldots \ldots \ldots \ldots \ldots \ldots \ldots \ldots$

1.3.1 Example: Central Business District Congestion ...................................... 9

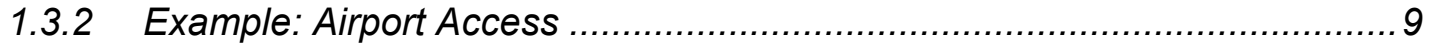

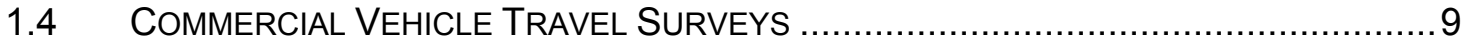

1.4.1 Example: Commercial Vehicle Travel Demand Modeling.......................... 10

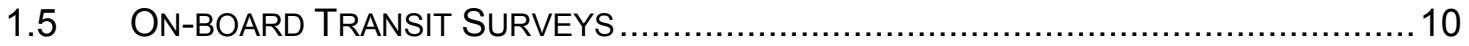

1.5.1 Example: Redesigning Transit Routes ............................................. 10

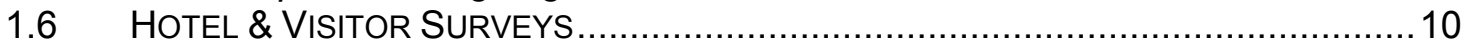

1.6.1 Example: Tourism District............................................................ 11

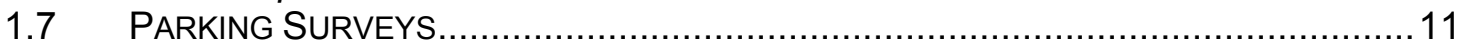

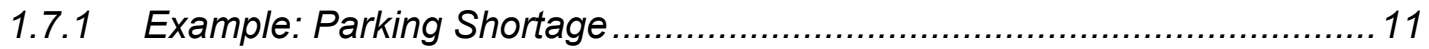

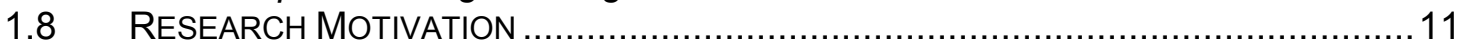

CHAPTER 2 - DESCRIPTION OF DATA COLLECTION TECHNIQUES \& METHODS 13

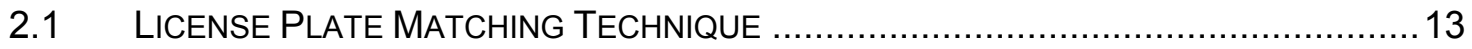

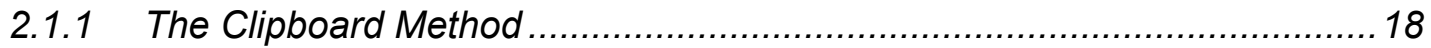

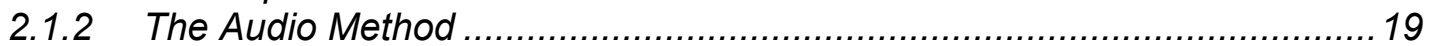

2.1.3 The Laptop Method ...................................................................... 20

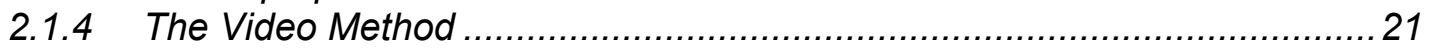

2.1.5 The Photography Method ........................................................... 23

2.1.6 Summary of Methods for License Plate Matching Technique ....................23

2.2 OTHER (NON-LICENSE PLATE) MATCHING TECHNIQUES ...................................24

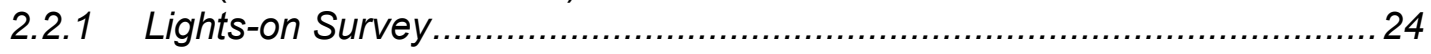

2.2.2 Automatic Vehicle Identification at Toll Stations..................................... 26

2.2.3 Video Imaging........................................................................ 27

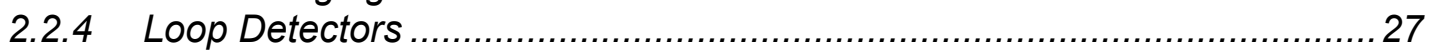

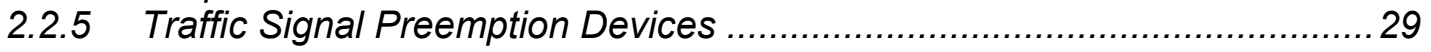

2.3 LICENSE PLATE FOLLOW-UP SURVEY TECHNIQUE...................................... 29

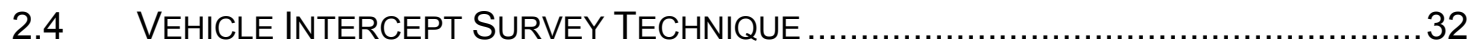

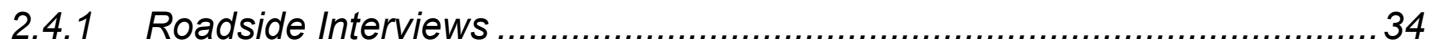

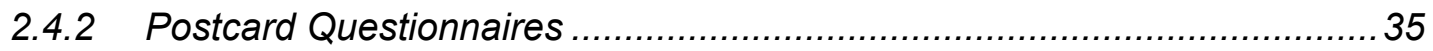

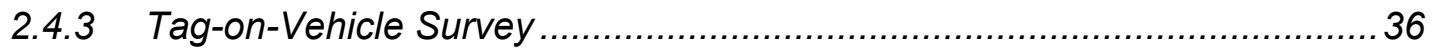

2.4.4 Summary of Methods for the Vehicle Intercept Survey Technique............. 37 


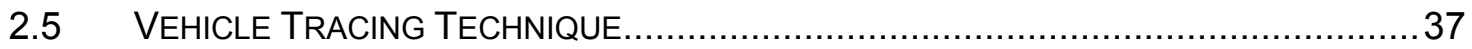

2.5.1 Global Positioning System (GPS) Tracing.............................................. 38

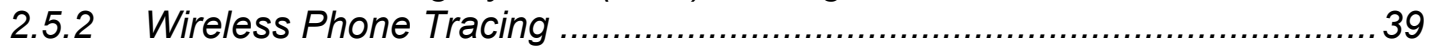

2.5.3 Summary of Methods for the Vehicle Tracing Technique...........................41

2.6 MATHEMATICAL OD ESTIMATION TECHNIQUES ..........................................42

2.7 SUMMARY OF ALL OD TECHNIQUES .................................................... 42

CHAPTER 3 - STATE DOT SURVEYS ON OD TECHNIQUES AND METHODS ..........44

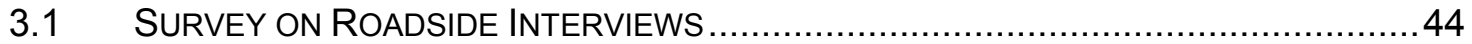

3.2 SURVEY ON ALL OD TECHNIQUES AND METHODS ….................................... 45

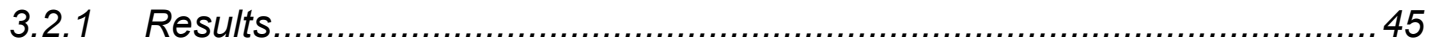

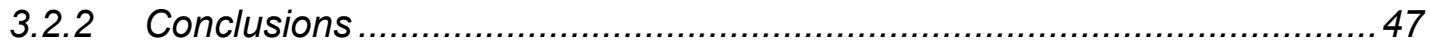

CHAPTER 4 - LEGAL ISSUES OF VEHICLE INTERCEPT AND LICENSE PLATE FOLLOW-UP SURVEYS IN INDIANA........................................................... 48

CHAPTER 5 - EVALUATION OF EQUIPMENT FOR LICENSE PLATE DATA

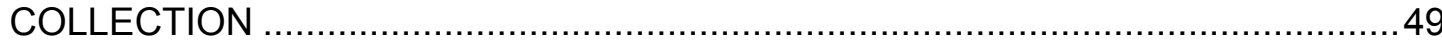

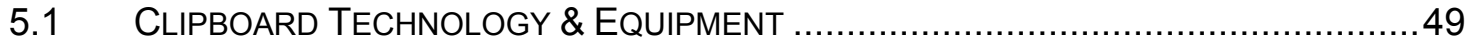

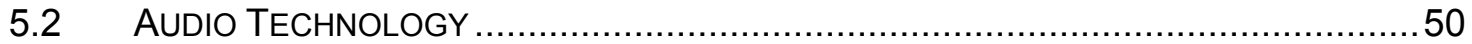

5.2.1 Analog Cassette Audio Recorders ...................................................... 50

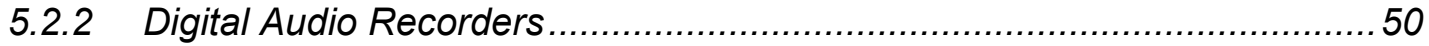

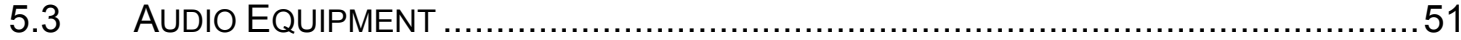

5.3.1 Sony Micro-cassette Audio Recorder ..................................................51

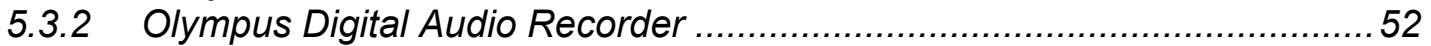

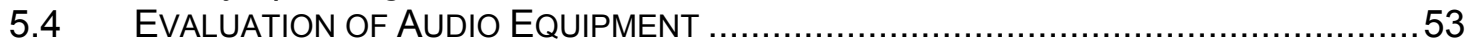

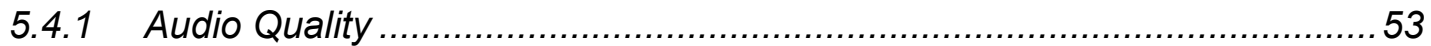

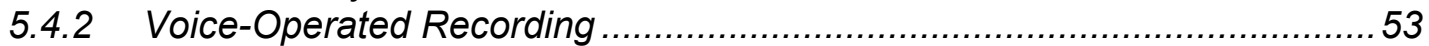

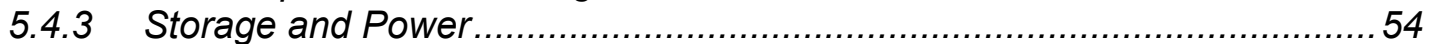

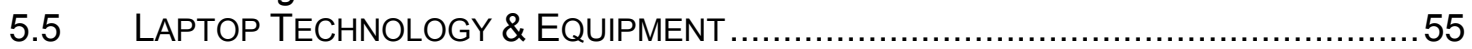

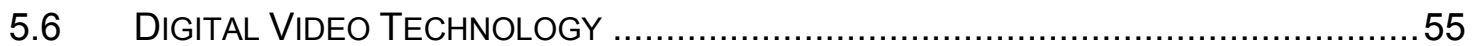

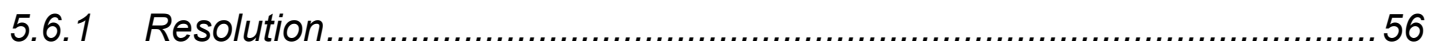

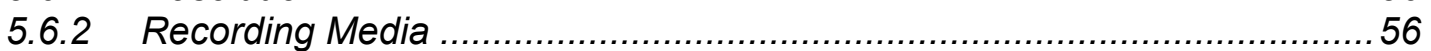

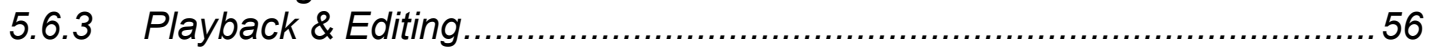

5.6.4 Charge-Couple Device (CCD) ..................................................57

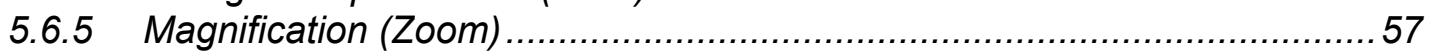

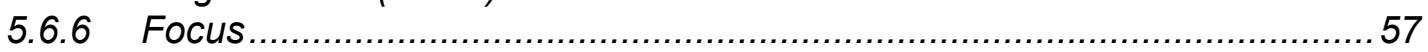

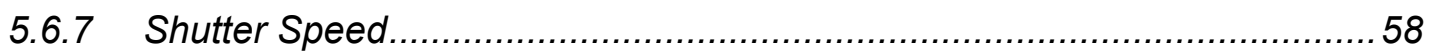

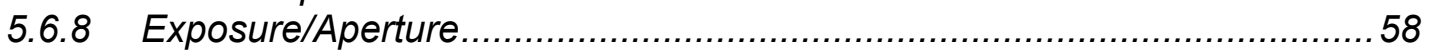

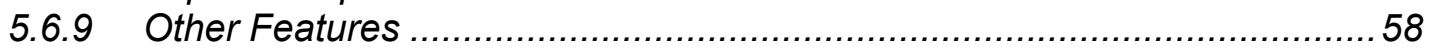

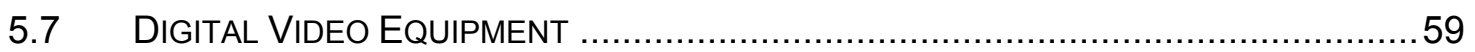

5.8 EVALUATION OF DIGITAL VIDEO EQUIPMENT ................................................6

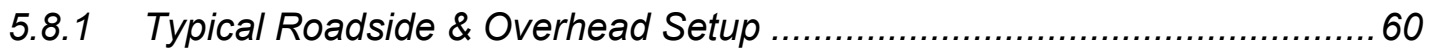

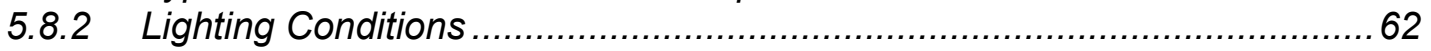

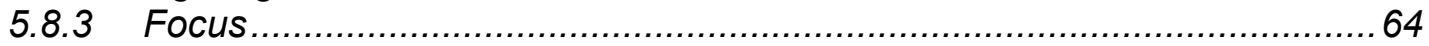

5.8.4 Obstruction \& Minimum Shooting Angles ............................................6

5.8.5 Magnification and Angle-of-View Variations by Camcorder Model..............68

5.8.6 Magnification ............................................................................ 72

5.8.7 Optimal Shutter Speed …............................................................... 73 


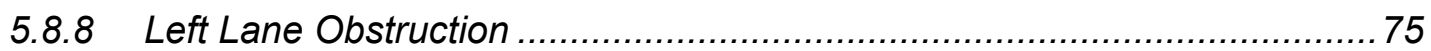

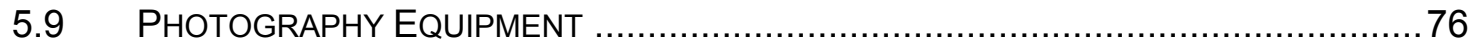

CHAPTER 6 - DETERMINING LICENSE PLATE DATA COLLECTION METHOD .......78

6.1 LICENSE PLATE DATA COLLECTION RULES...............................................78

6.1.1 License Plate Matching Technique................................................... 78

6.1.2 License Plate Follow-Up Survey Technique ............................................. 79

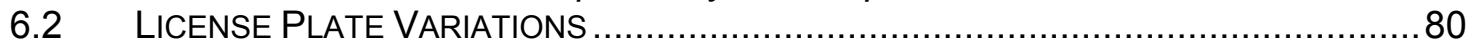

6.2.1 Indiana Passenger Vehicle License Plates .............................................8 80

6.2.2 Indiana Commercial \& Government Vehicle License Plates ......................82

6.2.3 Out-of-State License Plates.............................................................. 83

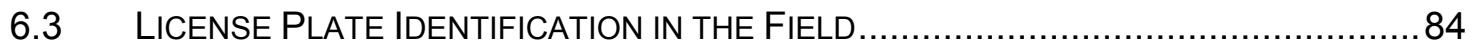

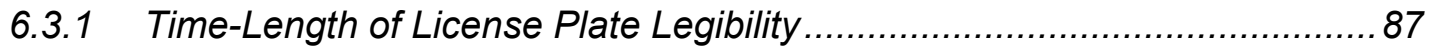

6.3.2 License Plate Identification Time …................................................... 90

6.3.3 Probability of String Identification ....................................................... 90

6.3.4 Maximum Vehicle Speed for Manual License Plate Data Collection ........... 92

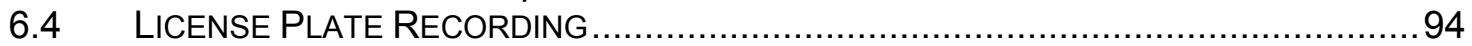

6.4.1 License Plate Recording Time .........................................................94

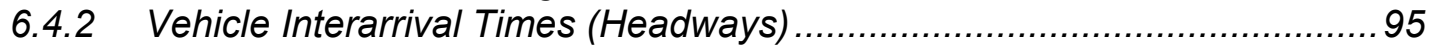

6.4.3 Maximum Flow Rate for Manual Methods ………...................................95

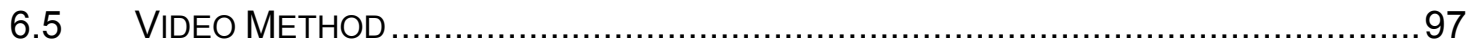

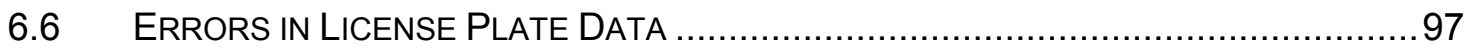

6.6.1 Field and Transcription Errors ............................................................ 97

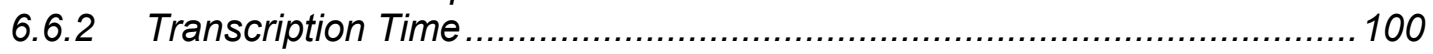

CHAPTER 7 - ACCURACY OF OD STUDY TECHNIQUES .....................................102

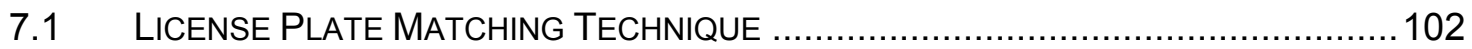

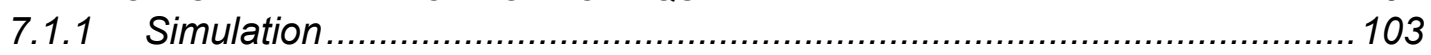

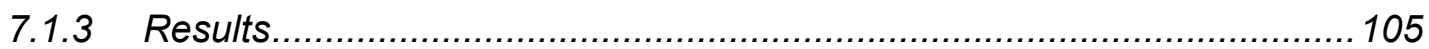

7.2 VEHICLE INTERCEPT AND LICENSE PLATE FOLLOW-UP SURVEY TECHNIQUES ...112

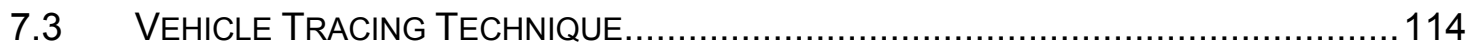

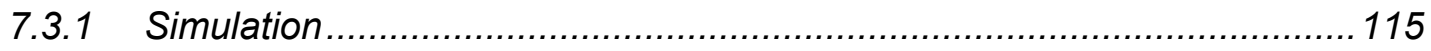

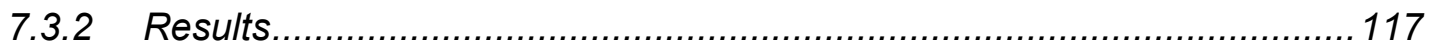

CHAPTER 8 - GUIDELINES FOR SELECTING AN OD TECHNIQUE AND METHOD 120

8.1 SUMMARY OF FINDINGS FOR LICENSE PLATE DATA COLLECTION ....................120

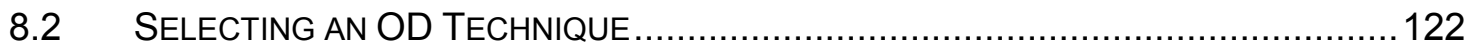

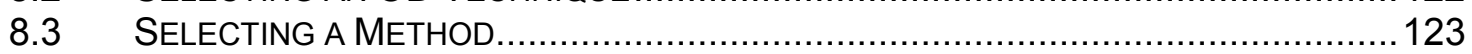

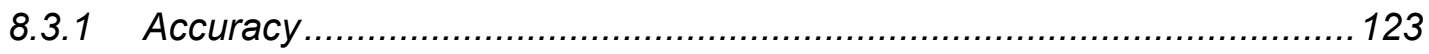

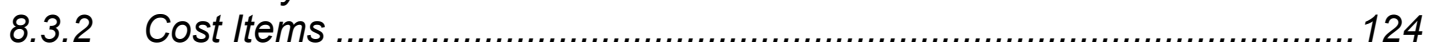

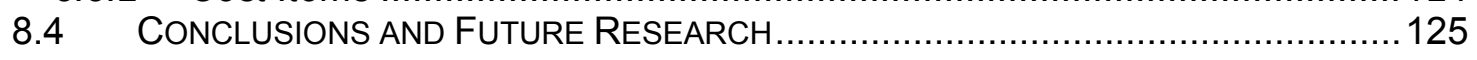

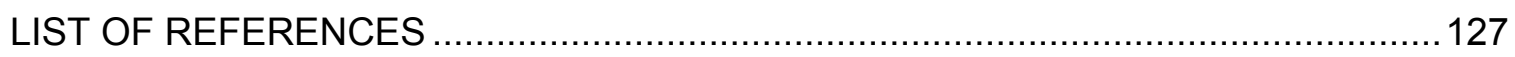

APPENDIX A SURVEY OF STATE DOTS ………..........................................130

APPENDIX B GUIDELINES FOR CONDUCTING LICENSE PLATE SURVEYS ......130 


\section{LIST OF TABLES}

Table

Table 1: Summary of Characteristics for License Plate Data Collection Methods ..........24

Table 2: Summary of Characteristics for Vehicle Intercept Survey Methods .................37

Table 3: Summary of Characteristics for Vehicle Tracing Methods ...............................42

Table 4: Summary \& Comparison of Roadside Station OD Study Techniques ..............43

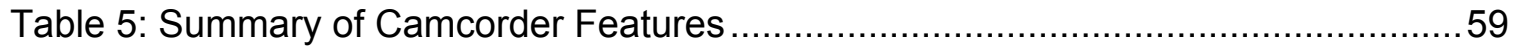

Table 6: Predicted vs. Actual Angles-of-View ..................................................... 71

Table 7: Perpendicular Vehicle Speed (mph) ......................................................... 74

Table 8: Theoretical Time-Length of License Plate Legibility (2.75" Characters)............88

Table 9: Theoretical Time-Length of License Plate Legibility (1.25" Characters)............89

Table 10: Observed Time-Length of License Plate Legibility (1.25" Characters)............89

Table 11: Identification Time (s) by Vehicle Speed ...............................................90

Table 12: Vehicle Speed (mph) above which Video Method is Recommended..............93

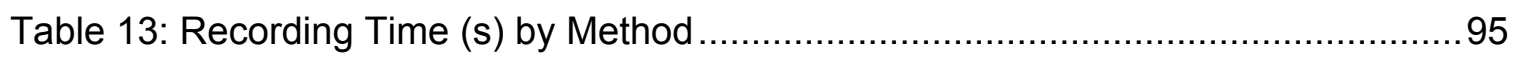

Table 14: Maximum Flow Rate (vphpl) using Manual Recording Methods ....................96

Table 15: Field \& Transcription Errors by Method.................................................98

Table 16: Transcription Time (sec) by Method ..................................................... 101

Table 17: Standard Deviation of Percent Error in Trip Estimations ............................112

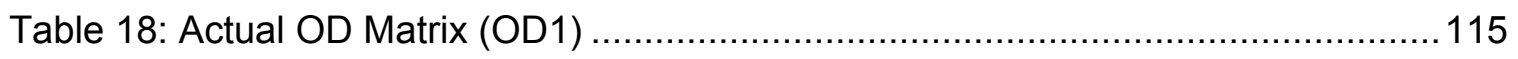

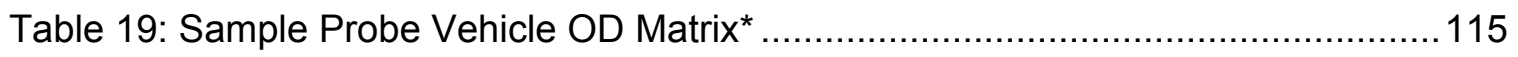

Table 20: Estimated OD Matrix (Expanded Probe Vehicle Matrix) .............................116

Table 21: 4x4 1600-trip Matrix (OD2) without Uniformly-Distributed Cells...................117

Table 22: 4x4 1600-trip Matrix (OD3) without Uniformly-Distributed Cells or Zeros .....117

Table 23: 4x4 16,000-trip Matrix (OD4) with Uniformly-Distributed Cells....................117

Table 24: 8x8 1600-trip Matrix (OD5) with Uniformly-Distributed Cells .......................118

Table 25: General Cost Items by OD Study Technique ...........................................125 


\section{LIST OF FIGURES}

$\begin{array}{ll}\text { Figure Page } & \text { Page }\end{array}$

Figure 1: Traffic Analysis Zones and Types of Trips ............................................

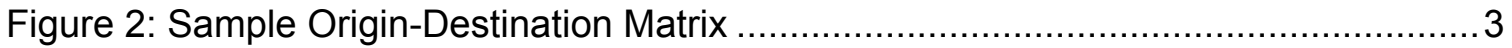

Figure 3: Bypass Analysis for Small Cities \& Towns ............................................ 4

Figure 4: Corridor Origin-Destination Analysis ....................................................... 4

Figure 5: Types of Trips obtained from a Household Travel Survey ............................. 6

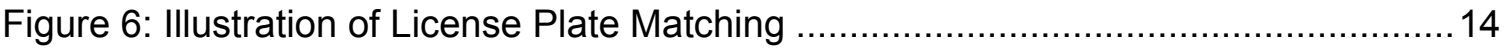

Figure 7: Types of Trips obtained from the License Plate Matching Technique ............15

Figure 8: Proportion of spurious matches for various block sizes \& permutations ..........17

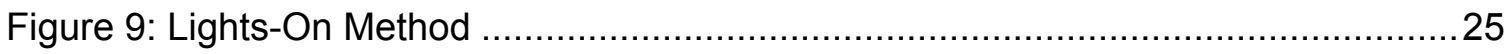

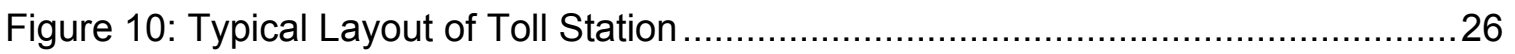

Figure 11: Vehicle Signatures for Various Vehicle Types ...................................... 28

Figure 12: Types of Trips from License Plate Follow-Up Survey Technique .................31

Figure 13: Typical Layout of a Vehicle Intercept Station ......................................... 33

Figure 14: Handset-Based Location Determination using GPS ................................ 40

Figure 15: Network-Based Location Determination using TDOA ..............................40

Figure 16: Plan View of Typical Camcorder Roadside Setup ...................................60

Figure 17: Elevation View of Typical Camcorder Overhead Setup .............................61

Figure 18: Lighting Conditions Encountered on the Roadside ..................................63

Figure 19: Following-Vehicle Obstruction from the Roadside Perspective ....................66

Figure 20: Following-Vehicle Obstruction from the Overhead Perspective....................66

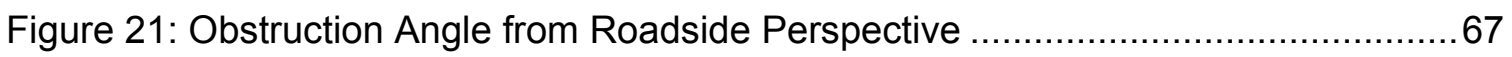

Figure 22: Obstruction Angle from Overhead Perspective ....................................... 67

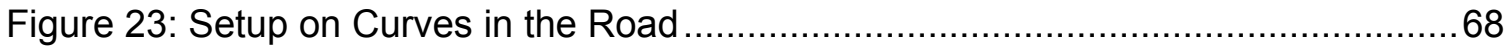

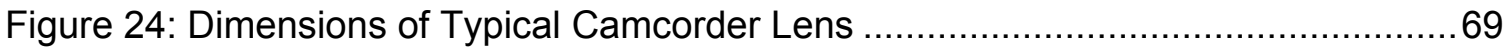

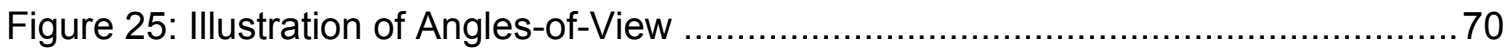

Figure 26: Relationship between Focal Length \& Angle-of-View by CCD Size...............71

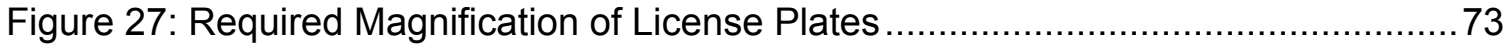

Figure 28: Vehicle Speed Perpendicular to the Camcorder Line-of-Sight.....................74

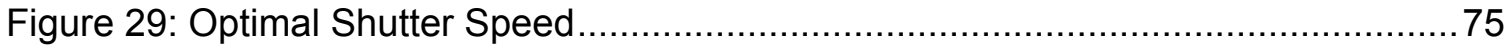

Figure 30: Obstruction of License Plates in Left Lane ........................................... 76 
Figure 31: Left Lane License Plate Obstruction Factor ......................................... 76

Figure 32: Indiana Passenger Vehicle Standard License Plates ..............................81

Figure 33: Indiana Passenger Vehicle Special Recognition License Plates ..................81

Figure 34: Indiana Commercial \& Government Vehicle License Plates......................... 82

Figure 35: Out-of-State Standard Passenger Vehicle License Plates...........................83

Figure 36: Out-of-State Commercial Vehicle License Plates .................................... 84

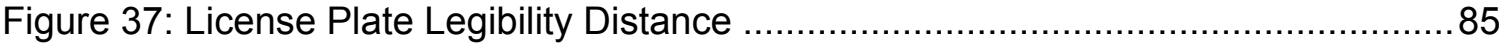

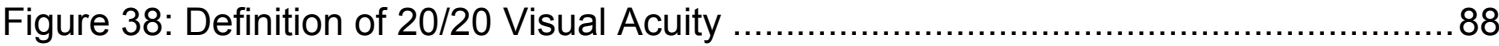

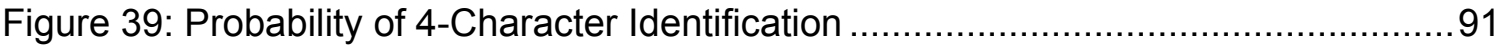

Figure 40: Probability of Full String Identification .................................................. 92

Figure 41: Capture Rates by Vehicle Flow for each Method.......................................96

Figure 42: Error by Proportion for Traffic Volume of 100 Vehicles................................ 105

Figure 43: Error by Proportion for Traffic Volume of 1,000 Vehicles ........................... 106

Figure 44: Error by Proportion for Traffic Volume of 5,000 Vehicles ........................... 106

Figure 45: Error by Proportion for Traffic Volume of 10,000 Vehicles..........................107

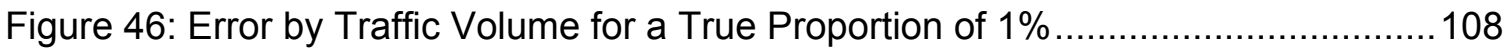

Figure 47: Error by Traffic Volume for a True Proportion of 5\% ............................... 108

Figure 48: Error by Traffic Volume for a True Proportion of $25 \%$................................ 109

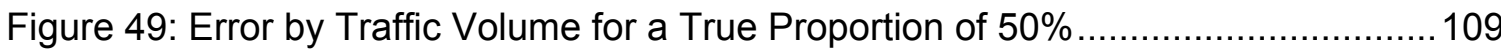

Figure 50: Error by Capture Rate Product for Traffic Volume of 100 Vehicles.............. 110

Figure 51: Error by Capture Rate Product for Traffic Volume of 1,000 Vehicles........... 110

Figure 52: Error by Capture Rate Product for Traffic Volume of 5,000 Vehicles...........111

Figure 53: Error by Capture Rate Product for Traffic Volume of 10,000 Vehicles.........111

Figure 54: PRMSE of Estimated OD Matrices based on OD1 (Table 18)...................116

Figure 55: Mean PRMSE for Various OD Matrices ............................................. 118 


\section{CHAPTER 1 - INTRODUCTION}

Origin-Destination (OD) studies are an important tool for transportation professionals. OD studies are conducted to understand the pattern of the movement of persons and goods in a particular area of interest during a particular period of time (Wang, 1997).

OD studies are typically conducted in order to collect data as a basis for travel demand modeling. At the core of travel demand modeling is the OD matrix, which is essentially the trip distribution step of the four-step modeling process. Once travel demand models are created and calibrated for an area, they can be used to perform a variety of tasks. These tasks include analysis of travel characteristics (such as travel time, delay, and pollution), the impact of modification or closure (due to an incident or road work) of existing routes, and the design and evaluation of the effectiveness of new routes on the existing transportation network. They can also be used to make long range travel forecasts to identify potential future problems in the transportation network and evaluate alternative solutions. Credible travel demand models are necessary for good transportation planning and programming.

The OD matrix is an $n \times n$ matrix, where $n$ is the number of Traffic Analysis Zones (TAZs) in a study area plus the number of entry/exit nodes (external stations) that lie at the cordon line (or boundary) of the study area. The size and number of TAZs depends upon the population, employment, and land use in the area.

Figure 1 illustrates an area with 12 TAZs and four entry/exit nodes. There are five arrows drawn on the figure, which represent the types of trips that are required for a complete OD matrix. 


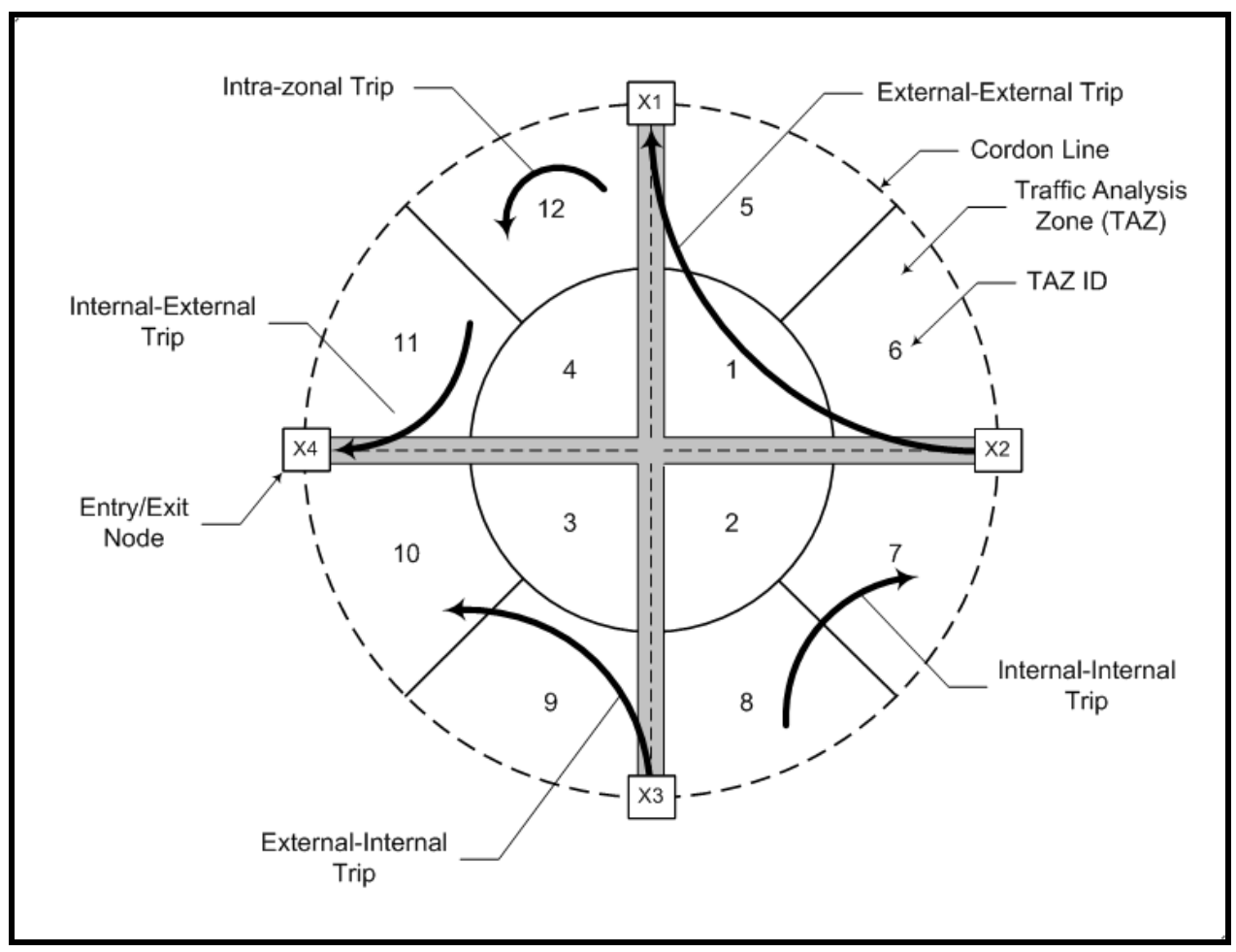

Figure 1: Traffic Analysis Zones and Types of Trips

An internal-internal $(\mathrm{I}-\mathrm{I})$ trip is a trip that has its origin and destination inside the study area. Usually, these trips originate in one TAZ and are destined for another. However, a special type of I-I trip, the intra-zonal trip, is one that has its origin and destination within the same TAZ. An internal-external (I-E) trip is a trip that originates inside the study area, travels through an exit node on the cordon line, and has a destination outside the study area. On the other hand, an external-internal (E-I) trip is one that originates outside the study area, travels through an entry node on the cordon line, and is destined for some TAZ inside the study area. Finally, an external-external $(E-E)$ trip is a trip that has both its origin and destination outside the study area, but passes through the study area via two entry and exit nodes.

In order to obtain a complete OD matrix, the number of trips from each TAZ (and entry/exit node) to all other TAZs (and entry/exit nodes) must be determined. Therefore, the OD matrix for Figure 1 is a $16 \times 16$ matrix (12 TAZs plus 4 entry/exit nodes). In reality, however, OD matrices are much larger, because metropolitan areas may contain hundreds of TAZs. 
Figure 2 illustrates the OD matrix for the area shown in Figure 1. To illustrate the location of the types of trips described above, the cells are shaded and labeled. In reality, each cell would contain a number that represents the number of trips from the origin zone to the destination zone. The sum of each row represents the total number of trips that originate in each zone, and the sum of the column represents the number of trips that have destinations in each zone.

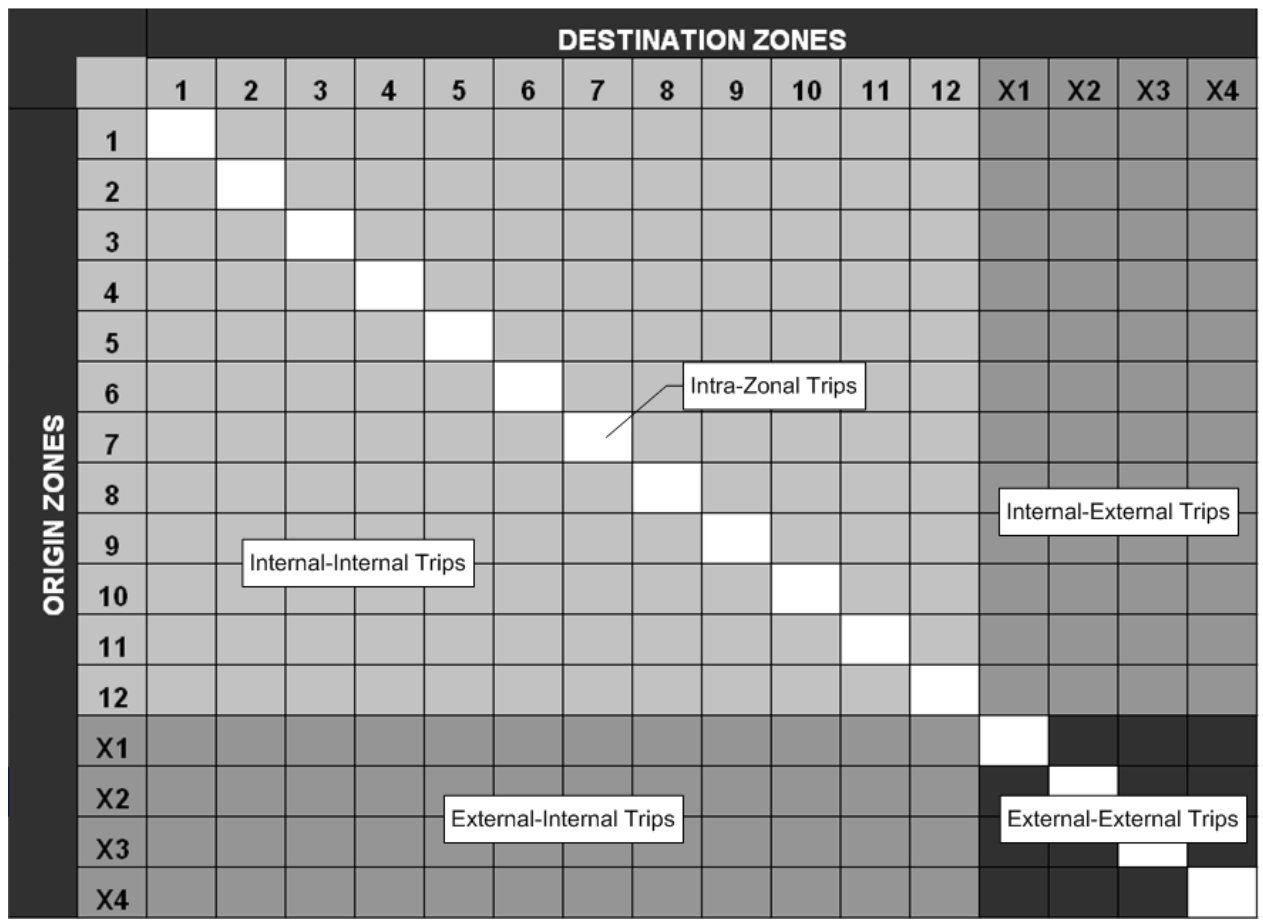

Figure 2: Sample Origin-Destination Matrix

While complex travel demand models are typically developed for large cities, metropolitan planning organizations (MPOs), and state departments of transportation (DOTs), smaller cities and towns without a complete travel demand model sometimes also require OD studies in order to evaluate alternative solutions to transportation problems. These travel demand models can be used to evaluate bypass alignment alternatives, traffic signal coordination and timing scenarios, and corridor safety, among others.

Figure 3 illustrates a bypass analysis for a small city or town. The objective in this situation is to determine the number of E-E trips. The principles are the same as those for a larger study area; however, less attention is given to I-I trips because the 
entire study area is considered to be one or a few TAZs. E-I (and I-E) trips are determined by subtracting the traffic volumes on the link at the entry/exit node from the number of E-E trips determined at that location. This process is conducted at all entry/exit nodes. In the figure, the thickness of the arrow represents the relative traffic flow from only one entry node to all other exit nodes (E-E trips) and the city itself (E-I trips).

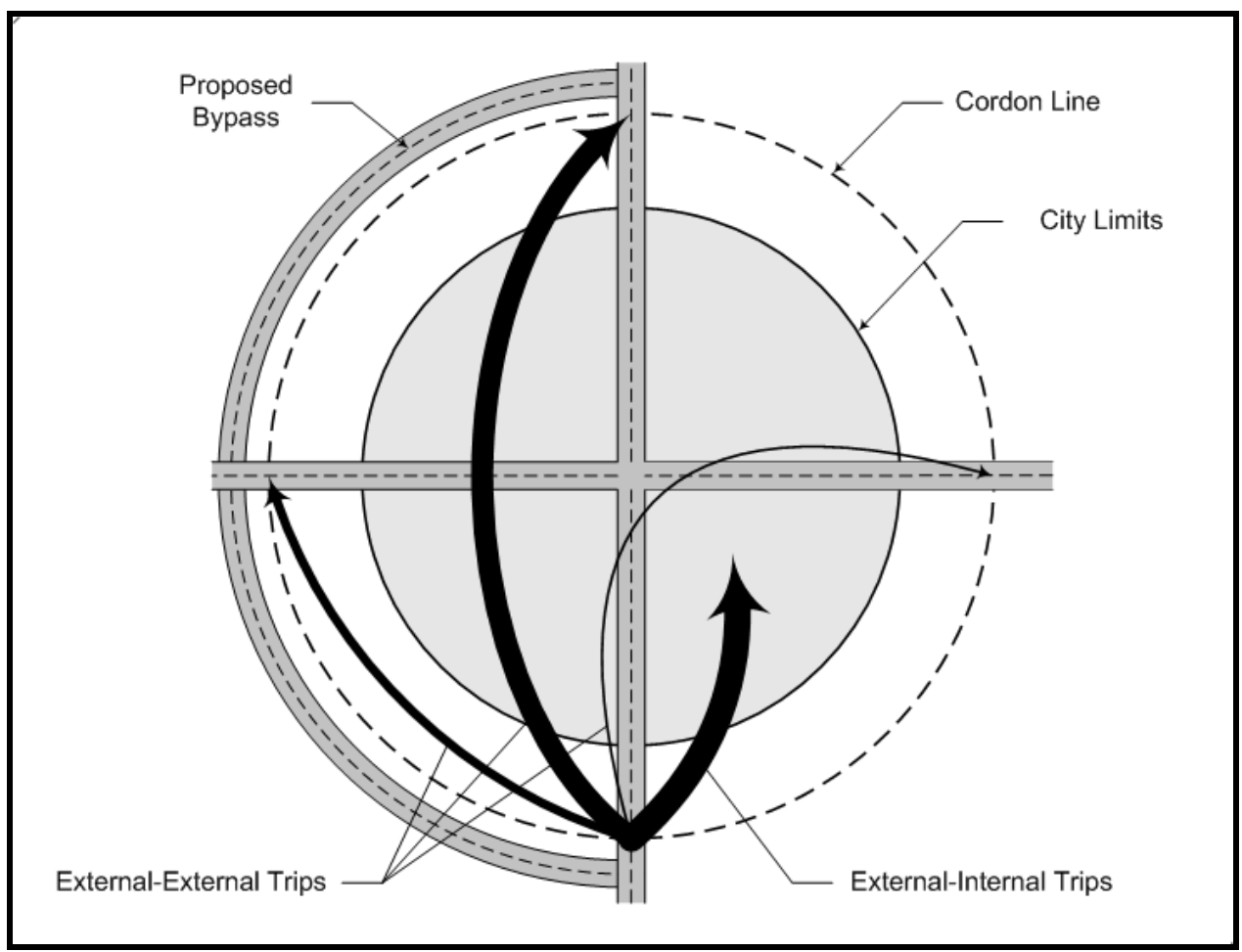

Figure 3: Bypass Analysis for Small Cities \& Towns

Figure 4 graphically illustrates how an OD study might be applied along a corridor for signal timing coordination or safety analysis. Again, the thickness of the arrow represents the relative traffic flow.

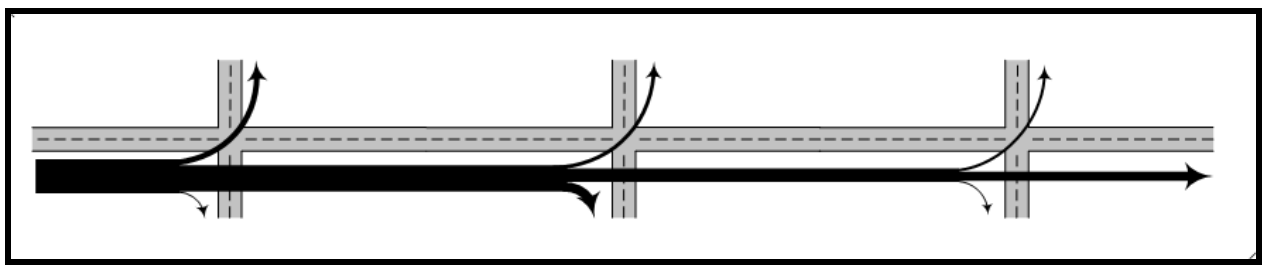

Figure 4: Corridor Origin-Destination Analysis 
Other, more specialized OD studies can also be used to analyze interchange design, special traffic generators such as airports and other large tourist attractions, and parking adequacy. Typically, an OD study can be categorized into one of seven types. The following is a description of each type along with an example where each is commonly applied.

\subsection{Household Travel Surveys (Travel Diaries)}

In this survey, a sample of households within the study area (with varying levels of income, household members, and available vehicles) are selected and recruited for participation. Members of participating households record their household information and travel activity in a travel diary or through a recall interview for a certain time period, generally 24 or 48 hours. The information recorded includes the start time, travel time, trip length, origin, destination, travel mode, trip purpose, and vehicle occupancy of each trip. This information is aggregated with other similar households to establish average trip rates and trip lengths for that type of household. This data is expanded to all households in the study area. By combining this information with trip production and attraction analysis in the first step of the travel demand modeling process, an origindestination matrix can be defined. This type of survey is typically conducted by MPOs as an update to an existing travel demand model, or by the US Department of Transportation Bureau of Transportation Statistics as part of the National Household Travel Survey (NHTS).

Besides administering the survey to a new subset of households for each survey, a panel of households may be used. In a panel sample, the travel survey is given to the same households over time, thus revealing changes in travel behavior as household characteristics (such as the ages of household members) change.

The household travel survey reveals general travel patterns from a sample of the population for all roadways in a particular study area. It does not provide detailed information on any particular roadway within the study area. Figure 5 illustrates the types of trips that are obtained as a result of conducting a household travel survey. 


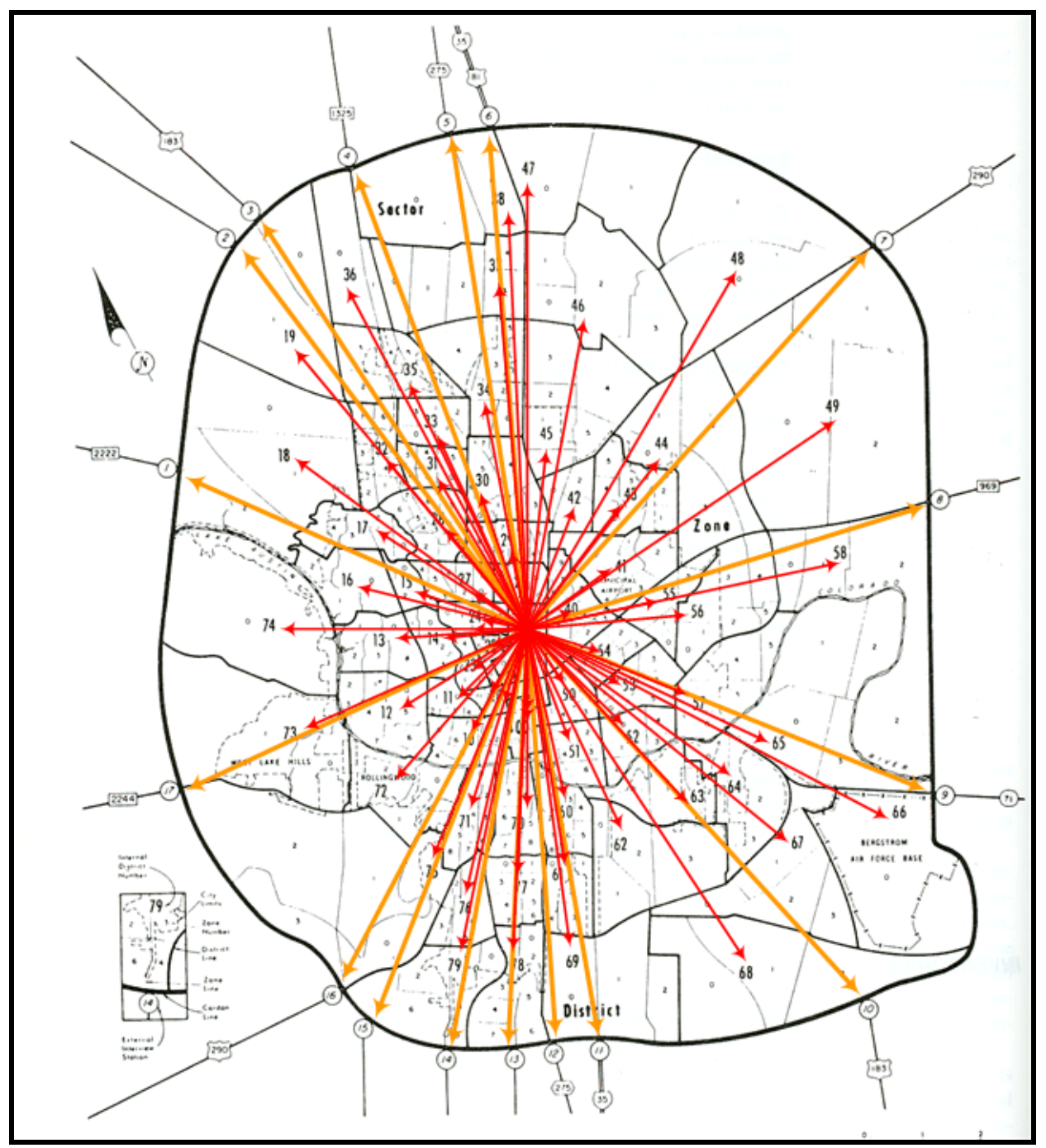

Figure 5: Types of Trips obtained from a Household Travel Survey Map Source: Box \& Oppenlander as shown in the Manual of Transportation Engineering Studies, 1994

The arrows in Figure 5 represent the traffic flows from one TAZ to all other TAZs. The dark-shaded arrows represent inter-zonal I-I trips, while the lighter-shaded arrows represent I-E trips. E-E trips can not be obtained from a household travel survey.

The following examples are some common applications where household travel surveys are generally used.

\subsubsection{Example: Building a Travel Demand Model}

A small metropolitan area is one of the fastest growing areas in the state, and it is expected that it will continue to grow for the foreseeable future. Many citizens are 
upset that road construction is not keeping up with the increased demand on the roadways, and are demanding better short-term and long-term planning strategies. The new MPO in charge of the area's comprehensive plan wants to develop a travel demand model to represent the existing transportation network and forecast future growth so that road construction can be adequately planned and designed in advance of the demand. To do this, a household travel survey will be administered to a sample of all households, and certain households will be recruited to complete a 24-hour travel diary.

\subsubsection{Example: Updating a Travel Demand Model}

A major update of an urban area's travel demand model is not going well. The model's link loadings do not match the observed counts on the corresponding links at all. The mismatches are especially bad on the high-volume links. After ruling out other causes, the modeler suspects the trip table used in the trip distribution step. The table was an update of an origin-destination matrix that was based on a household survey conducted 25 years ago. The update was based on a borrowed equation for the average trip lengths (by trip purpose) in unspecified cities of similar population, but the modeler suspects that the highways that pass through his city cause the borrowed equation to produce erroneous results.

\subsection{Roadside Station Surveys}

In this type of OD study, drivers are directly interviewed or vehicles are monitored (typically via license plates) at a set of roadside stations to determine their travel characteristics through the study area. This survey method is used to determine the number of E-E trips on each road segment at the entry/exit node. Depending on the technique used in this study, I-E and E-I trips may or may not be determined. This will be discussed further in Chapter 3.

Like the household travel survey, this type of study has many uses. The roadside station survey is often used to supplement the household travel survey in creating and validating travel demand models by collecting data at important internal cordons and screen-lines. It can also be used to collect data in a particular corridor for sub-area and small area models. Unlike the data from household travel surveys, data from roadside station surveys are typically not transferable to other applications. The following examples describe some common applications of the roadside station survey. 


\subsubsection{Example: Bypass Feasibility Study}

A small city lies at the intersection of two state highways. In recent years, truck traffic through the small city seems to have increased greatly. The city's residents suspect that most of the heavy trucks do not have origins or destination in the city; they are just passing through. In doing so, the trucks are destroying the pavement on the city's two main streets and causing bad traffic tie-ups at the city's principal intersection. If it can be proved that most truck traffic is through traffic, a strong case for building a bypass around the city can be made.

\subsubsection{Example: Traffic Signal Re-timing}

A major US highway passes through a medium-sized city, which also serves as a major arterial in the city's street network. The traffic signals along that highway corridor are timed as if most of the traffic is through traffic. Some people think that much of the traffic on that highway has at least one trip end in the city - at least during peak periods. If that is true, the city's travel demand model must be revised to reflect those trip patterns and the corridor's traffic signals should be retimed to accommodate the turns on and off the highway.

\subsubsection{Example: Crash Analysis}

A particular section of urban interstate highway in a major city has an unusually high crash rate. Many of these crashes seem to be sideswipe and rear-end collisions. The highway section is located between two entry/exit ramps that are rather close to each other. Some observers think that weaving is a problem. If the pattern with which vehicles enter and exit the interstate at these two locations can be established, the expensive redesign of those two entry/exit points can be evaluated.

\subsection{Employer and Special Generator Travel Surveys}

This type of survey is used to collect information on establishments with trip attractions. These establishments may be businesses or other unique special generators such as commercial airports, arenas and convention centers, amusement parks, or other tourist attractions. This type of survey is often conducted because the establishments have high attraction rates that have unique characteristics and cover a 
large geographic area. If the survey is being conducted for one or a few establishments, the survey can be conducted as an intercept survey as people enter and exit a building or site similar to an roadside station survey. Otherwise, to gather information on a large number of establishments, the survey may be distributed to a sample of the population at each establishment, similar to a household travel survey. The following are some examples where employer or special generator travel surveys may be used.

\subsubsection{Example: Central Business District Congestion}

During the afternoon rush-hour in the CBD of a large city, traffic congestion at the freeway on-ramps create long delays and is a safety concern of drivers and pedestrians. In an effort to reduce congestion during this time, surveys were sent out to major employers in the CBD to determine if any alternatives (such as alternate routes, staggered business hours, or transit subsidies) would reduce delays and increase safety.

\subsubsection{Example: Airport Access}

Annual passenger enplanements at an airport in a large city have grown steadily the past several years. Traffic congestion at the airport's loading and unloading area at the terminal is creating long delays and is a safety hazard for pedestrians. The city and airport are considering constructing a new light-rail line connecting the central business district about three miles away to the inside of the airport terminal. It is important to determine the percentage of airport patrons coming and going from the CBD, and just as important, where within the CBD. Therefore, a survey will be given to patrons, and for those with trip ends in the CBD, a stated preference survey will be administered to evaluate use of the light rail line.

\subsection{Commercial Vehicle Travel Surveys}

This survey type is used to obtain OD data for trucks and other commercial vehicles. This information can be utilized to determine trip rates, commodity flows, and air quality modeling within an area. The following is one example where a commercial vehicle travel survey may be used. 


\subsubsection{Example: Commercial Vehicle Travel Demand Modeling}

In recent years, a metropolitan planning organization has been updating its travel demand model. Because most household travel surveys do a poor job of defining commercial trips, and traffic counts cannot distinguish between a personal trip and commercial trip for light-duty vehicles, a new category of trip is being created to be included in the updated travel demand model which will better define those trips. Therefore, data will need to be collected on the travel patterns of vehicles such as package delivery vehicles, postal vehicles, couriers, equipment repair and service technicians, craftsmen (carpenters, plumbers, etc.), government workers, and taxis, all of which may use personal or light-duty vehicles for commercial purposes.

\subsection{On-board Transit Surveys}

This type of survey is used by modelers or transit agencies. Information is typically collected on characteristics of the users and their travel patterns. The transit origin-destination matrix can be used in travel demand models or by transit agencies to analyze service changes and improve the performance of the transit system. The following is an example of one application of an on-board transit survey.

\subsubsection{Example: Redesigning Transit Routes}

Over the course of the last several years, the ridership on a city's bus system has declined. At the same time, much of the new commercial development has occurred on the city's edge. In an effort to expand to the new development, the bus company has extended the existing routes into the new areas, which has caused more stops and delay for all of the riders. Officials want to know if breaking up these routes and adding express routes will slow or stop the ridership decline. As part of this analysis, a survey of passengers on-board the buses will be conducted.

\subsection{Hotel \& Visitor Surveys}

This type of survey seeks to collect data on travel conducted by visitors that stay in hotels and motels and who are out of reach of the household survey. Typically, these groups of people have much different travel characteristics than the residents of the same city. This type of OD study could be considered a type of special generator survey 
described in Section 1.3. The following is an example of one application of a hotel/visitor travel survey.

\subsubsection{Example: Tourism District}

A particular tourist district of a city contains a mixture of land uses, including entertainment, shopping, and recreational establishments, hotels, and an amusement park. The streets in and around this district are highly congested. Many of the patrons are out-of-town visitors who are unfamiliar with the area. The city and property owners of the district are interested in building a transit system to link the district's top attractions in an effort to reduce the congestion on the streets. A survey of hotel guests will be conducted to determine the daily travel patterns of these visitors on weekdays and weekends.

\subsection{Parking Surveys}

Parking surveys are sometimes used to obtain detailed parking information for travel demand models to evaluate parking supply, costs, and subsidies on travel decisions. This can be done as people enter and exit parking facilities or through a questionnaire placed on the windshield of the vehicle that is voluntarily completed and returned. The following is an example of one application of a parking survey.

\subsubsection{Example: Parking Shortage}

The central business district of a city is experiencing a lack of available public parking. The city currently owns several public garages, but is considering constructing several more. In order to determine the locations of future garages, a parking survey will be administered to the users of existing garages to determine the type and location of the activity for which they are being used.

\subsection{Research Motivation}

As indicated earlier, OD studies can take on many forms. However, all studies require a certain amount of effort for data collection, and the quality of data can have a major influence on the results of the study. In addition, many studies are restricted by time and cost. Therefore, it is imperative that the amount and quality of data collected is adequate for each and every study. It is generally not easily known, however, what data 
collection method should be used for a particular type of OD study because of cost restraints, data quality, and the reliability and accuracy of the results after postprocessing.

While quite a lot of information has been published on how to best conduct household travel surveys and travel diaries, especially in the Travel Survey Manual and other documents published by the Travel Model Improvement Program (TMIP), less information has been published on the best methods for conducting roadside station surveys in regard to the data collection involved with each of those methods. The purpose of this study is to evaluate both the conventional and experimental techniques for conducting roadside station origin-destination studies through literature review, surveys, field testing, and computer simulation, and subsequently develop guidelines for conducting them. These guidelines, however, apply only to roadside station OD studies, which are just one of the seven types of OD studies described in Chapter 1.

The Indiana Department of Transportation (INDOT), in conjunction with the Purdue University Department of Civil Engineering, seeks to develop general guidelines that will serve as a starting point for planning and conducting future INDOT roadside station OD studies. These guidelines will be designed for transportation professionals, DOTs, consultants, and others who desire to conduct a Roadside Station OriginDestination Study. 


\section{CHAPTER 2 - DESCRIPTION OF DATA COLLECTION TECHNIQUES \& METHODS}

Data for origin-destination studies can be collected in many ways, particularly for the roadside station survey. However, it is not always easy to determine which method is the best for obtaining accurate data while minimizing cost with the resources available. Each method has a unique set of characteristics with respect to planning, data collection, and data analysis. The techniques and methods described in greater detail in this chapter refer only to Roadside Station Origin-Destination Studies. To clarify, the word "technique" refers to the procedure in which data is being collected, and "method" refers to the means by which the procedure is carried out.

\subsection{License Plate Matching Technique}

In this technique, partial license plate strings are recorded by an observer at a roadside station. In addition, the time of day is recorded from a timepiece that has been synchronized with all other stations. Traffic volume counts are usually conducted simultaneously (with manual traffic counters) in order to obtain a "capture rate" for expansion of the data to the population. Vehicle classification may also be observed and recorded for each license plate. The record of all license plates recorded from one roadside station is compared to records from other stations within the study area. $A$ match is noted if a license plate is seen at two stations within a certain time parameter, that is, upper and lower bounds of reasonable travel time between the two stations. Figure 5 illustrates this process. 


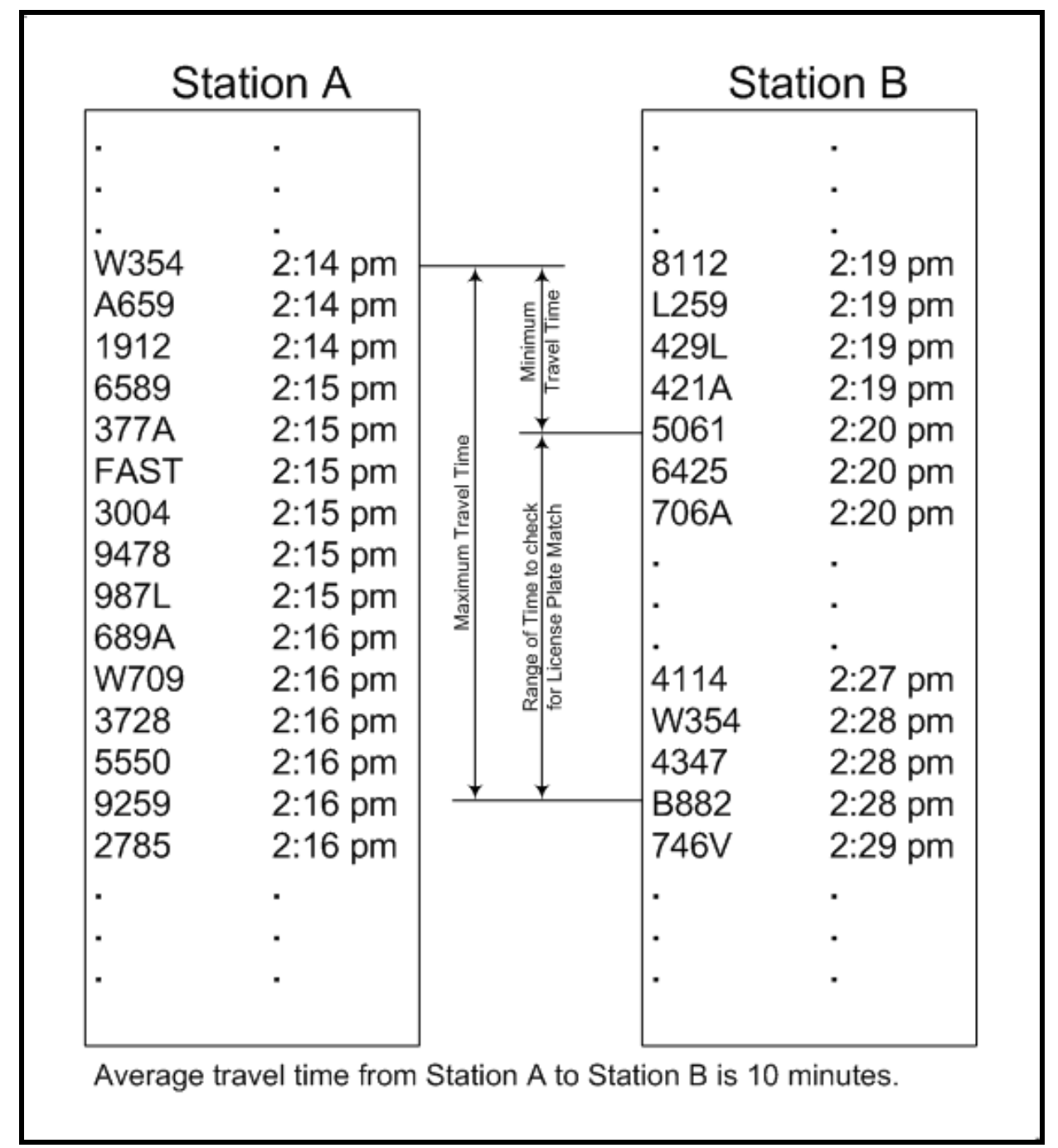

Figure 6: Illustration of License Plate Matching Source: Adapted from Slavik (1986)

Once each license plate has been evaluated for a match with all other stations, the data is then expanded to determine the number of all vehicles at a particular station that pass by any of the other stations, which are considered E-E or through trips. Figure 7 illustrates the types of trips that are obtained as a result of a cordon station license plate match. The lighter-shaded arrows represent E-E trips from one entry node to all other exit nodes on the cordon line. The single dark-shaded arrow represents all E-I trips from the entry node to all TAZs. While the license plate matching technique can determine the number of E-I trips, it is unknown how these E-I trips are distributed to each TAZ. This technique is most appropriate when conducting OD studies on small 
cities and towns where internal trips (I-E or E-I) do not have to be assigned to a particular TAZ (origin or destination) inside the cordon line.

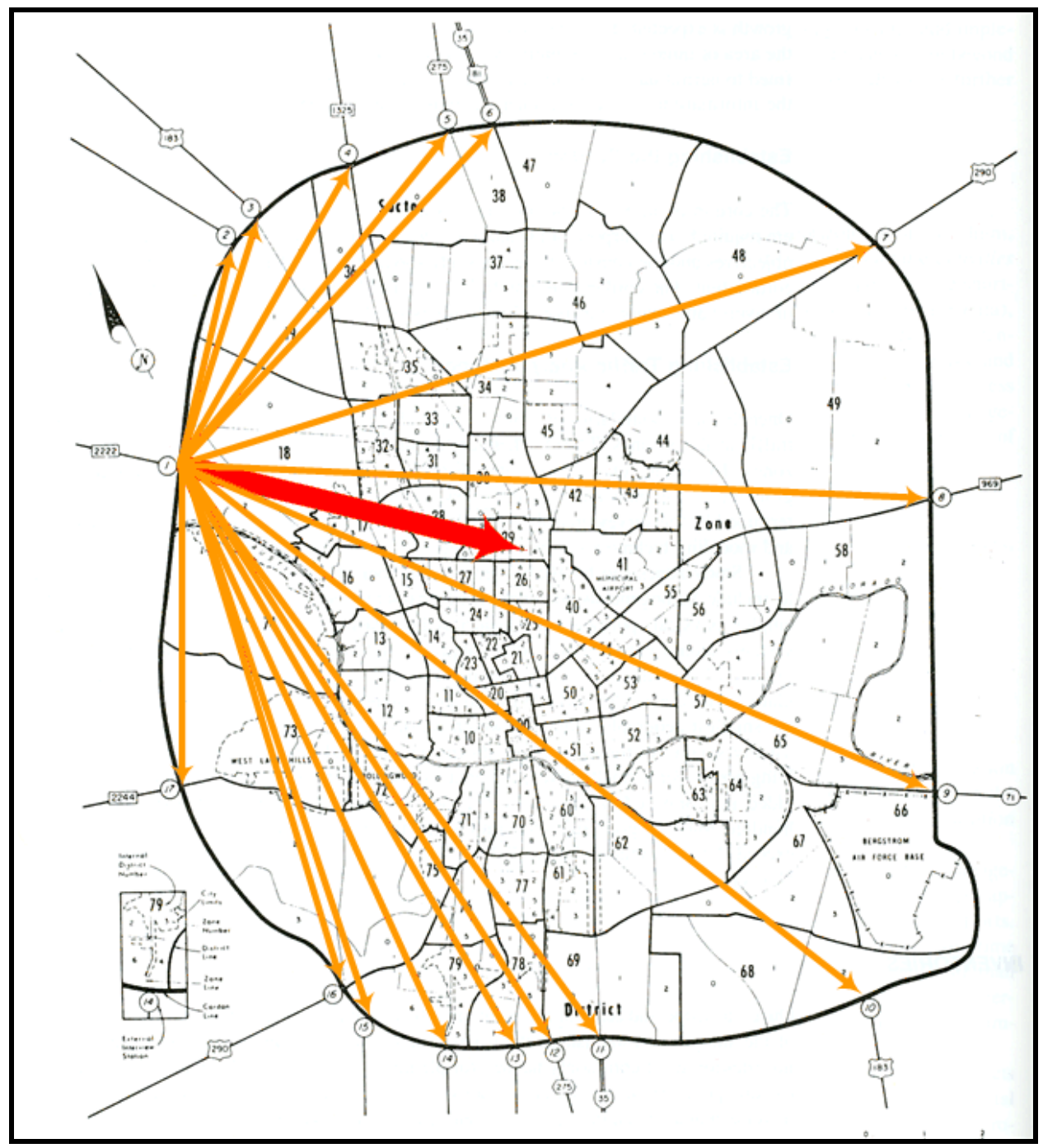

Figure 7: Types of Trips obtained from the License Plate Matching Technique

The license plate matching technique has several advantages. The only data that is recorded is the license plate, time, and if necessary, vehicle classification. Because there are no driver surveys and vehicles are only monitored from the roadside, this technique is unobtrusive to the drivers and safer for the observers. The data reduction process is simpler than that of a questionnaire, but may or may not be as timeconsuming. In addition, the amount of data that can be collected for a particular road is only limited to the means by which it is being collected. In other words, a fairly large 
ratio of the vehicles on the roadway can be sampled relative to that of one observer with other techniques. According to Turner (1996), this data can provide an estimate of travel times (if there are enough matches and precise time stamps) over the course of the study period. Also, the technique can be used on most types of roads (Turner et al., 1998).

However, some disadvantages exist. The weather can pose a problem depending upon the method chosen to record license plates, and fatigue can be a problem for observers if the data collection period is too long or too intense. Also, errors can be introduced in several processes during the data collection and reduction periods of identifying, recording, and transcribing each license plate. In addition, large amounts of data can be lost due to equipment failure (such as loss of power in audio recorders, laptops, or camcorders in the field during the study), which would require redoing parts of the study, which is both costly and time-consuming. Locations for the roadside stations are important, given that vehicles traveling in platoons (particularly near signals) or low speeds may block the view of other vehicles' license plates, especially if camcorders or cameras are used to record them. Similarly, vehicles traveling freely at high speeds may pass too quickly to be recorded. Furthermore, some vehicles may have license plates that are missing, damaged, dirty, covered, or blocked. For large surveys, adequately training and mobilizing the observers can be a challenge (Martin, 1993).

There has also been some concern about spurious matches, which are produced when two partial license plate entries that actually belong to two different vehicles are matched (Slavik, 1986). However, Slavik determined that the effect of spurious matches could be virtually eliminated by updating the time at least every 5 minutes and/or increasing the number of characters (or permutations) recorded for each entry. The number of permutations $N$ depends on how many characters are recorded. For three numbers, there are 1,000 permutations $\left(10^{3}\right) .10,000$ permutations are achieved by recording four numbers $\left(10^{4}\right)$ or three letters $\left(22^{3}\right)$, assuming that 22 of the 26 letters of the alphabet are used on license plates. Figure 8 illustrates this. 


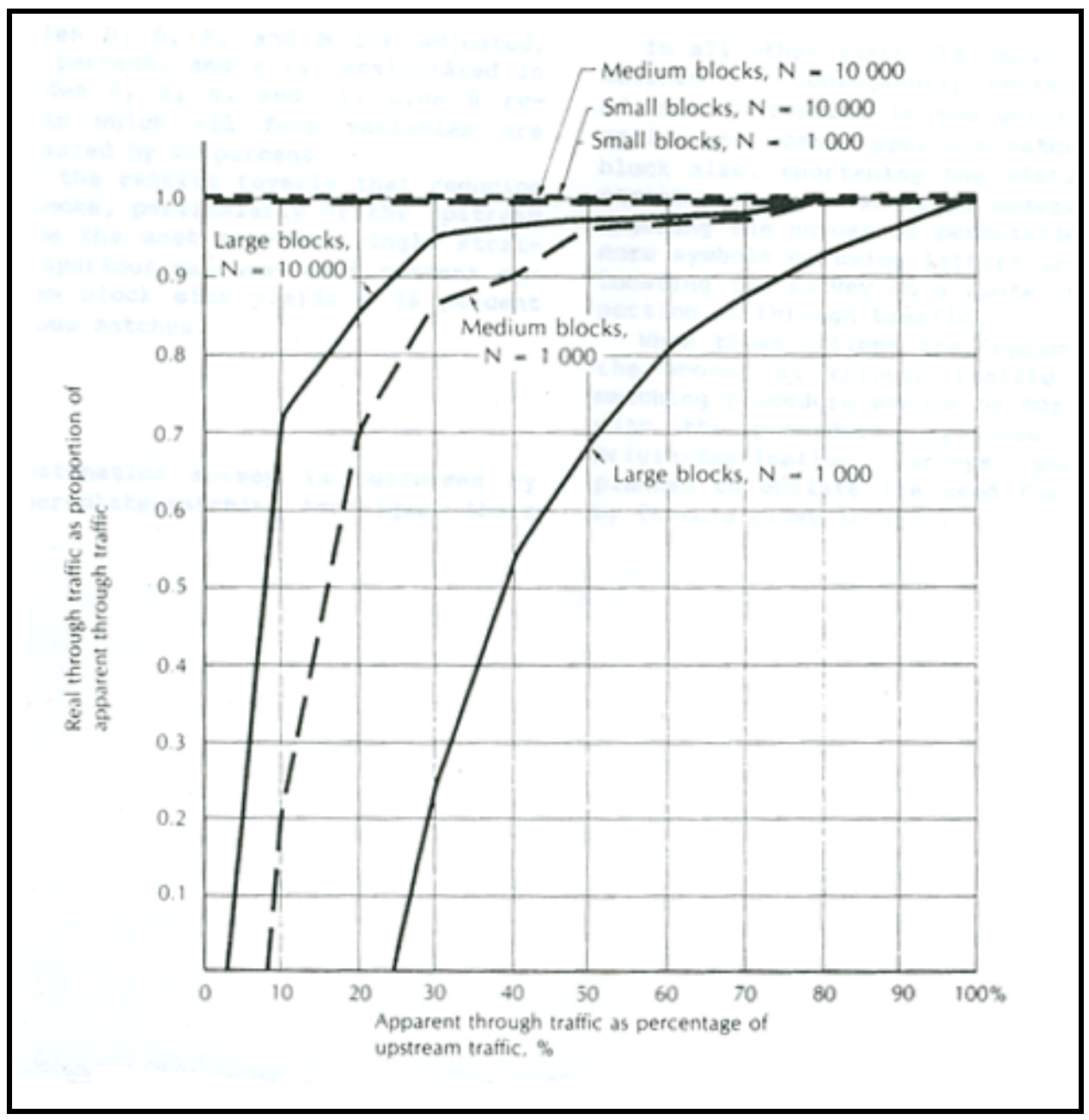

Figure 8: Proportion of spurious matches for various block sizes \& permutations Source: Slavik (1986)

In Figure 8, the size of the block refers to the precision with which the time stamps are recorded for each license plate entry. Small blocks refer to precision of less than 5 minutes; medium blocks are between 5 and 15 minutes; and large blocks are greater than 15 minutes.

The effect of spurious matches is only an issue when large blocks are used for any number of permutations, or for medium blocks with 1,000 permutations. In most situations, it is not difficult to obtain small blocks. It is important, however, that timepieces be synchronized between stations. 


\subsubsection{The Clipboard Method}

This method requires observers at each station to manually record with a paper and pencil the partial license plate number of as many passing vehicles as possible on a form. During intervals between vehicles, the time stamp should also be recorded (ideally every minute but not greater than five minutes). According to Martin (1993), observers can record approximately 170 full license plates per hour or 800 partial license plates per hour (three or four digits).

Vehicle classification may also be recorded, but simultaneous traffic counts should be conducted in order to obtain an accurate capture rate (which is used later for data expansion). These records must be manually transcribed into software for matching after the data collection period is over.

The clipboard method is the most conventional method of collecting the data. It does not require the purchasing and supplying of any expensive equipment to the observers. In addition, little time has to be spent on training staff.

There are disadvantages in using a clipboard, however. First of all, the license plate entries recorded on the clipboard may be illegible to varying degrees depending upon the quality of penmanship of each individual observer. Even one incorrectly recorded letter or digit in the license plate string will eliminate a potential match. Emphasis must be placed on the legibility of the penmanship of the observer. The accuracy of each recorded license plate is more important than the quantity of license plates recorded. If possible, the transcription of license plates should be done by the observer. In addition, the time stamp that is recorded along with each license plate record will not be as accurate as other methods (as will be discussed later), so the travel times calculated from the field data may not be reliable in all situations. Weather conditions, such as rain, can make this method difficult. Finally, according to Turner et al. (1998) it takes a long time (approximately 10 hours per hour of data collected) to transcribe and match the license plates.

One variation of the clipboard is the tablet PC. In recent years, tablet PCs have become popular and less expensive. A tablet PC is virtually an electronic clipboard, which stores the information in an electronic file rather than on paper. However, the data recorded in the field will still have to be manually transcribed to be matched. While handwritten character recognition technology exists to a degree in some tablet PCs and 
PDAs, it is likely unsuitable for recognizing quickly-written license plate strings due to the fact that recognition accuracy is extremely important. Therefore, this was not evaluated as part of this study.

\subsubsection{The Audio Method}

In this method, observers speak the partial license plate string of as many passing vehicles as possible into some audio recording device. Like the clipboard method, the time should be updated during intervals in which vehicles are not present. The observer may also collect information about vehicle classification if desired. Traffic counts must also be conducted simultaneously, or the observers can note passing vehicles that were unidentified (by saying "no ID" into the recorder) in order to obtain a capture rate for expansion of OD pairs to the entire population of vehicles on the road. According to Martin (1993), between 1000 and 1200 license plates per hour can be recorded by one observer. These voice records must then be played back after the data collection period and manually transcribed into software for matching with other roadside stations.

The audio method also has several advantages and disadvantages. The very nature of audio recording allows the observer to speak, rather than write or type, the license plate numbers into a recorder. The observer does not have to repeatedly look up and down between the license plate and clipboard/computer screen for each entry. This will likely allow the observer to record license plates at a faster rate than the other methods. Audio recorders are also less susceptible in poor weather conditions than clipboard, laptop, and video methods. Audio recording equipment is fairly inexpensive at this time.

Depending upon the tone, pitch, and speaking rate of the observer's voice, some of the license plate records may be inaudible or indecipherable during the transcription process. In addition, the noise from the traffic in the background may drown out the observer's voice and make the transcription process difficult as well. Mistakes made on the audio tapes cannot be easily erased from the record in the field and may cause confusion during the transcription process. Furthermore, like the clipboard method, time stamps cannot be recorded as accurately as other methods. Transcription generally takes two to three hours per hour of data collected (Turner et al., 1998). It can be difficult to transcribe letters, because $B, C, D, E G, P, T$, and $V, M$ and $N$, and $F$ and $S$ 
sound similar. The phonetic alphabet (alpha, bravo...) works better but requires lots of time and resources to train staff (Martin, 1993). Higher-pitched (women's) voices are typically easier to understand, and it is best for the observers to transcribe their own audio recordings if possible.

Like handwritten character recognition discussed in the previous section, speech recognition software exists that will convert spoken words into digital characters, which has the potential to eliminate the time-consuming process of transcribing the license plate strings from the audio recordings to the software for matching with other stations (Washburn et al., 1997).

The main advantage in using speech recognition software is the time saved transcribing the license plate records manually into software. As stated above, manual audio transcription typically takes two to three hours for every hour of tape (Turner et al., 1998).

Speech recognition software, however, has generally been created for recognizing spoken words, not for recognizing individual letters and numbers of a license plate string on a noisy roadside station. Unfortunately, this may cause the automatic transcription process to have very high error. Evaluation of this technology was not conducted as part of this project.

\subsubsection{The Laptop Method}

In this method, the person at each station is equipped with a laptop computer into which he or she types partial license plate string into the computer. The strings are stored along with an exact time stamp (to the nearest second) assigned by the computer after the entry is completely entered. Like the clipboard and audio methods, traffic volume counts must be conducted to determine the number of missed vehicles. Unlike the other methods (clipboard, audio, video, or photo), the laptop method does not require a subsequent transcription step, because the license plate strings and time stamps are entered directly into the computer in the field. 9004 -character strings can be recorded per hour with this method (Turner et al., 1998).

As with the clipboard method, there are advantages and disadvantages in using a laptop for recording license plate strings. Laptops are advantageous in data collection, because the license plate records will all be legible, although they still may not be keyed in correctly in the field. A time stamp can also be accurately recorded to the nearest 
second for each license plate, allowing precise travel times to be calculated for matched vehicles. Furthermore, transcription is not necessary, resulting in no transcription errors and time saved in post processing the data.

However, as with the clipboard method, license plates will be recorded incorrectly at varying degrees depending on the typing skills of the observer. In addition, it may not be cost effective to provide a laptop for each data collector, especially if there are many stations in the study. In addition, laptop computers cannot be utilized in the rain. Still another problem is providing an adequate amount of power to each laptop for the entire study period, which could be problematic for long study periods.

\subsubsection{The Video Method}

This method requires observers to record the license plates on all passing vehicles with a camcorder. The video tapes are then reviewed in the office where partial license plate numbers, time stamps, and vehicle classification (if desired) are transcribed. Missed vehicles also need to be counted. Vehicles can be missed (seen but have unidentifiable license plates) due to a number of reasons, such as the camcorder's improper field-of-view, poor video quality (because of lighting or focus), blockage by another vehicle, or simply the absence of the license plate on the vehicle.

Video data collection also has many advantages compared to other methods of license plate string data collection. With video, camcorders can be set up on the side of the road to record the license plates of passing vehicles, eliminating the need to manually record the license plates on the roadside, especially during long study periods, high speed, or high traffic flow conditions. The license plates can be transcribed manually in the lab at a rate the recorder can control in order to maximize the number of license plates recorded. Transcription typically takes 10 hours for every hour of analog recording (Turner et al., 1998). There is also a permanent record with video, which can be stored and referred to later if necessary. The time stamp on video can also be accurate to the nearest second. Typically, video is the best method to use on high speed, high flow facilities (Shuldiner, 1996).

There are some disadvantages in using video for data collection. Depending on the study size, a large number of video camcorders may be necessary. This equipment can be expensive and sensitive to weather conditions. It is also not well established how to obtain the most legible license plates due to weather conditions such as sun and 
glare, traffic conditions such as flow rate, speed, and headways, and camera settings such as zoom, shutter speed, exposure, and these settings may have to be changed throughout the study period. Also, video camcorders do not necessarily eliminate manpower, as a person may be required to be at the camcorder site to keep the camera running (battery power and tape replacement) and to prevent theft. In addition, while a lot of license plates can be recorded, this method still requires someone or something to transcribe the license plate strings from the video to a computer. Like the audio method, this process is a long, monotonous, and sometimes frustrating process.

Like the clipboard and audio methods described earlier, technology exists to convert the license plate strings from the video automatically to digital characters. There are a few steps in this process. First, the video has to be filtered so that one frame containing a license plate is from each passing vehicle is saved (the others can be removed). Second, the actual license plate has to be found within the frame. Finally, the digits on the license plate have to be read from the video frame (Gupta et al., 2002).

The obvious and biggest advantage in using character recognition for transcription is the time saving over manual transcription. Even if only the first step of the process is completed (frame filtering), the time to manually transcribe the license plate strings is greatly reduced.

The biggest disadvantage in using automatic transcription is that there may still be some transcription error (due to the capabilities of the license plate reader and poor quality of video). Automatic transcription typically yields fewer license plates than manual transcription, although it can be combined with manual transcription (Shuldiner, 1996), in which case, a human tries to identify license plate characters that the machine cannot. This technology is also relatively new, so most software is proprietary and not readily found on the market. For these reasons, character recognition of video images was not evaluated as part of this project. If used, however, there may be additional constraints during the video recording process on the roadside. License plate transcription systems are not standardized, are sensitive to ambient conditions, and can be costly for small studies (Turner et al., 1998). 


\subsubsection{The Photography Method}

The final license plate matching technique to be discussed is still photography. In this method, still photos are triggered by vehicles and taken of license plates as they pass by the observer. Precise time and date stamps can also be applied to the photos.

In one Japanese application, fixed overhead cameras were able to obtain $90 \%$ capture with $5 \%$ error of recognizable characters on freeways. The cameras can also record color and size of the license plate (Asakura et al., 2000).

The major advantage of still photography is the reduced transcription time and elimination of recording error. Like the video method, most automatic license plate readers search for a video frame that contains a license plate (like a still photo) from which the license plate number can be obtained. Essentially, the photo method eliminates the frame-filtering process of the video method.

Few studies have been documented that use this method, and it is unknown if this method has ever been used for an origin-destination application. However, lessons can be learned from other applications such as systems that monitor red light running at signalized intersections. In that application, several photos from several different camera locations are used to obtain the license plate of red-light running vehicles so citations can be mailed to the vehicle owner. In this situation, however, the setup is usually permanent.

For OD purposes, the cameras would have to be able to take pictures at a high rate (approximately every 2 seconds on average). The cameras would also need a triggering device that would indicate when the license plate is going to be in the field-ofview. In addition, the setup location needs to be flexible for moving from one study location to another.

While this method was not directly evaluated as part of this project, many of the same issues discussed for video data collection apply to this method as well. These issues are discussed in Chapter 6.

\subsubsection{Summary of Methods for License Plate Matching Technique}

Table 1 below lists some of the characteristics of data collection methods for the license plate matching technique. Each column represents one of the five data collection methods described in this chapter. The table entries qualitatively assess these characteristics, so that each method can be compared with the others. 
Table 1: Summary of Characteristics for License Plate Data Collection Methods

\begin{tabular}{|l|c|c|c|c|c|}
\cline { 2 - 7 } \multicolumn{1}{l|}{} & Clip & Aud & Lap & Vid & Photo \\
\hline Equipment costs & low & med & high & high & high \\
\hline Observer training & low & low & low & high & high \\
\hline Setup difficulty & low & med & med & high & high \\
\hline Subject to weather & yes & yes & yes & yes & yes \\
\hline Power required & none & low & high & high & high \\
\hline Require separate traffic counts & yes & yes & yes & no & yes \\
\hline Precise time stamps (travel times) & no & no & yes & yes & yes \\
\hline Maximum traffic speeds & low & low & low & high & high \\
\hline Maximum traffic flows & low & med & low & high & high \\
\hline Recording accuracy & med & med & med & high & high \\
\hline Manual transcription time & med & med & none & high & med \\
\hline Automatic transcription accuracy & med & med & none & med & med \\
\hline Permanent Record of LPs & no & no & no & yes & yes \\
\hline
\end{tabular}

\subsection{Other (Non-License Plate) Matching Techniques}

The techniques described below are similar to the license plate matching technique described above. However, instead of matching the license plates of passing vehicles, each technique tries to match some other characteristic of the vehicle. This technique requires the same number of roadside stations as the license plate matching technique. While each of the methods are described below along with advantages and disadvantages, these methods were not evaluated further as part of this project.

\subsubsection{Lights-on Survey}

In this method, vehicle headlights (instead of license plates) are matched between stations. To do this, the driver is simply asked to turn on his or her headlights for the remainder of that trip, usually via roadside work zone signs or a dynamic message sign. Then, observers at other roadside stations record the percentage of passing vehicles with its headlights on. Figure 9 illustrates this method. 


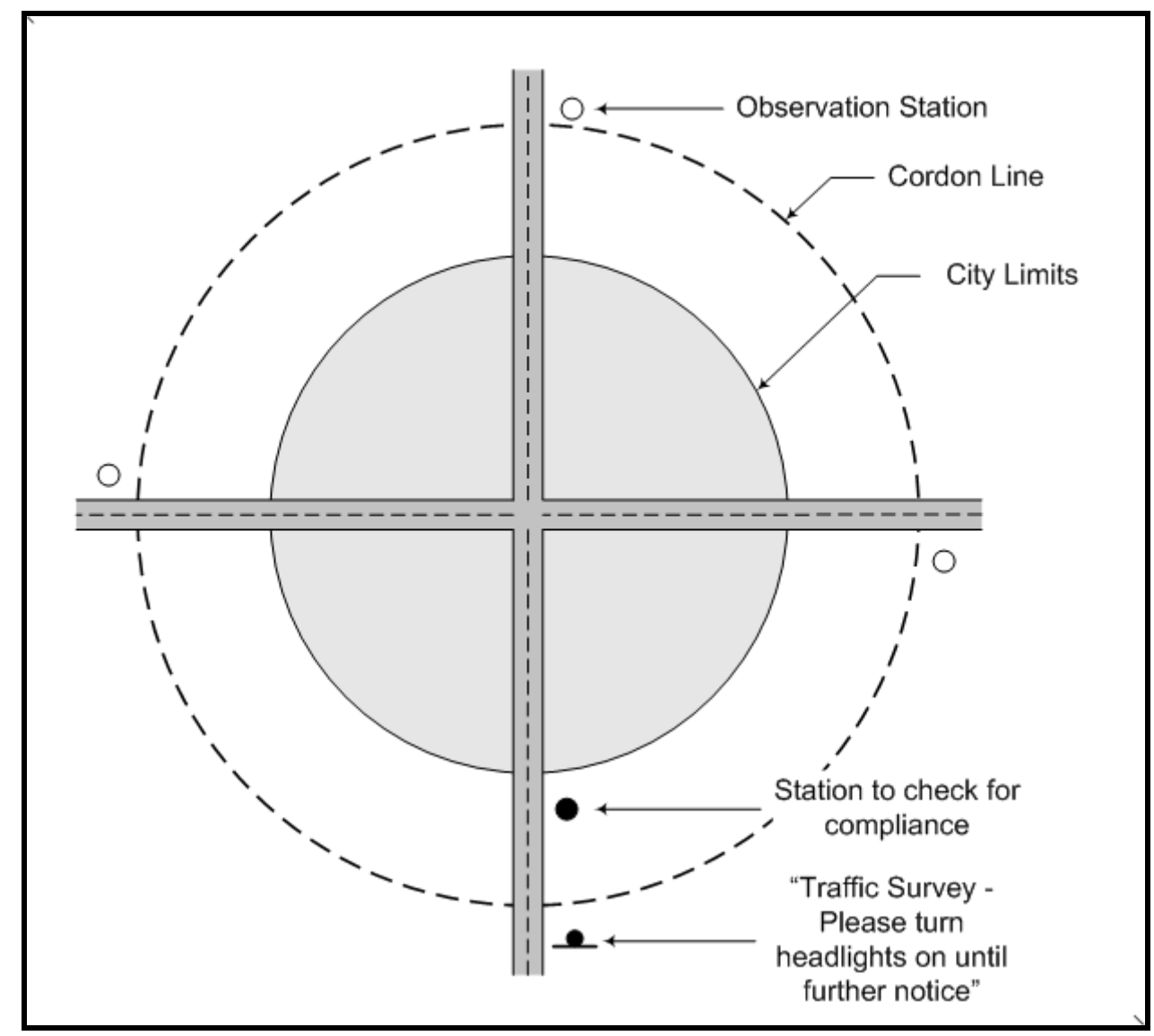

Figure 9: Lights-On Method

A lights-on survey is a simple way to conduct an OD study. However, only information about origin and destination stations can be obtained. Other information, such as travel time and trip purpose, cannot. In addition, observers can generally maintain a safe distance from the roadway.

Today, with the increasing use of daytime headlights on many vehicle models, a lights-on survey could have a high rate of error. It is also unknown how many drivers would comply with the initial request to turn on the vehicle's headlights and subsequently turn them off at a later station. Separate studies to determine these values would be required before or after the study. In addition, only one origin can be studied at a time. Therefore, for a study area that contains multiple roads, each road will have to be studied on separate days. Finally, this technique is limited to daylight hours only. 


\subsubsection{Automatic Vehicle Identification at Toll Stations}

In many large cities across the United States, toll roads are utilized as part of the transportation system. Many of the toll stations located on these roads are equipped with a system that is designed to allow frequent road users to pass quickly through toll stations without stopping in the manual toll lanes. These users typically purchase a toll tag that is mounted on the dashboard or window of the vehicle. This toll tag has a unique identifier that is attached to an account that contains prepaid funds by the owner of the vehicle. When the vehicle passes through the automatic toll lanes at a toll station, a scanner or reader obtains the identification of the vehicle via radio frequency and deducts the toll from the account. Figure 10 illustrates how the system is set up.

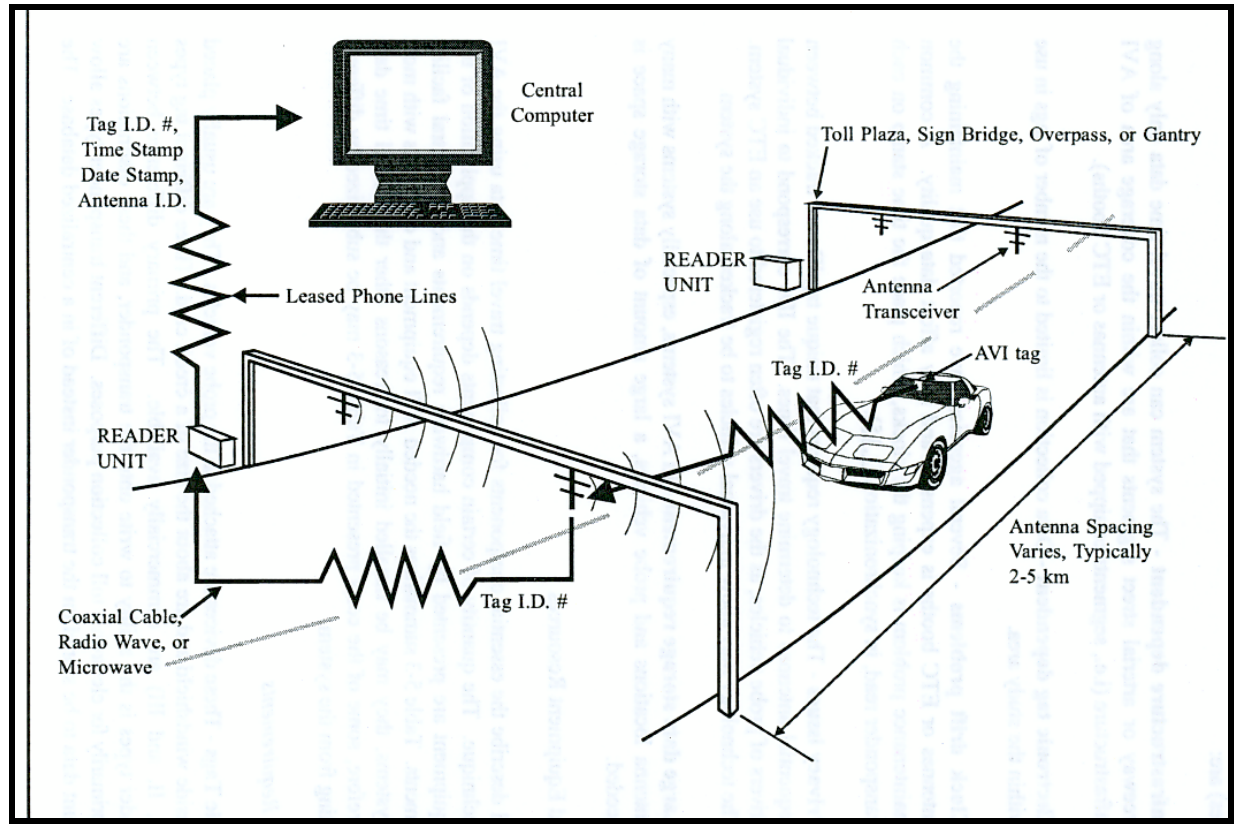

Figure 10: Typical Layout of Toll Station

Source: Turner et al. Travel Time Data Collection Handbook (1998).

This type of setup could be used for OD applications. In this technique, the observer is replaced by the toll tag scanner. Toll tag identifiers, rather than license plates, are recorded and matched between stations. Accurate time stamps could also be obtained for travel times. Toll systems (which often use some sort of radio frequency technology) is very efficient at identifying vehicles. Typically, the recognition rate is close to $100 \%$. 
On the other hand, some people may consider the use of toll tag information to be an invasion of privacy. Furthermore, only a fraction of road users have toll tags, and those users may not be a representative sample of the population of users on the road. For example, toll tag owners typically have a higher income and are more frequent users of the toll system than the average population of vehicles and subsequently will have different travel characteristics. Finally, toll tag reading technology is limited to the roadways on which they are set up, and they cannot simply be moved to conduct a study elsewhere.

\subsubsection{Video Imaging}

This type of system has been tested, although it has been used more extensively for travel time estimation than in OD applications. Video imaging systems work by capturing images of vehicles at different locations and matching them based on vehicle parts such as the hood, roof, trunk, or wheels, as well as overall shape and color. However, most of the testing was done on freeway corridors where the stations were relatively close (Turner, 1996). The expansion of video imaging to an OD application is likely unfeasible at this time due to the large numbers of similar vehicles that would be recorded in a given interval.

The disadvantages seem to outweigh the advantages at this time. For example, it would not be easy to distinguish one black Ford Taurus SE from the next on any given roadway without recording some other unique identifier (such as a license plate number), which is just as easily done using the video license plate matching method discussed above.

\subsubsection{Loop Detectors}

Inductive loop technology is another method that has been studied in the literature and suggested for use in an OD application (Oh et al., 2003). This is done by utilizing a loop detector card to measure inductance, or a "vehicle signature," that is created by the electrical inductance of the loop as a vehicle passes over it. Vehicles of different sizes and number of axles produce unique vehicle signatures that can be matched among multiple loops. Figure 11 illustrates the vehicle signatures for various vehicle types. 


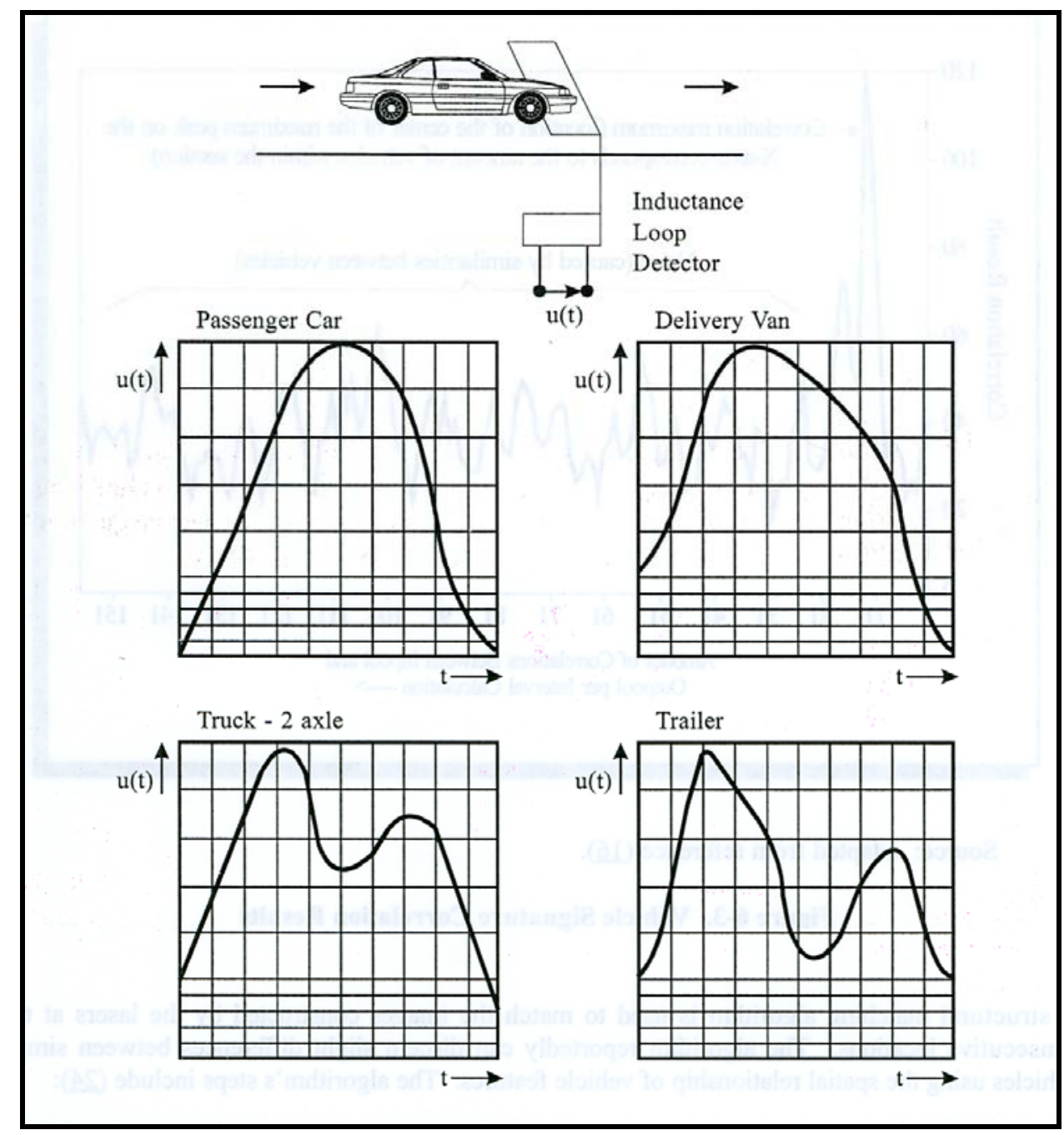

Figure 11: Vehicle Signatures for Various Vehicle Types

Source: Turner et al. Travel Time Data Collection Handbook (1998).

This method may have certain advantages in a signalized network with closelyspaced intersections. In those situations, it is likely that loops already exist on the intersection approaches.

This method, however, faces many of the same problems as video imaging does. For example, the electronic signature left by one Ford Taurus should almost be identical to the next Ford Taurus, which limits the area over which such a method can be applied (for example, beyond an intersection). In addition, inductive loops are expensive to install and immobile once they are installed. Furthermore, most loops probably do not have detector cards capable of measuring (most loops are either activated or inactivated) and storing vehicle signature data. 


\subsubsection{Traffic Signal Preemption Devices}

It has also been suggested in the literature to utilize traffic signal preemption devices (often found in emergency vehicles) in place of license plate observers. This method is very similar to electronic toll tags because, like toll tags, emergency preemptors have a unique identifier associated with them. When a signal picks up an approaching emergency preemptor, it first has to identify it to make sure that it is a legitimate vehicle to adjust the traffic signal.

With this technology, it would be ideal for the receiver to obtain and record the identifier of preemption devices without actually adjusting the traffic signal to unapproved (non-emergency) vehicles. This could then be utilized much like the technology of the toll system.

Signals in many cities already have emergency preemption devices on their main roadways. It is unknown, however, but unlikely that any are capable of actually identifying and storing this type of information.

The major disadvantage is that, in order to conduct an origin destination study on a large scale, preemption devices would have to be distributed to the drivers in the network. This in itself makes this method highly impractical. Most cities would probably be unwilling to use these emergency systems for this type of traffic data collection.

\subsection{License Plate Follow-Up Survey Technique}

This technique uses one of methods described above in order to record license plates at a particular roadside station. A list of license plates is then supplied to the motor vehicles department (DMV) to obtain contact information for the vehicle owner. A survey is sent to the vehicle owner, who is then asked to respond to a survey of questions regarding the specific trip on which their license plate was recorded.

In order to obtain contact information of vehicle owners from the DMV, the full license plate must be recorded. Depending upon the recording method and the requirements of each DMV, the license plates may or may not have to be transcribed into a specific format.

Once the contact information is obtained from the motor vehicles department, a survey of the vehicle owners can be conducted. It is critical that the date, time stamp, location, direction of travel, and other relevant information (such as how their vehicle 
was recorded and contact information obtained) be included in the information provided to the vehicle owner. This survey is usually conducted via a telephone interview or postcard mail-out with response via mail-in, telephone, and/or internet.

License plate follow-up surveys have resulted in both successful and unsuccessful OD studies. They are beneficial in that they are unobtrusive like the license plate matching technique, but detailed information (trip purpose, true origin and destination, etc.) can still be obtained from the actual driver of the vehicle using that specific road. Figure 12 illustrates the types of trips that can be obtained from the license plate follow-up survey technique. Like the license plate matching technique, the lighter-shaded arrows represent the trips from one entry node to all other exit nodes (E-E trips). However, instead of one dark-shaded arrow that aggregates all E-I trips from the external station to the internal TAZs, information provided from the license plate followup survey technique provides information on the distribution of the E-I trips to each of the TAZs inside the cordon line. 


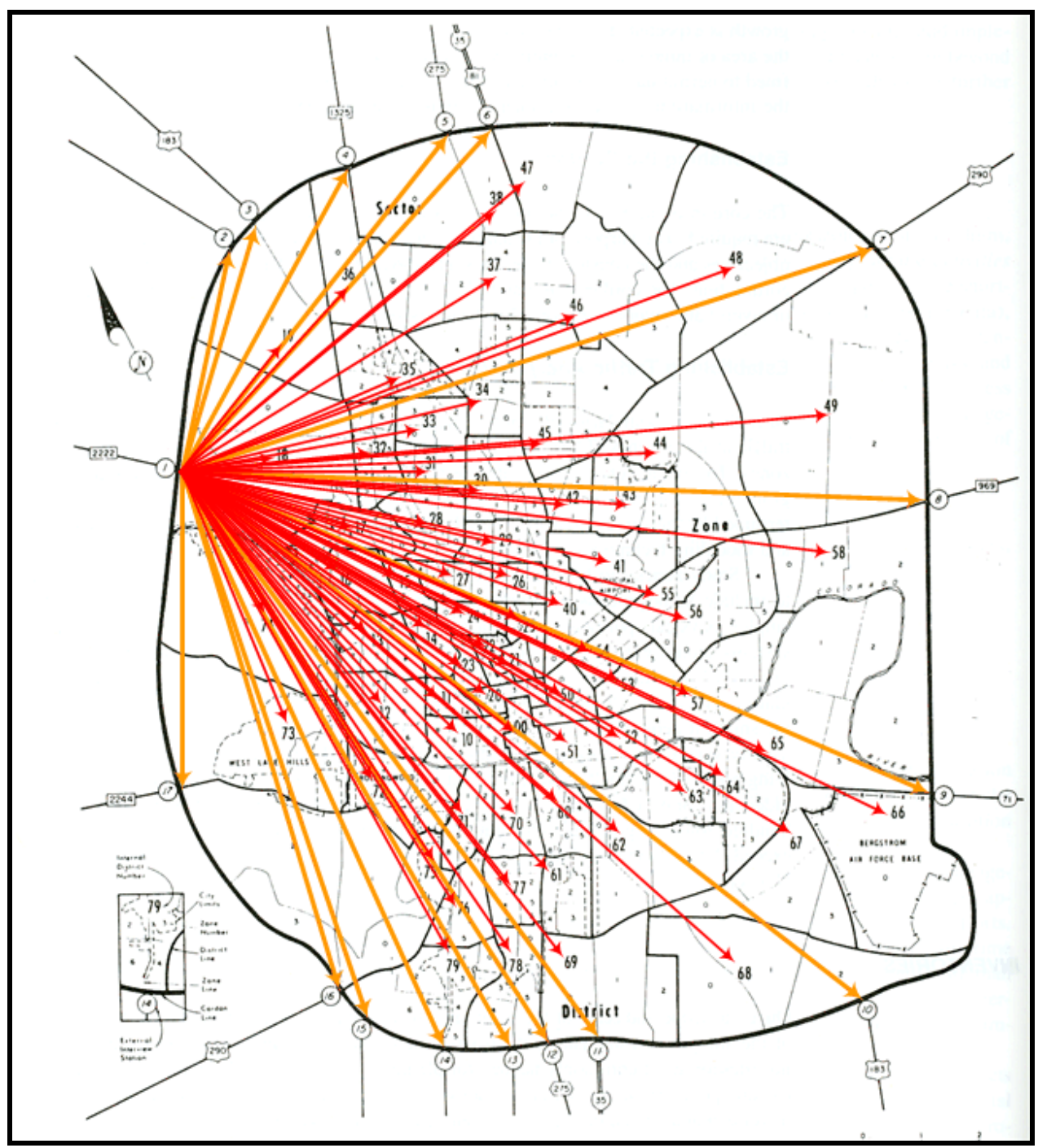

Figure 12: Types of Trips from License Plate Follow-Up Survey Technique

License plates must be recorded roadside using one of the methods described above in the license plate matching section. With this method, however, it is important to record the full license plate of the vehicle in order to contact the proper state motor vehicle department (the Indiana Bureau of Motor Vehicles does not have information on out-of-state license plates) and a partial license plate will not be useful. The advantage over license plate matching is that obtaining each and every passing license plate is not as critical because the plates are not being matched to another observation station, although it is still important to record as many license plates as possible. The license plates will likely have to be transcribed into an acceptable format and sent to each of the respective departments of motor vehicles to obtain addresses. 
Various DMVs may take longer than others to respond, and it may take a long time to get a response from all DMVs. It may be helpful to contact the DMVs in the region ahead of the study. Addresses are usually obtained from the DMV on a cost-perplate basis, which may become quite expensive for large studies. In some states, the DMV may refuse to provide addresses due to privacy issues. For legal information on license plate follow-up surveys in Indiana, see Chapter 4.

In addition, some of the license plates may have been recorded or transcribed incorrectly which will not provide any information. If license plates strings are recorded without using video or photography, the state of the license plate may be difficult to obtain (because the state name is much smaller than the serial number), especially on commercial vehicle license plates, which often have single-color (often white) backgrounds with black or blue characters. Unfortunately, out-of-state vehicles will likely have different travel patterns than in-state vehicles (generally, a higher proportion of outof-state vehicles will be through trips).

Once the addresses are obtained, surveys are usually mailed out to the owners. It is best if this can be done in three to five days, so the trip that is referred to in the survey is still fresh in the mind of the driver. However, it may be that the owner of the vehicle (especially commercial vehicles) was not the driver at the time the license plate was recorded, or the owner may not want to respond to the survey for some reason. Some people may be upset knowing that they were being watched and will not respond due to privacy concerns.

Once the surveys are mailed out, low response rates are usually expected (generally, $15-30 \%$ ). However, providing more than one line of communication (such as internet) may help increase the response. Also, using an internet response may seem easiest, but by doing so, the responding sample will likely be small and unrepresentative of the population of drivers observed on the road. There are many ways the survey responses can become biased. Unlike the license plate matching technique, a lot of time has to be spent reducing returned survey forms. In addition, more money is spent for printing and mailing the survey forms (Quiroga, 2000).

\subsection{Vehicle Intercept Survey Technique}

Unlike the two described above, this technique requires interaction between the driver and observer on the roadway. To conduct an OD study using this technique, 
stations are selected where trip information is desired. All vehicles or a random sample of vehicles are then stopped along the roadway where drivers will voluntarily undergo a roadside interview or be provided with a survey (to be completed after their trip and mailed back). Both methods may be used in the same study (generally when roadside interviews cause backups along the roadway upstream from the interview station), but both methods should not be used on the same driver. Drivers should always be made aware of the upcoming study via warning signs and traffic control barriers, and may be intercepted with the help of police if necessary. Furthermore, an announcement to the general public via radio, television, or newspaper may be appropriate. In some states, this technique may be illegal. For legal information regarding vehicle intercept surveys in Indiana, see Chapter 4. Figure 13 illustrates the typical layout of a vehicle intercept station.

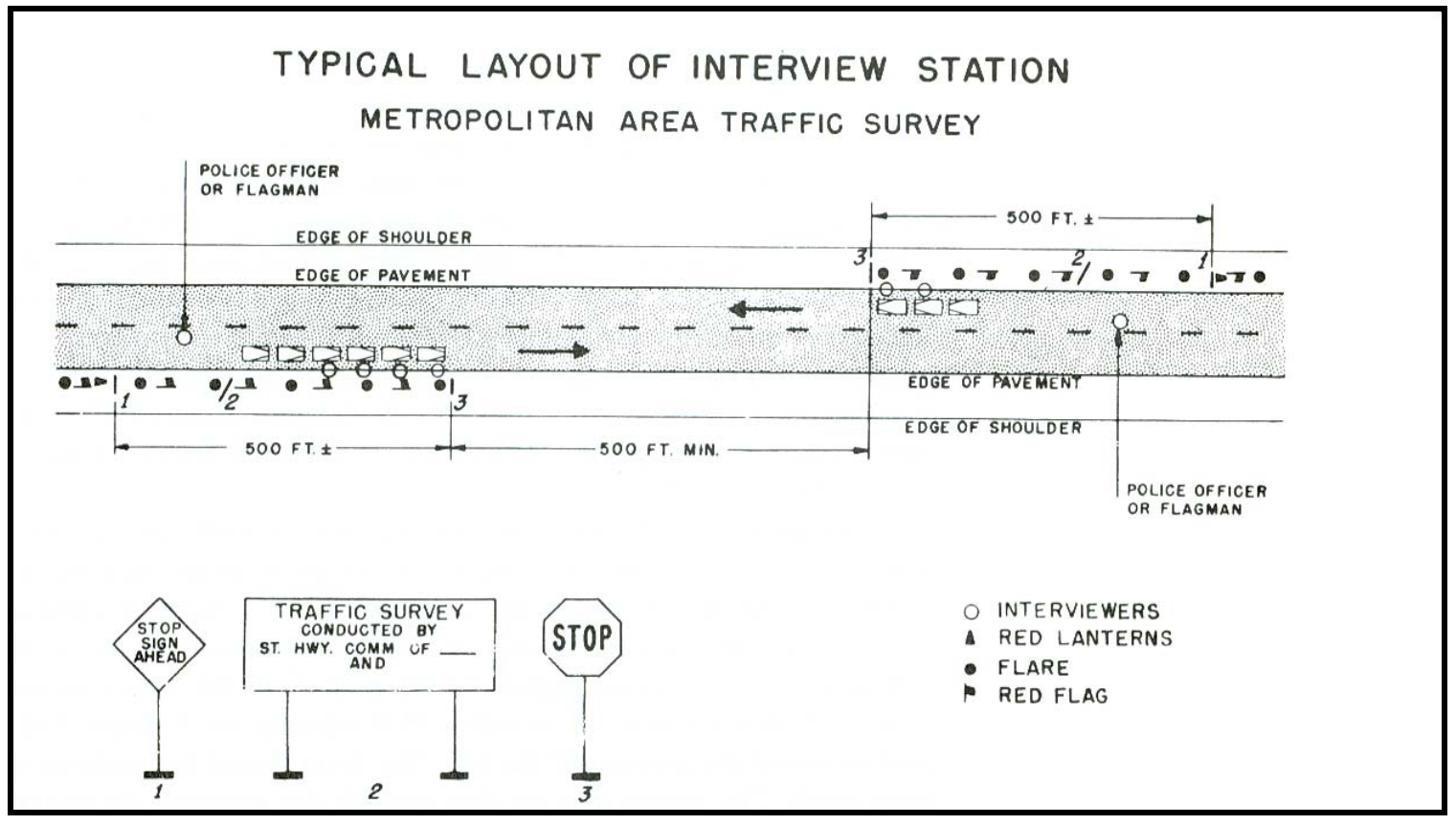

Figure 13: Typical Layout of a Vehicle Intercept Station Source: Robertson, Manual of Transportation Engineering Studies (1994)

This technique is advantageous because it typically yields more trip data than a license plate match. The questions are also adaptable, depending on the circumstances of the study (Virkud, 1995). The response rates are much higher than a license plate 
follow-up survey described in the previous section, and are much less likely to be biased.

This technique is more intrusive to the driver than the license plate matching technique. In addition, vehicles are stopped on the roadway where they interact with interviewers at a station. This can cause delays for the drivers and other traffic and pose a safety hazard for the staff. This technique also requires a lot of manpower in planning and conducting the OD study.

\subsubsection{Roadside Interviews}

Roadside interview is one method of the vehicle intercept technique. Once vehicles are safely intercepted (via a flagger or policeman) and removed from the flowing traffic lanes, the observer conducts an interviewer from the driver's side of the vehicle. This interview should last approximately 30 seconds and not exceed one minute. The interview should seek information about the type of driver, vehicle, and trip. Some vehicle information, such as classification and auto occupancy, may be obtained before or after the interview. The interviewer may use a clipboard or some electronic means (such as a laptop or PDA) to record information. In addition, a map may assist the driver in answering some of the information about the trip. It is important, however, that the map is legible. If the driver is unwilling or reluctant to provide any information, he or she must be let go. For studies in which a sample of drivers is interviewed, a proportional share of vehicle types should be sampled in order to prevent bias.

This type of vehicle intercept survey (VIS) generally yields a great deal of information about one or several specific corridors. Information is collected real-time directly from the person who is driving the vehicle. Most drivers are happy to comply, and driver participation rates are usually above $90 \%$. In addition, a laptop or PDA or other electronic device can be used to store interviews and geocode origins and destinations (Quiroga, 2000).

This method will typically require more manpower than any other technique. Because the observers, supervisors, policemen, and flagmen are interacting with vehicles on the roadway, it is inherently more dangerous than other roadside techniques.

In several states, such as Indiana, roadside interviews (RSIs) have not been used in recent years, primarily due to motorist complaints. However, other factors, such 
as personnel safety, staffing requirements, travel delays, and privacy issues are sometimes cited as reasons for not conducting RSIs.

This method is not suitable for all roadway sections. Traffic volumes on highlevel roadways such as freeways, expressways, and some arterials present a safety hazard to both drivers and interviewers. If, however, rest areas or other easily accessible roadside areas exist in which interviews can be conducted, this technique may be used. The roadside interview is more appropriate in rural areas, particularly along rural two-lane roads. Wherever the interview is conducted, adequate plans for warning signs and traffic control barriers should be drawn up for proper field setup (see Figure 13).

\subsubsection{Postcard Questionnaires}

This method can be used on its own or as a complement to the roadside interview. The handout survey generally asks the same questions as conducted in the roadside interview, however, the drivers voluntarily fill out the information and send the survey back to the agency conducting the interview. The survey should be short enough to fit on a postcard, and return postage should be prepaid. In order to track the types of drivers responding to the survey, color-coded and/or numbered survey forms may be used for each group (vehicle class, out-of-state drivers) in order to expand the data to the entire population.

This method can be used on roadways with higher traffic volumes because they require less interaction time with the driver. Drivers may not have to be directed off the roadway; rather, postcards can be quickly handed out in the traffic lanes to every vehicle as they stop at the roadside station. Like the roadside interview, adequate advanced warning signs and traffic control must be in place for the safety of the drivers and observers.

To complement roadside interviews, this method may be used when backups occur upstream from the interview site. In this case, postcards may be handed to the drivers and they are then permitted to leave. This will eliminate or reduce the delay and number of angry drivers who are stopped to take the interview.

This method can be used as an alternative or in combination with roadside interviews (RSIs). In this method, the same information is generally collected as in an $\mathrm{RSI}$, but the survey is conducted via a postcard that is handed to the driver, completed 
after the trip, and mailed back. A given number of personnel could hand out more questionnaires than conduct roadside interviews.

The problem with this method is the lower response rate than with a roadside interview. In addition, more of the questions may be skipped or answered incorrectly. Generally, response rates for this method are between $15 \%$ and $30 \%$. Furthermore, a lot of time has to be spent reducing returned survey forms, and more money is spent for printing them (Quiroga, 2000). There may be a bias in this type of survey, if nonrespondents (such as certain vehicle types or income levels) have different travel characteristics and demographics than respondents. For example, surveys may not be completed for several reasons: refusal to accept survey, failure to read it, failure to understand it, failure to complete it, and failure to send it back (Bonsall, 1993).

\subsubsection{Tag-on-Vehicle Survey}

In this method, drivers are stopped at roadside stations where a color-coded identifier is placed on the bumper, front window, or radio antenna of passing vehicles. Each roadside station has one unique color assigned to it. Data collectors at each station then record the passing vehicles' tag color (if it has one) to determine the percentage of vehicles coming from another station. Drivers are instructed to remove the identifier at their next destination. With this method, a time stamp will not likely be obtained.

The tag-on-vehicle method is a combination of the VIS and matching techniques. Because the vehicles have to be stopped on the roadway in order for a tag to be placed on their vehicle, it is considered a VIS. However, the tags are monitored as they pass observers through subsequent stations on their trip, so it is also a type of matching technique. The advantages of the tag-on-vehicle method are that it is quicker to conduct than an RSI and easier to match between stations than license plates. However, time stamps may not be collected, unless the vehicle is stopped again at the second station to obtain that information.

On the downside, some motorists may not like the idea of physically attaching a tag to their vehicle, and may disapprove of its placement or remove it before their destination. Still other motorists may leave it attached even after they arrive at their destination, which may cause a significant number of false matches if the vehicle is 
spotted later in the study. In addition, litter could become a problem if tags are not secured or drivers do not dispose of them properly.

\subsubsection{Summary of Methods for the Vehicle Intercept Survey Technique}

Table 2 below lists some of the characteristics of the vehicle intercept survey technique. Each column represents one of the three methods for conducting VIS described in this chapter. The table entries qualitatively assess these characteristics so that each method can be relatively compared with the other methods.

Table 2: Summary of Characteristics for Vehicle Intercept Survey Methods

\begin{tabular}{|l|c|c|c|}
\cline { 2 - 4 } \multicolumn{1}{c|}{} & RSI & PC-Q & TOV \\
\hline Equipment costs & high & med & low \\
\hline Observer training & high & med & low \\
\hline Setup difficulty & high & high & low \\
\hline Subject to weather & med & med & low \\
\hline Power required & yes & no & no \\
\hline Require separate traffic counts & yes & yes & yes \\
\hline Travel Times & no & no & no \\
\hline
\end{tabular}

*Note: RSI: Roadside Interview; PC-Q: Postcard Questionnaire; TOV: Tag-onVehicle

\subsection{Vehicle Tracing Technique}

Vehicle tracing is a technique that utilizes some newer technologies to trace vehicles unobtrusively through a study area. Theoretically, it could be utilized for household travel surveys or the roadside station technique because it would collect travel data on all vehicle trips within a study area including internal-external, externalinternal, and external-external (through) trips. Basically, it obtains the coordinates of vehicles within the study area at regular time intervals. This information is obtained from vehicles equipped with GPS navigation systems or cell phone users. Not only could origin and destination information be obtained, but vehicle paths as well. There are, however, many practical issues that have to be resolved in order to take advantage of this technique. 
This technique is similar to the travel diary technique, but instead of recruiting participants, training them, and distributing sometimes expensive equipment, the data is recorded via technology and infrastructure that is already owned and used by the general public such as cellular phones and GPS systems (such as the On-Star system in GM vehicles). This information could then be obtained from a large amount of people over long periods of time.

This technique is a relatively new technique in that it has only been used in a few small applications, usually to monitor traffic speeds. The biggest disadvantage to this technique is that the much of the public sees it as an invasion of privacy. As a consequence, many of the private companies that provide this service are unwilling to share this information.

In October 2005, the Missouri Department of Transportation contracted with Delcan Corporation and an unnamed wireless carrier to provide real-time, statewide, anonymous cell phone data for monitoring traffic speeds on 5,500 miles of roads in the state of Missouri. Similar, but smaller projects are also underway in Baltimore, Maryland, Norfolk, Virginia, and Atlanta, Georgia (Yahoo! News, October 2005).

\subsubsection{Global Positioning System (GPS) Tracing}

GPS was originally developed by the Department of Defense for military purposes. There are 24 satellites orbiting approximately $11,000 \mathrm{mi}$ above the earth's surface. These satellites can be used to measure location and provide navigational information (driving directions) and time at almost any location on the surface of the earth (Trimble, 2005).

In this method, a GPS-equipped vehicle (such as those with GM's On-Star) records latitude and longitude coordinates at a certain interval throughout its entire trip. This information is then obtained real-time or post-trip (which is similar to the household travel diary method). The data can then be analyzed to develop trip rates, average trip lengths, origins and destinations, etc. for many household types and demographics.

In-vehicle GPS systems such as On-Star were installed for two primary reasons: 1) safety and security such as location tracking for emergency response, roadside assistance, and vehicle theft and 2) navigation and convenience such as driving directions and location-based information and convenience services (OnStar, 2005). The advantage to being able to use this type of system is that it is essentially a GPS- 
assisted travel diary without the need to distribute the GPS equipment. In addition, for each trip, not only are the origins and destinations recorded, but the actual path used by the driver is stored (unlike the clipboard and PDA-assisted travel diary methods).

The disadvantage is that most customers would not approve of being tracked without their knowledge, and it is not known how many would allow their data to be used in an OD study. Furthermore, some information that is obtained via a formal travel diary such as trip purpose and auto occupancy, is not recorded for each trip. Further complicating this matter is the fact that only a small percentage of vehicles are equipped with GPS, and those that do are likely to be owned by households with a higher-thanaverage annual income. Therefore, the sample of vehicles being traced is not likely to be representative sample of the total population.

\subsubsection{Wireless Phone Tracing}

The Federal Communications Commission (FCC) is currently requiring all wireless phone providers to be able to provide location information on all its customers for emergency purposes as part of the E-911 program. The program is supposed to be completed by December 31,2005 , but is running behind schedule. The ultimate goal is to automatically provide emergency dispatchers the location of the wireless phone on which the call is being made within $50-300$ meters (Federal Communications Commission, 2005).

There are two ways wireless phone providers will obtain location information. However, the end results in either case are the coordinates of the wireless phone. One method is handset-based. This method uses a GPS receiver in the wireless phone, which determines location and sends it to the provider. The second method, called network-based, triangulates the wireless phone signal from multiple cell towers to pinpoint the location of the wireless phone (Der Wann, 2002), either by measuring the angle of the wireless signal (angle of arrival - AOA) or the difference in time the signal reaches the various towers (time difference of arrival - TDOA). Currently, both methods are used in the US. Of the major US wireless carriers, Verizon and Sprint use the handset-based method, while Cingular and T-Mobile use the network-based method. Like the GPS probe vehicle method, the location information obtained can then be plotted to a GIS package to reveal travel patterns of persons carrying cell phones. 
Figures 14 \& 15 illustrate the difference between the handset-based and network based location determination technologies.

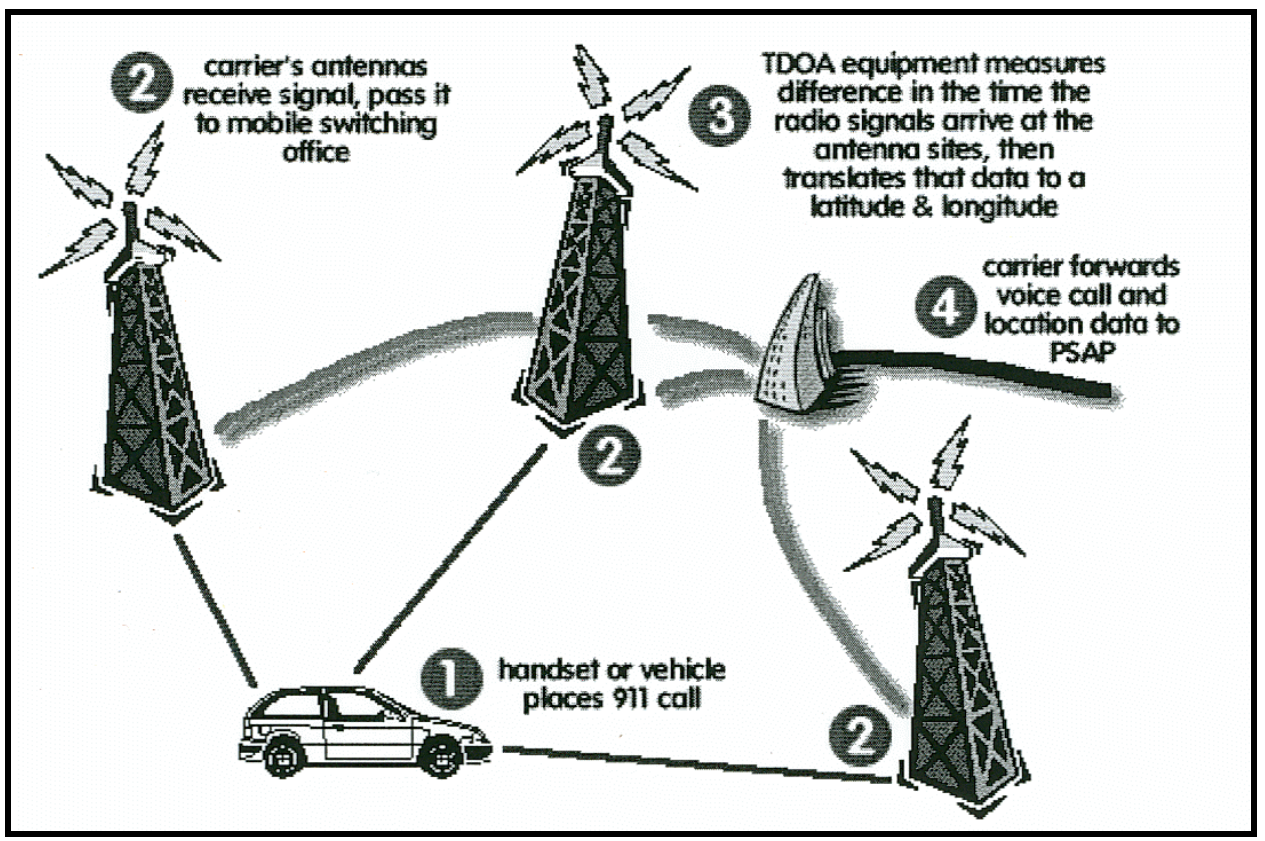

Figure 14: Handset-Based Location Determination using GPS Note: PSAP - Public Safety Answering Point

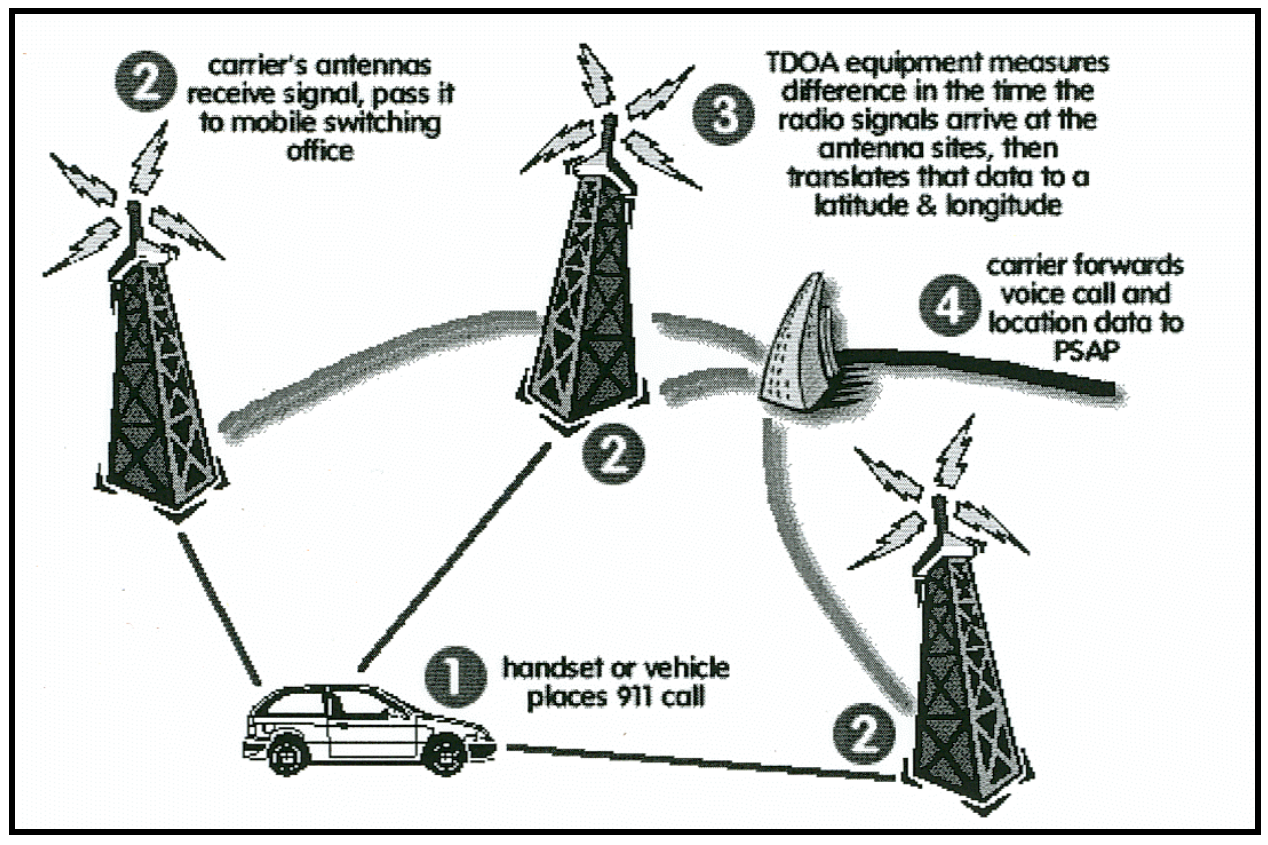

Figure 15: Network-Based Location Determination using TDOA Source: www.911dispatch.com 
The wireless phone tracing method has many of the same advantages and disadvantages as the GPS method described in the previous section. The biggest advantage to cellular tracing compared to GPS is that there is a much higher market penetration. At the end of 2004, there were 182 million cell phone subscribers in the US, up 23.4 million from 2003 (Cellular Telecommunications and Internet Association, 2005). Cell phone ownership is likely to be more equally spread among the different cohorts of age, income, and household size than GPS-equipped vehicles. Like GPS tracing, cell phone tracing can also provide the actual routes of moving people rather than just the origin and destination as in a clipboard travel diary or license plate match.

There are some disadvantages with this method. Information such as trip purpose, auto occupancy, and possibly travel mode may be unobtainable. In addition, most cell phone owners, like GPS-equipped vehicle owners, would not want to be traced without their knowledge. However, due to the deep market penetration of cell phones, it may be likely that there are enough volunteers to complete a study like the travel diary technique. In addition, while the FCC is trying to implement Phase II of the E-911 program, it has not been completed in every state and county. Furthermore, the accuracy of the locator points is not as accurate as with GPS, and depending on the technology used by the wireless carrier, location information may not be provided for some phones located in vehicles (because they need a clear view of satellites). Finally, by tracing cell phones, person-trips, not vehicle trips are being recorded. This may or may not be advantageous. For vehicle trips, it is possible that there could be multiple cell phones in the same vehicle (such as a bus). Likewise, phones not in vehicles (for example, pedestrians on sidewalks) will have to be filtered from those that are. On the other hand, all travel modes could be traced, which would aid in developing activitybased travel demand models.

\subsubsection{Summary of Methods for the Vehicle Tracing Technique}

Table 3 below lists some of the characteristics of the vehicle tracing technique. Each column represents one of the three methods for conducting VIS described in this chapter. The table entries qualitatively assess these characteristics so that each method can be relatively compared with the other methods. 
Table 3: Summary of Characteristics for Vehicle Tracing Methods

\begin{tabular}{|l|c|c|}
\cline { 2 - 3 } \multicolumn{1}{c|}{} & GPS & Cell \\
\hline Equipment costs & high & high \\
\hline Implementation Time & high & high \\
\hline Subject to weather & no & no \\
\hline Require separate traffic counts & yes & yes \\
\hline Travel Times & yes & yes \\
\hline Vehicle Paths & yes & yes \\
\hline
\end{tabular}

\subsection{Mathematical OD Estimation Techniques}

Over the years, a lot of research has been conducted on developing origindestination matrices using mathematical techniques to avoid the often time-consuming and costly attributes of conducting a formal OD study, especially in large urban areas. Most of these techniques rely on link traffic counts and some knowledge of a prior matrix, usually one from a previous study. However, the number and location of traffic counts, the quality of the prior OD matrix (in many cases, there isn't one) often influence the quality of the newly estimated OD matrix. In addition, many have additional constraints that make them valid under certain assumptions (e.g., simple, uncongested networks).

While there are many techniques available, no single estimation technique has emerged that is widely accepted in practice. Therefore, these estimation techniques were not evaluated as part of this project.

\subsection{Summary of All OD Techniques}

Each of the techniques and methods for conducting a Roadside Station OD Study, the characteristics of each, and their advantages and disadvantages have been described in detail in this chapter. In order to better compare those aspects, Table 4 summarizes the characteristics of the Roadside Station OD Study and qualitatively compares each of the techniques. 
Table 4: Summary \& Comparison of Roadside Station OD Study Techniques

\begin{tabular}{|c|c|c|c|c|c|c|c|c|c|c|c|c|c|}
\hline & 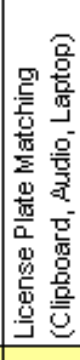 & 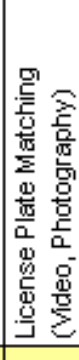 & 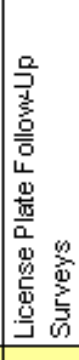 & 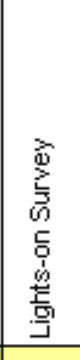 & 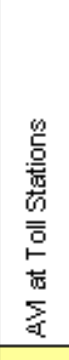 & 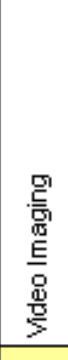 & 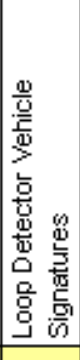 & 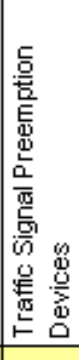 & 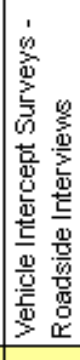 & 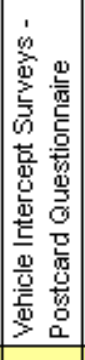 & 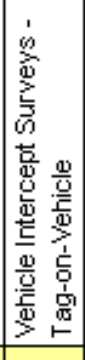 & 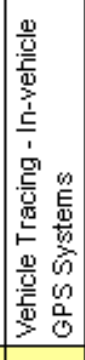 & 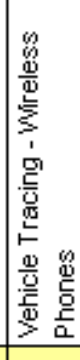 \\
\hline Roadside Data Collection & yes & yes & yes & yes & no & yes & no & no & yes & yes & yes & no & no \\
\hline Third Parties Involved & no & no & yes & no & yes & yes & yes & yes & yes & yes & no & yes & yes \\
\hline Interrupt Traffic Flow & no & no & no & no & no & no & no & no & yes & yes & yes & no & no \\
\hline Intrusive to Driver & no & no & yes & no & no & no & no & no & yes & yes & yes & no & no \\
\hline Location Setup Flexibility & yes & yes & yes & yes & no & no & no & no & no & yes & yes & yes & yes \\
\hline Mass Phone Calls, Mailings & no & no & yes & no & no & no & no & no & no & yes & no & no & no \\
\hline Lots of Personnel Required & yes & yes & yes & yes & no & yes & no & no & yes & yes & yes & no & no \\
\hline Police Required & no & no & no & no & no & no & no & no & yes & yes & yes & no & no \\
\hline Technology Proven & yes & yes & yes & yes & yes & no & no & no & yes & yes & yes & yes & no \\
\hline Mass Distribution of Equipment & no & no & no & no & yes & no & no & yes & no & no & no & no & no \\
\hline Human Error Possible & yes & yes & yes & yes & no & yes & no & no & yes & yes & yes & no & no \\
\hline Conventional Technique & yes & no & yes & yes & no & no & no & no & yes & yes & yes & no & no \\
\hline Large Sample Sizes Obtainable & no & yes & no & yes & no & yes & yes & no & yes & no & yes & no & yes \\
\hline Privacy Concerns & no & no & yes & no & yes & yes & no & no & yes & yes & yes & yes & yes \\
\hline Easy to Implement & yes & yes & yes & yes & yes & no & no & no & no & no & no & no & no \\
\hline Fatigue for Personnel & yes & no & yes & yes & no & no & no & no & yes & yes & yes & no & no \\
\hline Maximum Traffic Speed & low & high & high & high & high & high & high & high & med & med & med & high & high \\
\hline Maximum Traffic Flow & low & high & high & high & high & high & high & high & med & high & med & high & high \\
\hline Permanent Record of Data & yes & yes & yes & no & yes & yes & yes & yes & yes & yes & no & yes & yes \\
\hline Subject to weather & yes & yes & yes & no & no & yes & no & no & yes & yes & yes & no & no \\
\hline Continuous data collection & no & no & no & no & yes & no & yes & yes & no & no & no & yes & yes \\
\hline Participation Recruitment Required & no & no & yes & yes & yes & no & no & yes & yes & yes & yes & no & no \\
\hline Travel Times Precision & med & high & low & none & high & high & high & high & low & low & none & high & high \\
\hline Personnel Training Required & yes & yes & yes & yes & no & yes & no & no & yes & yes & yes & nia & no \\
\hline Data Reduction Time & med & high & high & low & med & high & high & med & high & high & low & med & med \\
\hline Obtain True O\&D & no & no & yes & no & no & no & no & no & yes & yes & no & yes & yes \\
\hline Obtain Trip Purpose & no & no & yes & no & no & no & no & no & yes & yes & no & no & no \\
\hline
\end{tabular}




\section{CHAPTER 3 - STATE DOT SURVEYS ON OD TECHNIQUES AND METHODS}

To determine the extent of the use of the different types of OD techniques and methods by other state DOTs, two surveys were prepared and sent to representatives from each of the state DOTs. The surveys and the results are described below.

\subsection{Survey on Roadside Interviews}

In November 2004, a short survey was sent to the Highway Performance Monitoring System (HPMS) coordinators at each State DOT to determine the usage and legalities of roadside interviews (RSIs) in each state. The questions asked were: "/s your DOT permitted to stop traffic to ask motorists questions such as their origins and destinations? If so, to what extent do you use this travel survey method?" The HPMS coordinator was asked to forward the question on to the appropriate person if he/she was not able to provide the answer.

14 of the 50 states responded. All of the states responding said roadside interviews were legal in their state, but only seven of the states said they are used (and rarely used in three of those states). State DOTs that do conduct RSIs include Delaware, South Dakota, Michigan, New Jersey, Ohio, Mississippi, and Utah. State DOTs that do not conduct RSIs include New Hampshire, Wyoming, Connecticut, Pennsylvania, Colorado, Rhode Island, and New Mexico (although consultants may conduct RSIs in the New Mexico from time to time).

Of the states that do conduct RSIs regularly, most do with the assistance of the police department. In Florida, however, it was the police department that decided to stop participating in RSIs as a result of motorist complaints (Florida Highway Patrol, 2005).

Some states, like Delaware and Utah, prefer the postcard questionnaire method over RSIs. While Delaware has conducted RSIs on freeway mainlines, most now are 
done via postcard during red phases on arterial streets. In New Mexico, RSIs can only be conducted in rest areas.

The Michigan DOT, however, has revived the use of RSIs in the state with the approval of the Attorney General's Office and the Traffic and Safety Division, and has used them frequently in the past two years. In 2004, 19 RSIs were conducted, interviewing 25,000 drivers and generating only two dozen complaints. Michigan has used this method on both two-lane and multi-lane highways with ADTs less than 30,000 vehicles. They have stopped motorists near toll facilities, rest areas, and on the mainline. Postcard surveys were also conducted in some locations with a $25 \%$ response rate.

Even though RSIs are legal in all of the states surveyed, most DOTs rarely or never perform them.

\subsection{Survey on all OD Techniques and Methods}

To obtain more data regarding all the techniques, a survey of the 50 State DOTs was conducted in April 2005 to determine the state-of-the-practice regarding policies and use of data collection methods for origin-destination studies. The survey was distributed to state DOTS via a contact list provided by the American Association of State Highway and Transportation Officials (AASHTO). The survey is shown in Appendix 1 as it was sent.

The initial deadline was set about six weeks after the survey was distributed. The survey was sent again in its same form after the initial deadline to encourage more response. Ultimately, a total of 19 states responded to the survey.

\subsubsection{Results}

- Does your state DOT have any written guidelines for conducting origin-destination studies (for cities and towns located outside the jurisdiction of an MPO)? If so, how could I obtain a copy of those guidelines?

Four out of 17 states (24\%) utilized some form of written guidelines, ranging from past experiences to TMIP documents. 
- Does your state DOT have a preferred data collection method for conducting origindestination studies (whether completed in-house or by a consultant)? If so, what is that method?

Four out of 17 states (24\%) have preferred method (one state indicated two) for collecting OD data. Of the four states:

- Three indicated VIS

- One indicated LP matching with video

- One indicated LP follow-up surveys

- If your state DOT or its consultants have conducted any origin-destination studies in the last five years, please complete sections A - F below. If not, skip to question 6 .

Twelve of the 19 states (63\%) indicated they have conducted at least one OD study in the last 5 years. The following list indicates how many states utilized each technique and method.

\section{A. Roadside License Plate Matching Technique}

Six of the twelve states $(50 \%)$ used this technique:

- One state used the clipboard method

- Two states used the audio method

- One state used the laptop method

- Three states used the video method

B. Roadside License Plate Follow-Up Survey Technique

Four of the twelve states (33\%) used this technique:

- All four states used the video method (three with manual transcription, one with automatic transcription)

- All four states contacted vehicle owners by mail

C. Vehicle Intercept Survey

Eleven of the twelve states (92\%) used this technique:

- Ten states used the roadside interview method

- Seven states used the postcard handout/mail-in method

D. Travel Diary

Eight of the twelve states $(67 \%)$ used this technique:

- All eight states used paper travel diaries (two were part of the 2001 NHTS) 


\section{E. Recall Interview}

Two of the twelve states (17\%) used this technique:

- Two states contacted households via telephone

- One state contacted households via mail

\section{F. Vehicle Tracing}

One of the twelve states (8\%) used this technique. The method was neither GPS nor cell phone tracing (a special study was created in which random vehicles were followed to their destination).

- Is there any particular data collection method (not limited to those listed above) your state DOT has utilized that has met or exceeded your expectations in terms of time, cost, accuracy, etc? Please explain.

Four of the 19 states $(21 \%)$ indicated one method exceeded their expectations:

- Three states indicated vehicle intercept surveys

- One state indicated LP matching with video

- Are there any methods that have failed to meet your expectations? Please explain.

Four of the 19 states $(21 \%)$ indicated one method failed their expectations:

- Two states indicated LP matching with video

- One state indicated LP follow-up surveys

- One state indicated roadside interviews.

\subsubsection{Conclusions}

Several conclusions can be drawn from this survey. First, most states do not have any sort of guidelines to refer to when conducting origin-destination studies. Several of the responses indicated they were interested in obtaining any guidelines that result from this study. Secondly, a wide variety of techniques have been used by the responding states, with the most common to being vehicle intercept surveys, which seems to indicate that, if conducted correctly, the VIS provides good OD information. Finally, techniques that exceeded one state DOT's expectations failed another's (vehicle intercept surveys and license plate matching with video were mentioned in each of these questions). This may mean that certain techniques were conducted in situations that provided poor results. 


\section{CHAPTER 4 - LEGAL ISSUES OF VEHICLE INTERCEPT AND LICENSE PLATE FOLLOW-UP SURVEYS IN INDIANA}

Vehicle intercept surveys have not been conducted in the state of Indiana since 1991 due to an incident on an Indiana freeway that prompted a motorist complaint. While the Indiana Attorney General intervened, it was unknown by INDOT officials if VIS were officially made illegal or if the intervention applied to this single incident.

Likewise, license plate follow-up surveys have not been conducted in Indiana in recent years. Critics of this technique often state that obtaining the vehicle owner's address from the motor vehicle bureau is an invasion of privacy. During this study, a call to the Indiana BMV was made to inquire about obtaining information from a license plate survey for OD purposes. The BMV responded that under no circumstances would personal information (including owner addresses) be provided, even to other Indiana government agencies.

To answer these questions, the Indiana Attorney General is being contacted with assistance from the INDOT legal department for a clear and final ruling on the current and future status of vehicle intercept and license plate follow-up surveys in Indiana. This information will be provided under separate cover. 


\section{CHAPTER 5 - EVALUATION OF EQUIPMENT FOR LICENSE PLATE DATA COLLECTION}

The purpose of this chapter is to evaluate the technology and equipment used in various methods of recording license plate strings (either 4-character or full strings) from a roadside station. These strings can then be matched with a list of license plate strings obtained at other roadside stations (the license plate matching technique), or the strings can be used to obtain addresses of vehicle owners to which a survey can be mailed seeking specific trip information (the license plate follow-up survey technique).

\subsection{Clipboard Technology \& Equipment}

For this method, very little equipment is required. Each observer needs a clipboard with forms on which to record the license plate strings. Also required is a device to keep time (clock, stopwatch, or wristwatch), which should be synchronized with all other stations when conducting a study. A sufficient supply of writing utensils should be provided as well.

Tablet PCs are an alternative to the standard clipboard if automatic transcription of the license plates is desired. Tablet PCs may look much like a typical laptop; however, the unit can be closed with the screen facing outward, which then becomes an electronic clipboard. Handwritten information can be written directly on the screen using a special writing pen. Tablet PCs have handwritten character recognition software that converts handwritten text into digital characters in either real-time or upon command. However, the accuracy of this software and its adaptability for roadside license plate data collection is unknown, and it was not tested as part of this study. At this time, most tablet PCs cost anywhere from $\$ 1000$ to $\$ 2500$ each. 


\subsection{Audio Technology}

There are basically two types of audio recorders: the traditional cassette tape recorders and tape-free digital recorders. Both types were analyzed in this project to determine if one type was better suited for license plate data collection.

\subsubsection{Analog Cassette Audio Recorders}

Most people are familiar with this type of audio recorder. The controls and functions on it are simple and easy to understand. The recorder features two recording modes: low speed and high speed. On the low-speed "normal" setting, the cassette tape lasts as long as the time printed on the cassette itself. For example, a 60-minute cassette can store 60 minutes of data (30 minutes per side). The high-speed setting records a higher quality of sound, however, the cassette can only record for half the time printed on the cassette. For example, a 60 -minute cassette can store 30 minutes of data (15 minutes per side).

Some audio cassette recorders have a voice-operated recording (VOR) feature, also known as voice-activated recording. When the recorder senses no sound in the room, the tape will automatically pause after a few moments, and resume once sound returns. If the recorder is being used along the roadside, the background noise from passing vehicles (especially on high volume roads) is usually enough to prevent the VOR from pausing in the first place, which defeats the purpose for which it was intended. Instead, if it is desired that long periods between vehicles are not recorded, the pause button can still be used manually. However, it is important for the observer to remember when the recorder is paused so that recording can begin again when the next vehicle approaches. In addition, the time should also be updated during delays between vehicles, usually no less than every one or two minutes.

\subsubsection{Digital Audio Recorders}

Digital recorders differ from cassette recorders for a number of reasons. First, there is no external storage medium in a digital recorder. Rather, the data is stored internally, usually in .wav files. The data is stored in files, so recordings are organized much like songs on a music $C D$. The files can also be downloaded to a computer, where the information can be digitally manipulated and stored. 
Like the cassette recorder, digital recorders typically have several recording modes. Similarly, the higher the quality of the recording, the faster the storage is used.

The recorder may also have a microphone sensitivity adjustment. Usually, there is a setting for dictation and another for recording sounds in all directions. For recording license plates along the roadside, the microphone sensitivity should likely be set on the dictation setting to minimize the amount of background noise that is recorded, which can reduce the quality of the license plate records during playback.

Compared to the cassette recorder, the digital recorder is a little more complicated than the cassette recorder to run at first, mainly to learn how the file storage system works. Because there is no external storage medium like a cassette, the data has to be deleted or downloaded when it becomes full. For a long OD study, the data would have to be downloaded several times during the study period (which requires a laptop and time away from continuously recording plates) or a multiple recorders at each station.

Like the cassette recorder, digital recorders may also have the VOR feature, although it may have a different name. For either recorder, it is recommended that this feature be turned off.

\subsection{Audio Equipment}

Two handheld audio recorders, one analog and one digital, were purchased for this study. The features of each are described below, followed by an evaluation of each after being used to record license plates at a roadside setting. It should be noted, however, that audio can also be recorded to other devices, such as a laptop computer, which may be done if speech recognition software will be used to transcribe the license plates in real time. Speech recognition technology was not evaluated as part of this project. However, Washburn (1997) was able to obtain over 95\% accuracy when recording 525 vehicles per hour in field tests.

\subsubsection{Sony Micro-cassette Audio Recorder}

The first recorder, a Sony M560-V, is an analog micro-cassette recorder. It was purchased for $\$ 33$. The recorder features two recording speeds, the faster of which produces higher-quality recording. It also has voice-operated recording (VOR), which is used to save cassette space and battery power by automatically stopping recording 
when noise levels are minimal. The VOR sensitivity feature can be set on high, low, or off. The recorder also contains a tape counter and one-finger rewind, fast-forward, and pause for easier review during playback. The recorder runs on $2 \mathrm{AA}$ alkaline batteries or an AC adaptor (not included). The microphone is built in on the end of the unit (separate from the playback speaker). This particular model does not have a separate microphone jack, but it can be found on other Sony models. An earphone jack and earphones are also included. The dimensions of the unit are $21 / 2 " \times 4 \frac{3 / 4 "}{\prime \prime} \times 1 "$, and it weighs $4.0 \mathrm{oz}$. A 4-pack of standard micro-cassettes with $60 \mathrm{~min}$ of recording time per cassette costs less than $\$ 5$.

\subsubsection{Olympus Digital Audio Recorder}

The second recorder is an Olympus VN-240PC digital voice recorder. It was purchased for $\$ 69$. Rather than record to cassette tapes, recordings are stored in .wav files in four folders (A, B, C, S) for easier management. Each folder can hold 100 files. Associated automatically with each file are the recording date and time, recording time length, and audio quality. Files can be sorted, stored, and played back via the display screen on the recorder.

The Olympus model has three recording modes: LP (lowest quality), SP, and HQ (highest quality). The recorder can store a maximum of 245 minutes, 133 minutes, and 88 minutes, respectively, for each recording mode. There are also two levels of microphone sensitivity: high - to be used to record sound from all directions, and low for dictation use. Like the VOR on the Sony model, this model contains variable control voice actuator (VCVA), which can be turned on or off. Index marks (up to 10 per file) can also be set while a file is being recorded. These can be used to find important times in the file during playback. The recorder also contains microphone and earphone jacks, although the accessories are not included.

This model also contains software to transfer audio files to a PC. A USB cable is included. The Windows-based software can be used for storage and management of recordings, playback, and direct recording to a PC. The recorder does not have a conventional pause button. Instead, if recording is stopped and resumed, the resumed portion is recorded into a new file. This model has a maximum of 100 files, regardless of whether all the recording time has been used up. The dimensions of the unit are $3 / 4$ " $x$ $1 \frac{1}{2} " \times 3 / 4$, and it weighs just $2.4 \mathrm{oz}$. 


\section{$5.4 \quad$ Evaluation of Audio Equipment}

Because it is necessary to stand as close to the roadway as is safely possible in order to see license plate information, there is generally a significant amount of background noise due to the traffic itself. This noise also increases as the speed of the vehicles increase. Therefore, it is important to make sure that, when using an audio recorder to collect license plate data, the noise from the traffic does not prevent the data on the audio recorder from being heard clearly during playback.

In order to compare the performance of the recorders, field testing was conducted on the shoulder of a high-speed, four-lane highway. The results of the testing of various features of the recorders were compared and are discussed below.

\subsubsection{Audio Quality}

As stated in the previous section, both recorders have different settings that regulate the quality of the data recorded. For the cassette recorder, the faster the tapes run, the higher the quality of the recording (and lower the storage capacity). Likewise, while there is no "tape" in the digital recorder, higher quality is traded for lower capacity.

Tests were conducted in the same location on the roadside using two audio recorders. License plate data was recorded on each of the settings and later reviewed to determine if any of the data was indecipherable. The recorders were tested using the manufacturers suggested settings for microphone sensitivity (if applicable), and each recorder was placed the same distance (approximately 1 foot) from the observer. The use of microphones or headsets were not evaluated on either type, rather, license plate data was spoken directly into the microphones built into the recorders.

For both recorders, the highest quality audio setting is significantly better than the lower settings. In situations with lots of background noise, the recording becomes "scratchy" and reduces the clarity of the voice recording. Therefore, it is recommended that the audio settings of the recorder be set on the highest quality to avoid loss of license plate data.

\subsubsection{Voice-Operated Recording}

Both the recorders tested as part of this project have voice-operated recording. The idea behind this feature is to preserve storage space by automatically stopping the 
recording when there is no sound present. On the roadside, it is unlikely, especially on high-flow roads, that there will ever be a long enough silence to activate this feature. The recorders were tested, however, to determine if this feature should be turned on or off during a study.

For the cassette recorder, this feature should be turned off. This is because recording resumes by an observer speaking into the recorder, the first part of the data spoken into the recorder is lost. For example, if an observer speaks "A123" into the recorder, only " 123 " is heard during playback.

The digital recorder, on the other hand, did not have the same problem as the cassette recorder. However, it is unknown if all digital recorders are able to resume recording without cutting off the first piece of information. Therefore, it is recommended that VOR not be used on either type of audio recorder during an OD study. Because audio recorders will likely be used in noisy situations, the benefit of VOR is probably minimal.

\subsubsection{Storage and Power}

Because the high-quality audio settings are recommended to be used, the storage capacity of each recorder is reduced. For the analog cassette recorder, the micro-cassettes can record 15 minutes of data per side. This particular digital audio recorder can record 88 minutes of data on the high-quality setting. The problem with the digital recorder is that there is no external storage medium. The data can be recorded directly to a laptop in real-time (this is beneficial if speech recognition software will be used to automatically transcribe the data). Otherwise, the data must be downloaded when all of the storage is used, which also requires a laptop in the vehicle. With the analog cassette recorder, however, a new tape can be inserted relatively quickly.

Both recorders run on AA alkaline batteries. For long studies, the batteries may need to be replaced. This task can be coordinated and planned among the observation stations ahead of time, and can be completed in a relatively short amount of time.

While both recorders have advantages and disadvantages, the cassette recorder is recommended for use in an actual OD study with manual transcription of the license plate data. Compared to a digital recorder, the quality of the audio is similar, it is easier to operate, and it has external storage that can be changed relatively quickly and easily. 


\subsection{Laptop Technology \& Equipment}

The laptop method requires a laptop computer or some other electronic device in which the license plate string can be keyed in using a standard keyboard. No specialized software is required to store the license plate strings. Microsoft Office software (Excel or Access) is likely to suffice. However, if more accurate time stamps are desired for each license plate string, other software could be used. A simple data entry form (for the license plate information) could be created in Access that contains a macro that time stamps each license plate string with the current time as they are being entered into the computer.

For this project, a Dell Latitude C600 was used. However, almost any type of laptop could be used. For the purposes of this study, license plates were recorded in Microsoft Excel, although another self-written program could be used to automatically time stamp each license plate entry, store the data, and later, match the data.

There are a couple of important issues to consider when using a laptop on the roadside. First, depending on the length of the study, the laptop may require additional power beyond that of its regular battery. This may be achieved by using a higher capacity battery or an adapter to connect to an automobile power source. Secondly, the visibility of the screen may be reduced due to glare produced by the sun. This may affect the quality of the license plate data being entered by an observer. Finally, most laptops do not have a separate number keypad (as found on most desktop computers). The amount of time it takes to enter a series of digits will likely be longer if a number keypad is not available.

\subsection{Digital Video Technology}

A lot of license plate data collection has been conducted using analog camcorders for traffic data collection. In recent years, however, digital video technology has advanced well beyond VHS analog technology, and prices continue to fall. In 2005, digital camcorders cost as little as $\$ 300$. In addition, many manufacturers have stopped producing analog cameras altogether. However, it is important that digital camcorders can be easily utilized to produce adequate-quality video for use in OD studies. So what exactly are the benefits and characteristics of digital video that make it more appealing? The following sections discuss some of those characteristics of digital video and identify 
important features to look for when purchasing digital camcorders for the purpose of recording license plates on the roadside.

\subsubsection{Resolution}

Resolution is one of the primary benefits of digital video. While $8 \mathrm{~mm}$ analog (VHS) camcorders have up to 250 lines of resolution, digital cameras have up to 520 lines, although the actual resolution is likely to be lower and varies from model to model. Hi8, a higher-quality $8 \mathrm{~mm}$, can achieve only 400 lines. Furthermore, digital video quality is not reduced by making copies or in storage over time, and editing is much easier.

\subsubsection{Recording Media}

Digital cameras currently have three types of recording media: tape, DVD, and flash. MiniDV digital videotape is currently the most common. It is produced by almost all manufacturers. (Sony, however, uses a digital tape format called Digital8.)

DVD camcorders have also become popular in recent years. These camcorders record on smaller-sized DVDs in one of three formats: DVD-R, DVD-RW, or DVD-RAM. A good feature of DVD recording is that editing can be done directly on the camera. However, not all DVD players (especially older ones) can play the DVD-RW and DVDRAM formats, and recording time on one DVD is less than that of a MiniDV.

The other type of media is flash memory, such as SD or Compact Flash. Most camcorders use this type for still digital pictures in addition to the tape or DVD media. However, some camcorders record video to flash as well, although it hasn't really caught on, and storage capacity is much smaller than with MiniDV or DVD.

\subsubsection{Playback \& Editing}

Digital video cannot be played back through a standard VCR like VHS tapes. Instead, digital video can be played to a TV directly from a camcorder, or the video can be downloaded to a computer for editing and storage. The best way to transfer video is through the IEEE 1394 serial bus, more widely known as a FireWire (although it is also called iLink by Sony). Most camcorders produced today have this port; however, not all computers do, especially older models. A newer, high-speed USB port, USB 2.0, is also capable of video transfer. Once the video is transferred to a computer, it can be edited 
and burned to DVDs for storage. If no editing is desired, it can always be stored on the MiniDV tape itself. These currently cost about \$5-7 per (one-hour) tape.

Some of the more technical details of a digital camcorder for recording vehicle license plates for an OD study include color, magnification, focus, shutter speed, and exposure. These are discussed in greater detail below.

\subsubsection{Charge-Couple Device (CCD)}

For the best color, the CCD size and number are important. CCD stands for charge-couple device, or more simply, the "chip". The CCD is the digital equivalent of film. Its size is one of the biggest factors in the price of a digital camcorder. Sizes in consumer-grade camcorders typically range between 1/6" to 1/3", although professionalgrade cameras have up to $2 / 3$ " or 1 " CCDs. CCD size is most important in low-light situations. Larger CCDs also have more pixels, which allow the picture to be enlarged without pixilation. However, above 340,000 pixels, the sharpness of the image improves at a decreasing and negligible rate.

Another difference between camcorders is that it may contain one or three CCDs. In three-CCD camcorders, there is one chip for each of the primary colors. Camcorders with three CCDs have brighter and more lifelike colors than one-CCD camcorders, and cannot presently be found on the market for less than $\$ 800$.

\subsubsection{Magnification (Zoom)}

Camcorders typically have both optical and digital zoom. Optical zoom is an actual movement of the lens of the camera and typically has magnification up to $25 x$. Digital zoom, however, is just the enlargement of pixels, and will result in pixilation. Most camcorders boast digital zooms of $500-1000 x$, but it is largely a marketing tool, as the image will be completely illegible at such a high digital magnification.

\subsubsection{Focus}

Focus is an important part of recording video, especially for license plates. Camcorders usually have automatic focus and manual focus options. For manual focus, the best type of manual focus is a focus ring, which is usually located around the lens. Some camcorders have a jog dial (similar to a sliding switch) or a focus button. Sony 
has a "spot focus" feature, where the user can use the touch-screen LCD viewfinder to place the focus on a specific object.

\subsubsection{Shutter Speed}

Shutter speed is a measurement of the opening of the camcorder's shutter in cycles per second. Low shutter speeds admit more light to the CCD than high shutter speeds and will result in a blurring of fast moving objects such as vehicles or athletes. Too little light admittance at a high shutter speed can be compensated by adjusting the exposure (or aperture). In some models, this adjustment is done automatically.

\subsubsection{Exposure/Aperture}

While the shutter controls the interval in which light is admitted, the exposure or aperture controls the amount of light during any given interval. The bigger the aperture, the more light is let in, which results in a brighter picture. Because most consumers (and transportation professionals) are not photography experts, most camcorders produced today have an automatic mode that controls the exposure. Many also have programmed auto exposure (and shutter speed) modes such as "sports", "candlelight", "spotlight", "snow", etc. (easycamcorders.com, 2005)

\subsubsection{Other Features}

Over the course of conducting this research with several different camcorder models, there were a couple other features that were found to be helpful, but not available on every model. The first, slow motion (and frame-by-frame) playback, was available on only one model. This feature was very helpful for analyzing video of highspeed vehicles that only appeared on the viewfinder for fractions of a second. For some of the models, there is a slight delay when the video is paused. Because of this, it was very difficult to actually stop the video when the license plate was in the field of view, in which case, the video had to be rewound, replayed, and paused repeatedly until the desired frame appears still on the screen. This process could take several tries, which will result in longer analysis time and frustration for the observer. With slow-motion and frame-by-frame capabilities, the observer is able to slow down the video as the front of the vehicle entered the screen, then step forward (or backward) until the desired frame appears in the screen. This camcorder model also had a remote control from which the 
video playback was controlled. If multiple camcorders are used in a study, not all need to have slow-motion capabilities. Instead, the one camcorder with these features can be used to play back the video recorded on all other camcorders.

Another feature that was only available on one of the camcorders was a time stamp that was precise to the nearest second. While all models typically measure the location of the tape in an hour, minute, second, and frame format, this information can only be shown on the viewfinder, but not the television screen during playback. The information that can be viewed on the television screen during playback is the actual date and time. However, only one of the models measured the time to the nearest second (the other two measured the nearest minute). While this precision is likely not necessary for the average OD study, a time stamp to the nearest second may be useful, for example, in conducting a travel time study on a corridor.

\subsection{Digital Video Equipment}

The following table summarizes the features that were available on the camcorders used for this project. The three camcorder models used were 1) Panasonic PV-GS19, 2) Canon ZR-80, and 3) Canon GL2.

Table 5: Summary of Camcorder Features

\begin{tabular}{|l|c|c|c|}
\hline Feature & $\begin{array}{c}\text { Panasonic } \\
\text { PV-GS19 }\end{array}$ & Canon ZR-80 & Canon GL2 \\
\hline \hline Storage Format & MiniDV & MiniDV & MiniDV \\
\hline CCD Size & $1 / 6 "$ & $1 / 6 "$ & $3 @ 1 / 4 "$ \\
\hline Pixels (Absolute/Effective) & $680 \mathrm{k} / 340 \mathrm{k}$ & $680 \mathrm{k} / 340 \mathrm{k}$ & $410 \mathrm{k} / 380 \mathrm{k}$ \\
\hline Optical Zoom & $24 \mathrm{x}$ & $18 \mathrm{x}$ & $20 \mathrm{x}$ \\
\hline Shutter Speed & $1 / 60-1 / 8000$ & $1 / 60-1 / 2000$ & $1 / 8-1 / 15000$ \\
\hline Auto Focus & Yes & Yes & Yes \\
\hline Manual Focus & Yes & Yes & Yes \\
\hline Time Stamp to sec & Yes & No & No \\
\hline Frame-by-Frame Playback & No & No & Yes \\
\hline Cost & $\$ 349$ & $\$ 329$ & $\sim \$ 2500$ \\
\hline
\end{tabular}

Both the PV-GS19 and ZR-80 are low-end consumer camcorders, while the GL2 is a professional grade camcorder. It can be seen that, while the GL2 is much more expensive than its low-end counterparts, the only feature that is significantly different is 
the number and size of the CCDs. For license plate recording, this feature is not very important, because the quality of the color on the video will not have much (if any) effect on the clarity of the license plate digits.

\subsection{Evaluation of Digital Video Equipment}

The following field tests illustrate the issues to consider when using a camcorder for license plate data collection. These issues relate to camcorder settings (such as exposure, shutter speed, and magnification) to traffic conditions (such as vehicle speed and traffic flow rates). These tests highlight the camcorder features to look for when purchasing a digital camcorder for license plate data collection, as well as the optimal camcorder settings for a specific set of traffic conditions.

\subsubsection{Typical Roadside \& Overhead Setup}

Figures 16 and 17 illustrate the typical setup for all testing conducted with a camcorder from a roadside perspective and an overhead perspective. The following paragraphs explain the dimensions and variables in the figures in greater detail.

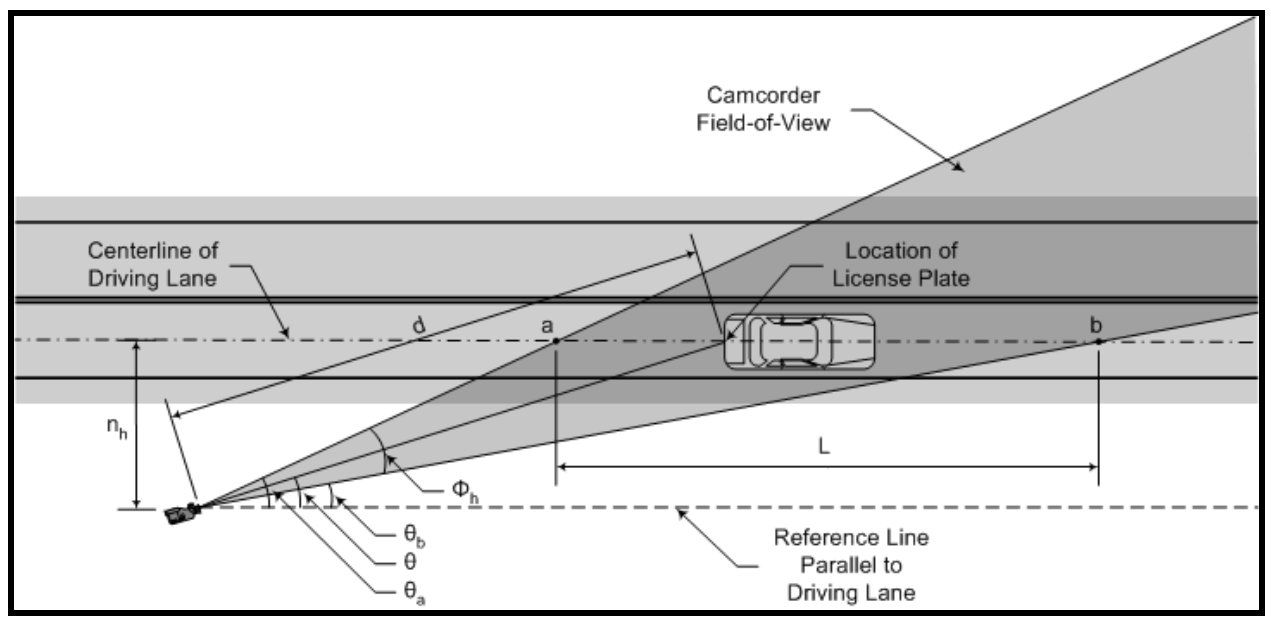

Figure 16: Plan View of Typical Camcorder Roadside Setup 


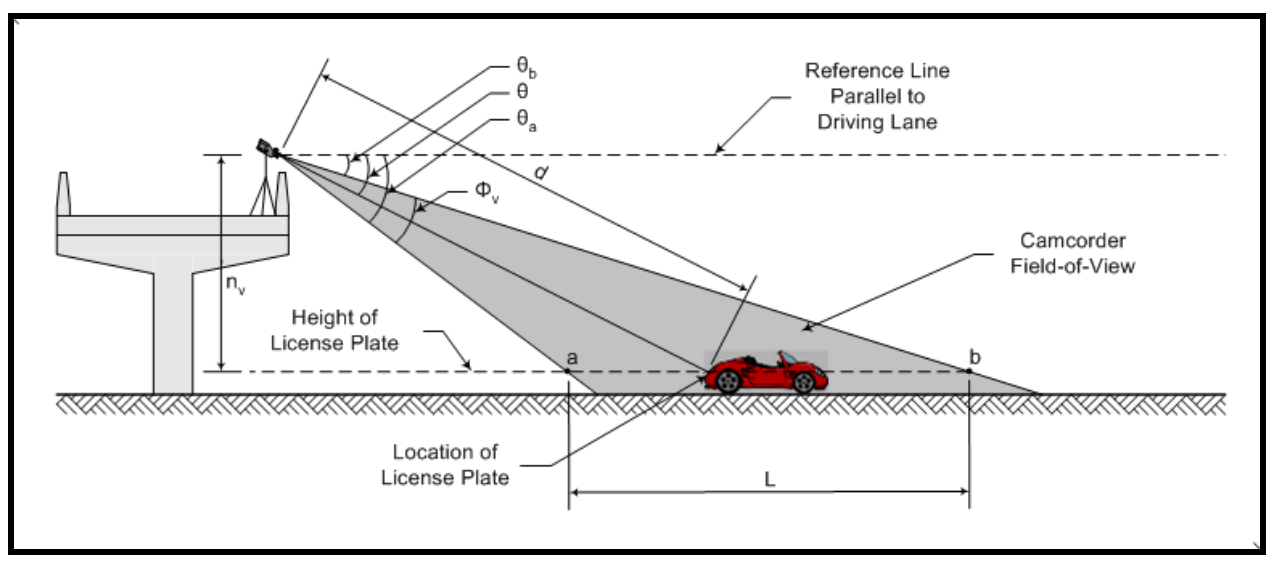

Figure 17: Elevation View of Typical Camcorder Overhead Setup

In the figures above, the subscript $h$ refers to horizontal dimensions, and the subscript $v$ refers to vertical dimensions. The distance from the lane edge at which the camcorder is set (on a tripod) is noted by $n$. This distance should be minimized, but the safety of the observer (camcorder operator) is most important. Advanced warning and/or traffic cones should be utilized to emphasize the observer's presence, especially on high speed and rural roads.

The shooting angle is the angle between the center of the field of view and some reference line. The shooting angle is denoted by $\theta$ in Figures 16 and 17 . This angle is measured from a line parallel to the centerline of the driving lane. Therefore, when $\theta=0$ degrees, the camcorder is aimed parallel to the road; and when $\theta=90$ degrees, the camcorder is aimed perpendicular to the road.

The angle-of-view is the angle between the edges of the picture and the camcorder lens, and is denoted by $\Phi$ in Figures 16 and 17. For small zooms and focal lengths, $\Phi$ is a wide angle. As the zoom and focal length increase, $\Phi$ decreases. The relationship between zoom and angle-of-view will be discussed in greater detail later. The shooting angle $\theta$ bisects the angle-of-view $\Phi$.

The left (bottom) edge of the picture on the viewfinder is the left (bottom) edge of the field-of-view. This line can be denoted as $\theta_{a}$ (in Figures 16 and 17) which is equal to $\theta+1 / 2 \Phi$. Likewise, the right (top) edge of the field-of-view, denoted as $\theta_{b}$, is equal to $\theta$ $1 / 2 \Phi$. As $\Phi$ decreases, $\theta_{a}$ and $\theta_{b}$ approach $\theta$.

For a vehicle traveling on the roadway, its license plate will appear on the viewfinder at point $a$, which is the point where the line along $\theta_{a}$ intersects with the 
centerline of the driving lane. Likewise, the license plate will disappear from the viewfinder at point $b$, which is the point at which the line along $\theta_{b}$ intersects with the centerline of the lane. The distance $L$ between points $a$ and $b$ can be found using simple trigonometry. The time the license plate remains in the field-of-view $t_{L}$ for a given $\Phi$ is a function of the speed of the vehicle $v$. Values for each of these variables assume the license plate is mounted on the center of the vehicle on a vehicle traveling down the center of the lane.

\subsubsection{Lighting Conditions}

The legibility of license plates under various lighting conditions was evaluated. All recording was conducted on the same roadway. The roadway on which the recording was done has an east-west alignment and a speed limit of $35 \mathrm{mph}$. For each lighting condition, several exposure settings were evaluated. All other camcorder settings were held constant.

Most digital camcorders have preset exposure and shutter speed settings for amateur videographers with point-and-shoot capabilities in mind. These include automatic (which optimizes exposure for a given lighting condition), sports (for fast moving objects), surf \& ski (for high-glare situations), low-light (for poorly lit rooms), and spotlight (for theatre-like conditions). This allows the videographer to avoid having to find the optimal settings manually on his or her own.

Not all camcorders allow for manual adjustment of any or all of these features. The Panasonic camcorder, however, that was used in this testing allows the user to adjust all of them manually.

Four lighting conditions were tested: overcast, sun overhead, sun behind camcorder, and sun in front of camcorder. Figure 18 illustrates the four lighting conditions evaluated. 


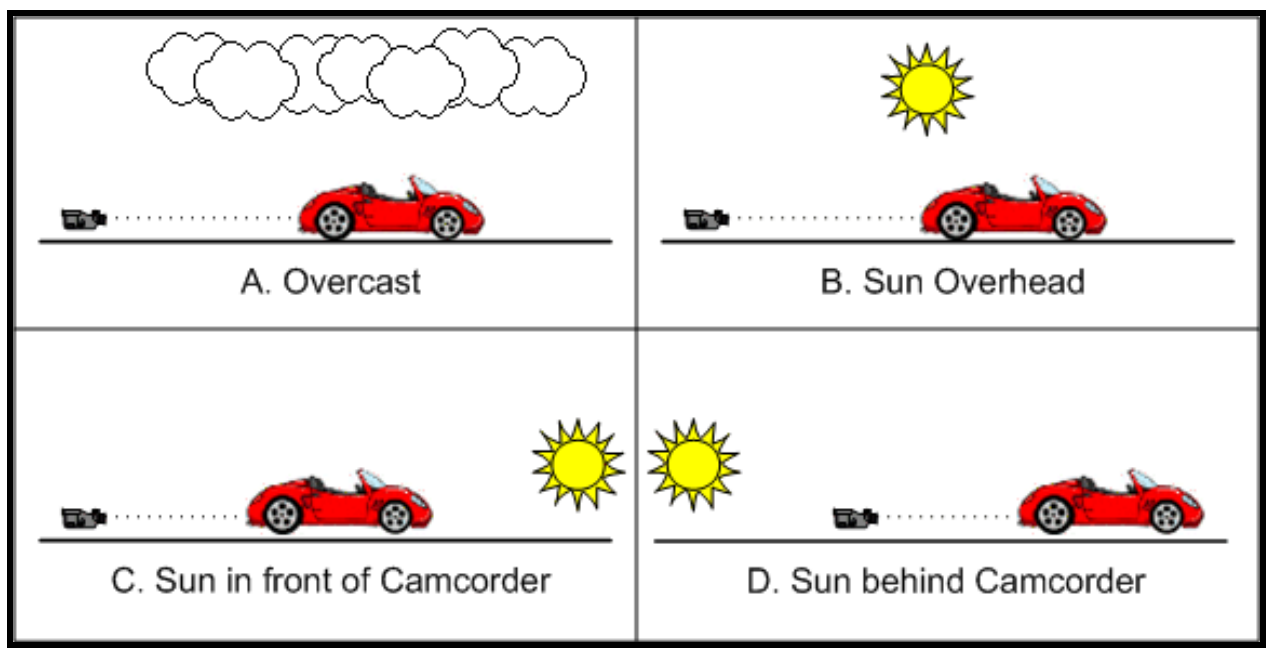

Figure 18: Lighting Conditions Encountered on the Roadside

Upon evaluation of each of the settings, it is evident that lighting is not a problem the vast majority of the time. In most conditions, the automatic exposure setting provided an adequate amount of light that was neither too bright nor too dark to see the contrast between the license plate string and the background of the license plate. There are a few circumstances worth noting that are discussed in greater detail below.

Typically, the preset exposure settings had little visual effect on the quality of the footage. The surf \& ski mode (for high-glare situations) did not seem to have much effect in sunny conditions. The automatic setting for exposure was relatively the same as the preset modes under all lighting conditions.

Under the 'sun overhead' condition, many license plates were recorded that were partially shadowed due to being inset into the vehicle. In all cases, the contrast between the sun and shadowed parts of the plate did not affect the legibility of the license plate string.

When the sun is behind the camcorder, glare (white-out of the license plate) is very rarely a problem. Glare occurs when the angle at which the license plate is mounted on the vehicle (uncontrollable) and the angle of the camcorder relative to the sun (controllable) are equal. To avoid this, the camcorder angle should not be positioned such that the sunlight hits the license plate and bounces directly towards the camcorder. This, however, cannot be controlled for every passing vehicle. However, as will be discussed later, the camcorder angle is also governed by traffic speed and 
vehicle flow so, ultimately, it is impossible to prevent glare in all circumstances for all vehicles.

When the sun was in front of the camcorder, silhouetting of the vehicle (appearing black in front of a bright background) was also generally not a problem, however, in this test, the vehicle was not directly between the sun and the camcorder. Obviously, the sun should not appear directly in the viewfinder, but this is also largely a function of the alignment of the roadway relative to the location of the sunrise and sunset, which varies by time of day and season.

It was not determined how much daylight had to be present in order for lighting not to be a problem because the amount of daylight at a certain time of day changes with the calendar. Generally, OD studies should not be conducted before sunrise or after sunset for the safety of the observers. If possible then, studies using video should be conducted during the season in which the start and end times occur during daylight hours, preferably when the sun is already above the horizon.

\subsubsection{Focus}

Most digital camcorders have automatic focus. Some, however, include manual focus as a feature. The camcorders used in this project all have automatic focus. In most cases, automatic focus was not a problem. There are, however, a few circumstances to avoid.

First, when choosing a location to set up the camcorder, select a location that does not have any traffic signs, posts, poles, or other obstruction downstream. The obstruction-free distance depends on the camcorder shooting angle and the distance at which the camcorder is set up from lane edge. If a post or other object appears in the viewfinder when shooting a vehicle beyond it, the camcorder may focus on the obstruction in the foreground image and not the vehicle.

In addition, avoid shooting in an area where pedestrians can walk into the fieldof-view, for example, shooting across a sidewalk parallel to the roadway. This is especially true when the shooting angle is less than 15 degrees, because the pedestrians will remain in the field-of-view for a significant amount of time. If it cannot be avoided, direct pedestrians around the field-of-view so they do not shift the focus from the vehicles. 


\subsubsection{Obstruction \& Minimum Shooting Angles}

When recording the license plate from a single vehicle traveling on a roadway, the theoretical optimal horizontal and vertical shooting angle is zero. This, however, means the camcorder is set up in the center of the traffic lane at a height of approximately 3 feet (the height of the average license plate). In this circumstance, the license plate would appear in the center of the camcorder's field-of-view and remain stationary while the license plate characters shrink as the vehicle travels farther and farther away. Obviously, however, the camcorder can never be set up in the traffic lane.

The second-best location for camcorder set up off the roadway is either centered above the traffic lane (e.g., on an overpass) or on the side of the road. If the camcorder is set on an overpass, the horizontal angle is zero, but the vertical angle is not. In addition, the vertical distance $n_{v}$ from the camcorder to the license plate cannot be less than $\sim 20$ feet (the height of the overpass deck plus tripod height minus the height of the license plate). Conversely, on the side of the road, the vertical angle is close to zero, but the horizontal angle is not. In addition, the horizontal distance $n_{h}$ of the camcorder on the side of the road cannot be less than $\sim 7$ feet (half the width of the traffic lane plus one foot for the tripod). While any shooting angle can be achieved regardless of the distances $n_{v}$ or $n_{h}$, the zoom capabilities of the camcorder will also limit the minimum shooting angle. This will be discussed in the next section.

From a vantage point on the roadside, a vehicle following another will eventually (some distance downstream) block the view of the license plate of the first vehicle. Given this following distance and the distance $n_{h}$, an angle can be calculated. At this angle, the license plate of the first vehicle will come into view just as the right-front corner of the following vehicle enters the field-of-view. Likewise, in the overhead setup, the top of the following vehicle enters the field-of-view just as the license plate of the first vehicle enters the field-of-view. Figures 19 and 20 illustrate this. This obstruction angle is a function of the distance between the rear of the first vehicle and the front of the following vehicle (which usually increases as speed increases), the height of the following vehicle (for overhead setup), the location of the license plate on the first vehicle, and the lateral location of both vehicles in the traffic lane. 


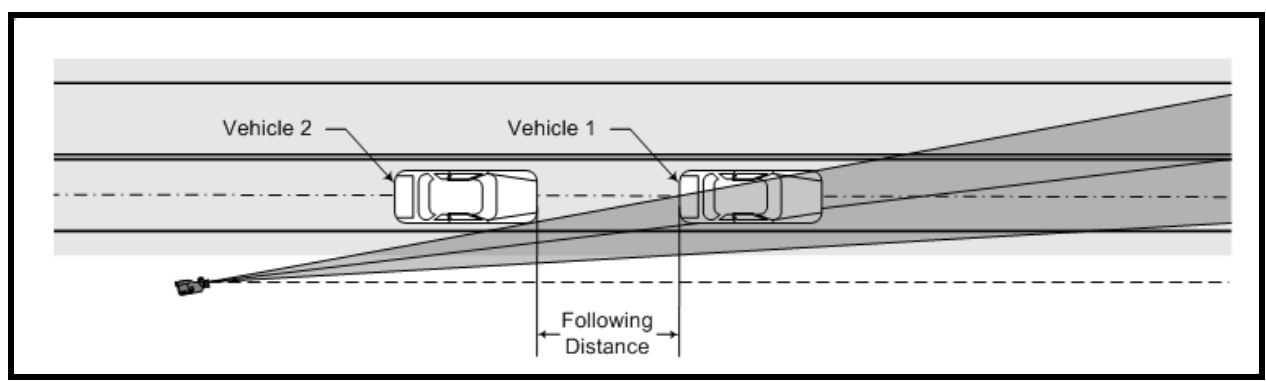

Figure 19: Following-Vehicle Obstruction from the Roadside Perspective

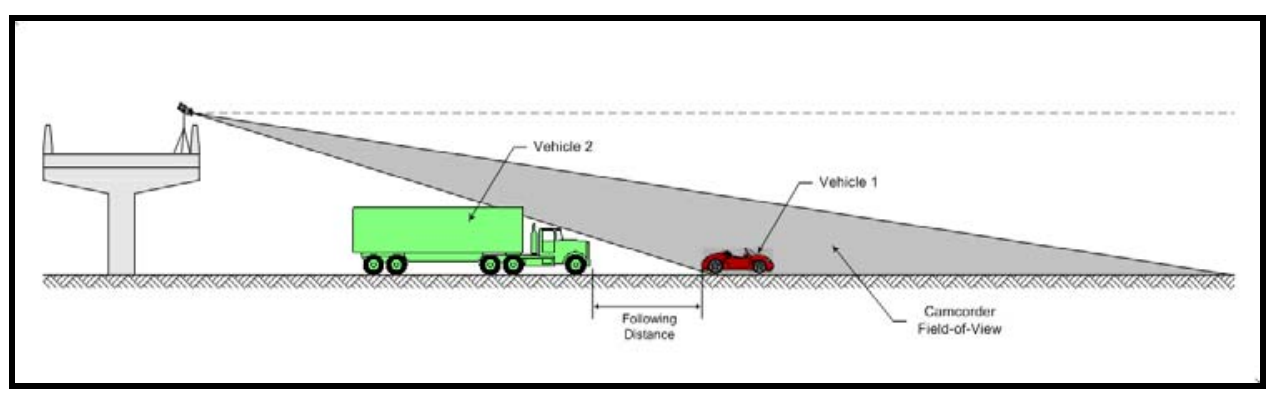

Figure 20: Following-Vehicle Obstruction from the Overhead Perspective

Figures 21 and 22 illustrate the obstruction angle for three different following times. Following time is similar to headway, except following time is measured from the rear of one vehicle to the front of the next, and headway is measured between the fronts of subsequent vehicles. For high speeds, the difference between following time and headway is small. Following times are assumed to be constant for all speeds, but the corresponding following distance increases as speed increases. As the following distance increases, the obstruction angle decreases. Therefore, the obstruction angle decreases as speed increases. 


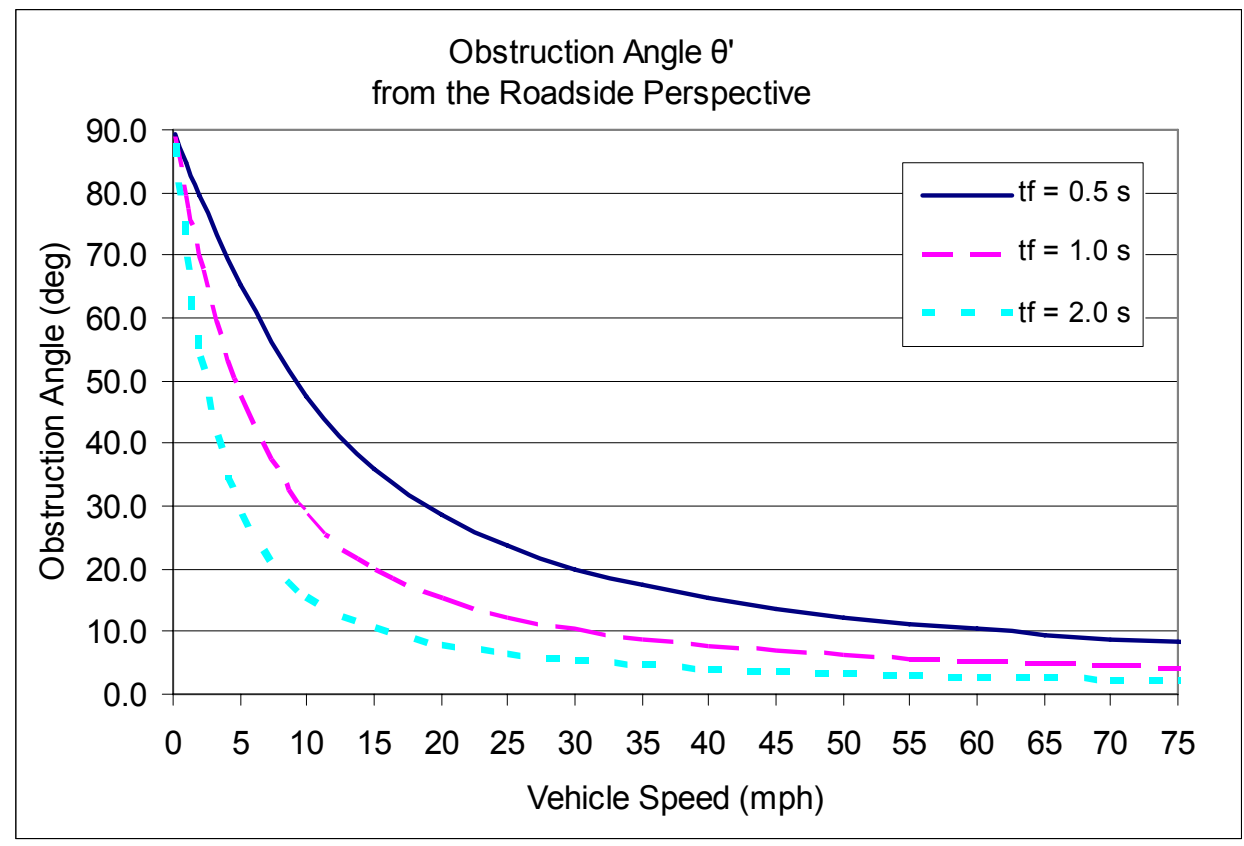

Figure 21: Obstruction Angle from Roadside Perspective

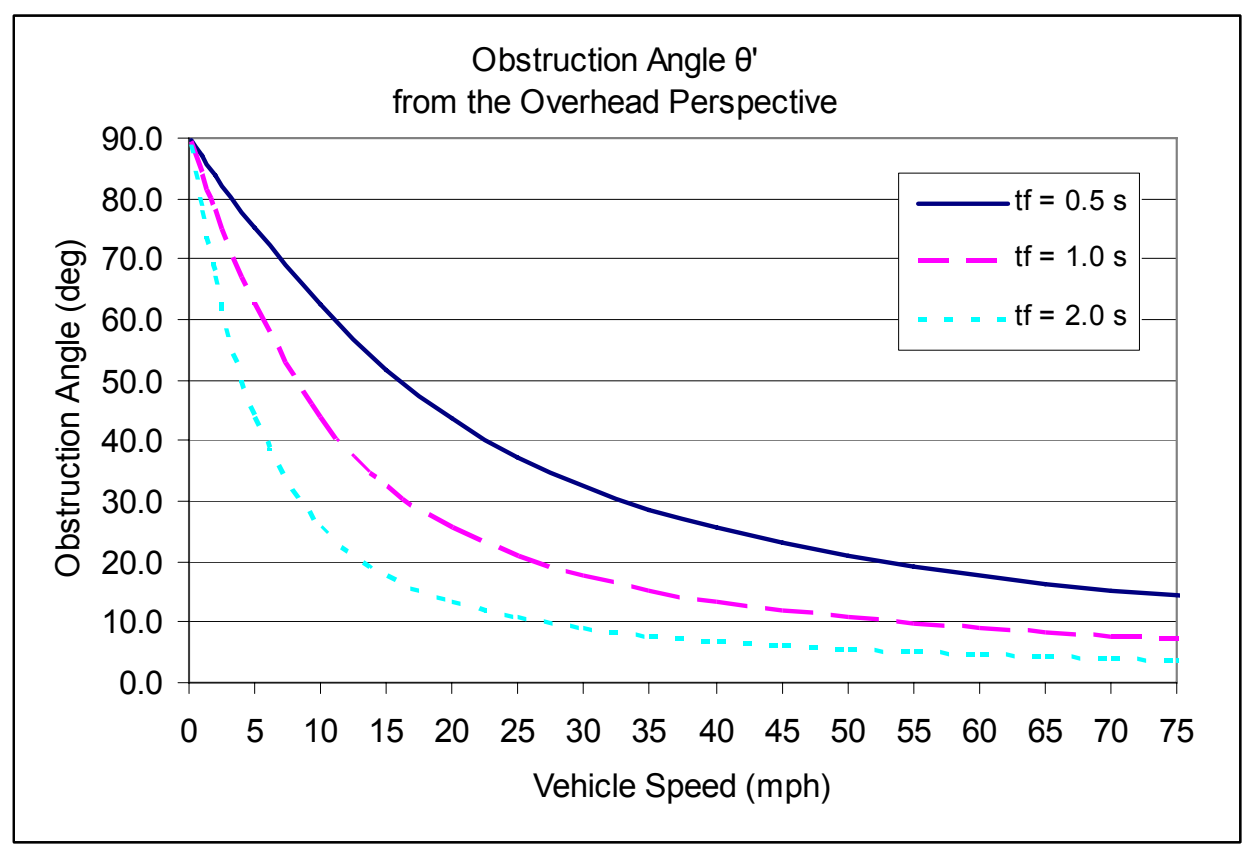

Figure 22: Obstruction Angle from Overhead Perspective

In low, free-flow traffic conditions, most vehicles maintain a following distance of at least 2 seconds. In this case, the angles corresponding to the 2-second following time should be used. In high flow, urban conditions (especially downstream from traffic 
signals) or congested conditions, a greater percentage of vehicles have a following time of less than 2 seconds. In these situations, the angles corresponding to the $1 / 2$ or 1 second following times should be used. Regardless of the angle chosen, a small percentage of vehicles will still be blocked from the camcorder's field-of-view.

When setting up a camcorder, the minimum shooting angle will be equal to the obstruction angle $\theta$ ' plus one-half the angle-of-view $\Phi$. The angle-of-view will be discussed in the next section.

When using camcorders, there may be some situations in which a curve in the road may be used to the advantage of the camcorder. The goal of setting up on a curve is that it may reduce or eliminate the shooting angle of the camcorder. This is advantageous because it reduces the speed at which the license plate traverses the screen and increases the amount of time the license plate remains present on the screen, which is beneficial for manual transcription. Figure 23 illustrates an example of this.

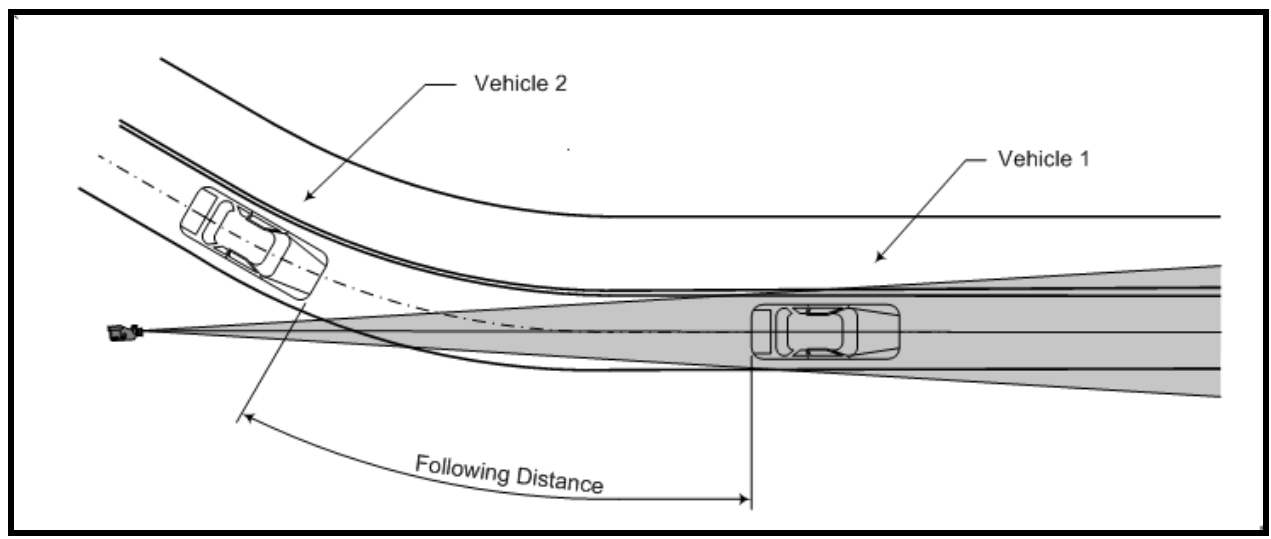

Figure 23: Setup on Curves in the Road

Depending on the radius of the curve and the distance between vehicles traveling on the road (which increases with speed), the shooting angle may or may not be reduced to zero. However, depending on the circumstances, that reduction may or may not be advantageous.

\subsubsection{Magnification and Angle-of-View Variations by Camcorder Model}

Camcorder models are manufactured with a wide variety of lenses, which affects the maximum optical zoom of the camcorder. An optical zoom works by physically 
adjusting the distance (called the focal length) between the lens and the CCD. Again, the CCD is the digital version of film. Figure 24 is a simple illustration of a lens.

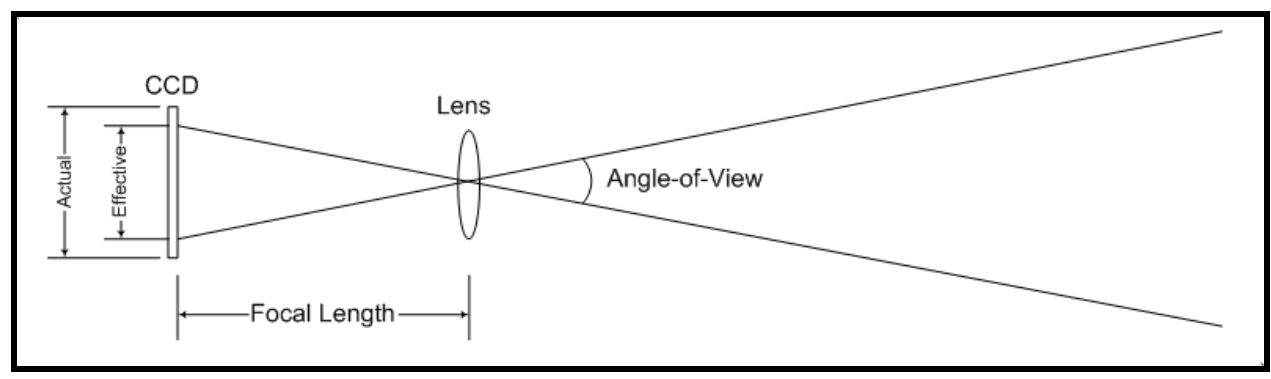

Figure 24: Dimensions of Typical Camcorder Lens

An optical zoom of 10x means that an object will be magnified ten times as much as the same object at a zoom of $1 \mathrm{x}$. The zoom is directly related to the range of focal length of the lens. For example, if the focal length of the lens ranges from $2.1 \mathrm{~mm}$ to $50.4 \mathrm{~mm}$, the lens has a maximum optical zoom of $24 x(50.4 / 2.1)$. This means that an object can be magnified up to 24 times. A focal length of $4.2 \mathrm{~mm}$ on that same lens corresponds to a zoom of $2 \mathrm{x}$, a focal length of $8.4 \mathrm{~mm}$ corresponds to $4 \mathrm{x}$, etc.

Unfortunately, a zoom of $A x$ on one camcorder will magnify an object more or less than a zoom of $A x$ on another camcorder. This is because the range of focal lengths of the lens on the second camcorder can, and often will, be different. For example, the lens mentioned previously had a focal length range of $2.1 \mathrm{~mm}-50.4 \mathrm{~mm}$ for a maximum zoom of $24 x(50.4 / 2.1)$. Another camcorder might have a focal length of $2.8 \mathrm{~mm}-56.0 \mathrm{~mm}$ for a maximum zoom of $20 x(56.0 / 2.8)$. If both of these camcorders are set up side by side and focus on the same object at $1 \mathrm{x}$ (or any other magnification), the object will always appear smaller through the first camcorder. The reason for this is the angle-of-view.

The angle-of-view is directly related to the focal length. As the focal length increases (and the zoom increases), the angle-of-view decreases. This enables the magnified object to appear on the viewfinder or screen (which stays the same size, of course). Theoretically then, at a zoom of $1 x$, the object shot by the first camcorder will appear $75 \%(2.1 / 2.8)$ as large as the same object shot by the second camcorder. It should be noted, that there is more than one angle-of-view, as illustrated in Figure 25. 
However, the ratios between the angles-of-view remain constant as the focal length changes.

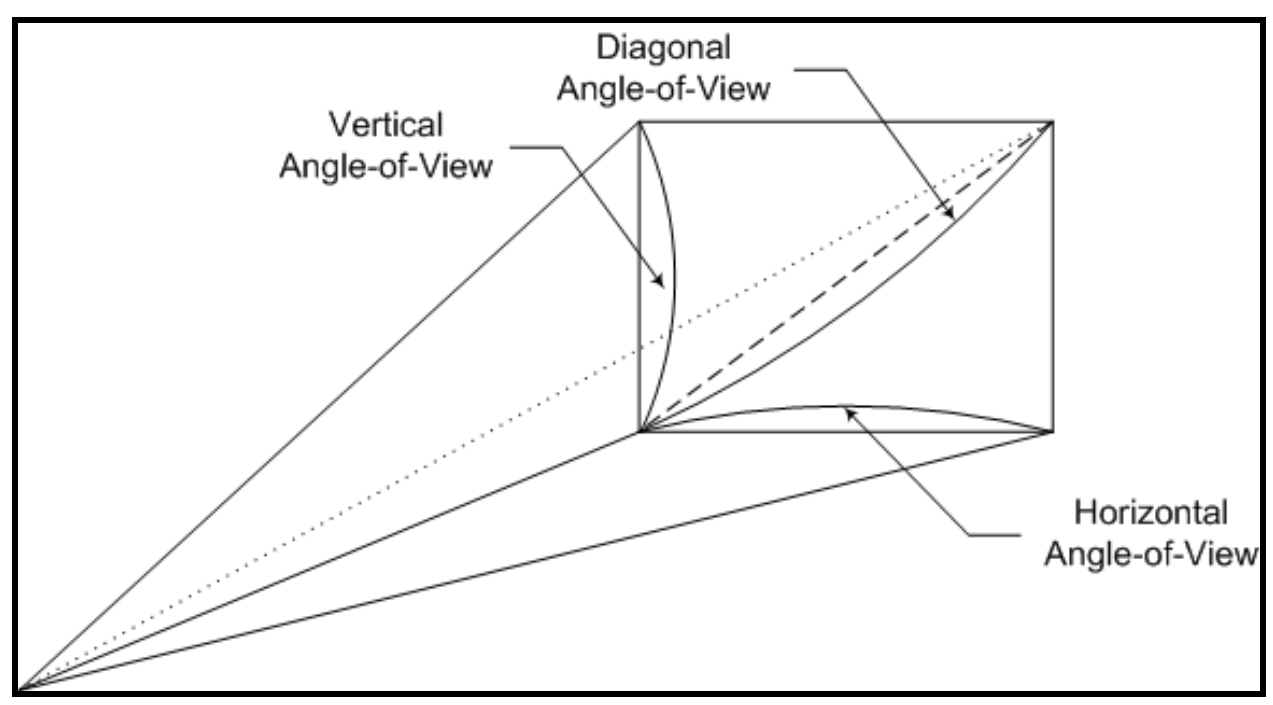

Figure 25: Illustration of Angles-of-View

Further complicating matters is that, for any single focal length, the angle-of-view depends on the size of the CCD. CCDs come in many sizes, between 1 " for professional-grade models down to $1 / 6$ " or $1 / 8$ " for consumer-grade models. Larger CCDs are necessary if the video will be viewed on very large screens.

The CCD is measured diagonally, much like television screens. Standard $35 \mathrm{~mm}$ film, for example, actually measures $43.3 \mathrm{~mm}$ diagonally. It would be assumed then, that a 1" CCD is $25.4 \mathrm{~mm}$ by simple unit conversion. Unfortunately, the lack of standards complicates this even more, because 1" is just a nominal measurement. The actual diagonal measurement of any size CCD is roughly (but not exactly) $2 / 3$ of the nominal measurement. A 1" CCD effectively measures $16.0 \mathrm{~mm}$ diagonally.

Now that the effective diagonal length of the CCD is known approximately, an actual angle-of-view can be calculated. Figure 26 illustrates the relationship between focal length, CCD size, and angle-of-view. Table 6 shows the actual angle-of-view for the PV-GS19 and ZR-80 camcorders. By looking at the predicted versus the actual values of the angles-of-view, a significant error still exists in that the predicted value is consistently higher than the actual value. This may be because CCDs of the same 
nominal size may actually be significantly different (from model to model), or the actual display of the zoom of the camcorder is not accurate in the viewfinder.

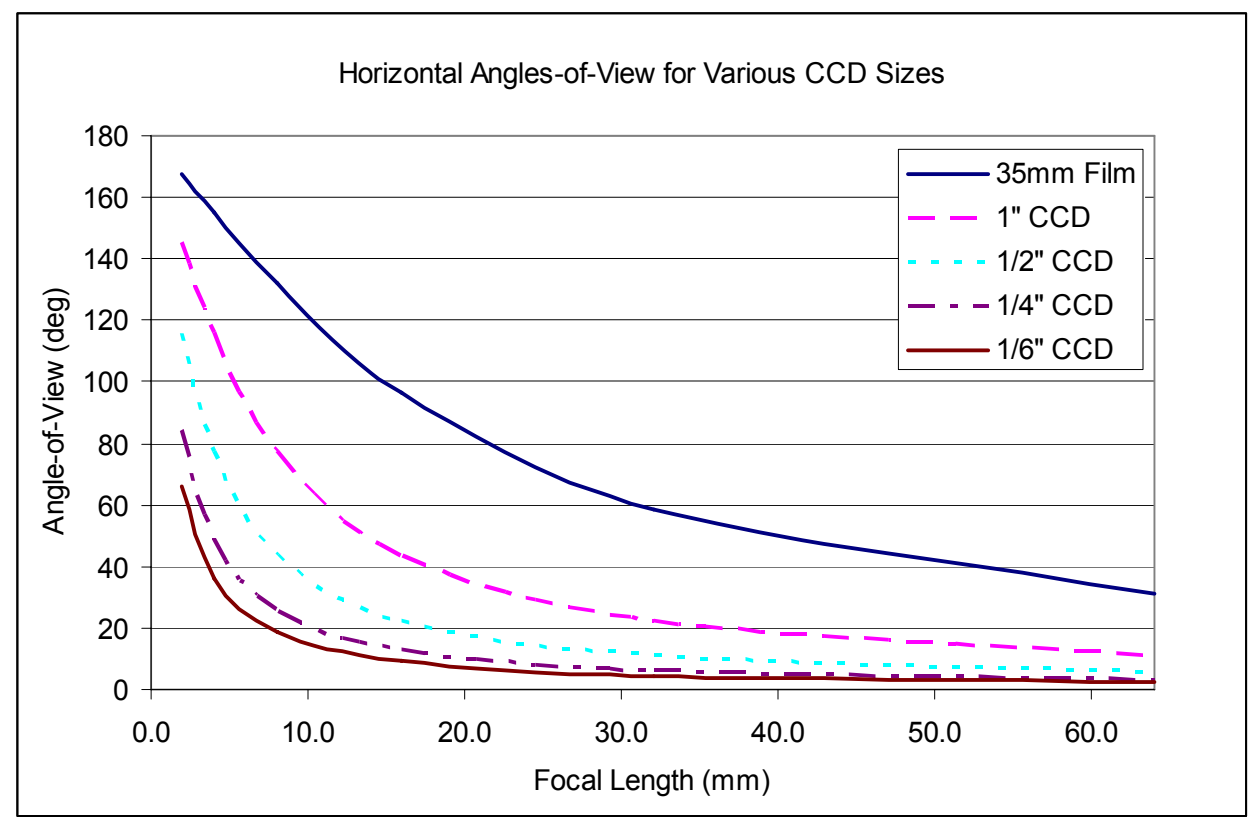

Figure 26: Relationship between Focal Length \& Angle-of-View by CCD Size

Table 6: Predicted vs. Actual Angles-of-View

\begin{tabular}{|c|c|c|c|c|}
\hline \multicolumn{5}{|c|}{ Panasonic PV-GS19 (1/6" CCD) } \\
\hline Zoom & FL & Predicted & Actual & $\%$ Diff \\
\hline \hline $1 \mathrm{x}$ & 2.1 & 64 & 45 & $29.7 \%$ \\
\hline $2 \mathrm{x}$ & 4.2 & 34 & 30 & $11.8 \%$ \\
\hline $4 \mathrm{x}$ & 8.4 & 18 & 13 & $27.8 \%$ \\
\hline $8 \mathrm{x}$ & 16.8 & 8.8 & 6.9 & $21.6 \%$ \\
\hline $16 \mathrm{x}$ & 33.6 & 4.4 & 3.4 & $22.7 \%$ \\
\hline $24 \mathrm{x}$ & 50.4 & 3.0 & 2.3 & $23.3 \%$ \\
\hline Canon ZR-80 (1/6" CCD) \\
\hline Zoom & $\mathrm{FL}$ & Predicted & Actual & $\%$ Diff \\
\hline \hline 1x & 2.8 & 50 & 33 & $34.0 \%$ \\
\hline $18 \mathrm{x}$ & 50.4 & 3.0 & 2.2 & $26.7 \%$ \\
\hline
\end{tabular}

All of this boils down to the fact that, for shooting license plates at an angle of $\theta$ at a distance of $n_{h}$ feet from the lane edge, an optimal zoom cannot be specified to ensure that the license plate characters will not be too small or too large for transcription during playback. As a result, the magnification of the license plate will have to be 
roughly specified relative to the size of the viewfinder on the camcorder. This will be discussed in the next section.

\subsubsection{Magnification}

Because magnification differs among camcorder models, the amount of magnification required for any setup location will be defined by the size of the license plate in the center of the field-of-view relative to the field-of-view. The minimum and maximum magnification will be discussed below in addition to the magnification limitations on the minimum shooting angle.

For any camcorder set up on the roadside or overhead, magnification will likely be necessary in order for the license plate characters to be large enough to be legible during playback. On many camcorders, the quality of the picture as seen on the viewfinder is generally lower than as it appears during playback on a television. Therefore, it may be difficult to know in the field if the magnification is enough for the license plate characters to be legible. While there is some leeway, a good rule of thumb is to magnify until the rear of the vehicle fills the width of the field-of-view. The ratio of the width of the license plate $w$ to the width of the viewfinder $W$ is approximately 0.15 . For large shooting angles (above $30^{\circ}$ ), this ratio may be reduced slightly to adjust for the skewness of the shooting angle relative to the rear of the vehicle. Figure 27 illustrates this magnification ratio. 


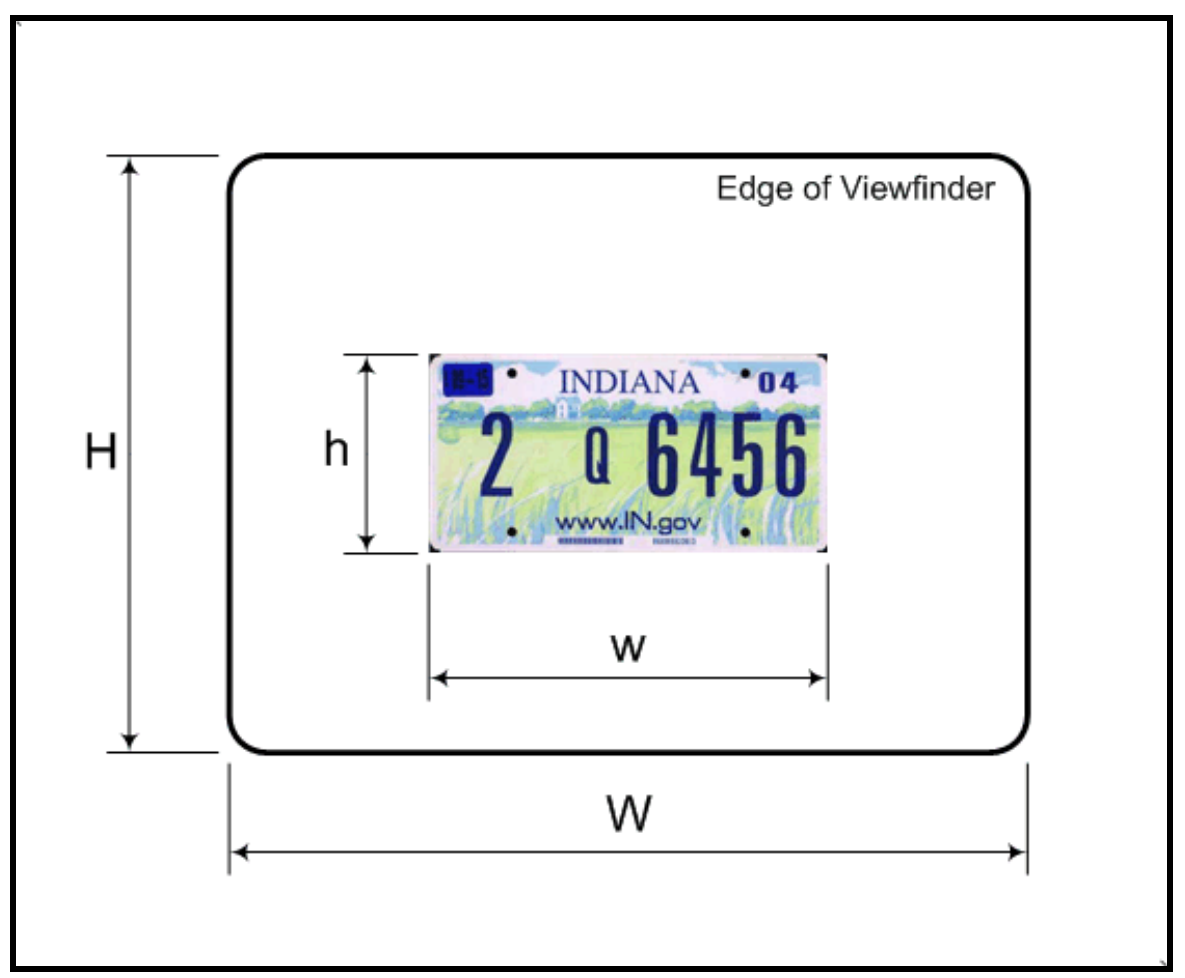

Figure 27: Required Magnification of License Plates

While the minimum magnification is necessary for legibility, magnification much beyond 0.15 should be avoided. This is because, when magnified too much, license plates that are mounted at different heights (especially on trucks) may not appear within the field-of-view at all.

Finally, when the camcorder shooting angle is set, the optical magnification capabilities of the camcorder may not allow the minimum ratio to be achieved. In addition, digital magnification should never be used for a license plate survey. In this case, the distance $n$ from the camcorder to the traffic lane should be reduced. This however, is not possible from an overhead perspective, and may be limited from a roadside perspective due to safety issues. If the camcorder cannot be moved, then the shooting angle will have to be increased until the magnification ratio can be achieved.

\subsubsection{Optimal Shutter Speed}

The shutter speed is a very important aspect in setting up the camcorder. A higher shutter speed can record moving objects more clearly. At a low shutter speed, the object will appear blurry or streak across the field-of-view. The optimal shutter speed 
depends upon the speed of the vehicle perpendicular to the line-of-sight of the camcorder. Figure 28 illustrates this.

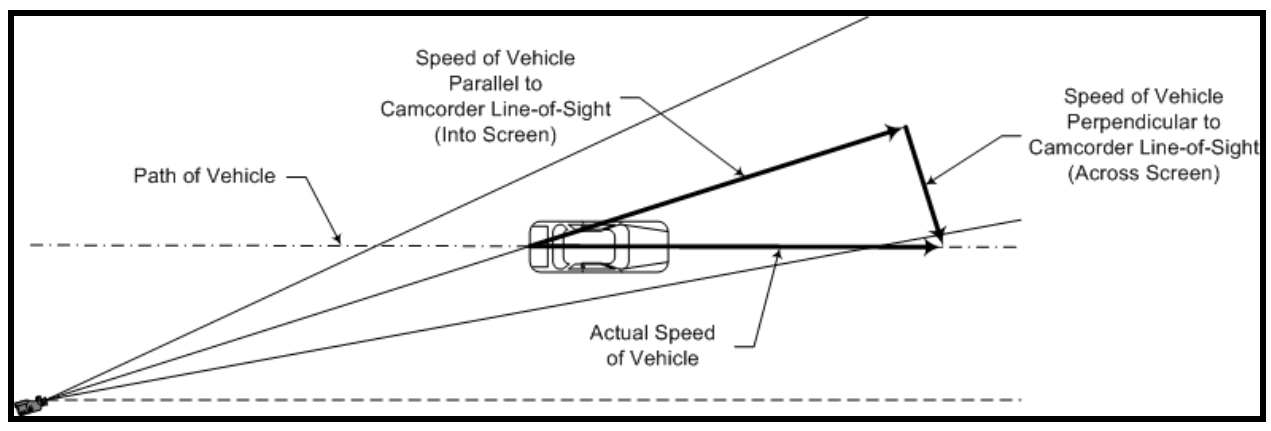

Figure 28: Vehicle Speed Perpendicular to the Camcorder Line-of-Sight

If the camcorder were set up in the center of the traffic lane at the height of the license plate (shooting angle equals $0^{\circ}$ ), the vehicle would not move laterally across the screen (although it would get smaller as it moved farther away). In this case, a very low shutter speed would be acceptable. However, if the shooting angle of the camcorder was $90^{\circ}$, the vehicle's speed would appear laterally across the screen but not into the screen. In this case, a high shutter speed is required to prevent streaking of the picture. Because the shutter speed is sensitive to the lateral movement (or perpendicular movement) of the vehicle, the perpendicular vehicle speed needs to be calculated in order to find the optimal shutter speed. Table 7 illustrates the perpendicular vehicle speed as a function of the actual vehicle speed and shooting angle.

Table 7: Perpendicular Vehicle Speed (mph)

\begin{tabular}{|c|c|c|c|c|c|c|c|}
\hline & \multicolumn{6}{|c|}{ Shooting Angle (Line-of-Sight Angle), degrees } \\
\hline & & 0 & 5 & 15 & 30 & 45 & 90 \\
\hline \multirow{8}{*}{ 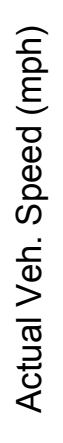 } & 5 & 0.0 & 0.4 & 1.3 & 2.5 & 3.54 & 5.00 \\
\hline & 15 & 0.0 & 1.3 & 3.9 & 7.5 & 10.6 & 15.0 \\
\hline & 25 & 0.0 & 2.2 & 6.5 & 12.5 & 17.7 & 25.0 \\
\hline & 35 & 0.0 & 3.1 & 9.1 & 17.5 & 24.7 & 35.0 \\
\hline & 45 & 0.0 & 3.9 & 11.6 & 22.5 & 31.8 & 45.0 \\
\hline & 55 & 0.0 & 4.8 & 14.2 & 27.5 & 38.9 & 55.0 \\
\hline & 65 & 0.0 & 5.7 & 16.8 & 32.5 & 46.0 & 65.0 \\
\hline & 75 & 0.0 & 6.5 & 19.4 & 37.5 & 53.0 & 75.0 \\
\hline
\end{tabular}


Using the appropriate perpendicular vehicle speed, Figure 29 provides the optimal shutter speed camcorder setting. These shutter speeds were determined by manually adjusting the shooting angles and shutter speeds until the characters of recorded license plates appeared clearly during playback.

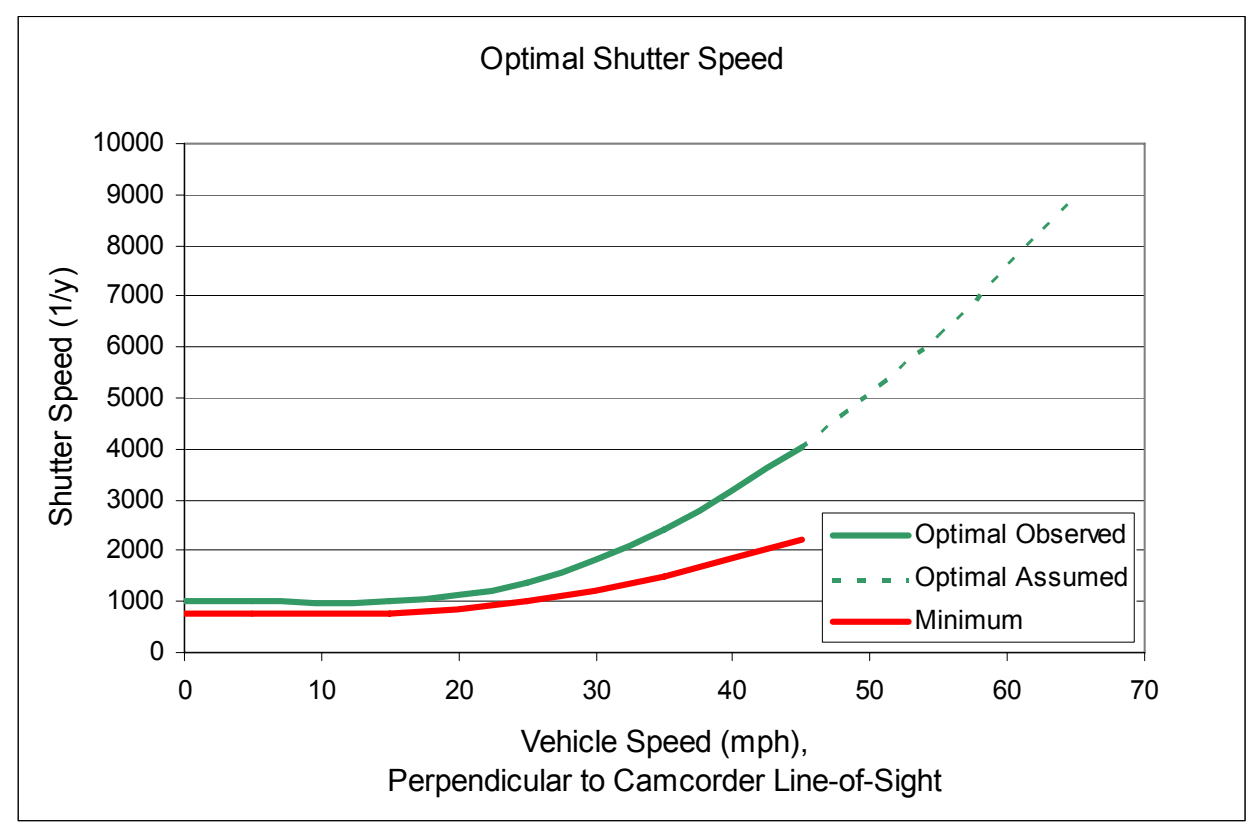

Figure 29: Optimal Shutter Speed

\subsubsection{Left Lane Obstruction}

Generally, it is not desirable to use one camcorder to record multiple lanes of traffic, especially on high volume roads. The reason for this is because vehicles in the lane closest to the camcorder will block the view of vehicles in the far lanes. This percentage of the time the far lane is blocked depends upon the amount of traffic flow and the shooting angle of the camcorder. However, for certain roadway conditions, such as undivided multilane roads, one camcorder will have to record both lanes because it is not possible to shoot the far lane unobstructed from either side of the road. Figure 30 illustrates the left-lane obstruction issue. 


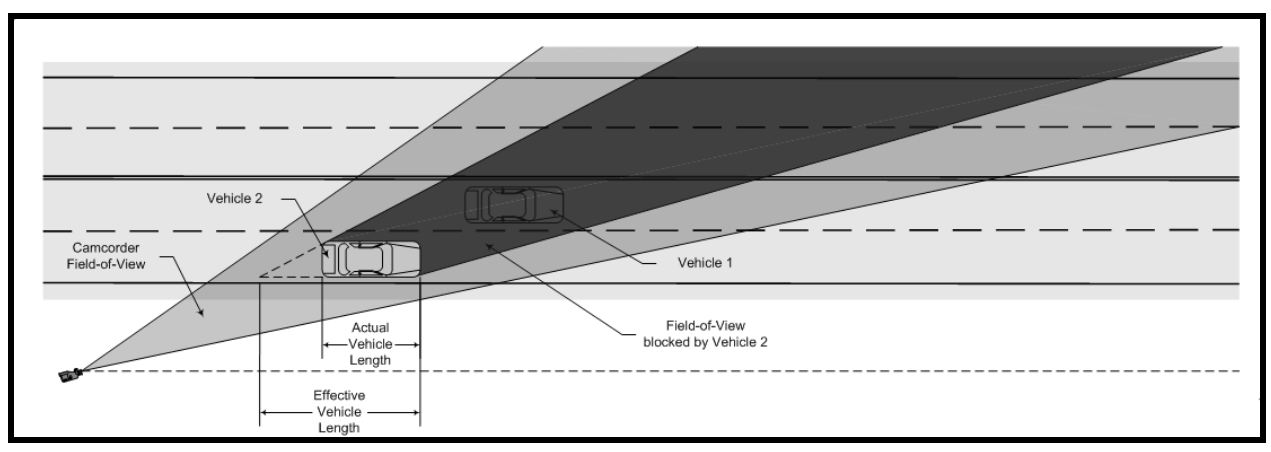

Figure 30: Obstruction of License Plates in Left Lane

The percentage of the time that the left lane is obstructed increases as vehicle speed decreases and camcorder shooting angle decreases. The graphs in Figure 31 illustrate these obstruction factors for four different vehicle speeds.
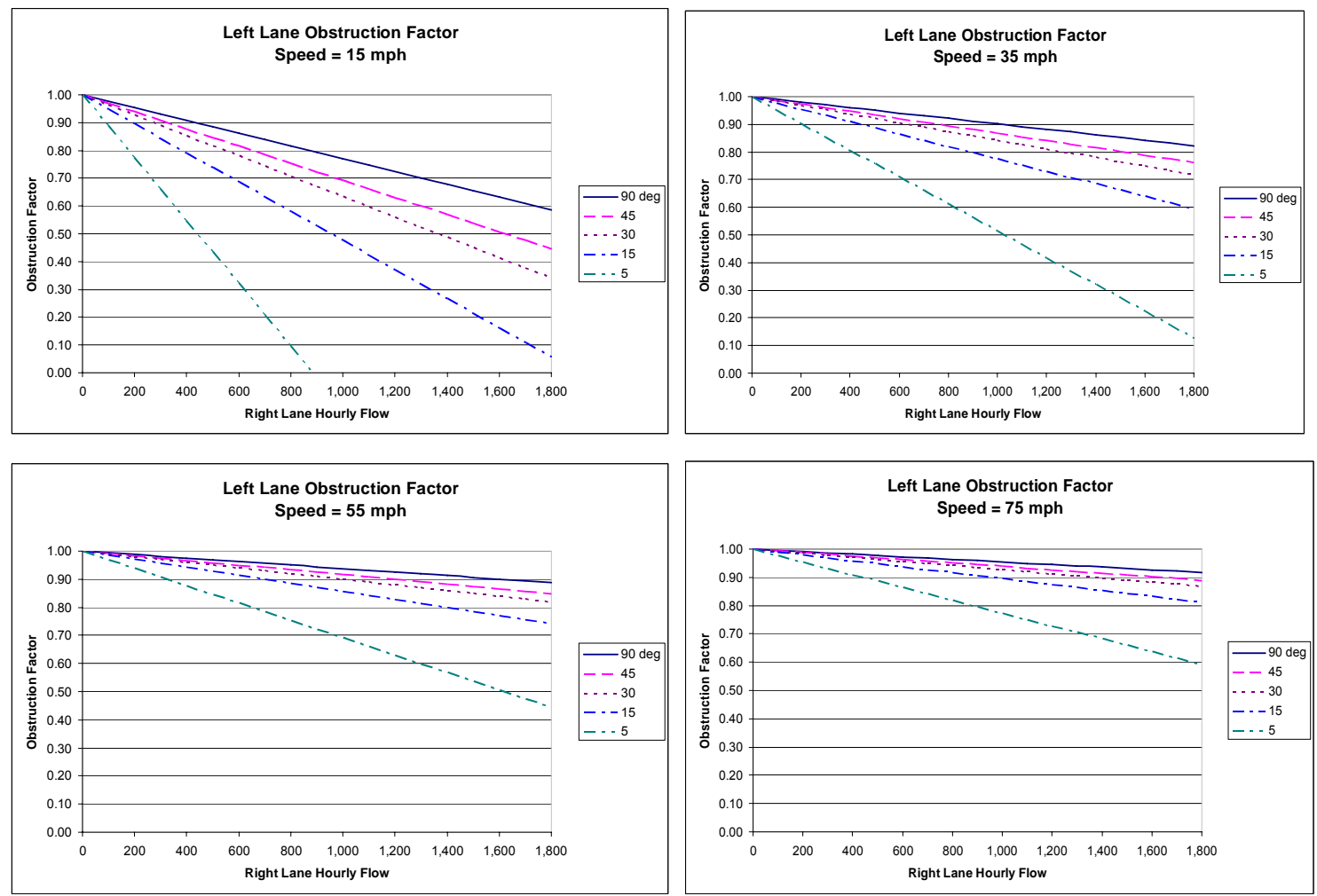

Figure 31: Left Lane License Plate Obstruction Factor

\subsection{Photography Equipment}

Photography equipment was not evaluated as part of this project. At this time, there are few, if any, manufacturers that manufacture cameras that have the features 
and capabilities required for the purposes of conducting short-term license plate data collection at temporary roadside locations. However, almost all of the issues related to using video camcorders also apply to this method. 


\section{CHAPTER 6 - DETERMINING LICENSE PLATE DATA COLLECTION METHOD}

In order to evaluate which data collection methods are suitable for a specific set of roadway characteristics and traffic conditions, license plate data was collected in the field for both the license plate matching technique (which requires that 4 characters of the license plate be recorded) and the license plate follow-up survey technique (which requires the full string and state-of-issuance so the address of the vehicle owner can be obtained through the appropriate DMV).

The process of recording a license plate string can be broken into two distinct parts: 1) identifying the string, and 2) recording the string. Section 6.3 evaluates the first step, while Section 6.4 discusses the second step of that process using the technology and equipment discussed in Chapter 5.

Before evaluating the various methods for license plate data collection, however, Section 6.1 defines the rules for 4-character and full string license plate data collection, and Section 6.2 illustrates and discusses the current license plate variations used in Indiana and surrounding states that may be encountered when conducting a license plate survey.

\subsection{License Plate Data Collection Rules}

Because there are so many variations in the syntax and size of license plate strings, several rules need to be created in order to ensure that the appropriate data is being collected in the field. The following sections discuss some important issues for the license plate matching and license plate follow-up survey techniques.

\subsubsection{License Plate Matching Technique}

In this technique, license plates are matched between observation stations. No information is collected from the driver, either on the roadside or through a follow-up 
survey. Therefore, the only data that is required from each observation station is some unique identifier for the vehicle. Recording four characters from the license plate will virtually eliminate the possibility of a spurious match. This was discussed in Chapter 2. The last four characters (not the first four) should be recorded. This is especially necessary in Indiana, because the state uses county identifiers as the first two digits on standard passenger vehicle license plates. Also, both letters and numbers should be recorded. In the case that characters are stacked vertically, the top number should be recorded first. Examples of various license plates will be shown in the Section 6.2 to illustrate these rules.

\subsubsection{License Plate Follow-Up Survey Technique}

Like the matching technique, there is no interaction with the driver during the observation of the vehicle. Instead, trip information is obtained through a mailed followup survey. In order to mail the vehicle owner a survey, the full license plate has to be recorded so the owner's address can be obtained from the state motor vehicle department. In order to contact the appropriate motor vehicle department, the state must be recorded from the license plate. Therefore, all of the characters that appear across the center of the license plate should be recorded. Other information that appears on the license plate, such as vehicle type, is not necessary because the serial number of the license plate is unique to that vehicle. For both techniques, the license plates should be recorded from the rear of the vehicle. This is because Indiana issues one license plate which is placed in the rear.

In many studies that utilize this technique, only the states that are geographically adjacent to the state in which the OD study is being conducted are contacted for owner's addresses. In Indiana, these states include Michigan, Ohio, Kentucky, and Illinois. Depending on the location of the OD study within the state, and the amount and type of out-of-state vehicles using the roadways within the study area, the adjacent states may be excluded (or other states included). If there are many out-of-state license plates recorded, not sending a survey may bias the results of the study. The states that will be included should be contacted prior to the OD study. 


\subsection{License Plate Variations}

License plates come in many different colors, fonts, and syntaxes. Generally, each of the 50 US states has a standard style for passenger vehicles. However, there are often special recognition plates and vanity plates that have different variations. Most states have additional variations for commercial vehicles and government vehicles (state, municipal, university, etc.). Fortunately, the dimensions of license plates do not vary from state to state, but smaller license plates are often used for motorcycles and small trailers.

While all states require license plates to appear on the rear of vehicles, some vehicles do not have them at all (missing or temporary) while others have plates that are damaged, dirty, blocked, or covered (by tinted plastic covers or frames).

The following paragraphs illustrate the current style of license plates issued in Indiana, as well as some of the different variations found in other states. In addition, some special cases are illustrated to highlight some of the potential problems that may be encountered in the field when conducting license plate data collection.

\subsubsection{Indiana Passenger Vehicle License Plates}

The standard passenger vehicle license plate issued by the Indiana Bureau of Motor Vehicles (BMV) is illustrated in Figure 32. The standard license plate has a one or two-digit county identifier, followed by one letter, and finally, one, two, three, or four numbers. There are currently two variations of this plate being used. The string on the first plate shown has no spaces and bold characters which are all the same height. This variation is being phased out and replaced by the second license plate shown. The string on the second plate has the same syntax, however, the characters are less bold, the letter is smaller in height, and spaces appear before and after the letter. The information next to each license plate displays the color, syntax, and information that should be recorded under the 4-character and full string rules. 


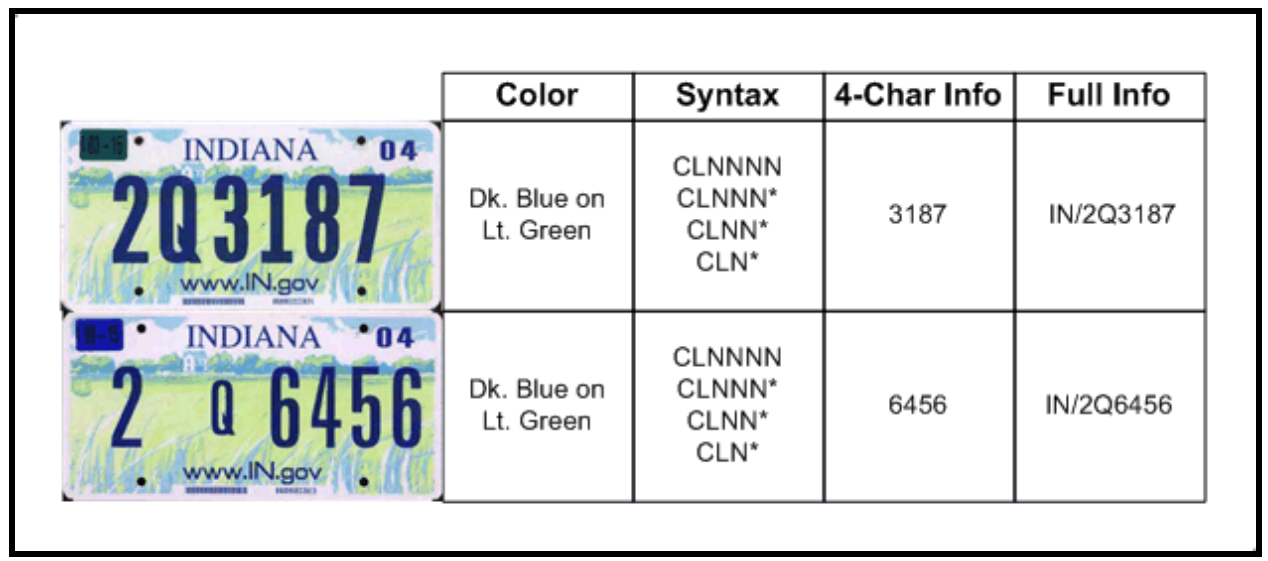

Figure 32: Indiana Passenger Vehicle Standard License Plates Source of LP Images: Nicholson, 2005, *Syntax not shown in figure

In addition to the standard license plates for passenger vehicles, there are many special license plates that recognize other various groups. According to the Indiana Bureau of Motor Vehicles website, there are currently special recognition plates for 23 colleges and universities, and 34 for other organizations ranging from military, occupational, and other non-profit groups. Figure 33 illustrates a few of these license plates.

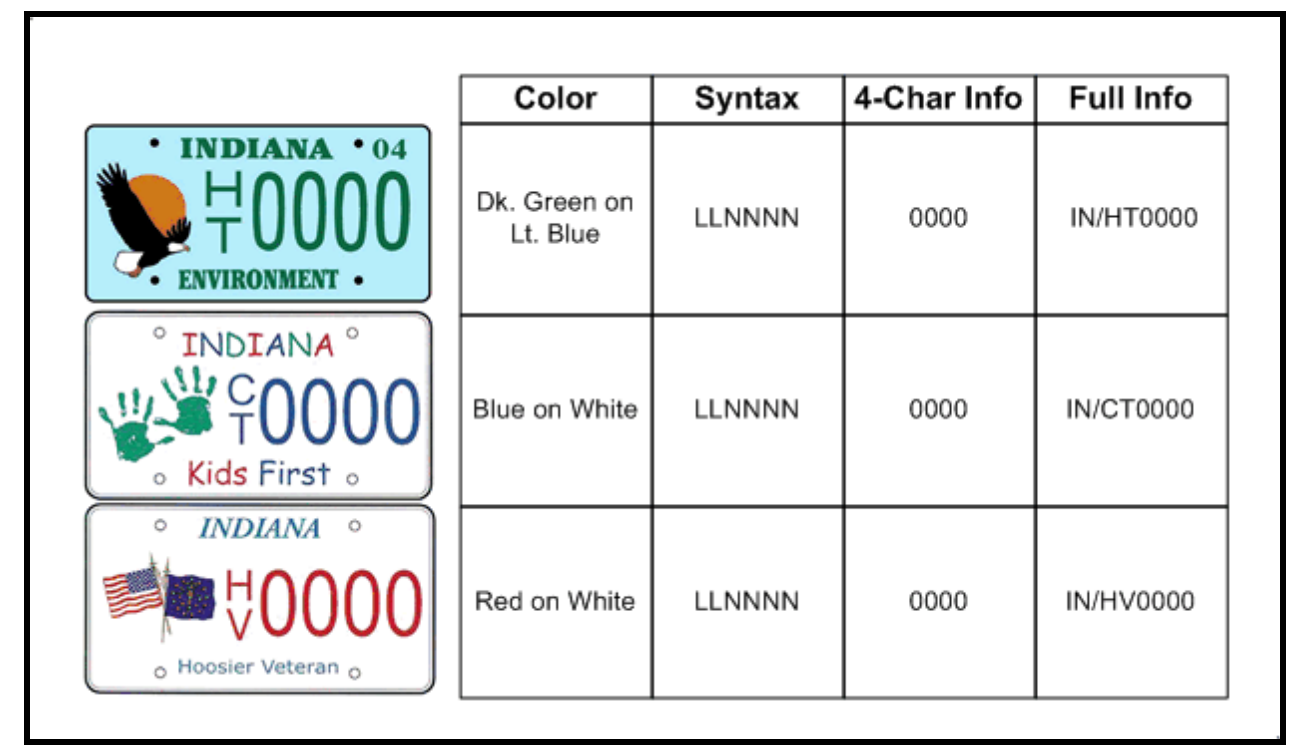

Figure 33: Indiana Passenger Vehicle Special Recognition License Plates Source of LP Images: Indiana Bureau of Motor Vehicles, 2005 
The general format of the special recognition plates issued by the Indiana BMV is the same for all organizations. All contain a unique logo on the left, followed by two stacked letters that are also unique to the organization (the stacked letters "HT" will not appear on any other special recognition plate besides the environment plate), followed by one, two, three, or four digits.

Many other states also issue special recognition plates. They may or may not have a similar format as Indiana. Therefore, if a license plate follow-up survey technique is being conducted using the clipboard, audio, or laptop methods, it may be difficult to obtain the state that issued that license plate, because it is less likely that the observer will recognize the state issuing the license plate.

\subsubsection{Indiana Commercial \& Government Vehicle License Plates}

In addition to the passenger vehicle license plates discussed above, other license plate variations are issued to commercial and government vehicles. Figure 34 illustrates the different variations of these.

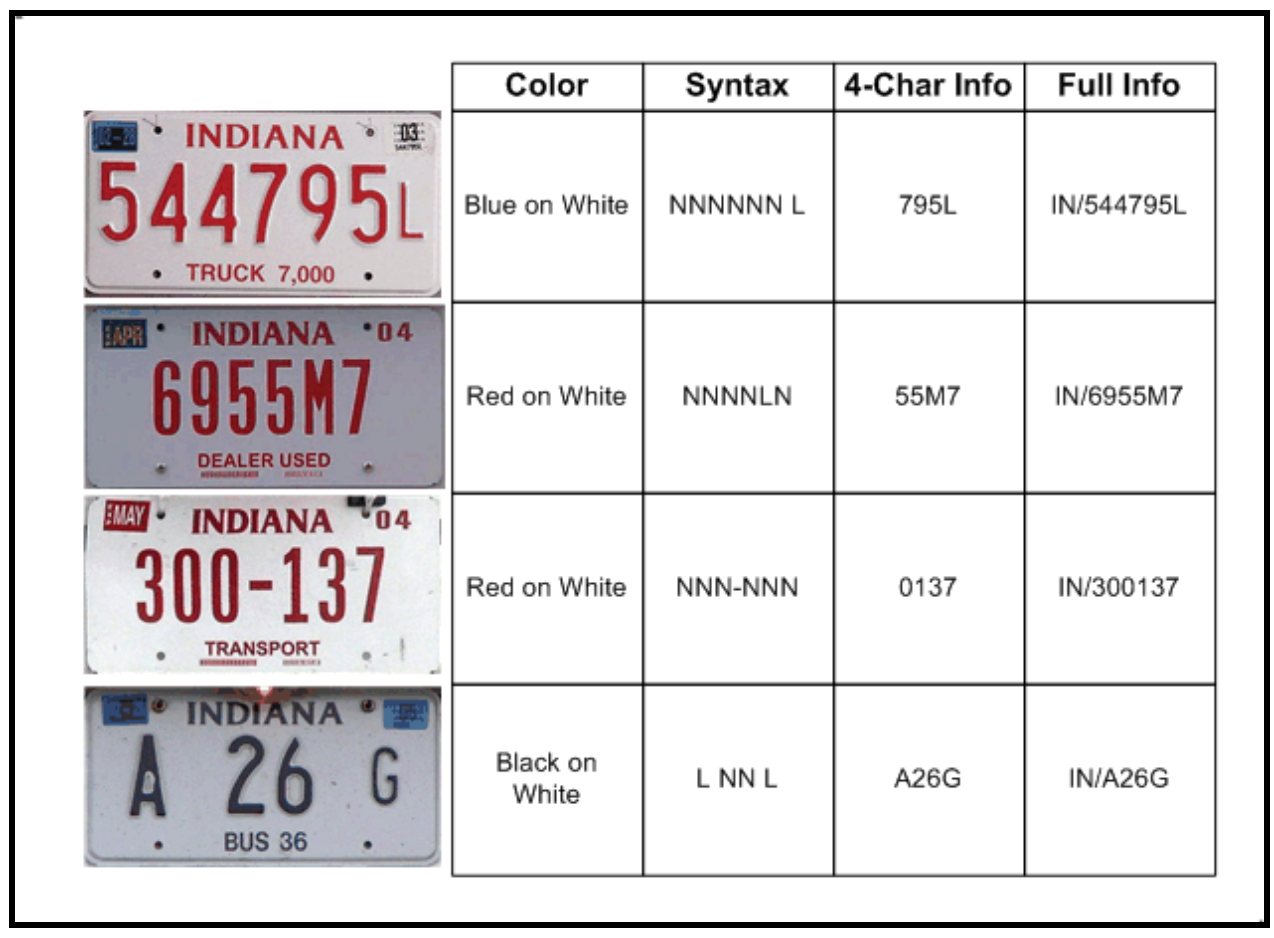

Figure 34: Indiana Commercial \& Government Vehicle License Plates Source of LP Images: Welby, 2005; Not all syntaxes shown for each vehicle type 


\subsubsection{Out-of-State License Plates}

Like Indiana, other states generally issue a standard passenger vehicle plate, special recognition plates, commercial vehicle plates, and government-owned license plates. While the height of the characters on the license plate is consistent, the font of the characters and the size and location of the state's name vary. In addition, the state name is sometimes covered by license plate frames. While this is not a problem for the license plate matching technique, it may become a problem for the license plate followup survey technique. In some cases, such as passenger vehicle plates, the state can be determined solely by the unique style of the plate. However, for commercial, government, and other generic plates, this may not be so. Figures 35 and 36 illustrate the formats of the standard passenger vehicle and commercial vehicle license plates for states surrounding Indiana.

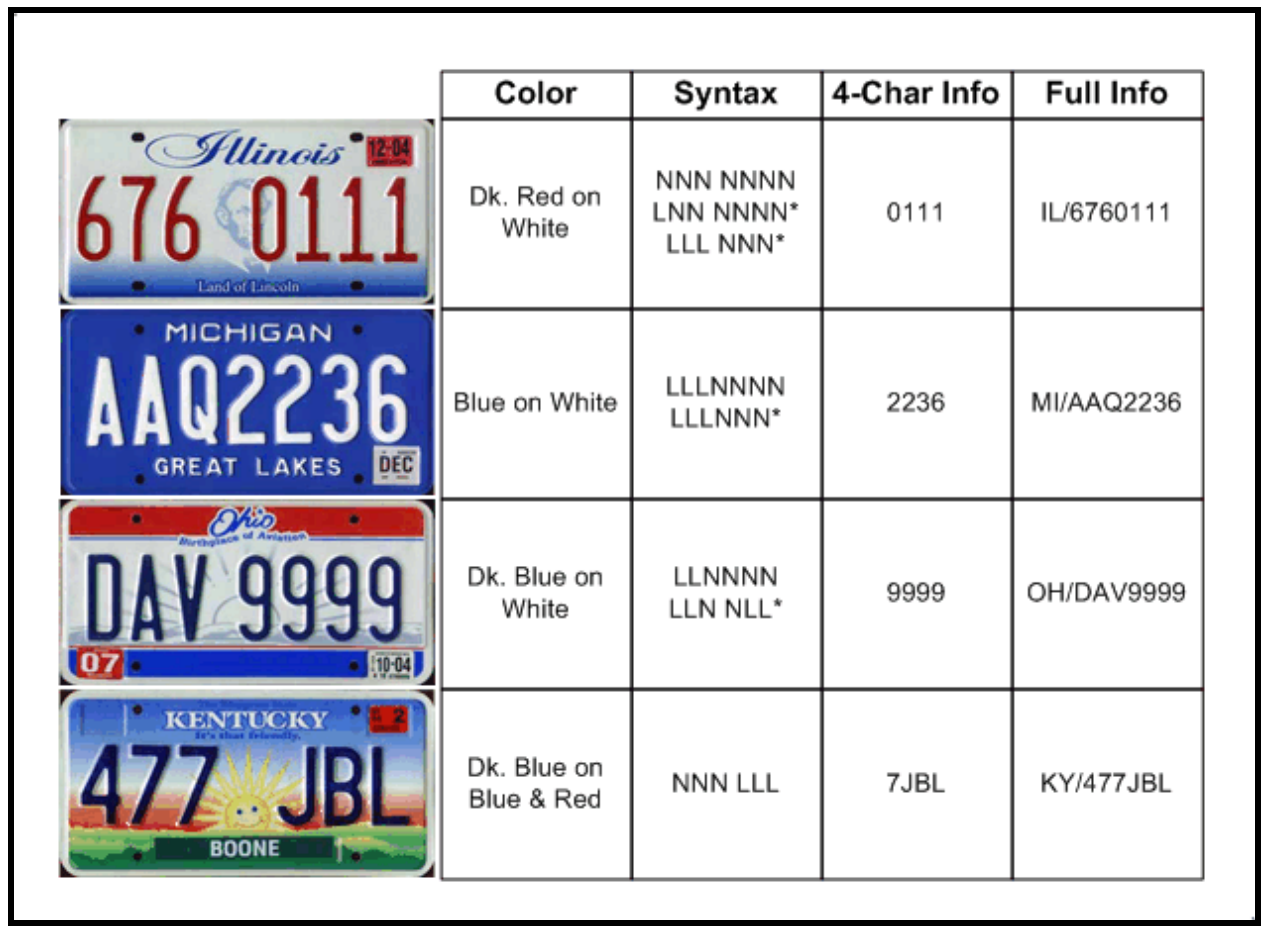

Figure 35: Out-of-State Standard Passenger Vehicle License Plates Source of LP Images: Nicholson, 2005 


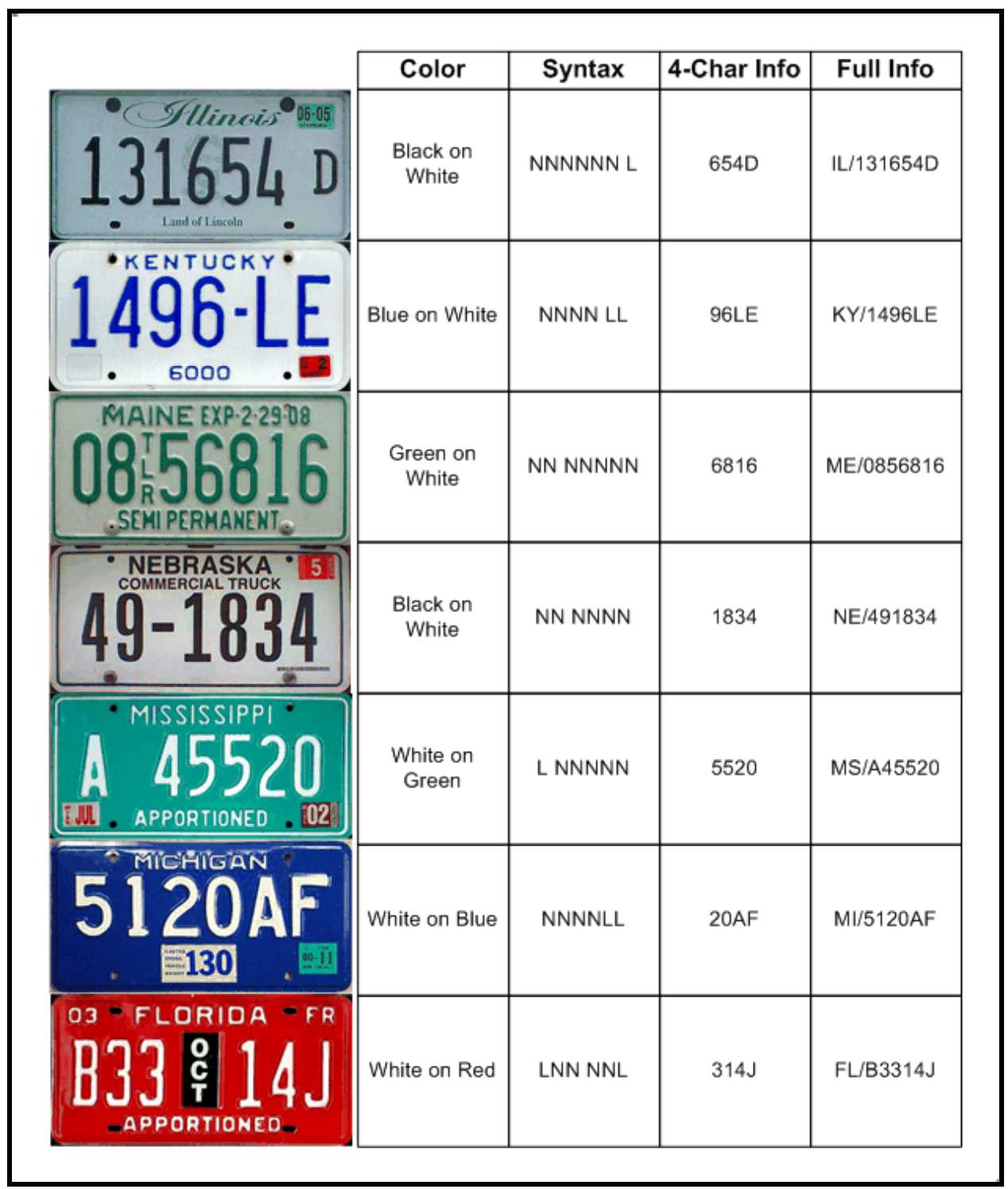

Figure 36: Out-of-State Commercial Vehicle License Plates Source of LP Images: Welby, 2005

\subsection{License Plate Identification in the Field}

Identifying the license plate string is the first step in the process of recording it. It is common to all of the methods. However, for the clipboard, audio, and laptop methods, this step is completed on the roadside during the study. The process of identifying the license plate string can be broken down into further sub-processes. To illustrate these sub-processes more clearly, imagine standing on the side of the road as vehicles pass by. In order to identify the license plate string of a passing vehicle, the following needs 
to be done: 1) locate the license plate on the back of the vehicle, 2) locate the first character or fourth-to-last character on the license plate, and 3) identify (and memorize) the license plate string (and state, if applicable). The reason the string has to be memorized is that, depending on the speed of the vehicle and the recording method being used, it is unlikely the license plate string will be visible at a second glance (because the vehicle will have traveled beyond the limit of legibility).

The value of this identification time is unknown, but important for a number of reasons (as will be discussed later in this chapter). Therefore, it is important that it be measured.

The amount of time required to identify a license plate string is dependent upon a number of things: the capabilities and condition of the person identifying the string (such as age, familiarity, and fatigue), the location of the license plate on the vehicle, the type of vehicle (passenger car, motorcycle, truck, trailer), the characteristics of the license plate such as the contrast between background and digit colors, and characteristics of the string such as font, color, syntax. In addition, the mix of vehicles on the roadway will have some effect on the identification time, compared to an ideal (but unlikely) situation in which all vehicles on the roadway have the same license plate style and syntax.

The string identification time is important for several reasons. First, the length of time that a license plate is legible to an observer on the roadside should be greater than the string identification time. In other words, vehicles should be traveling slowly enough or the observer should have visual acuity that is strong enough to enable him or her to see the license plate string legibly for at least as long as it will take him or her to identify it. Figure 37 illustrates this distance.

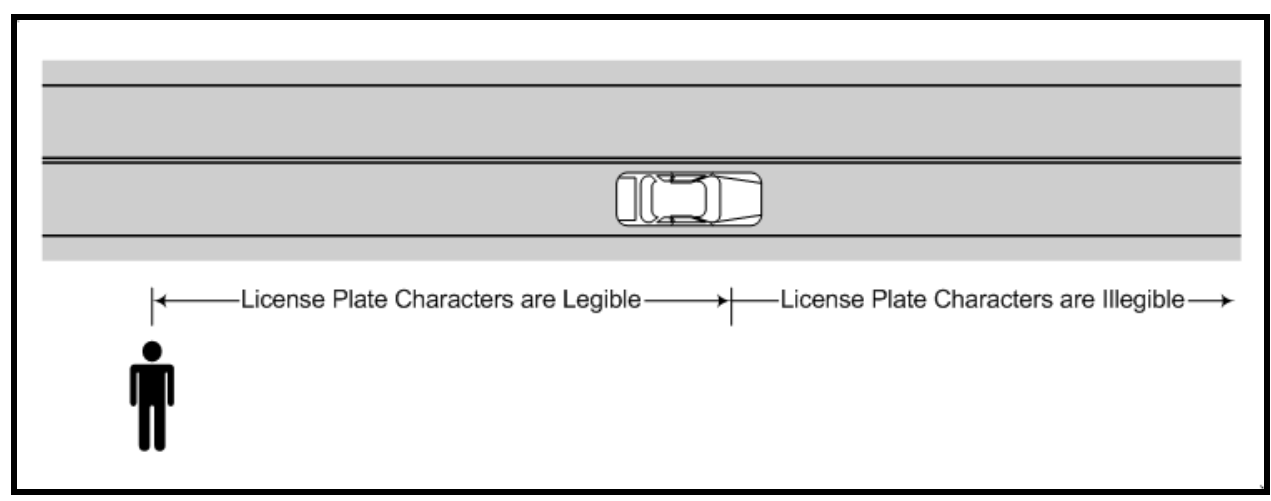

Figure 37: License Plate Legibility Distance 
Secondly, in order to speed up the manual transcription process of license plate strings recorded via video, it is desirable (although not required or even possible in some cases) to have the license plate string be visible and legible in the field-of-view for at least as long as the string identification time. By doing so, the video tapes can be transcribed at normal speed rather than in slow motion or pausing on each vehicle.

Because the string identification time is unknown, it had to be measured. The string identification time can be defined as the time that elapses from when an observer begins to search for a license plate on the back of vehicle until the observer has identified and is ready to record the string to some medium.

As an observer on the roadside trying to identify license plate strings, the search for the plate does not begin until the vehicle is some distance downstream from where the observer is standing. This is because the license plate is easier to read when it is moving along our line-of-sight. As the vehicle is just passing the observer, the license plate is moving almost perpendicular to the observer's line-of-sight, which makes it very difficult to read, especially at higher vehicle speeds. In addition, the license plate is not directly facing the observer at this point (because the observer is on the side of the road). Therefore, it is assumed that, if the observer is standing six feet from the lane edge, he or she begins to search for the license plate when the vehicle is approximately 20-25 feet downstream from where the observer is standing.

The string identification time ends when the observer has identified and memorized the license plate string. The observer has to memorize the string before recording because license plate string may not be legible by the time the observer looks a second time, usually because the license plate will be too far away and beyond the limit of the observer's visual acuity.

It was determined that the most accurate way to measure this string identification time was to have the researcher use a self-operated stopwatch. The reason the times were self-measured is because the researcher is the most accurate judge at which the string identification time begins and ends. As a vehicle passed, the researcher began the stopwatch as he or she began to look at the back of the vehicle. The researcher stops the time just as the last piece of information on the license plate is identified. The time was recorded, and the procedure repeated 50 times. These measurements were 
taken on roadways with three different speeds (15 mph, $35 \mathrm{mph}$, and $55 \mathrm{mph}$ ) from a location six feet from the adjacent lane edge with a line of sight of approximately 30 degrees from a line parallel to the road. The results of this data collection are presented in Sections 6.3.2 and 6.3.3.

Even if all vehicles were traveling at a speed slow enough and the observer has a visual acuity that is strong enough, some vehicles will be unidentifiable because of missing, damaged, dirty, covered, or blocked license plates. These vehicles are not considered in the distribution of identification times. For any given roadway, a small percentage of vehicles will be unidentifiable which, if unknown, introduces error when expanding the data. In this case, if a vehicle is unidentifiable at one observation station it is also unidentifiable at the next observation station. This is unlike another type of error, recording error, which will be discussed in Section 6.6. With recording error, an incorrectly recorded vehicle at one observation station is independent of it being incorrectly recorded at another observation station.

The percentage of vehicles that are unidentifiable due to missing, damaged, dirty, covered, or blocked license plates may vary from roadway to roadway; however, it is not expected to be large. The estimate made as a result of this study from several roadway observations is approximately $5 \%$ of all vehicles.

\subsubsection{Time-Length of License Plate Legibility}

The length of time a license plate on a passing vehicle is legible to an observer standing on the side of the road depends upon: 1) the speed of the vehicle, 2) the visual acuity of the observer, and 3 ) the size and style of the digits on the license plate. Visual acuity is stated as $20 / X$, where 20 is the distance (in feet) at which you can see an object, and $X$ is the distance a person with "normal" vision can see that same object. Therefore, a person with 20/20 vision has normal vision. A denominator less than 20 means better-than-average visual acuity, and a denominator greater than 20 means worse-than-average visual acuity. At 20/200, a person is considered legally blind.

$20 / 20$ visual acuity is defined as the distance at which a person can see an object that spans 5 minutes of arc (5') or $1 / 12$ of a degree. Figure 38 illustrates this. 


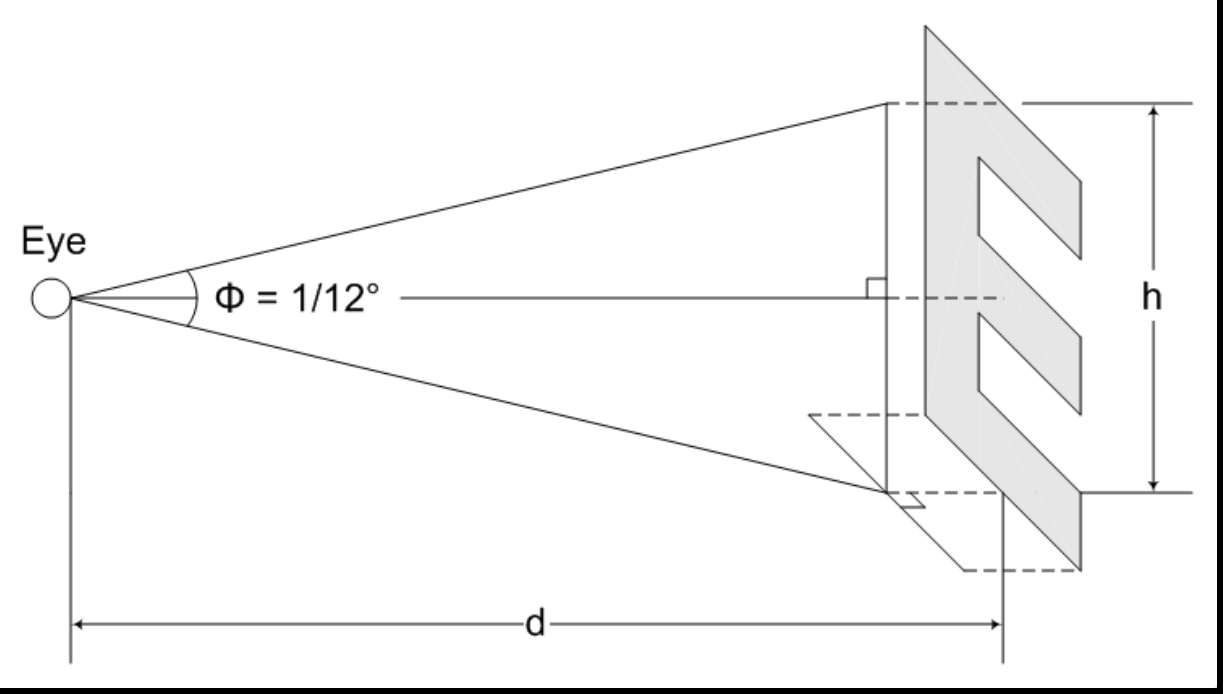

Figure 38: Definition of 20/20 Visual Acuity

Therefore, by solving for $d$ in Figure 38, a person with 20/20 visual acuity will theoretically be able to identify the characters on a license plate (approximately 2.75 " in height) at a distance of 158 feet. A person with 20/10 vision will be able to see the characters at twice that distance (316 feet), and a person with 20/40 vision will be able to see the characters at half that distance (79 feet).

Depending upon the speed of the vehicle and the visual acuity of the observer, a license plate will remain legible for a short period of time after it passes the observer. Table 8 summarizes these times.

Table 8: Theoretical Time-Length of License Plate Legibility (2.75" Characters)

\begin{tabular}{|c|c|c|c|c|c|c|c|c|c|}
\hline Visual & Distance & \multicolumn{8}{c|}{ Vehicle Speed (mph) } \\
\cline { 3 - 11 } Acuity & Leg. (ft) & 5 & 15 & 25 & 35 & 45 & 55 & 65 & 75 \\
\hline \hline $20 / 10$ & 316 & 43.1 & 14.4 & 8.6 & 6.2 & 4.8 & 3.9 & 3.3 & 2.9 \\
\hline $20 / 15$ & 211 & 28.7 & 9.6 & 5.7 & 4.1 & 3.2 & 2.6 & 2.2 & 1.9 \\
\hline $20 / 20$ & 158 & 21.5 & 7.2 & 4.3 & 3.1 & 2.4 & 2.0 & 1.7 & 1.4 \\
\hline $20 / 30$ & 105 & 14.4 & 4.8 & 2.9 & 2.1 & 1.6 & 1.3 & 1.1 & 1.0 \\
\hline $20 / 40$ & 79 & 10.8 & 3.6 & 2.2 & 1.5 & 1.2 & 1.0 & 0.8 & 0.7 \\
\hline
\end{tabular}

It can be seen, then, that a license plate string is legible to an observer with 20/10 vision on a vehicle traveling at $5 \mathrm{mph}$ for $43.1 \mathrm{~s}$ after the vehicle passes.

However, that same license plate string is only legible to an observer with 20/40 vision for $10.8 \mathrm{~s}$. Likewise, a license plate string is legible to an observer with $20 / 20$ vision on a 
vehicle traveling at $5 \mathrm{mph}$ for $21.5 \mathrm{~s}$. However, a license plate is only legible to the same observer (with 20/20 vision) for $1.4 \mathrm{~s}$ if the vehicle is traveling $75 \mathrm{mph}$. This table illustrates that time of legibility decreases as vehicle speed increases and visual acuity decreases.

Many of the Indiana license plates also use characters that are half the size as the normal characters. In many cases, these characters also have to be identified and recorded in accordance with the rules described in Section 6.1. Therefore, Table 9 illustrates the legibility distance for these half-sized characters and the length of time they are legible from the roadside.

Table 9: Theoretical Time-Length of License Plate Legibility (1.25" Characters)

\begin{tabular}{|c|c|c|c|c|c|c|c|c|c|}
\hline Visual & Distance & \multicolumn{8}{c|}{ Vehicle Speed (mph) } \\
\cline { 3 - 12 } Acuity & Leg. (ft) & 5 & 15 & 25 & 35 & 45 & 55 & 65 & 75 \\
\hline \hline $20 / 10$ & 143 & 19.5 & 6.5 & 3.9 & 2.8 & 2.2 & 1.8 & 1.5 & 1.3 \\
\hline $20 / 15$ & 95 & 13.0 & 4.3 & 2.6 & 1.9 & 1.4 & 1.2 & 1.0 & 0.9 \\
\hline $20 / 20$ & 72 & 9.8 & 3.3 & 2.0 & 1.4 & 1.1 & 0.9 & 0.8 & 0.7 \\
\hline $20 / 30$ & 48 & 6.5 & 2.2 & 1.3 & 0.9 & 0.7 & 0.6 & 0.5 & 0.4 \\
\hline $20 / 40$ & 36 & 4.9 & 1.6 & 1.0 & 0.7 & 0.5 & 0.4 & 0.4 & 0.3 \\
\hline
\end{tabular}

After calculating the table above and performing some field data collection, it was realized that the characters on license plates that were the most difficult to read (the standard license plate shown in Figure 32) were, in fact, not legible at these distances. Therefore, knowing that the visual acuity of the researcher is $20 / 15$, the legibility distance of standard Indiana license plates were measured on stationary vehicles. The legibility distance of the small characters on the standard Indiana passenger vehicle license plate was 72 feet (instead of 95 feet calculated in the table above). Therefore, the legibility distances for other visual acuities were determined and the time lengths of license plate legibility were recalculated using these values.

Table 10: Observed Time-Length of License Plate Legibility (1.25" Characters)

\begin{tabular}{|c|c|c|c|c|c|c|c|c|c|}
\hline Visual & Distance & \multicolumn{8}{c|}{ Vehicle Speed (mph) } \\
\cline { 3 - 11 } Acuity & Leg. (ft) & 5 & 15 & 25 & 35 & 45 & 55 & 65 & 75 \\
\hline \hline $20 / 10$ & 108 & 14.7 & 4.9 & 2.9 & 2.1 & 1.6 & 1.3 & 1.1 & 1.0 \\
\hline $20 / 15$ & 72 & 9.8 & 3.3 & 2.0 & 1.4 & 1.1 & 0.9 & 0.8 & 0.7 \\
\hline $20 / 20$ & 54 & 7.4 & 2.5 & 1.5 & 1.1 & 0.8 & 0.7 & 0.6 & 0.5 \\
\hline $20 / 30$ & 36 & 4.9 & 1.6 & 1.0 & 0.7 & 0.5 & 0.4 & 0.4 & 0.3 \\
\hline $20 / 40$ & 27 & 3.7 & 1.2 & 0.7 & 0.5 & 0.4 & 0.3 & 0.3 & 0.2 \\
\hline
\end{tabular}


There are several reasons the characters are not legible at the theoretical distances calculated in Table 9. First, some characters are easier to see than others. Also, the characters do not appear on a single solid background color. More importantly, the characters are thinner (skinnier) than characters used to measure visual acuity. Finally, the visual acuity of the observer may not be exactly 20/15.

\subsubsection{License Plate Identification Time}

After the license plate identification times were measured in the field, the data was analyzed to determine if the speed of the vehicle had any effect on the license plate identification time. Table 9 illustrates the means and standard deviations of the identification time for each parameter.

Table 11: Identification Time (s) by Vehicle Speed

\begin{tabular}{|c|c|c|c|c|}
\hline \multirow{2}{*}{ Speed } & \multicolumn{2}{|c|}{ 4-Character } & \multicolumn{2}{c|}{ Full String } \\
\cline { 2 - 5 } & Mean & St. Dev. & Mean & St. Dev. \\
\hline \hline $15 \mathrm{mph}$ & 0.77 & 0.16 & 1.36 & 0.28 \\
\hline $35 \mathrm{mph}$ & 0.75 & 0.13 & 1.02 & 0.21 \\
\hline $55 \mathrm{mph}$ & 0.86 & 0.16 & 1.08 & 0.23 \\
\hline
\end{tabular}

It can be seen from the table that, in general, the mean identification time increases as vehicle speed increases. The primary exception to this was the mean identification value of a full string at $15 \mathrm{mph}$ (1.36 s), although the 4-character identification time for the same parameters also seemed slightly high at $0.77 \mathrm{~s}$. These higher values may be explained by the fact that these values were the first to be measured in the field, and the researcher became slightly better at identifying vehicles over time. Another possibility is that, at lower vehicle speeds, the time length of legibility is longer, so identifying vehicles quickly is not as critical as it is at higher vehicle speeds because the observer has enough time to take a second look at the license plate.

\subsubsection{Probability of String Identification}

Because the ID times are approximately normally distributed, the probability that a passing license plate will be recorded based on vehicle speed and visual acuity can be determined by plotting the time lengths of legibility calculated in Table 10 on the ID time 
distributions using the means and standard deviations in Table 11 and obtaining the percentiles. These percentiles are the probability that a license plate will be recorded at a given speed.

However, because it is known that the mean identification time varies with vehicle speed, different distributions must be drawn for different vehicle speeds. The mean identification time for each vehicle speed was determined by conducting a linear regression on the mean identification times collected in the field. (The full string mean value at $15 \mathrm{mph}$ was not included in the regression because it was considered unusually high.)

Figures 39 through 40 below illustrate the probability of identifying a standard issue Indiana passenger vehicle license plate (with 1.25" characters) on a vehicle at any speed for five different visual acuities ranging from $20 / 10$ to $20 / 40$. Figure 39 is for 4 character string identification and Figure 40 is for full string identification.

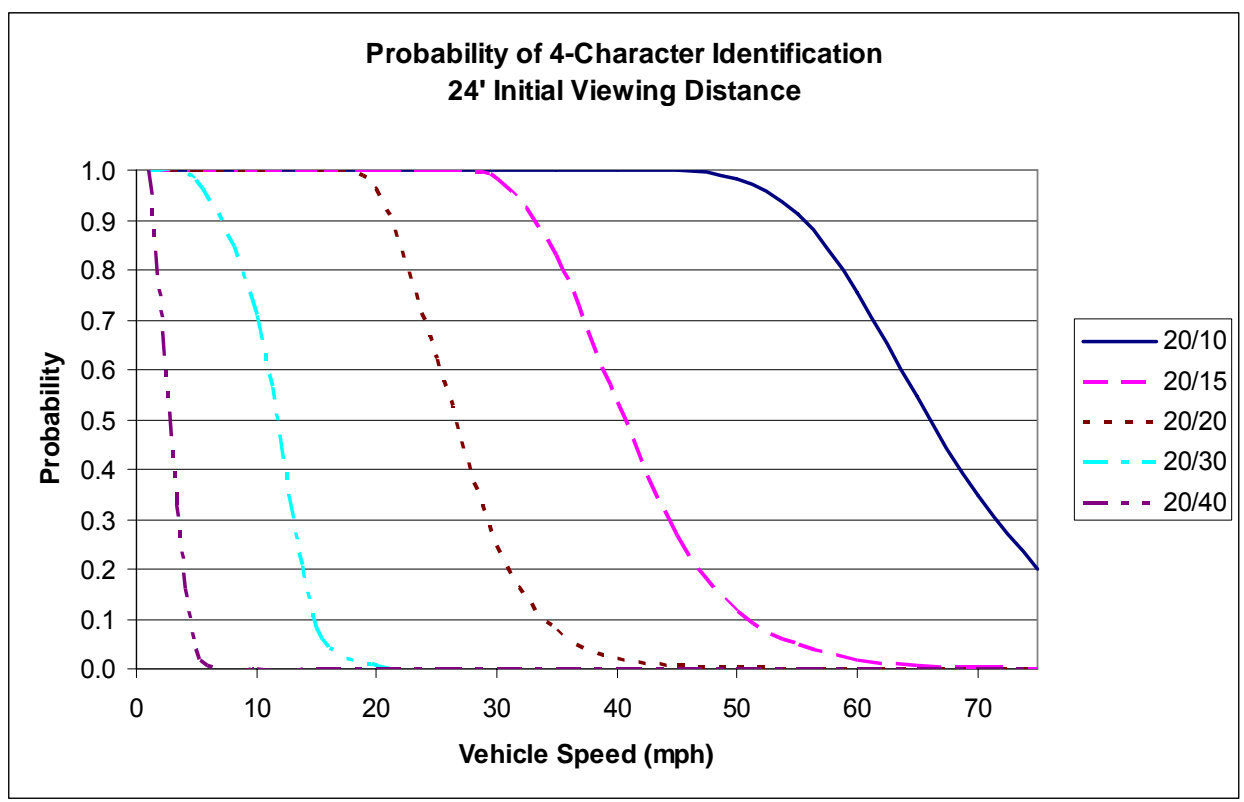

Figure 39: Probability of 4-Character Identification 


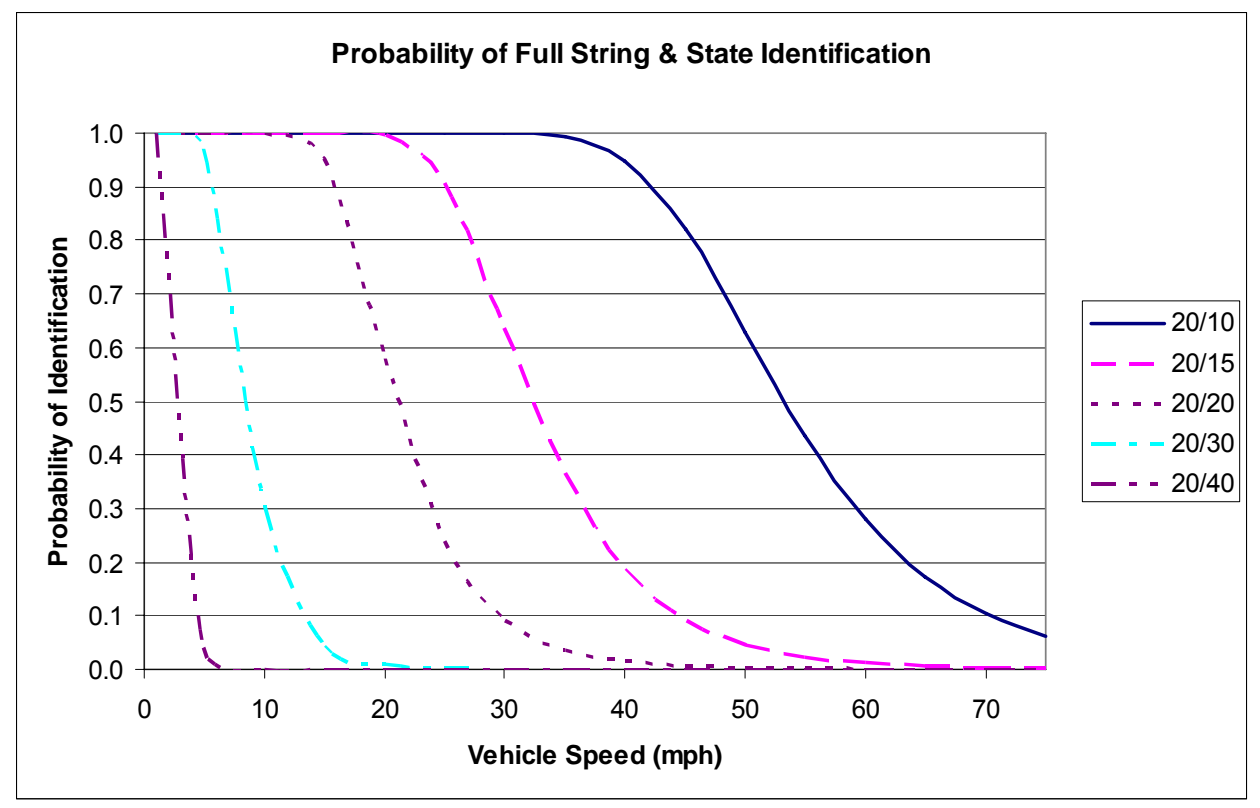

Figure 40: Probability of Full String Identification

These figures were constructed based on the analysis of mean identification times for one researcher. Naturally, variations in identification time also exist from person to person. Initially, it was determined that data on identification times from other persons be obtained. However, because of the complexity involved in measuring identification times using a self-operated stopwatch, the difficulty in recruiting a variety of persons for the task, and the length of time required to record the data, data was only obtained from one person.

\subsubsection{Maximum Vehicle Speed for Manual License Plate Data Collection}

In the previous section, the identification time, which is the time required to locate and memorize a license plate string, was determined. This identification time should not exceed the time-length of legibility for any given roadway speed and visual acuity. If so, the license plate string on a vehicle traveling away from the observer will become illegible before the observer has enough time to identify the string. Therefore, we can calculate the maximum vehicle speed at which an observer with a specific visual acuity should attempt to record license plate strings using manual methods.

Table 12 recommends the vehicle speed above which manual methods (clipboard, audio, and laptop) should not be used. Instead, the video method should be 
used. These speeds in Table 12 are the points at which the probability curve for each visual acuity begins to drop from 1.0 .

Table 12: Vehicle Speed (mph) above which Video Method is Recommended

\begin{tabular}{|c|c|c|c|c|}
\hline \multirow{2}{*}{ Visual Acuity } & \multicolumn{2}{|c|}{$1.25 "$ Characters } & \multicolumn{2}{c|}{$2.75 "$ Characters } \\
\cline { 2 - 5 } & 4-Character & Full String & 4-Character & Full String \\
\hline \hline $20 / 10$ & 45 & 35 & 100 & 70 \\
\hline $20 / 15$ & 30 & 20 & 65 & 45 \\
\hline $20 / 20$ & 20 & 10 & 45 & 30 \\
\hline $20 / 30$ & 5 & n/a & 25 & 20 \\
\hline $20 / 40$ & n/a & n/a & 15 & 10 \\
\hline
\end{tabular}

It can be seen in Table 12 that, even with 20/10 visual acuity, recording 4character license plates with a manual method is not recommended for vehicle speeds greater than $45 \mathrm{mph}$. For an observer with $20 / 20$ visual acuity, the maximum speed is $20 \mathrm{mph}$. For full string recording, manual methods may be used for vehicle speeds less than $10 \mathrm{mph}$ and visual acuity of $20 / 20$, or less than $30 \mathrm{mph}$ for visual acuity of 20/10. Again, this is only recommended for recording the near lane.

For vehicle speeds that exceed the maximum speed for identification with a manual method, video recording is likely the best method. Video recording was discussed in greater detail in Chapter 5.

If conducting an OD study on a subset of vehicles, such as trucks, the video method is recommended. This is because trucks may have multiple license plates mounted in different locations, and are often dirty and harder to see than passenger vehicle license plates. These factors generally make the identification time of a license plate on a truck higher than that of a passenger vehicle.

If video recording is not possible, other alternatives exist. For example, two personnel could be used at each station - one for observing vehicles with binoculars, and the second for recording the license plate strings. By doing this, the length of time a license plate is legible increases significantly, but twice as many people are needed in the field. This was not evaluated as part of this project. In addition, it may be possible to reduce the vehicle speed on the road through reduced speed limits (using work zone traffic control methods), but this would require permission and assistance from the DOT. 
In addition, police enforcement may be needed to ensure that motorists actually reduce speed enough to make the manual method successful.

\subsection{License Plate Recording}

From the above paragraphs, the maximum vehicle speed at which license plates can be identified was found for any visual acuity. Just as the identification time was determined in a preceding section, the recording time is also desired, which is the amount of time the observer takes to record the plate just identified into some recording medium. The recording time will differ for each of the recording methods (clipboard, audio, and laptop). Because the average recording time for one method is likely to be different than that of the other methods, each of the methods is likely to be limited by a minimum vehicle headway (and, conversely, maximum vehicular flow rate) at which a second vehicle will pass the observation point before the license plate of the previous vehicle is recorded, thereby missing the second vehicle. Furthermore, the recording time for a 4-character string is likely less than that of recording a full license plate string, so depending on the technique used (matching or follow-up survey), one method may not be suitable for both techniques. The objective of determining these recording times is to identify the method that enables the highest capture rate to be obtained. The capture rate is the number of license plates recorded divided by the total number of vehicles passing the observation station.

\subsubsection{License Plate Recording Time}

In order to determine these recording times, a procedure was used that was similar to that for measuring string identification times discussed earlier. In this case, the identification and recording times were measured together.

This time was measured by a researcher using a self-operated stopwatch. The timer began when the license plate entered the researcher's field-of-view and remained running until the last digit of the license plate string was recorded. This procedure was repeated 50 times, recording 4-character and full license plate strings by each method.

Table 13 illustrates the recording time mean and standard deviation for each method. 
Table 13: Recording Time (s) by Method

\begin{tabular}{|l|c|c|c|c|}
\hline \multirow{2}{*}{ Method } & \multicolumn{2}{|c|}{ 4-Character } & \multicolumn{2}{c|}{ Full String } \\
\cline { 2 - 5 } & Mean & St. Dev. & Mean & St. Dev. \\
\hline \hline Clipboard & 2.99 & 0.33 & 4.95 & 0.81 \\
\hline Audio & 1.76 & 0.25 & 2.84 & 0.50 \\
\hline Laptop & 2.51 & 0.49 & 5.38 & 1.05 \\
\hline
\end{tabular}

The average time to identify and record a 4-character license plate to a clipboard was $2.99 \mathrm{~s}$. Therefore, if the license plate of vehicle 1 is being identified and recorded, and vehicle 2 is following vehicle 1 with headway of less than the recording time, the license plate of vehicle 2 will not be recorded because the observer has not completed recording of vehicle 1 .

\subsubsection{Vehicle Interarrival Times (Headways)}

The probability that a vehicle will have a headway greater than a given value (the recording time) can be calculated by the equation $P(T \geq t)=e^{-\lambda t}$, where $\lambda$ is the flow rate in vehicles per second and $t$ is the headway in seconds. This equation is derived from the Poisson model of traffic flow and has a negative exponential distribution (Fricker \& Whitford, 2004).

For example, if the flow rate of a particular road is $400 \mathrm{veh} / \mathrm{h}(0.111 \mathrm{veh} / \mathrm{s})$ and the time to record a license plate is $2.99 \mathrm{~s}$, the probability that the headway between vehicles is greater than $2.99 \mathrm{~s}$ is $71.8 \%$.

\subsubsection{Maximum Flow Rate for Manual Methods}

The objective of this task is to specify a flow rate at which the video method should be used instead of manual methods (clipboard, audio, and laptop), because at higher flow rates, a higher percentage of vehicles will be missed. To do this, the joint probability density function was approximated. This was done by randomly selecting a recording time (assumed to be normally distributed and based on the mean and standard deviations in Table 13) and a vehicle headway (based on the negative exponential distribution in Section 6.4.2). These two values were compared, and the process was repeated 10,000 times for various flow rates. The percentage of the time that the recording time was less than the headway was determined. Figure 41 illustrates the percentage of vehicles that could be recorded, based on flow rate for each method. 


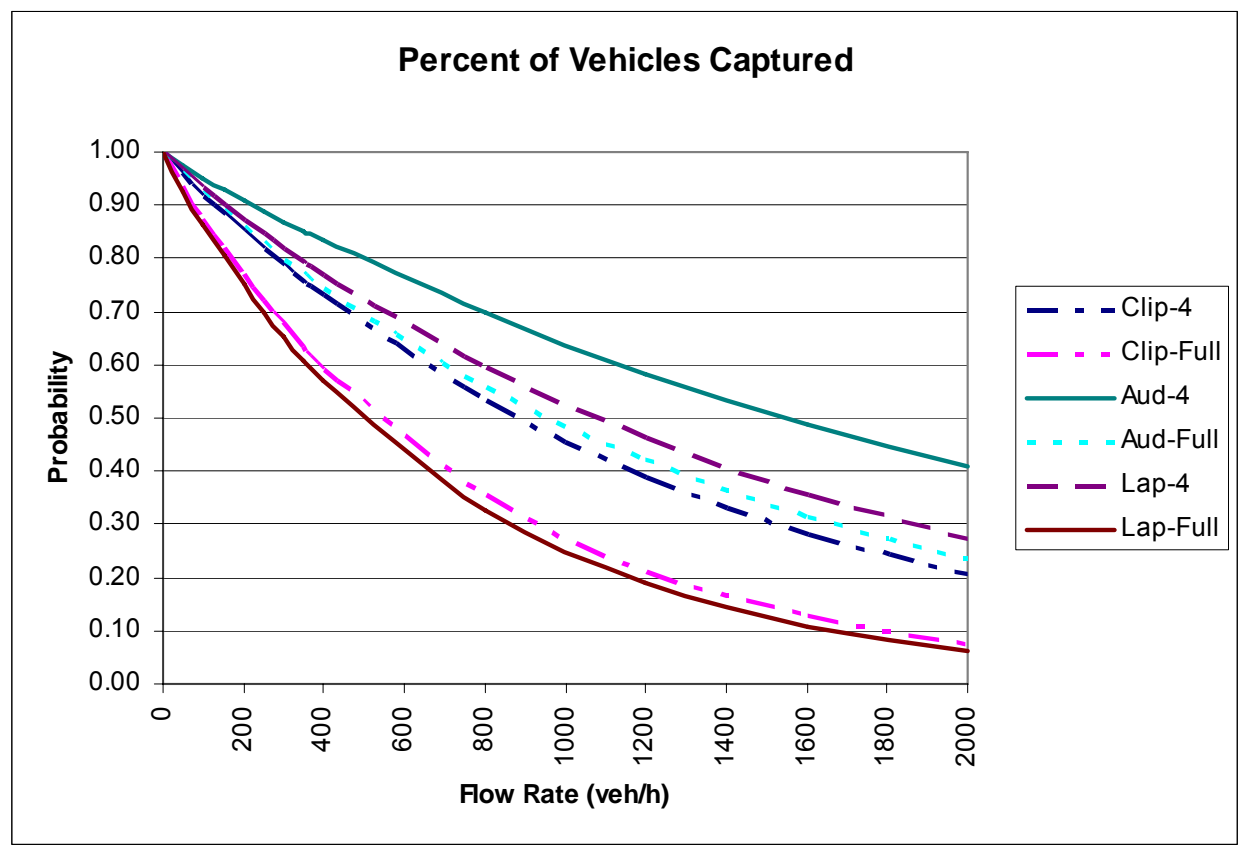

Figure 41: Capture Rates by Vehicle Flow for each Method

Using Figure 41, the percent of vehicles captured can be determined for any flow rate. If the capture rate is sufficient for the purpose of the study, that method can be used. If not, however, the video method is recommended. Table 14 summarizes the maximum flow rates for the $95 \%, 85 \%$, and $75 \%$ capture rates (rounded to the nearest 25 vehicles).

Table 14: Maximum Flow Rate (vphpl) using Manual Recording Methods

\begin{tabular}{|l|c|c|c|c|c|c|}
\hline \multirow{2}{*}{ Method } & \multicolumn{2}{|c|}{$95 \%$ Capture } & \multicolumn{2}{c|}{$85 \%$ Capture } & \multicolumn{2}{c|}{$75 \%$ Capture } \\
\cline { 2 - 7 } & $4-$ Char & Full & 4 -Char & Full & $4-$ Char & Full \\
\hline \hline Clipboard & 50 & 25 & 200 & 125 & 350 & 225 \\
\hline Audio & 100 & 50 & 350 & 225 & 650 & 375 \\
\hline Laptop & 75 & 25 & 250 & 100 & 450 & 200 \\
\hline
\end{tabular}

Table 14 illustrates that, in order to record 4 characters on $95 \%$ of all identifiable license plates using the audio method, the flow rate cannot exceed $100 \mathrm{vphpl}$. If the acceptable capture rate is reduced, the maximum flow rates increase significantly. For flow rates that exceed those shown in the table, the video method is recommended for recording license plates. 
This table only specifies flow rates for uninterrupted flow. If license plate data is being collected from a roadway with interrupted flow (e.g., downstream from a traffic signal), vehicles are more likely to be spaced closely together. This depends on the

amount of queueing at the signal. If there is a lot of queueing and vehicles are traveling past the observation station in platoons, the video method is recommended for data collection. In addition, this table does not account for observer fatigue.

Chapter 7 evaluates how the capture rates affect the overall accuracy of the estimated OD pairs.

\subsection{Video Method}

Based on the preceding sections, manual recording of license plate strings with the clipboard, audio, and laptop methods may not suffice in situations with high vehicle speeds and/or high flow rates. In these cases, video can be used to record the license plates. The video can then be played back at a controlled speed in the lab to obtain more license plate strings than would be obtained using any other method for that roadway. However, the legibility of license plate strings is dependent on the quality of the video. There are a number of issues to consider when using a camcorder to obtain the best possible video quality, including the distance from the roadside, the angle, and zoom. In addition, lighting conditions, vehicle speeds, and flow rates should also be considered. These issues were discussed in detail in Chapter 5. The goal of the following section is to evaluate the process of extracting the appropriate information from the clipboard, audiotape, laptop, videotape after field collection has been completed.

\subsection{Errors in License Plate Data}

Obviously, the objective in recording license plates is to record as many as possible accurately. However, it is unlikely that an observer will make no mistakes in recording the data in the field. While the capture rate was discussed in the previous section, the error in recording these license plates is discussed in this section.

\subsubsection{Field and Transcription Errors}

Several experiments were conducted to determine the field and transcription errors for each of the data collection methods. Field errors are errors that are encountered in the field as the license plate strings are identified and recorded from the 
vehicle onto the clipboard, audio tape, laptop, or videotape. Transcription errors, on the other hand, are errors that are made after the data collection period while transferring the data in its raw form on the recording medium into some database.

For each of the clipboard, audio, and laptop methods, 100 4-character and full license plate strings were recorded. During each of these sessions, a camcorder was also set up and taped the same set of vehicles on the roadway. At the end of each session, the data recorded on the clipboard, audio tape, and laptop were transcribed into separate spreadsheets. Likewise, the video was reviewed, and all license plates strings were transcribed into the appropriate spreadsheets. This transcription process was repeated for each session. In the end, the spreadsheet for each session contained each license plate four times: two columns of the license plate records from the clipboard or audio tape, and two columns from the analysis of the corresponding video. In the case of the laptop, there are only three columns because the license plates are entered directly into the computer in the field and do not require transcription.

By transcribing all of the data twice, the field and transcription errors, as well as transcription time can be determined for each of the methods. These errors can occur when the license plate is misread. An example of this type of error is mistaking an " $O$ " for a "Q", "6" for an "8", and "M" for an "N". These errors can also occur when the characters are identified correctly, but then incorrectly recorded. Examples of this type of error include transposing letters ("68" instead of "86), speaking too quickly in the audio recorder, and incorrect key entry on the laptop. Table 15 summarizes the errors for each of the methods.

Table 15: Field \& Transcription Errors by Method

\begin{tabular}{|l|c|c|c|c|}
\hline \multirow{2}{*}{ Method } & \multicolumn{2}{|c|}{ Field } & \multicolumn{2}{c|}{ Transcription } \\
\cline { 2 - 5 } & 4-Char & Full & 4-Char & Full \\
\hline \hline Clipboard & $0.0 \%$ & $3.0 \%$ & $0.0 \%$ & $1.5 \%$ \\
\hline Audio & $3.0 \%$ & $4.0 \%$ & $1.5 \%$ & $0.5 \%$ \\
\hline Laptop & $2.9 \%$ & $3.7 \%$ & $\mathrm{n} / \mathrm{a}$ & $\mathrm{n} / \mathrm{a}$ \\
\hline Video & $\mathrm{n} / \mathrm{a}$ & $\mathrm{n} / \mathrm{a}$ & $0.3 \%$ & $0.7 \%$ \\
\hline
\end{tabular}

From the table above, the total amount of error for any particular method after field recording and transcribing the data is the sum of the two values shown. For example, if full strings of the license plates are field recorded using the audio method 
and subsequently transcribed, the total amount of error will be $4.5 \%$ (4.0\% from the field and $0.5 \%$ from the transcription). These errors, in effect, reduce the capture rates even further, and must be considered when expanding the sample data to the entire population. This is discussed in Chapter 7.

The 4-character recording error for all methods is generally less than the full string recording error. It seems logical that an observer is more likely to make a mistake when recording more characters. To illustrate, it is generally easier to remember the last four digits of a phone number than it is the entire phone number.

The clipboard method had the least amount of field error among the three methods. Generally, fewer errors are made with the clipboard than when keying entries into a laptop. However, unlike the clipboard and audio methods, the laptop does not have an extra transcription step, which adds another element of error to the first two methods.

The audio method had the most field error of the three methods. This could be because, unlike the clipboard or laptop methods, the audio method does not require the observer to take his or her eyes off the traffic, thus enabling him or her to continuously record license plates. In some cases, the license plates appear faster than the observer should attempt to record, which may cause the observer to make more of the mistakes described above, or make transcription difficult because the characters are recorded too quickly to understand during playback.

For the laptop method, during field recording, license plate entries were keyed into the laptop using the standard laptop keyboard with the numbers along the top. During the transcription process, license plate entries were keyed in using a keyboard with a number pad. This partially explains why the errors for field recording with a laptop are greater than the transcription errors for all other methods. Generally, it is easier to type numbers on a number pad rather than along the top of the keyboard, and transcription is done indoors in a more comfortable environment.

These field recording errors were measured under low speed conditions, and do not reflect errors that may be encountered if manual recording is conducted on a speed higher than those recommended by Table 12. In such cases, the error will likely be significantly higher, which reduces the overall accuracy when expanding the data.

Transcription errors for each of the methods are generally lower than field errors. These errors are generally caused by poor handwriting (for the clipboard method), poor 
audio such as background noise (for the audio method), and poor video quality (for the video method). Again, license plates recorded by a laptop in the field do not require transcription.

When these errors occur, there is the possibility (for the license plate matching technique) that some license plates that should be matched to another location will not be matched. Therefore, the number of matches need to be increased to reflect these errors. Likewise, for the follow-up survey technique, the license plate will not provide the correct vehicle owner address (or an address at all) by searching the BMV database. Therefore, the number of surveys sent out will be less than the number of license plates recorded in the field, which will reduce the overall accuracy of the survey results.

While the true error is not known in an actual OD study, the values summarized in Table 15 may be used as a guideline when expanding the data only if, for manual methods, the data was not collected from vehicle speeds that exceeded those recommended in Table 12.

\subsubsection{Transcription Time}

In addition to the variation in errors among the methods, the amount of time required to transcribe the license plates varies significantly from method to method. This does not influence the overall quality of the results, but certain methods will require more time (and more cost). Automatic transcription can be used to reduce this transcription time. However, the added expense of automatic transcription technology and the generally lower capture rate must also be considered. Again, automatic transcription technologies were not evaluated as part of this project.

The total transcription time for each of the methods was simultaneously measured from the same license plate data used to determine errors. Table 16 summarizes these transcription times. The coefficients that appear in Table 16 were determined by dividing the transcription time by the number of license plates transcribed. The table does not account for long-term effects, such as fatigue, but are recommended for estimating transcription times. 
Table 16: Transcription Time (sec) by Method

\begin{tabular}{|l|c|c|}
\hline Method & 4-Char & Full \\
\hline \hline Clipboard & $2.4 n$ & $4.2 n$ \\
\hline Audio & $L+0.3 n$ & $L+1.2 n$ \\
\hline Laptop & n/a & n/a \\
\hline Video & $L+3.5 n$ & $L+6.3 n$ \\
\hline
\end{tabular}

For the clipboard method, the transcription rate is simply the total time required to transcribe $n$ license plates divided by the number of license plates. Therefore, if 10,000 4-character license plates are recorded during an OD study, it will take approximately 6.7 hours $(2.4 * 10,000 / 3600)$ to manually transcribe them.

For the audio and video methods, the transcription time required consists of $L$, which is the total time length (in seconds) of audio or video tape recorded in the field plus the average time paused per license plate times the number of license plates recorded. For example, if 10,000 4-character license plates were recorded on 8 hours of videotape, the approximate manual transcription time is 17.7 hours $\left(\left[8^{*} 3600+3.5 * 10,000\right] / 3600\right)$.

Generally, the video method has the highest transcription time. However, it must be noted that video also has higher capture rates and lower errors than the other methods. 


\section{CHAPTER 7 - ACCURACY OF OD STUDY TECHNIQUES}

While the previous chapter discusses traffic conditions as they relate to a license plate survey, it is often unknown how accurate the results are once the data is analyzed. This chapter describes methods to determine sample size for vehicle intercept and license plate follow-up surveys. In addition, the license plate matching technique was simulated at varying capture rates and the vehicle tracing technique was simulated at varying probe samples to determine the effect on the overall accuracy of the estimation. The simulation was conducted using Microsoft Excel. The details of the simulation for each technique will be discussed in greater detail below.

\subsection{License Plate Matching Technique}

In the literature review, only one source (Schaefer, 1988) was found that explicitly defined the number of license plates that should be recorded at a roadside station for the license plate matching technique for the purpose of estimating the proportion of traffic at one station that has a particular destination. The equation is simply the required sample size $n$ to estimate a proportion $p$, given a particular level of confidence $z$ and error $e$. This equation is $n=p q z^{2} / e^{2}$, where $q=1-p$.

If an estimate of the proportion $p$ cannot be determined, the most conservative approach is to set $p=0.5$. The sample size $n$ for $95 \%$ confidence ( $z=1.96$, two-tailed) and $5 \%$ error is $n=(0.5)^{*}(1-0.5)^{*}\left(1.96^{2}\right) /\left(0.05^{2}\right)=384$ license plate observations.

While the equation is simple, it does not consider the volume of traffic from which the license plates are being sampled. As the traffic volume increases, the capture rate usually decreases, which reduces the number of matches obtained in the sample. Then, in expanding the sample to the population, the estimate for the proportion (and corresponding number of vehicles) can have high variability and error. 
For example, consider the situation in which the actual traffic volume at Station 1 and Station 2 is 10,000 vehicles, and the true proportion $p$ equals 0.25 , which means the total number of vehicles that pass both Stations 1 and 2 is $2,500\left(0.25^{*} 10,000\right)$. By capturing 384 vehicles at each station (3.8\% capture rate), a number of matches will be obtained. This situation was simulated 30 times, in which the number of matches obtained in the sample ranged from 0 to 9 . These samples were then expanded to estimate the actual proportion $p$ and number of vehicle passing both stations. While the average $p$ of these estimates was $25 \%$, the standard deviation was $14.5 \%(1,454$ vehicles). The estimates ranged from $0 \%$ (0 vehicles) to $61 \%(6,104$ vehicles), which is a range of $-2,500$ to 3,604 vehicles.

This example illustrates the variability that can be encountered in the license plate matching technique and the importance of collecting as many license plates as possible.

Even under the best circumstances, however, it is unlikely that all license plates on a particular road can or will be recorded during an OD study. In the matching technique, the license plate must be recorded correctly two separate times at two separate locations for that OD pair to be recognized. Because of this, missing license plates has the potential to introduce a significant amount of error. Therefore, it was desired to simulate various scenarios to determine how the quantity of the data recorded influences the accuracy of the projected results.

\subsubsection{Simulation}

As a starting point for this analysis, assume there are two recording stations (Station 1 and Station 2). A total of 1000 vehicles, each with a unique identifier (license plate), pass by each station. Assume that $25 \%$ of all vehicles (250 vehicles) that pass Station 1 also pass Station 2. The true proportion $p$ between stations is $25 \%$. This percentage and number of vehicles are the values sought during an OD study. If $100 \%$ of vehicles passing by both stations are captured, and all of the license plates at both stations are recorded correctly, 250 matches would be found between the stations and there would be zero error. However, this is an ideal situation and highly unlikely.

Knowing that the capture rates are never $100 \%$, the capture rates were varied at each station. Vehicles were randomly selected from each station to simulate the capture rate. The two stations were then compared and the number of matches between the 
stations was noted. Because it is assumed that there were no recording errors, the number of matches was divided by the capture rates at both stations to estimate the total number of trips between the two stations during the study period.

For example, assume again that a total of 1,000 vehicles pass both Station 1 and Station 2, and that $25 \%$ of vehicles passing Station 1 also pass Station 2. Now assume that $75 \%$ of vehicles passing Station 1 and $50 \%$ of vehicles passing Station 2 have their license plates captured in the field. By comparing the capture lists from each station (which were randomly selected from the full lists of all vehicles passing each station), the number of matches is found, then divided by $0.375\left(75 \%{ }^{*} 50 \%\right)$ to estimate the proportion and total number of trips between the two stations. The estimated number of trips is compared to the actual number of trips (in this case, $25 \%$ of 1000 vehicles, or 250 vehicles), and the percent error between the estimated and actual trips is calculated. The process of randomly selecting the percent of vehicles captured from each station, estimating the total number of trips, and calculating the percent error was conducted 50 times for each true proportion and capture rates at both stations. The standard deviation of the percent error was then calculated for each set. For this particular combination, the standard deviation after 50 simulations was $7.2 \%$. This means that, with $95 \%$ confidence, the estimated number of trips for a true proportion of $25 \%$ is within $\pm 14.1 \%$ of the true number of trips.

\subsubsection{Varying Capture Rates, Proportions, and Traffic Volumes}

The simulations described above only account for one combination of station capture rates, proportions, and station traffic volumes. Therefore, these variables were changed at certain intervals to evaluate a wide range of possible combinations.

First, using the same traffic volumes of 1,000 vehicles at each station, a true proportion of $1 \%$ between stations, the capture rates at each station were varied from $50 \%, 75 \%$, and $95 \%$. The capture rates at each station were held equal for simplicity, although in reality, they are independent of one another. Because expanding the data is based on the product of the capture rates, however, relative capture rates have little or no effect on the estimation error. In the end, 50 simulations were run on each of the 3 combinations of capture rates. 
After these simulations, the true proportion $p$ between the stations was changed to $5 \%$. Then, 50 simulations of the 3 combinations of capture rates were repeated. This was also repeated for a true proportion of $25 \%$ and $50 \%$.

Finally, the traffic volumes at both stations were changed, during which 50 simulations were run on all combinations of true proportions and capture rates. The traffic volumes at the stations were changed to 5,000 and 10,000 vehicles.

\subsubsection{Results}

Figures $42-45$ below illustrate the change in error as the true proportion of traffic between stations changes. Figure 42 illustrates the error for a proportion of $1 \%$, and Figures 43 - 45 illustrate the error for proportions of $5 \%, 25 \%$, and $50 \%$, respectively. On each figure, the product of capture rates is shown on the x-axis, and each line represents a different traffic volume at the observation stations (assuming the traffic volume is the same at both stations for simplicity).

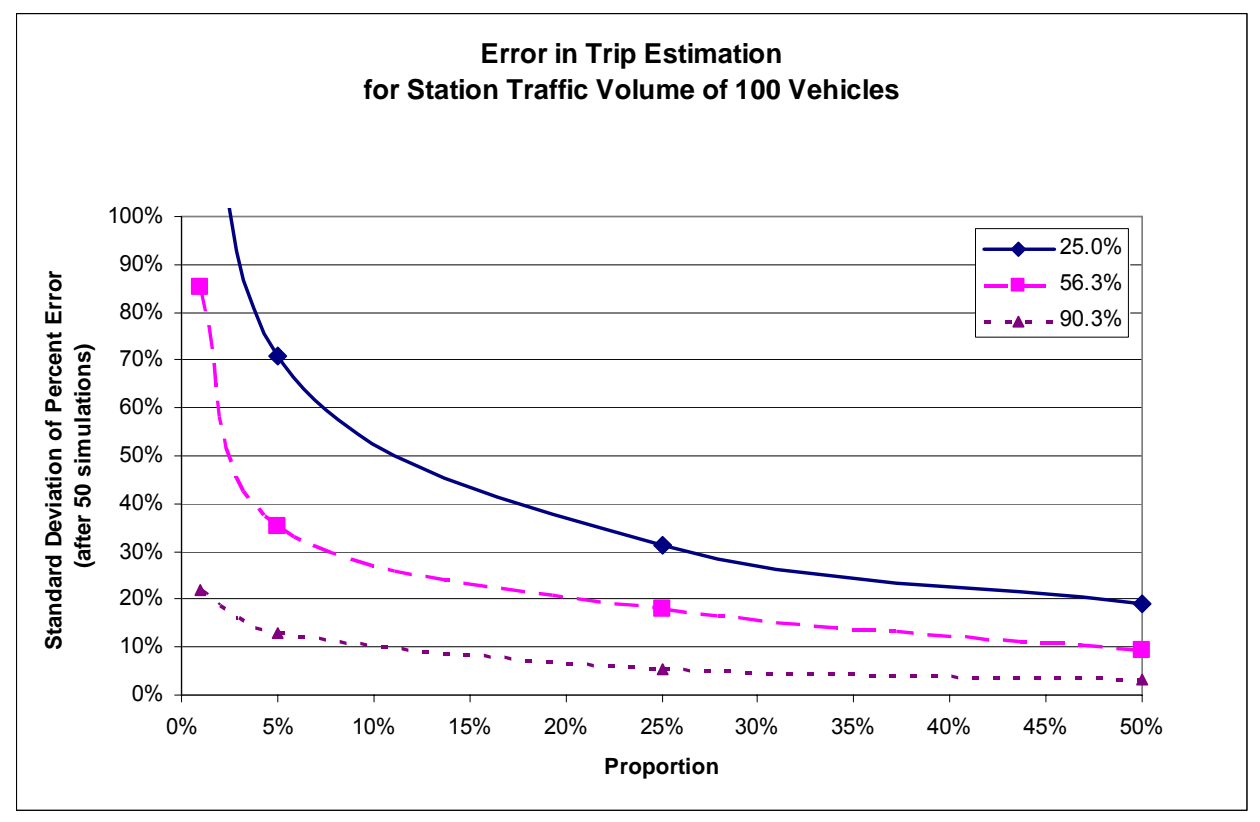

Figure 42: Error by Proportion for Traffic Volume of 100 Vehicles 


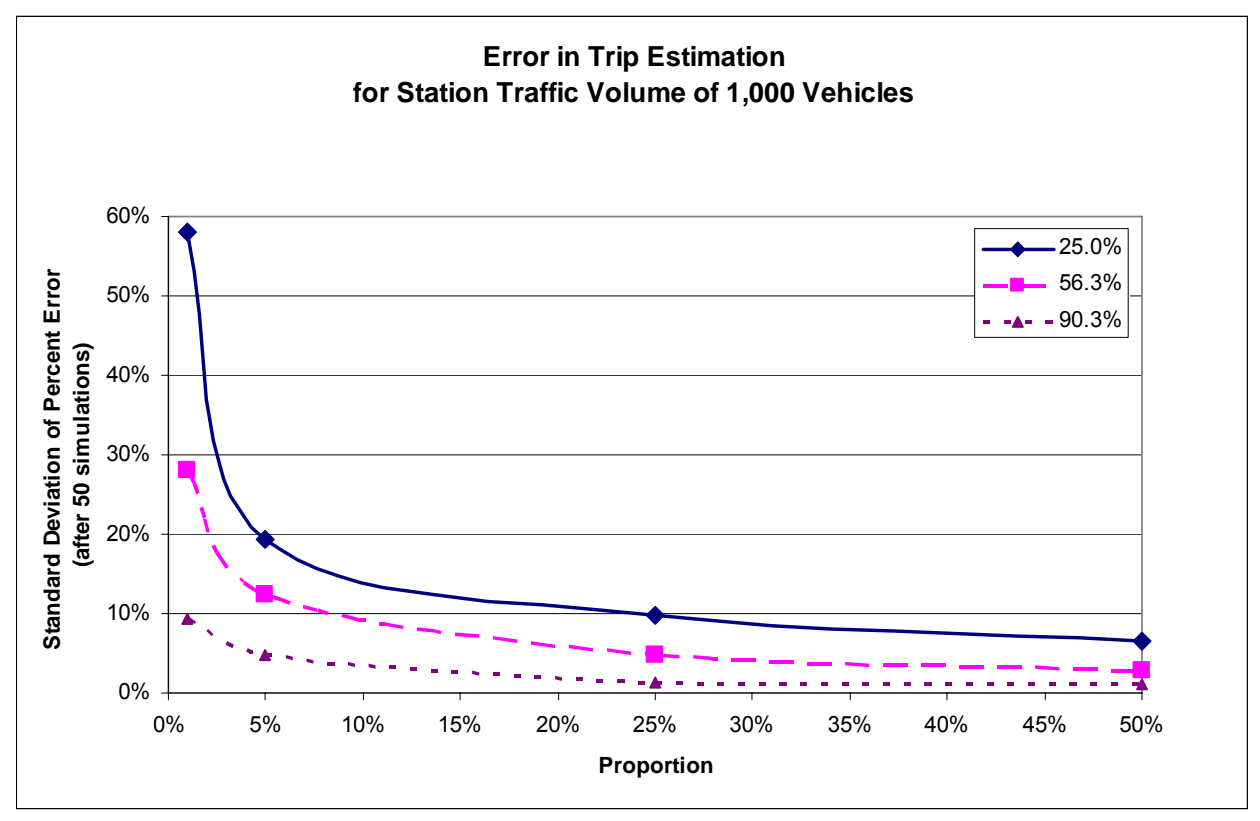

Figure 43: Error by Proportion for Traffic Volume of 1,000 Vehicles

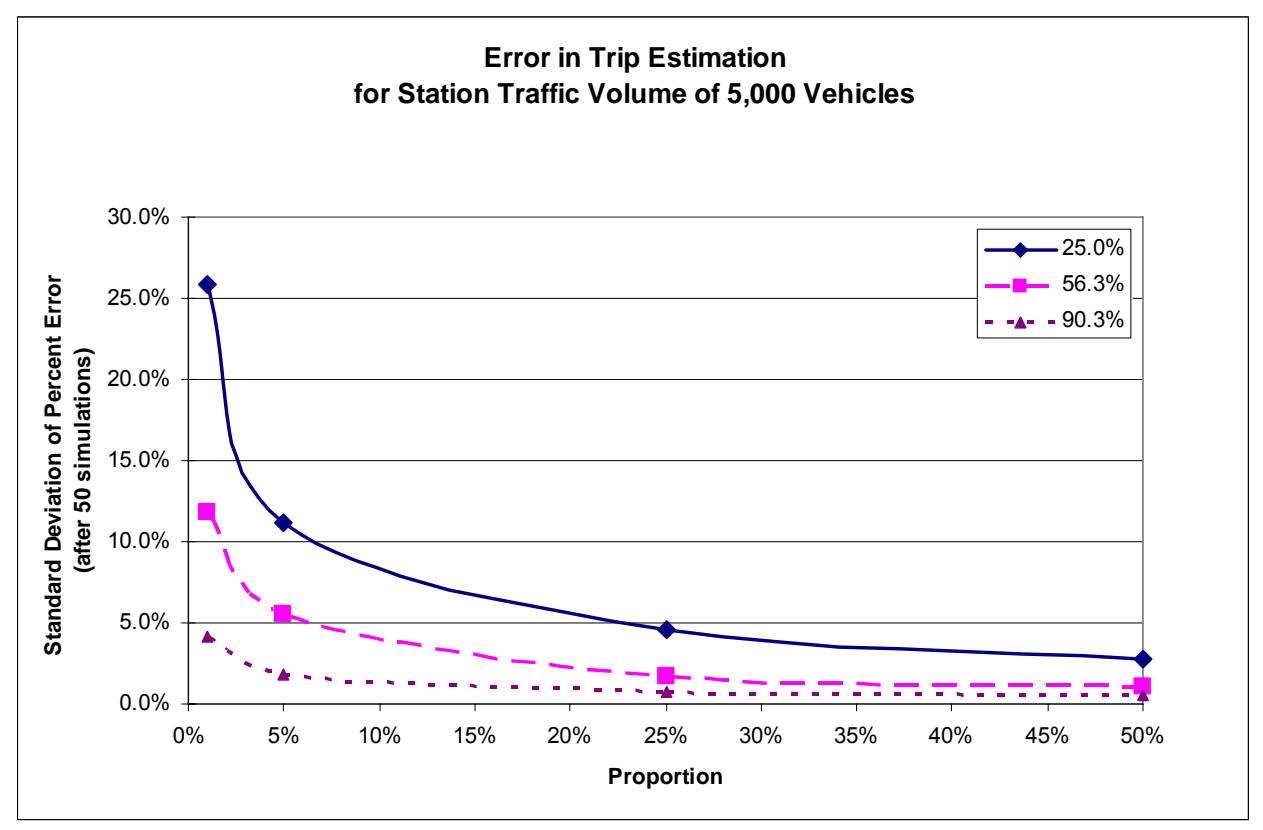

Figure 44: Error by Proportion for Traffic Volume of 5,000 Vehicles 


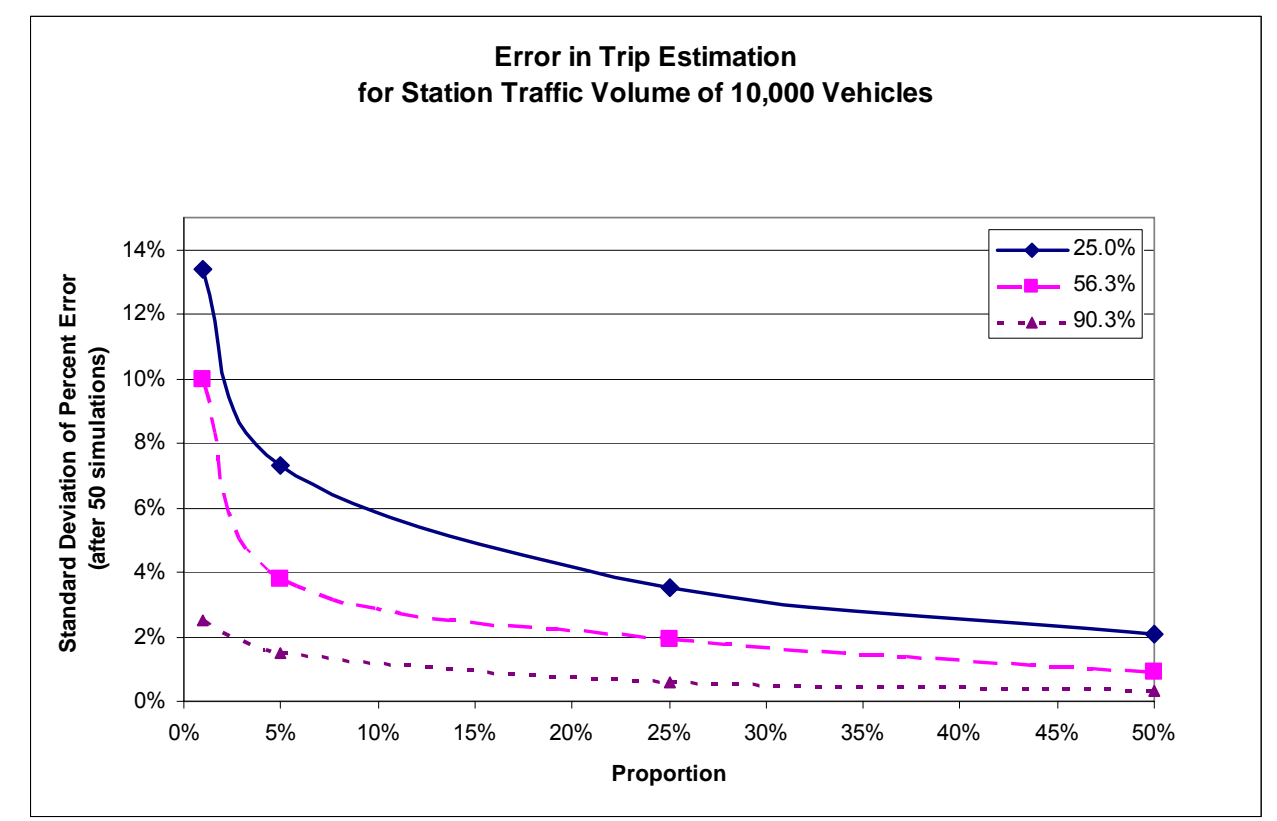

Figure 45: Error by Proportion for Traffic Volume of 10,000 Vehicles

Figures 42 - 45 above illustrate that, as the proportion between stations increases, the error in the estimated number of trips decreases. In any real situation, the proportion between any OD pair is seldom greater than $50 \%$. The points on the figures represent the proportions that were simulated. A product of capture rates of $25 \%$ is a $50 \%$ capture rate at each station. Likewise, $56.3 \%$ is the product of $75 \%$ capture at each station, and $90.3 \%$ is the product of $95 \%$ capture at each station. As stated earlier, the capture rates were held equal at each station, however, a product of $56.3 \%$ could also be obtained by station capture rates of $100 \%$ and $56.3 \%, 85 \%$ and $66 \%$, etc.

To describe the figures, assume, for example, $75 \%$ of license plates are recorded at the stations with traffic volumes of 5,000 vehicles, and 145 matches are found between the stations. The estimated total number of vehicles traveling between these two stations is determined by dividing the sample by the product of the capture rate. The estimated number of trips between the stations is $145 / 0.563=258$ vehicles. The estimated proportion of all traffic at Station 1 traveling to Station 2 is 258/5,000 $=0.051$. The standard deviation for a proportion of $5.1 \%$ is approximately $5.5 \%$ (from Figure 44 ). Therefore, the $95 \%$ confidence interval is $\pm 10.8 \%$ error in the true number of trips between the stations $(1.96 * 5.5 \%)$. Therefore, the true number of trips is between 230 and $286\left(258 \pm 258^{*} .108\right)$, and the true proportion is between $4.6 \%$ and $5.7 \%$. 
Figures 46 - 49 illustrate the same information presented in Figures 42 - 45 above. However, the $\mathrm{x}$-axis is traffic volume and each line represents a different capture rate product. Figure 46 illustrates errors for a proportion of 1\%, while Figures $47-49$ present errors for proportions of $5 \%, 25 \%$, and $50 \%$, respectively.

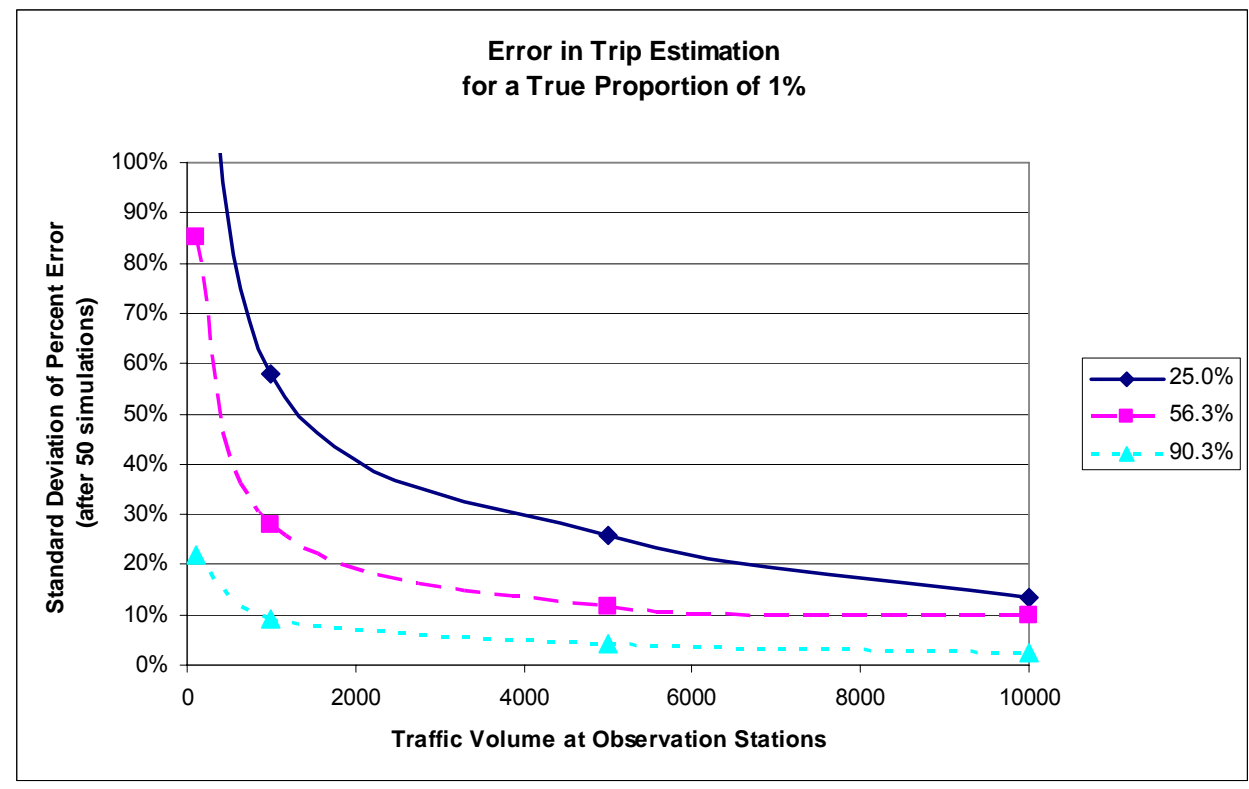

Figure 46: Error by Traffic Volume for a True Proportion of 1\%

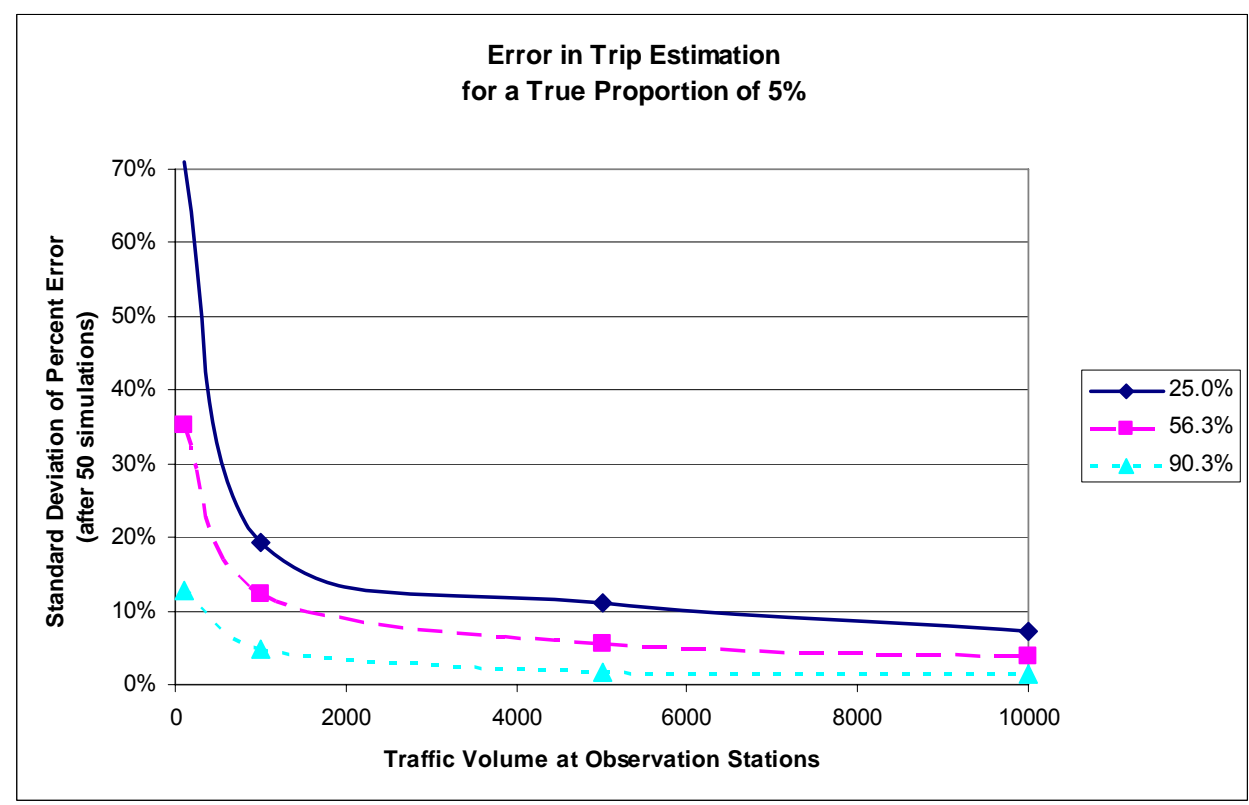

Figure 47: Error by Traffic Volume for a True Proportion of 5\% 


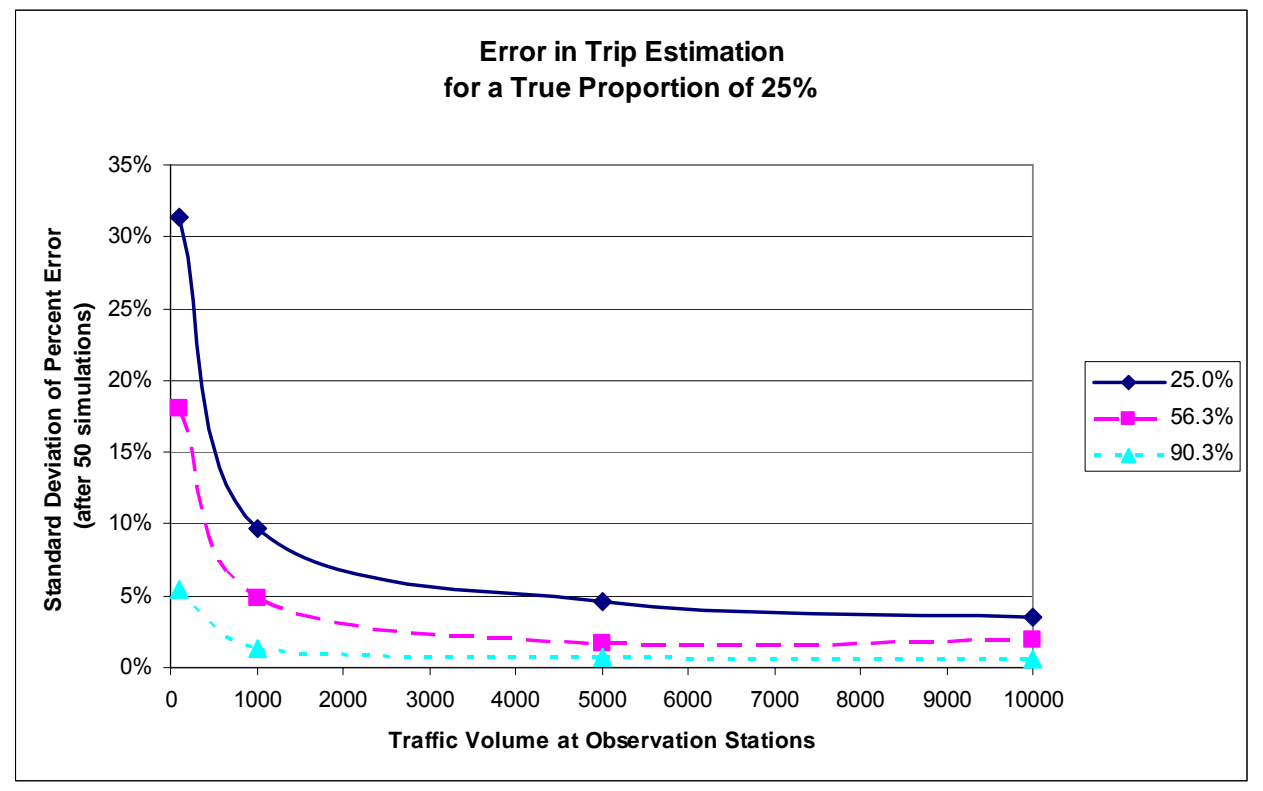

Figure 48: Error by Traffic Volume for a True Proportion of $25 \%$

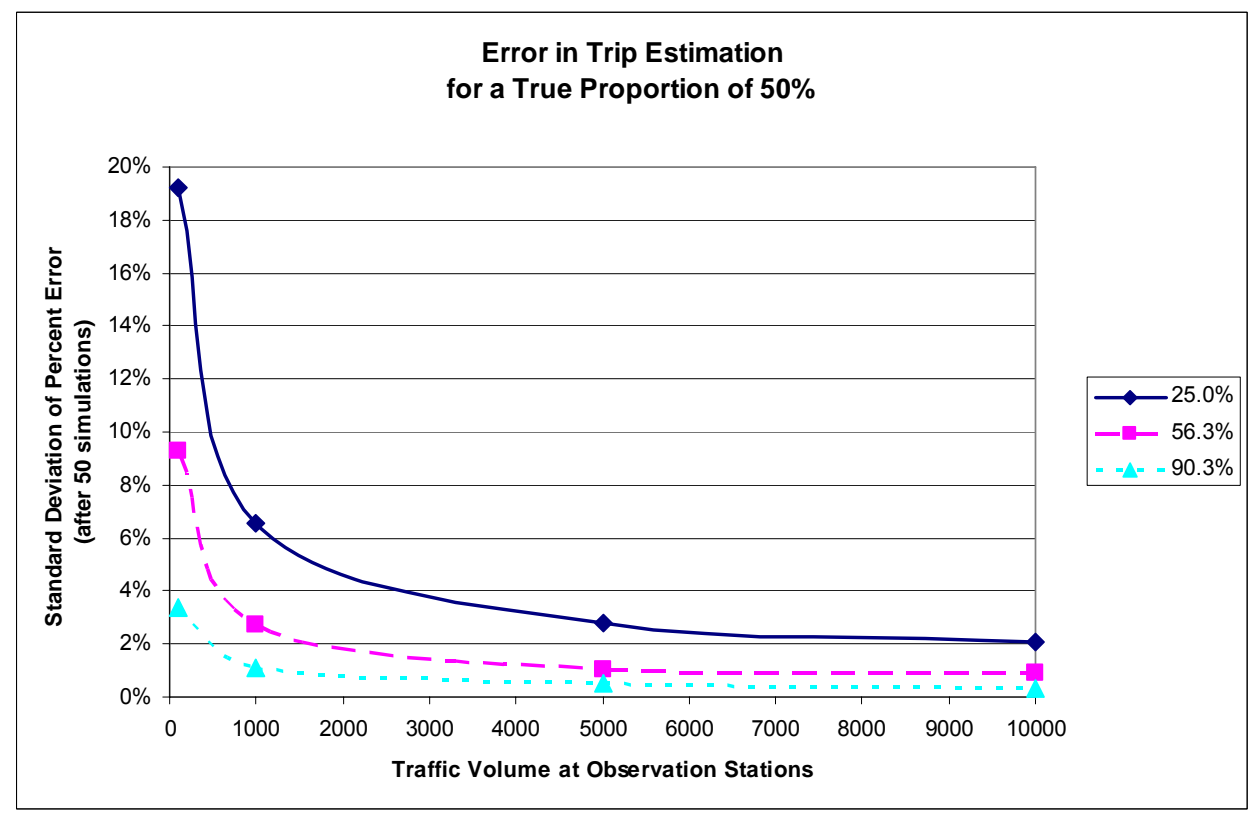

Figure 49: Error by Traffic Volume for a True Proportion of $50 \%$

Figure $46-49$ show that, for any given product of capture rates, the error decreases (at a decreasing rate) as traffic volumes increase. For example, Figure 49 shows that a $90.3 \%$ capture rate product has approximately the same accuracy when 
sampled on a roadway with 5,000 vehicles and 10,000 vehicles. The absolute number of vehicles sampled, however, is much higher for the higher volume road.

Figures $50-53$ illustrate the percent error in the estimated trips for station traffic volumes of $100,1,000,5,000$, and 10,000 vehicles, respectively.

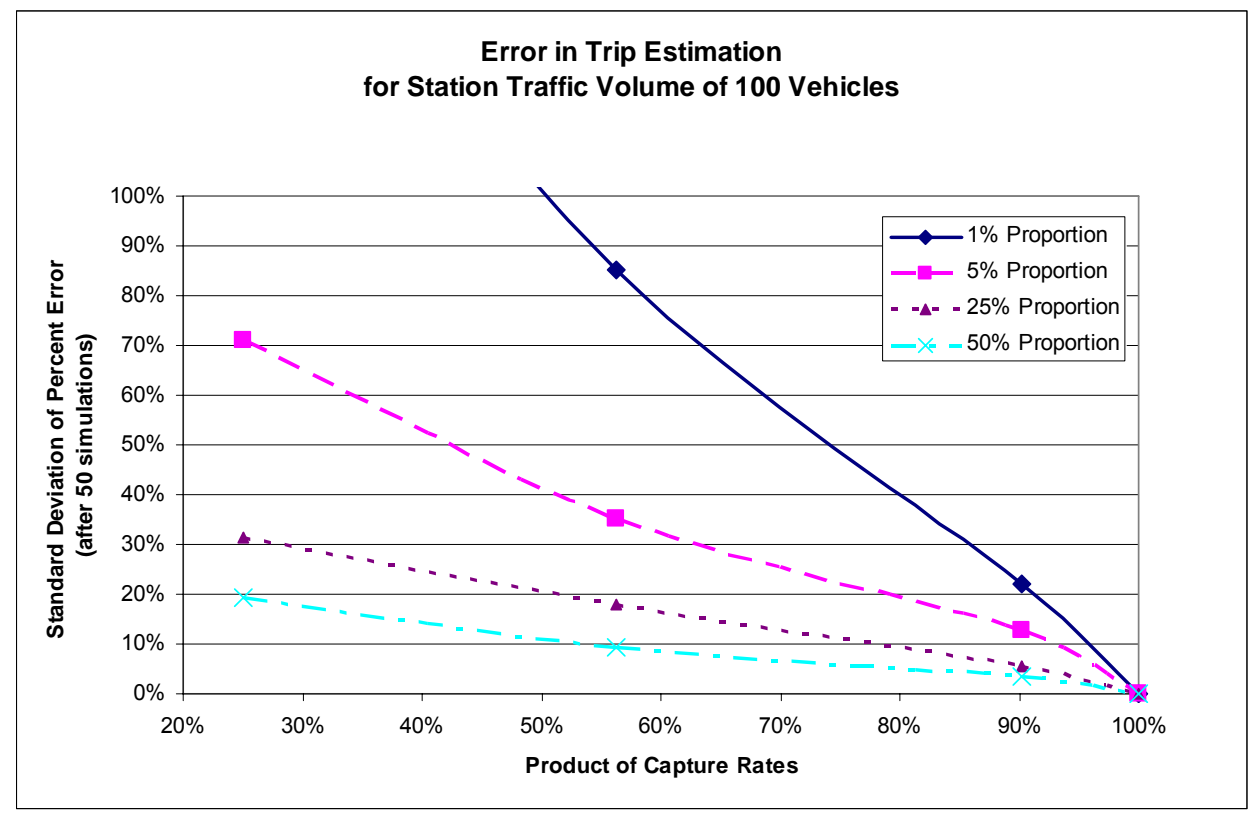

Figure 50: Error by Capture Rate Product for Traffic Volume of 100 Vehicles

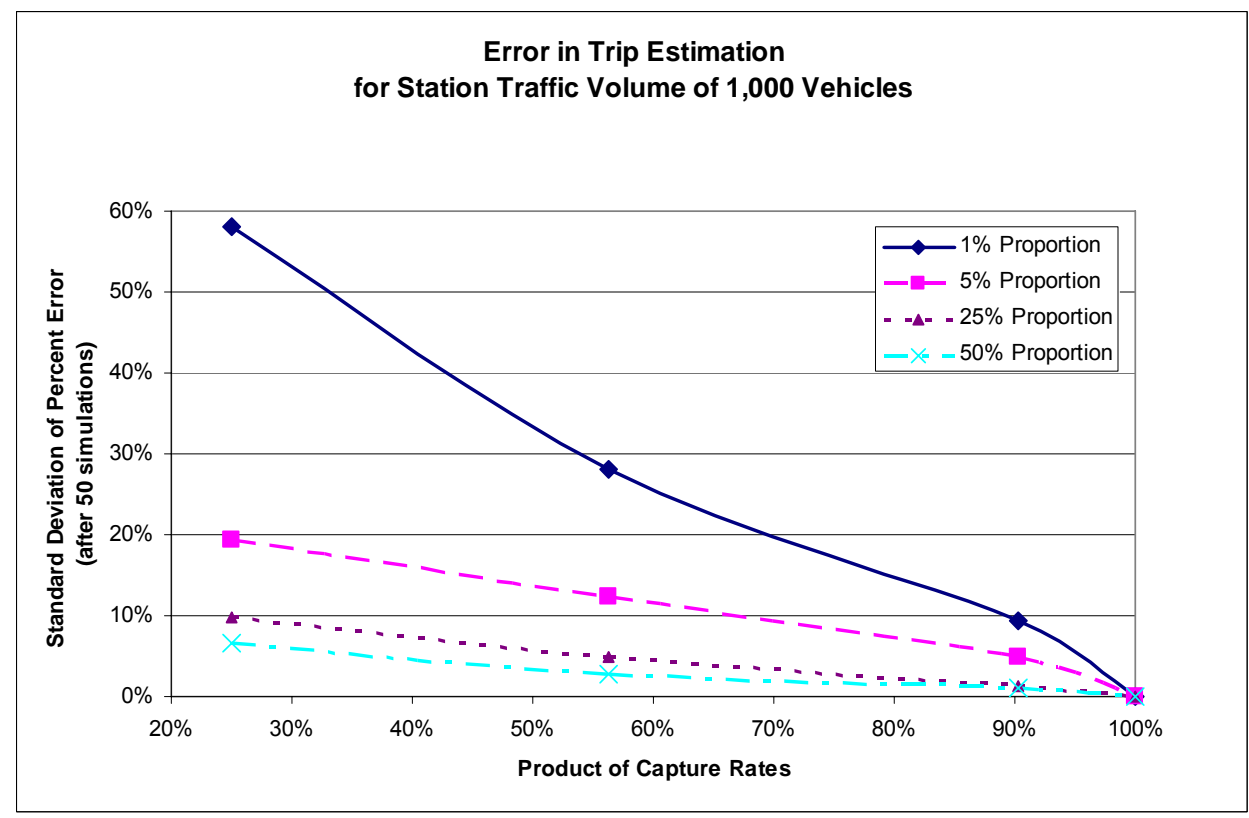

Figure 51: Error by Capture Rate Product for Traffic Volume of 1,000 Vehicles 


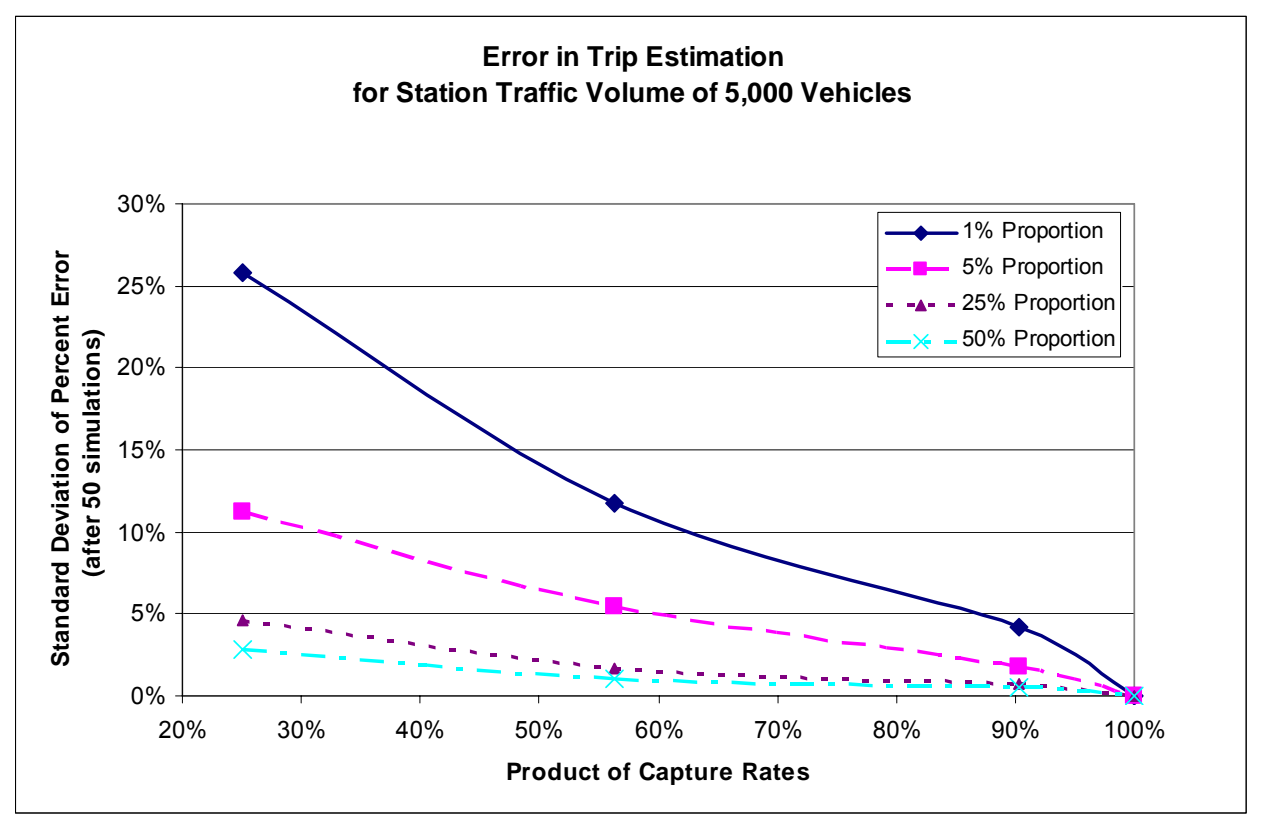

Figure 52: Error by Capture Rate Product for Traffic Volume of 5,000 Vehicles

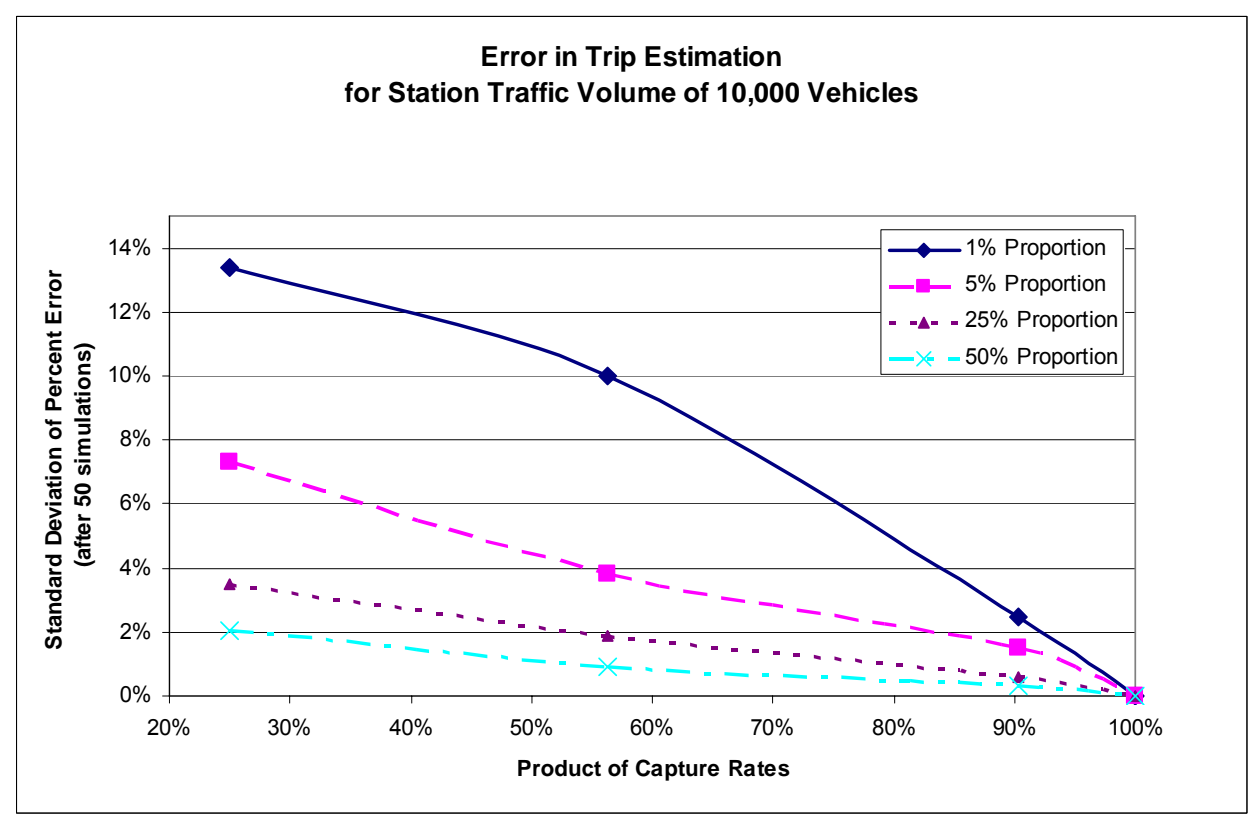

Figure 53: Error by Capture Rate Product for Traffic Volume of 10,000 Vehicles

It can be seen in Figure 50 that, for any given proportion, the percent error decreases as the product of capture rates increase. This is because, on average, the sample will contain less variability in the number of matches obtained, and the number of matches is not expanded as much. Table 17 summarizes the data used to build Figures $42-53$. 
Table 17: Standard Deviation of Percent Error in Trip Estimations

\begin{tabular}{|c|c|c|c|c|c|c|c|c|c|}
\hline \multicolumn{5}{|c|}{ 1\% Proportion } & \multicolumn{5}{|c|}{$5 \%$ Proportion } \\
\hline \multirow{2}{*}{$\begin{array}{c}\text { CR } \\
\text { Prod. }\end{array}$} & \multicolumn{4}{|c|}{ Traffic Volume } & \multirow{2}{*}{$\begin{array}{c}\text { CR } \\
\text { Prod. } \\
\end{array}$} & \multicolumn{4}{|c|}{ Traffic Volume } \\
\hline & 100 & 1000 & 5000 & 10000 & & 100 & 1000 & 5000 & 10000 \\
\hline $25.0 \%$ & $177.2 \%$ & $58.1 \%$ & $25.8 \%$ & $13.4 \%$ & $25.0 \%$ & $70.9 \%$ & $19.4 \%$ & $11.2 \%$ & $7.3 \%$ \\
\hline $56.3 \%$ & $85.1 \%$ & $28.0 \%$ & $11.8 \%$ & $10.0 \%$ & $56.3 \%$ & $35.2 \%$ & $12.4 \%$ & $5.5 \%$ & $3.8 \%$ \\
\hline $90.3 \%$ & $21.9 \%$ & $9.4 \%$ & $4.2 \%$ & $2.5 \%$ & $90.3 \%$ & $12.8 \%$ & $4.8 \%$ & $1.8 \%$ & $1.5 \%$ \\
\hline $100 \%$ & $0 \%$ & $0 \%$ & $0 \%$ & $0 \%$ & $100 \%$ & $0 \%$ & $0 \%$ & $0 \%$ & $0 \%$ \\
\hline \multicolumn{5}{|c|}{$25 \%$ Proportion } & \multicolumn{5}{|c|}{$50 \%$ Proportion } \\
\hline \multirow{2}{*}{$\begin{array}{c}\text { CR } \\
\text { Prod. }\end{array}$} & \multicolumn{4}{|c|}{ Traffic Volume } & \multirow{2}{*}{$\begin{array}{c}\text { CR } \\
\text { Prod. } \\
\end{array}$} & \multicolumn{4}{|c|}{ Traffic Volume } \\
\hline & 100 & 1000 & 5000 & 10000 & & 100 & 1000 & 5000 & 10000 \\
\hline $25.0 \%$ & $31.4 \%$ & $9.7 \%$ & $4.6 \%$ & $3.5 \%$ & $25.0 \%$ & $19.2 \%$ & $6.5 \%$ & $2.8 \%$ & $2.1 \%$ \\
\hline $56.3 \%$ & $18.1 \%$ & $4.8 \%$ & $1.7 \%$ & $1.9 \%$ & $56.3 \%$ & $9.3 \%$ & $2.7 \%$ & $1.0 \%$ & $0.9 \%$ \\
\hline $90.3 \%$ & $5.5 \%$ & $1.3 \%$ & $0.7 \%$ & $0.6 \%$ & $90.3 \%$ & $3.4 \%$ & $1.1 \%$ & $0.5 \%$ & $0.3 \%$ \\
\hline $100 \%$ & $0 \%$ & $0 \%$ & $0 \%$ & $0 \%$ & $100 \%$ & $0 \%$ & $0 \%$ & $0 \%$ & $0 \%$ \\
\hline
\end{tabular}

The capture rates become a bigger issue when the OD volumes of a subset of vehicles, such as trucks, are being determined, because the traffic volume (number of trucks observed) during the study period may be low, which increases the error in the estimation. In this case, it is important that high capture rates are achieved. Because of the factors cited in Chapter 6 and the lower accuracy mentioned above, the video method is recommended for recording license plates in truck OD studies.

\subsection{Vehicle Intercept and License Plate Follow-Up Survey Techniques}

The accuracy of vehicle intercept surveys and license plate follow-up surveys for a given station depends on the number of completed surveys at a particular station. For vehicle intercept surveys, a higher percentage of surveys (generally $>90 \%$ ) will be completed in the roadside interview method than with the postcard questionnaire method. In order to obtain the same amount of information as the roadside interview method, the postcard questionnaires will have to be distributed to more vehicles. In terms of response rates, postcard questionnaires are much more similar to a license plate follow-up survey, because the surveys are completed at the will of the driver, generally $15 \%-30 \%$, depending on the length and format of the questionnaire (Virkud, 1995). For license plate follow-up surveys, full license plate numbers have to be 
captured in the field and matched with an address at the BMV, which increases the number of license plates that must be recorded.

While the Travel Survey Manual (TMIP, 1996) provides sample size equations for household travel surveys, it does not explicitly provide any details for determining sample sizes for vehicle intercept surveys. In searching the literature for sample size equations, two sources were found, as discussed below.

The first equation was found in a document entitled "Traffic Surveys by Roadside Interview" (The Highways Agency, 1992) published by several agencies in the United Kingdom. The equation is:

$$
n=\frac{p q N^{3}}{\left[\left(\frac{E}{Z}\right)^{2}(N-1)+p q N^{2}\right]}
$$

$n=$ number of completed surveys required

$p=$ estimated proportion of total traffic at the station with a particular destination

$q=1-p$

$E=$ accuracy or absolute error (percent error * $N$ )

$N=$ traffic volume at survey station

$Z=$ normal variate for specified level of confidence (eg. 1.96 for $95 \%$ confidence)

Stokes et al. (1989) use another equation proposed by Hajek (1977) that

provides the sampling rate required to develop external-external trip tables based on the estimated proportion of traffic for a particular OD pair, the desired accuracy and confidence level, and the volume of traffic at the station. The equation is shown below:

$$
\begin{aligned}
& r=\frac{Z^{2} p q}{\left[(N-1) W^{2}+\left(Z^{2} p q\right)\right]} \\
& r=\text { sample rate } \\
& p=\text { estimated proportion of total traffic at the station with a particular destination } \\
& q=1-p \\
& \left.W=\text { desired accuracy (percent error }{ }^{*} p\right) \\
& N=\text { traffic volume at the survey station } \\
& Z=\text { normal variate for specified level of confidence }
\end{aligned}
$$


The total number of required completed surveys $n$ is simply the sample rate $r$ times the traffic volume $N$.

While most reports on vehicle intercept and license plate follow-up surveys do not explicitly state how the sample size was determined, many specify the sample rate at which surveys were administered. For a specific confidence interval and error, the sample rate increases as the true proportion $p$ decreases. In addition, the sample rate decreases as the traffic volume increases (the absolute number of samples increases, however). This follows the results obtained from simulating the license plate matching technique in the previous section in that, as the true proportion between two stations decreases, the required capture rate increases (to estimate at the same level of confidence and error). Therefore, the Hajeck's equation is recommended for determining sample sizes for vehicle intercept and license plate follow-up surveys.

The sample size $n$ obtained from the Hajek is actually the required number of completed surveys. Therefore, depending upon the method used, this number will have to be increased to account for non-response of surveys and errors in the data collection process.

For roadside interviews, a small percentage of drivers (generally $<10 \%$ ) will refuse to participate in the survey. Therefore, the total number of vehicles stopped for an interview should be $n / 0.9$.

For the postcard questionnaire handout method, generally $15 \%$ - 30\% response can be expected. Prior to the actual study, tests should be conducted to more accurately determine the response rate. Therefore, the number of postcards handed out should be approximately n/0.15 (Virkud, 1995).

Finally, for the license plate follow-up survey technique, the number of license plates recorded should again account for a $15 \%$ - 30\% response rate, but also for errors in recording license plates in the field (those that will not match with the BMV database) which is assumed to be no greater than $10 \%$ based on the error analysis conducted in Chapter 6 . Therefore, the total number of license plates recorded should be $n /\left[0.15^{*} 0.90\right]$.

\subsection{Vehicle Tracing Technique}

While the vehicle tracing technique has not generally been used in practice on a large scale, an analysis was conducted to determine the accuracy of the estimated OD 
matrix changes with varying degrees of sampling cell phones or GPS systems (probe vehicles).

\subsubsection{Simulation}

This analysis assumes that the data obtained on probe vehicles was perfect, meaning that the origin and destination zones in which a trip began and ended are known.

To start, a simple $4 \times 4$ zone OD matrix was constructed, with each OD pair containing 100 trips. Therefore, the total number of trips in the matrix was 1600 . In reality, these zones could represent actual traffic analysis zones or entry/exit nodes on the cordon line of a study area. A vehicle identifier was created for each vehicle that contained the origin and destination. From the 1600 vehicles, a sample of vehicles was chosen randomly, from which the probe vehicle OD matrix was created. The probe vehicle OD matrix was expanded globally to determine an estimated OD matrix. This estimated OD matrix was compared to the true OD matrix by calculating the root mean square error (RMSE) and percent root mean square error (PRMSE). Tables 18 - 20 illustrate this process.

Table 18: Actual OD Matrix (OD1)

\begin{tabular}{|c|c|c|c|c|c|}
\hline OD1 & $\mathbf{1}$ & $\mathbf{2}$ & $\mathbf{3}$ & $\mathbf{4}$ & Total \\
\hline $\mathbf{1}$ & 100 & 100 & 100 & 100 & 400 \\
\hline $\mathbf{2}$ & 100 & 100 & 100 & 100 & 400 \\
\hline $\mathbf{3}$ & 100 & 100 & 100 & 100 & 400 \\
\hline $\mathbf{4}$ & 100 & 100 & 100 & 100 & 400 \\
\hline Total & 400 & 400 & 400 & 400 & 1600 \\
\hline
\end{tabular}

Table 19: Sample Probe Vehicle OD Matrix*

\begin{tabular}{|c|c|c|c|c|c|}
\hline $\mathbf{5 0 \%}$ & $\mathbf{1}$ & $\mathbf{2}$ & $\mathbf{3}$ & $\mathbf{4}$ & Total \\
\hline $\mathbf{1}$ & 53 & 50 & 48 & 51 & 202 \\
\hline $\mathbf{2}$ & 59 & 51 & 49 & 45 & 204 \\
\hline $\mathbf{3}$ & 45 & 53 & 51 & 51 & 200 \\
\hline $\mathbf{4}$ & 46 & 53 & 46 & 49 & 194 \\
\hline Total & 203 & 207 & 194 & 196 & 800 \\
\hline
\end{tabular}

*In this case, the probe vehicle matrix is based on a $50 \%$ sample. 
Table 20: Estimated OD Matrix (Expanded Probe Vehicle Matrix)

\begin{tabular}{|c|c|c|c|c|c|}
\hline $\mathbf{1 0 0} \mathbf{\prime}^{\prime}$ & $\mathbf{1}$ & $\mathbf{2}$ & $\mathbf{3}$ & $\mathbf{4}$ & Total \\
\hline $\mathbf{1}$ & 106 & 100 & 96 & 102 & 404 \\
\hline $\mathbf{2}$ & 118 & 102 & 98 & 90 & 408 \\
\hline $\mathbf{3}$ & 90 & 106 & 102 & 102 & 400 \\
\hline $\mathbf{4}$ & 92 & 106 & 92 & 98 & 388 \\
\hline Total & 406 & 414 & 388 & 392 & 1600 \\
\hline
\end{tabular}

The RMSE and PRMSE for the estimated OD matrix for Table 18 is 7.1 trips and $7.1 \%$, respectively. The reason the PRMSE is calculated is to compare other OD matrices that have a different total number of trips in the matrix. In addition, this matrix was also sampled at different rates. Table 19 shows the probe vehicle OD matrix based on a $50 \%$ sample (800 vehicles). The same matrix was estimated from probe vehicles based on $1 \%, 5 \%, 25 \%, 50 \%, 75 \%, 95 \%$, and $99 \%$ sample rates as well.

The process of obtaining the probe vehicle OD matrix was repeated 50 times for each sampling rate. The mean of the RMSE and PRMSE were then calculated from the 50 simulations. Figure 54 illustrates the results of this analysis.

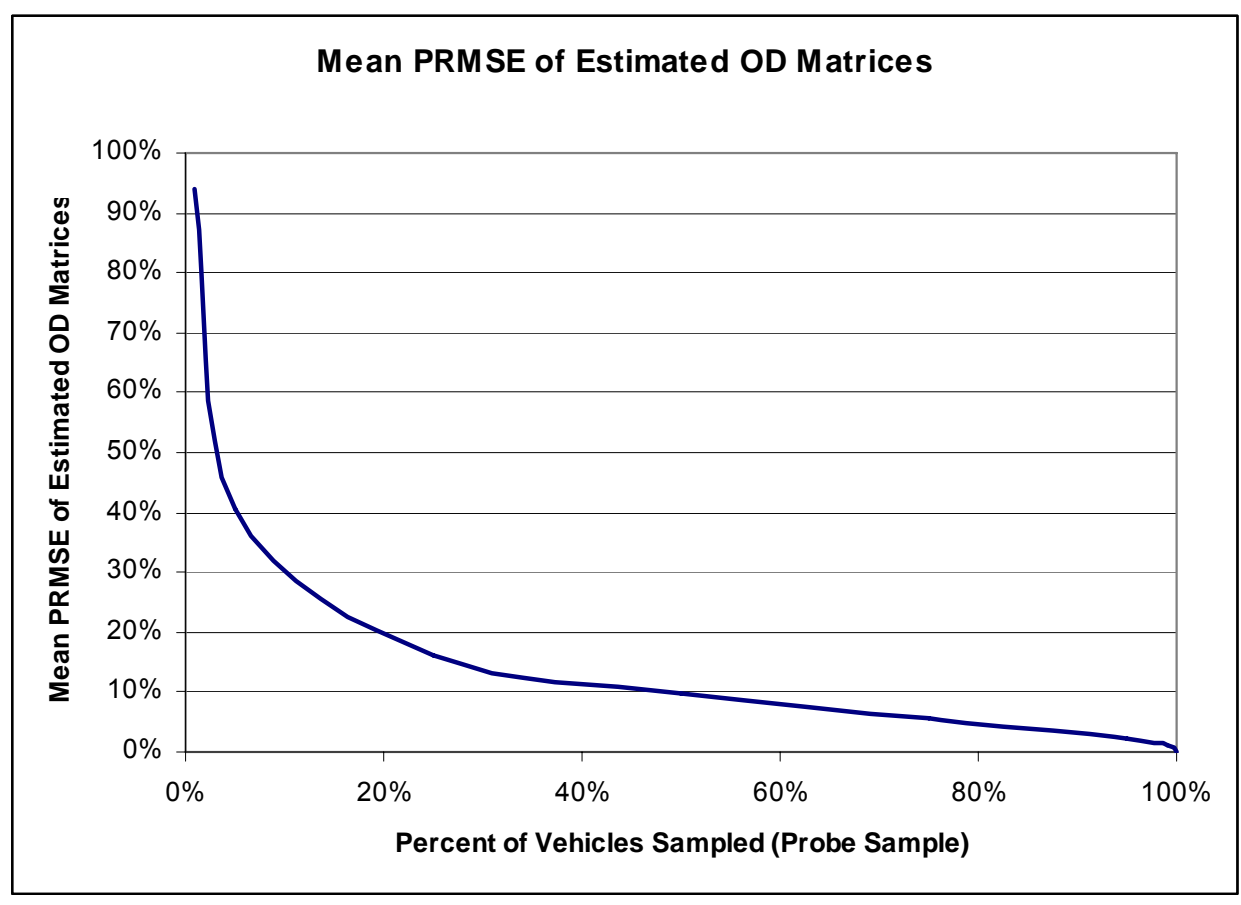

Figure 54: PRMSE of Estimated OD Matrices based on OD1 (Table 18) 
Figure 54 illustrates that as the number of probe vehicles increase, the PRMSE decreases, which is expected. At $50 \%$ sampling, the mean PRMSE drops to approximately $10 \%$.

Because this figure was developed only for a 16-cell matrix with an equal number of trips per OD pair, it was desired to see how the error changed as the number of zones of the true OD matrix changed or as the number of trips in a 16-cell matrix shifted so that they were not uniformly distributed to each OD pair. This is discussed in the next section.

\subsubsection{Results}

The same procedure was conducted on other types of OD matrices to determine the relative change in the accuracy of the estimated OD matrix. Tables $21-24$ illustrate the other types of OD matrices that were randomly sampled.

Table 21: 4x4 1600-trip Matrix (OD2) without Uniformly-Distributed Cells

\begin{tabular}{|c|c|c|c|c|c|}
\hline OD2 & $\mathbf{1}$ & $\mathbf{2}$ & $\mathbf{3}$ & $\mathbf{4}$ & Total \\
\hline $\mathbf{1}$ & 0 & 0 & 0 & 0 & 0 \\
\hline $\mathbf{2}$ & 7 & 7 & 7 & 7 & 28 \\
\hline $\mathbf{3}$ & 49 & 49 & 49 & 49 & 196 \\
\hline $\mathbf{4}$ & 344 & 344 & 344 & 344 & 1376 \\
\hline Total & 400 & 400 & 400 & 400 & 1600 \\
\hline
\end{tabular}

Table 22: 4x4 1600-trip Matrix (OD3) without Uniformly-Distributed Cells or Zeros

\begin{tabular}{|c|c|c|c|c|c|}
\hline OD3 & $\mathbf{1}$ & $\mathbf{2}$ & $\mathbf{3}$ & $\mathbf{4}$ & Total \\
\hline $\mathbf{1}$ & $\mathbf{7}$ & 7 & 7 & 7 & 28 \\
\hline $\mathbf{2}$ & 7 & 7 & 7 & 7 & 28 \\
\hline $\mathbf{3}$ & 42 & 42 & 42 & 42 & 168 \\
\hline $\mathbf{4}$ & 344 & 344 & 344 & 344 & 1376 \\
\hline Total & 400 & 400 & 400 & 400 & 1600 \\
\hline
\end{tabular}

Table 23: 4x4 16,000-trip Matrix (OD4) with Uniformly-Distributed Cells

\begin{tabular}{|c|c|c|c|c|c|}
\hline OD4 & $\mathbf{1}$ & $\mathbf{2}$ & $\mathbf{3}$ & $\mathbf{4}$ & Total \\
\hline $\mathbf{1}$ & 1,000 & 1,000 & 1,000 & 1,000 & 4,000 \\
\hline $\mathbf{2}$ & 1,000 & 1,000 & 1,000 & 1,000 & 4,000 \\
\hline $\mathbf{3}$ & 1,000 & 1,000 & 1,000 & 1,000 & 4,000 \\
\hline $\mathbf{4}$ & 1,000 & 1,000 & 1,000 & 1,000 & 4,000 \\
\hline Total & 4,000 & 4,000 & 4,000 & 4,000 & 16,000 \\
\hline
\end{tabular}


Table 24: 8x8 1600-trip Matrix (OD5) with Uniformly-Distributed Cells

\begin{tabular}{|c|c|c|c|c|c|c|c|c|c|}
\hline OD5 & $\mathbf{1}$ & $\mathbf{2}$ & $\mathbf{3}$ & $\mathbf{4}$ & $\mathbf{5}$ & $\mathbf{6}$ & $\mathbf{7}$ & $\mathbf{8}$ & Total \\
\hline $\mathbf{1}$ & 25 & 25 & 25 & 25 & 25 & 25 & 25 & 25 & 200 \\
\hline $\mathbf{2}$ & 25 & 25 & 25 & 25 & 25 & 25 & 25 & 25 & 200 \\
\hline $\mathbf{3}$ & 25 & 25 & 25 & 25 & 25 & 25 & 25 & 25 & 200 \\
\hline $\mathbf{4}$ & 25 & 25 & 25 & 25 & 25 & 25 & 25 & 25 & 200 \\
\hline $\mathbf{5}$ & 25 & 25 & 25 & 25 & 25 & 25 & 25 & 25 & 200 \\
\hline $\mathbf{6}$ & 25 & 25 & 25 & 25 & 25 & 25 & 25 & 25 & 200 \\
\hline $\mathbf{7}$ & 25 & 25 & 25 & 25 & 25 & 25 & 25 & 25 & 200 \\
\hline $\mathbf{8}$ & 25 & 25 & 25 & 25 & 25 & 25 & 25 & 25 & 200 \\
\hline Total & 200 & 200 & 200 & 200 & 200 & 200 & 200 & 200 & 1600 \\
\hline
\end{tabular}

After the probe vehicle matrix was created based on the varying degrees of sampling and then expanded to estimate the original OD matrix for each of the true OD matrices listed above, the mean PRMSE was calculated from 50 simulations and compared to mean of each of the other OD matrices to see how the accuracy differed. Figure 54 illustrates these results.

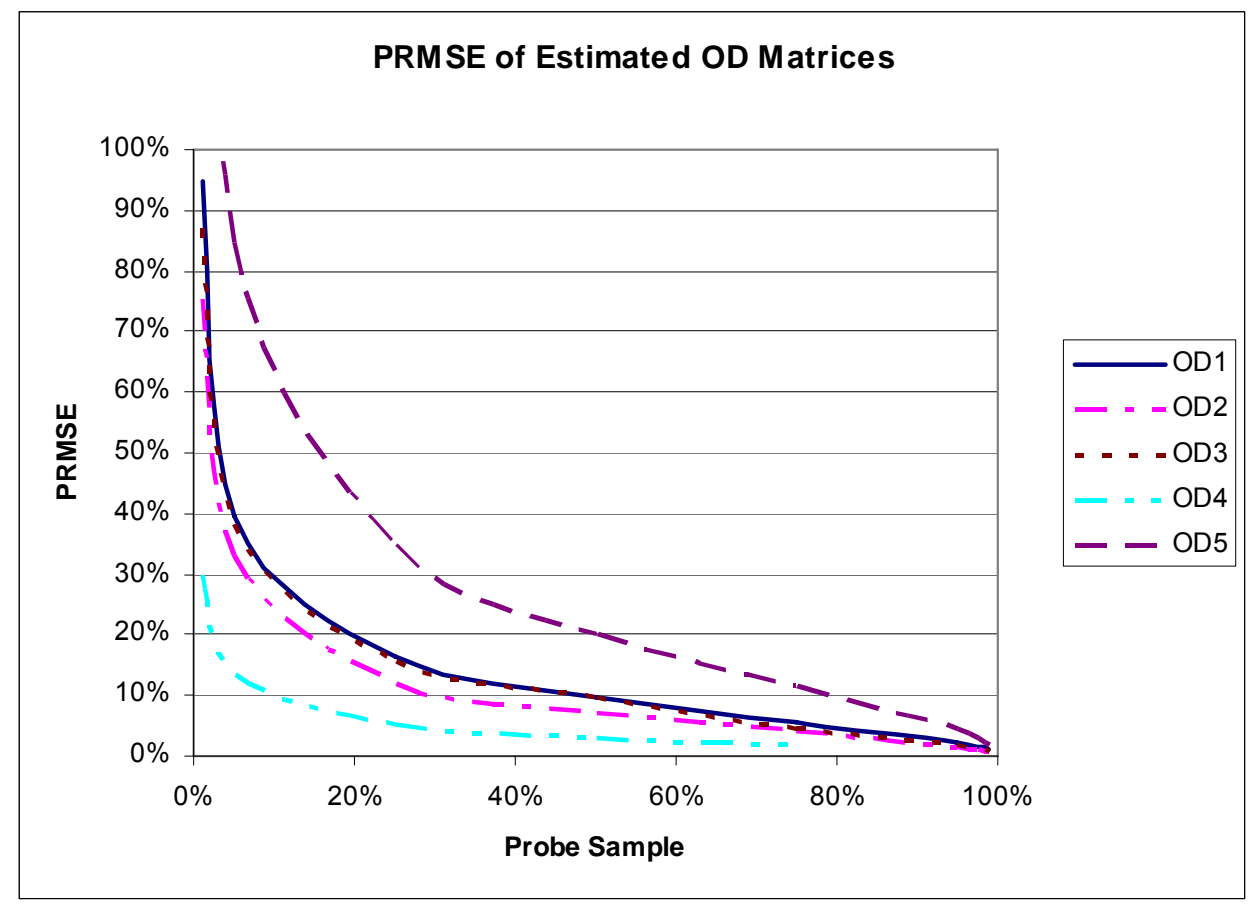

Figure 55: Mean PRMSE for Various OD Matrices

It can be seen in Figure 54 that OD5 (Table 24) has the highest error, and OD4 (Table 23) has the lowest error for any given probe sample. In addition, OD1 (Table 18) 
and OD3 (Table 22) have equal error. How can this be explained? It seems that, given a particular probe sample rate, the overall error for the OD matrix depends upon the average number of trips per non-zero OD pair. For example, OD1 and OD3 both have 100 trips per non-zero pair. OD2 (Table 21 ), which has slightly more (133 trips per nonzero pair), has slightly better overall accuracy at the same sampling rate. The same is true regardless of the number of zones in the matrix. OD5, which has 25 trips per nonzero pair, has far less accuracy. The opposite is true for OD4, which has 1,000 trips per non-zero OD pair.

The problem in practice is that, if given a set of data from probe vehicles, the size of the sample relative to the number of vehicles sought to be estimated in the OD matrix (the sampling rate) is unknown. Without the sampling rate, it is difficult to expand the probe vehicle matrix to the estimated OD matrix to obtain the number of trips per OD pair. The probe sampling rate can be approximated by determining the average ratio of traffic volumes from the sample to those of actual counts (such as tube counters) for a number of links in the study area. This analysis, however, serves as a general starting point for determining accuracy when estimating OD matrices from probe vehicle data such as cell phones and in-vehicle GPS systems. 


\section{CHAPTER 8 - GUIDELINES FOR SELECTING AN OD TECHNIQUE AND METHOD}

The purpose of this chapter is to combine elements of the previous chapters, the Travel Survey Manual, and other sources to assist a planner in determining the technique and method by which an OD study should be conducted based on the objectives of the study, cost constraints, desired accuracy of the results, and traffic conditions in the study area.

\subsection{Summary of Findings for License Plate Data Collection}

Before identifying and recommending general guidelines, the following four sections summarize the findings for license plate data collection as a result of the analyses conducted as part of this project.

1. Vehicle Speed and Capture Rate

In Section 6.3, the maximum vehicle speed for identifying license plates with half-sized (1.25") characters was determined for manual recording methods (clipboard, audio, and laptop) for various levels of visual acuity.

For example, a person with 20/20 visual acuity recording 4-characters off license plates from vehicles traveling $30 \mathrm{mph}$ would capture approximately $25 \%$ of license plates with half-sized characters (Figure 39).

Because Indiana issues many license plates with characters that are 1.25 " in height, the maximum speed at which nearly all license plates can be captured is relatively low for a person with normal visual acuity $(20 \mathrm{mph}$ for 4character recording and $10 \mathrm{mph}$ for full string recording). This maximum speed would increase if only 2.75 " characters were used on license plates (approximately $45 \mathrm{mph}$ for 4-character and $30 \mathrm{mph}$ for full string recording). 
For these reasons, it is recommended that video be used for recording license plates if the prescribed vehicle speed is exceeded.

\section{Vehicle Flow and Capture Rate}

In Section 6.4, the maximum vehicle flow for recording license plates was determined for the manual recording methods based on the amount of time required to record a license plate and the headways between vehicles. For example, if the audio method is being used on a roadway with uninterrupted flow of 350 vehicles per hour per lane, the capture rate of those vehicles is $\sim 85 \%$.

Of the three manual methods, audio can achieve the highest capture rate for a given flow rate. For flow rates above which capture rates cannot be achieved by the clipboard, audio, or laptop methods, the video method is recommended.

The values determined in Section 6.4 are for uninterrupted flow only. For interrupted flow (e.g., downstream from a traffic signal), vehicle headways will be much shorter (depending on the amount of queueing at the signal), so video is always recommended.

3. Issues with Audio Recorders and Digital Video Camcorders

Chapter 5 discusses many of the issues when recording license plates with audio or video equipment.

For audio, the primary issue is the quality of the recording (the clarity of the license plate records during playback). Generally, it is recommended that the highest recording quality be used. In addition, it was determined that cassette audio recorders are better than digital because they have external storage (tapes) that can be changed quickly and easily.

For video, there are many more issues to consider, such as set up location, shooting angle, exposure settings, magnification, and shutter speed. Section 5.8 describes all of these in greater detail.

When selecting video equipment, it is recommended that one camcorder be purchased or rented and that field tests be conducted prior to the actual study, in order to ensure that a particular camcorder model is adequate for recording license plates from the roadside. 


\section{Accuracy of the License Plate Matching Technique}

Section 7.1 evaluated scenarios with the license plate matching technique in which the traffic volume, proportion between stations (the percentage of vehicles observed at one station that also pass a second station), and capture rates were simulated at various levels in order to evaluate the accuracy (by calculating the standard deviation of the percent error) of the estimated number of trips between any two stations after 50 simulations.

Based on the results (Table 17), the accuracy of the estimate increases as each of the variables increases (while holding the other variables constant). For example, for a proportion of $5 \%$ and a capture rate product of $56.3 \%$, the standard deviation is $5.5 \%$ for a traffic volume of 5,000 vehicles, and $3.8 \%$ for a traffic volume of 10,000 vehicles. Likewise, for a capture rate product of $90.3 \%$ and a traffic volume of 5,000 vehicles, the standard deviation is $1.8 \%$ for a proportion of $5 \%$ and $0.7 \%$ for a proportion of $25 \%$. Finally, for a proportion of $25 \%$ and a traffic volume of 1,000 vehicles, the standard deviation is $9.7 \%$ for a capture rate product of $25 \%$ and $4.8 \%$ for a capture rate product of $56.3 \%$

\subsection{Selecting an OD Technique}

The first step in preparing to conduct an OD study is to select a technique. In order to do this, the objectives of the study need to be considered, because different techniques yield different information, and the objectives of the study will themselves eliminate some of the techniques. The following paragraphs briefly describe the data obtained by each technique.

In the license plate matching technique, only the number of trips between $\mathrm{OD}$ pairs (observation stations) is collected, and the exact origins and destinations of the trips are unknown. Socioeconomic data about each vehicle driver is not collected because there is no interaction with the driver and no survey forms are used.

The license plate follow-up survey technique is a combination of various techniques. Like the license plate matching technique, license plates are recorded at various roadside stations. However, this technique also uses a mail-out/mail-in survey to collect detailed trip (true origins and destinations) and socioeconomic information about vehicle and driver. 
The vehicle intercept survey technique yields the same information as the license plate follow-up survey technique. However, this technique requires direct interaction with the driver, but also has much higher response rates. Therefore, this technique is generally better suited for low - medium speeds and low flow situations.

The vehicle tracing technique is similar to the license plate matching technique in that it collects strictly information on the number of vehicle trips between OD pairs. In addition, exact origins and destinations are obtained. This technique, however, does not yield socioeconomic data on the persons sampled. This technique is currently being used by only a few agencies on a large scale, and the results are not yet known.

\subsection{Selecting a Method}

The method chosen for the license plate matching, license plate follow-up survey, or the vehicle intercept survey techniques is largely based on the cost of each method and the desired accuracy, both of which are also tied to the traffic conditions of the road on which the data is being collected.

\subsubsection{Accuracy}

For the license plate matching technique, there are four basic methods for collecting license plate data: clipboard, audio, laptop, and video. In Chapter 6, capture rates for each of the manual methods (clipboard, audio, and laptop) were determined based on vehicle speed and flow. In addition, the relative amount of field recording and transcription error for each method was discussed. Chapter 5 evaluated the various types of equipment and the issues associated with each when collecting license plate data. Essentially, the video method should be used to achieve the most accurate results at higher speeds and flows. However, if less accuracy is acceptable or video is costprohibitive, manual methods can be used. The accuracy of the license plate matching technique was discussed in Chapter 7. Generally, as the study area increases (in terms of geography, population, and number of external stations) the proportions between stations decrease, which requires higher capture rates at each station.

If the license plate follow-up survey technique is used, license plate data has to be collected. As was done with the license plate matching technique, Chapter 6 evaluates the vehicle speeds and flows at which the video method should be chosen over manual methods for data collection. In addition, a survey has to be designed to 
obtain the trip information, and a method for distributing and conducting the survey has to be determined. While this was not evaluated as part of this project, the Travel Survey Manual discusses these issues in detail.

If the vehicle intercept survey technique is used, there are generally two practical methods available: roadside interviews or postcard questionnaires. Because postcard questionnaires require less time with the driver, this method should be used in low medium flow situations. However, because the interview method achieves a higher response rate and better accuracy, the two methods may be used in combination to avoid traffic delays. The number of interviews completed, however, is dependent on the number of interviewers on site and the flow of traffic into and out of the interview site.

The safety of the interviewers and drivers and the flow of traffic should not be compromised in an attempt to conduct more interviews.

OD studies often last 12 to 14 hours per day, beginning prior to the morning peak hour and continuing through the evening peak hour. Depending on the objectives of the study, the length of the study may be conducted over a shorter period. Caution should be used when adjusting OD data from short study periods (such as peak hour or partial day studies) to estimate hourly, daily, monthly, seasonal, or annual traffic patterns. Trends in OD travel patterns are more complex and difficult to detect and extrapolate than trends in traffic flow rates.

\subsubsection{Cost Items}

Generally, it is difficult to accurately estimate study costs of various OD techniques based on previous studies because of inflation, sampling rates (for survey methods), and incomplete information provided by those studies. However, Table 25 highlights the general cost items associated with each OD study technique. This information may be useful to determine the cost of a study or to compare two different techniques for the same study. 
Table 25: General Cost Items by OD Study Technique

\begin{tabular}{|c|c|c|c|}
\hline $\begin{array}{c}\text { License Plate } \\
\text { Matching }\end{array}$ & $\begin{array}{c}\text { License Plate } \\
\text { Follow-Up Survey }\end{array}$ & $\begin{array}{c}\text { Vehicle Intercept } \\
\text { Survey }\end{array}$ & Vehicle Tracing \\
\hline \hline Study Design & Study Design & Study Design & Study Design \\
Equipment & Equipment & Equipment & Equipment \\
Observers & Observers & Personnel & Probe Data \\
Training & Training & Training & Data Reduction \\
Data Reduction & BMV Fees & Police Assistance & \\
& Survey Design & Traffic Control & \\
& Survey Printing & Survey Design & \\
& Survey Mailing & Survey Printing & \\
& Data Reduction & Survey Mailing & \\
& & Data Reduction & \\
& & & \\
\hline
\end{tabular}

In the table above, "study design" refers to planning of the study (selecting the technique and method and determining the sites and the amount of personnel required at each site). "Equipment" refers to that required for field data collection (including safety equipment, recording equipment, communications equipment, etc.). "Observers" refer to the cost associated with the number of observers and the length of the study period. "Training" refers to any instruction given to the study staff prior to the actual data collection period. "Data reduction" refers to the amount of time required to transfer raw data into a form that can be analyzed. There are other costs, such as travel and per diem, that should be the same for all of the studies (except vehicle tracing).

Some license plate studies conducted by consultants in Indiana in recent years have ranged from $\$ 25,000$ to $\$ 50,000$, depending on the method used, the number of observation sites, and the amount of traffic (number of license plates) recorded. For vehicle intercept surveys, cost is often specified in terms of completed surveys, with studies ranging anywhere from $\$ 10$ to $\$ 50$ per completed survey. However, this value is sensitive to a variety of factors, particularly, the size of the staff involved in conducting the survey.

\subsection{Conclusions and Future Research}

This report offers descriptions, discussion, and analysis of various data collection techniques and methods for conducting Roadside Station Origin-Destination Studies. It evaluates the methods available for license plate data collection under various traffic conditions. This report also analyzes the accuracy of the License Plate Matching and Vehicle Tracing Techniques. Finally, it provides some guidelines for selecting the 
technique and data collection method, depending on the objectives of the study and traffic conditions of the roads in the study area.

In order to ensure the reliability of OD study results, better sample size formulas, using the information in Chapter 7, should be developed, particularly for the license plate matching technique, that incorporate traffic volume and capture rates at the stations and proportion of trips between stations. As was shown in the Chapter 7 of this report, these variables may cause the estimates to be inaccurate.

In addition, emerging technologies, such as vehicle tracing through wireless phones, have the potential to provide data for a wide range of applications in transportation beyond origin-destination data without time-consuming and costly field data collection. However, there are still issues, such as privacy, cost, and data reliability, which need to be addressed in order for this technology to become an accepted, adaptable, and efficient source of data. 


\section{LIST OF REFERENCES}

1. Asakura, Y., E. Hato, and M. Kashiwadani. Origin-Destination Matrices

Estimation Model Using Automatic Vehicle Identification Data and its Application to the Han-Shin Expressway Network. Transportation. Kluwer Academic Publishers, Netherlands, 2000, pp. 419-438.

2. Bonsall, P.W. and J. McKimm. Non-Response Bias in Roadside Mailback

Surveys. Traffic Engineering \& Control, v34, n 12, London, Dec 1993, pp. 582-591.

3. Cellular Telecommunications \& Internet Association.

http://www.ctia.org/research statistics/index.cfm/AID/10030. July 2005.

4. Der Wann, C. and Y.M. Chen. "Mobile Location Tracking with Velocity

Estimation." The IEEE $5^{\text {th }}$ International Conference on Intelligent

Transportation Systems, pp. 566-571.

5. Easycamcorders.com. http://www.easycamcorders.com/content/Beginners-

Guide.htm. June 2005.

6. Federal Communications Commission. http://www.fcc.gov/911/enhanced/. May 2005.

7. Florida State Patrol,

http://www.fhp.state.fl.us/html/FHPInTheNews/StopSurvey.htm. April 2005.

8. Fricker, J.D. and R.K. Whitford. Fundamentals of Transportation Engineering; a Multimodal Approach. Pearson Prentice Hall, Upper Saddle River, New Jersey, 2004.

9. Gupta, R., J.D. Fricker, and D.P. Moffett. Reduction of Video License Plate Data.

Transportation Research Record 1804. TRB, National Research Council, Washington, DC, 2002, pp. 31-38.

10. Hajek, J.J. Optimal Sample Size of Roadside Interview O-D Surveys. Ontario Ministry of Transportation and Communication. Ontario, Canada, Jan. 1977. 
11. MacDonald, K. "Introduction to Video 101." PULNiX America, Inc.

http://hexagon.physics.wisc.edu/research/technical\%20info/pulnixvideoguide. pdf, 2005.

12. Manual of Transportation Engineering Studies. Robertson, H.D, Editor, J.E. Hummer, and D.C. Nelson. Institute of Transportation Engineers, Washington, DC, 1994.

13. Martin, P.T., and M.C. Bell. Vehicle Tracking Through Unsampled RegistrationPlate Observation. Traffic Engineering and Control, v34, n 1, London, Jan 1993, pp. 8-12.

14. Nicholson, David. "Index of US License Plate Pages."

http://www.15q.net/usindex.html. July 2005.

15. Oh, C. and S.G. Ritchie. Anonymous Vehicle Tracking for Real-Time Traffic Surveillance and Performance on Signalized Arterials. Transportation Research Record 1826. TRB, National Research Council, Washington, DC, 2003, pp. 37-44.

16. OnStar. http://www.onstar.com/us english/jsp/index.jsp. May 2005.

17. Plotnikov, M. and P.W. Shuldiner. Effects of Ambient Light, Camcorder Settings, and Automated License Plate Reader Settings on Plate Transcription Rates. Transportation Research Record 1804. TRB, National Research Council, Washington, DC, 2002, pp. 56-61.

18. Quiroga, C., R. Henk, and M. Jacobson. Innovative Data Collection Techniques for Roadside Origin-Destination Surveys. Transportation Research Record 1719. TRB, National Research Council, Washington, DC, 2000, pp. 140-146.

19. Schaefer, M.C. "License Plate Matching Surveys: Practical Issues and Statistical Considerations." ITE Journal, Institute of Transportation Engineers, Washington, DC, July 1988, pp. 37-42.

20. Shuldiner, P.W., S.A. D'Agostino, and J.B. Woodson. Determining Detailed Origin-Destination and Travel Time Patterns Using Video and Machine Vision License Plate Matching. Transportation Research Record 1551. TRB, National Research Council, Washington, DC, 1996, pp. 8-17. 
21. Slavik, M.M. Errors in Origin-Destination Surveys Done by Number-Plate Techniques. Transportation Research Record 1050. TRB, National Research Council, Washington, DC, 1985, pp. 51-58.

22. Stokes, R.W. and T. Chira-Chivala. Design and Implementation of Intercity Origin-Destination Surveys. Transportation Research Record 1236, TRB, National Research Council, Washington, DC, 1989.

23. The Highways Agency, The Scottish Office Development Department, The Welsh Office, and The Department of the Environment for Northern Ireland. "Traffic Surveys by Roadside Interview." http://www.archive2.officialdocuments.co.uk/ document/deps/ha/dmrb/vol5/section1/ta1181a.pdf. United Kingdom, 2005.

24. Travel Survey Manual. Cambridge Systematics, Inc. Travel Model Improvement Program, USDOT Federal Highway Administration, Washington, DC, 1996.

25. Travel Time Data Collection Handbook. Turner, S.M., W.L. Eisele, R.J. Benz, and D.J. Holdener. Texas Transportation Institute, College Station, TX, 1998.

26. Trimble. http://www.trimble.com/gps/index.html. July 2005.

27. Turner, S.M. Advanced Techniques for Travel Time Data Collection.

Transportation Research Record 1551. TRB, National Research Council, Washington, DC, 1996, pp. 51-58.

28. Virkud, U. and C.S. Keyes. Design and Implementation of a Statewide Roadside Origin-Destination Survey in Vermont. Transportation Research Record 1477. TRB, National Research Council, Washington, DC, 1995, pp. 15-25.

29. Wang, C.P. and S. Nallamothu. Basis of License Plate Recognition and a New Approach. Congress on Computing in Civil Engineering, Proceedings, 1997, pp. 143-152.

30. Washburn, S.S. and N.L. Nihan. Using Voice Recognition to Collect License Plate Data for Travel Time Studies. Transportation Research Record 1593. TRB, National Research Council, Washington, DC, 1997, pp. 41 - 46.

31. Welby, Marc J. “The License Plate Shack.” http://www.plateshack.com. July 2005.

32. Yahoo. "Missouri May Track Cell Phones for Traffic Data." http://news.yahoo.com/s/ap/monitoring motorists, October 2005. 
APPENDIX A

SURVEY OF STATE DOTS 


\section{Survey of State DOTs on Origin-Destination Studies March 2005}

The Indiana Department of Transportation, in conjunction with Purdue University, is performing research in order to develop guidelines for conducting origin-destination studies in the state of Indiana. Attached is a brief survey for Planning or Data \& Statistics personnel regarding your state DOT's policies and experiences in data collection for recent OD studies. The types of questions are short answer or option selection. Please complete the survey or forward on to the appropriate person in your agency. If you have any questions about the survey, please email bpquy@purdue.edu. Your participation is important and much appreciated.

1. Does your state DOT have any written guidelines for conducting origin-destination studies (for cities and towns located outside the jurisdiction of an MPO)? If so, how could I obtain a copy of those guidelines?

Answer:

2. Does your state DOT have a preferred data collection method for conducting origin-destination studies (whether completed in-house or by a consultant)? If so, what is that method?

Answer:

3. If your state DOT or its consultants have conducted any origin-destination studies in the last five years, please complete sections $\mathrm{a}-\mathrm{f}$ below. If not, skip to question 6 .

a. Roadside License Plate Recording \& Matching Technique - requires license plates to be recorded at multiple stations, transcribed into software, and matched between stations.

i. How many studies have been conducted in the last five years using this technique? If zero, skip to part b. Answer:

ii. Please indicate the method in which license plates were recorded and transcribed for these studies. Indicate all that apply.

_ Paper and pencil (with manual transcription of license plate numbers into software for matching between recording stations) Audio tape (manual transcription)

- Audio tape (automatic voice recognition technology for transcription)

- Laptop computer (bypassing transcription step)

— Video (manual transcription)

- Video (automatic character recognition technology for transcription)

— Other (please specify):

b. Roadside License Plate Recording \& Follow-Up Survey Technique - requires the motor vehicles department to provide addresses and/or phone numbers for each license plate identified at a roadside station after which the vehicle owner is contacted in order to obtain information about the trip at the time the license plate was recorded.

i. How many studies have been conducted in the last five years using this technique? If zero, skip to part c. Answer:

ii. Please indicate the method in which license plates were recorded and transcribed for these studies. Indicate all that apply.

_ Paper and pencil (with manual transcription of license plate numbers for the DMV to provide mailing addresses to vehicle owners)

Audio tape (manual transcription)

- Audio tape (automatic voice recognition technology for transcription)

_ Laptop computer (bypassing transcription step)

_ Video (manual transcription) 
_ Video (automatic character recognition technology for transcription)

_ Other (please specify):

iii. Please indicate how the vehicle owners were contacted. Indicate all that apply.

— Telephone Interview

- Mail-out/Mail-back Survey

— Other (please specify):

c. Vehicle Intercept Survey Technique - stops some or all drivers traveling on a roadway and seeks information about their current trip.

i. How many studies have been conducted in the last five years using this technique? If zero, skip to part iii. Answer:

ii. Please indicate the method by which these vehicle intercept surveys were conducted. Indicate all that apply.

_ Roadside Interviews (collecting information directly from the driver)

- Postcard Survey (distributed to drivers on roadway to be completed and mailed back) _ Other (please specify):

iii. If Vehicle Intercept Surveys are not used, why not? Answer:

d. Travel Diary Technique - recruits a random sample of people to record all trips (automobile mode only or all modes) for a period of usually one or two days.

i. How many studies have been conducted in the last five years using this technique? If zero, skip to part e. Answer:

ii. Please indicate the method these travel diaries were recorded. Indicate all that apply.

_ Manual recording (paper \& pencil)

- Manual recording (electronic device such as a personal data assistant)

- Automatic recording (GPS)

_ Other (please specify):

e. Recall Interview/Survey Technique - surveys a random sample of people about all trips one day prior to the survey. Unlike the travel diary technique, the sample does not know of the survey in advance.

i. How many studies have been conducted in the last five years using this technique? If zero, skip to part f. Answer:

ii. Please indicate the method by which these recall surveys/interviews were conducted. Indicate all that apply.

_ Telephone interview

- Mail-out/Mail-back survey

— Other (please specify):

f. Vehicle Tracing Technique. This technique unobtrusively traces driver's paths with or without their knowledge or permission. 
i. How many studies have been conducted in the last five years using this technique? If zero, skip to question 4. Answer:

ii. Please indicate the method these vehicle traces were conducted. Indicate all that apply.

_ Cell phone tracing

_ GPS tracing

— Other (please specify):

4. Is there any particular data collection method (not limited to those listed above) your state DOT has utilized that has met or exceeded your expectations in terms of time, cost, accuracy, etc? Please explain.

Answer:

5. Are there any methods that have failed to meet your expectations? Please explain. Answer:

6. Please return the survey via email to bpguy@purdue.edu. Thank you for your time and cooperation.

Sincerely,

Bryan P. Guy

Graduate Research Assistant

Purdue University

School of Civil Engineering

550 Stadium Mall Drive

West Lafayette, IN 47907

(402) 616-1143 bpguy@purdue.edu 
APPENDIX B

GUIDELINES FOR CONDUCTING LICENSE PLATE SURVEYS 


\section{PROJECT SUMMARY REPORT}

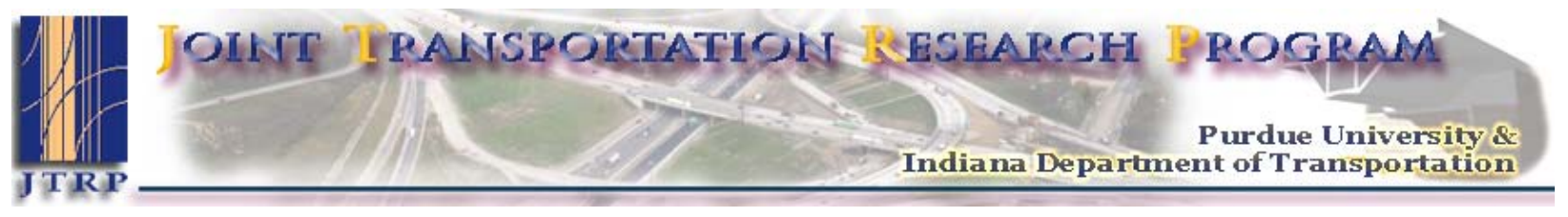

Project Indiana SPR-2935

July 2007

Development of Standards for Conducting an Origin-Destination Study

Authors: Bryan Guy and Jon Fricker

\section{Guidelines for Conducting License Plate Surveys}

Origin-Destination (OD) studies are conducted to gain an understanding of the traffic patterns in or through a particular area of interest during a particular period of time. During our comprehensive examination of OD study methods, it became apparent that the limitations of human observers were just as important as the capabilities of the technologies that could be chosen. A series of measurements, calculations, and simulations were carried out to determine the conditions under which roadside license plate data collection methods such as Clipboard, Audiotape, Laptop, and Video cameras would be most appropriate. Conditions such as traffic volumes, traffic speeds, and observer visual acuity can affect the speed and accuracy with which identification and recording of

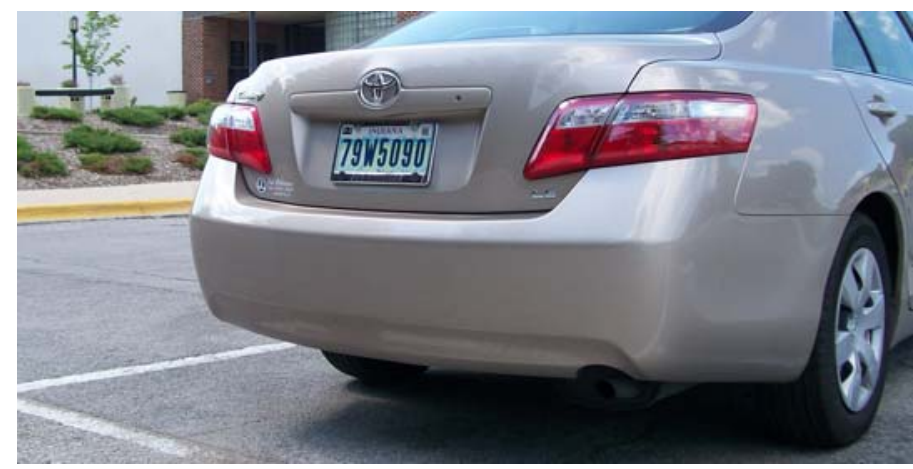

Figure 1. Recent standard Indiana license plate license plates can be accomplished. These factors influence the capture rate for license plates, which in turn determines the number of vehicles that need to be observed to produce an externalexternal OD matrix with sufficient accuracy. This Project Summary presents guidelines for the planning and conduct of a license plate survey, especially a video-based survey intended for license plate matching.

\section{DATA COLLECTION METHODS}

Brief descriptions of the most common ways to record license plate strings are given here. See Guy and Fricker (2005) for more methods and details, including the advantages and disadvantages of each method. 


\section{The Clipboard Method}

Observers at each station manually record license plate data with paper and pencil. Time stamps should also be recorded -- ideally every minute, but not greater than five minutes. According to Martin and Bell (1993), observers can record approximately 170 full license plates per hour or 800 partial license plates per hour (three or four digits). Simultaneous traffic counts should be conducted in order to obtain an accurate capture rate (which is used later for data expansion). These written records must be manually transcribed into electronic files to facilitate the matching step.

\section{The Audio Method}

In this method, observers speak the data into some audio recording device. According to Martin and Bell (1993), between 1000 and 1200 license plates per hour can be recorded by one observer. However, even at $200 \mathrm{vph}$, vehicles can bunch together closely enough to overwhelm even the most dedicated observer several times an hour. Transcription typically takes two to three hours for every hour of tape (Turner 1998), but voice-actuated recorders can reduce the transcription ratio to $1: 1$.

\section{The Laptop Method}

In this method, the observer at each station types the data into a laptop computer. The character strings are stored along with an exact time stamp (to the nearest second) assigned by the computer after the entry is completed. As with the clipboard and audio methods, traffic volume counts must be conducted to determine the number of missed vehicles. Unlike the other methods (clipboard, audio, video, or photo), the laptop method does not require a subsequent transcription step, because the license plate strings and time stamps are entered directly into the computer in the field. Nine hundred 4-character strings can be recorded per hour with this method (Turner 1998).

\section{The Video Method}

This method allows observers to record the license plates with a camcorder. The video tapes are then reviewed in the office, where license plate numbers, time stamps, and vehicle classification (if desired) are transcribed. Traffic counters on the road determine the number of missed vehicles when the video camera is not operating, because of tape change, battery replacement, or malfunction.

\section{LICENSE PLATE IDENTIFICATION}

\section{Maximum Flow Rate for Manual Methods}

Vehicles registered in Indiana receive only one plate, which is mounted on the rear of the vehicle. (See Figure 1.) The research project sought to specify a flow rate at which the video method should be used instead of manual methods, because at higher flow rates, an unacceptably high percentage of license plates will be missed. Figure 2 shows the expected percent of vehicles whose plates captured can be estimated for any flow rate. (See Guy and Fricker 2005 for details.) If the capture rate is sufficient for the purpose of the study, that method can be used. If not, however, the video method is recommended. 


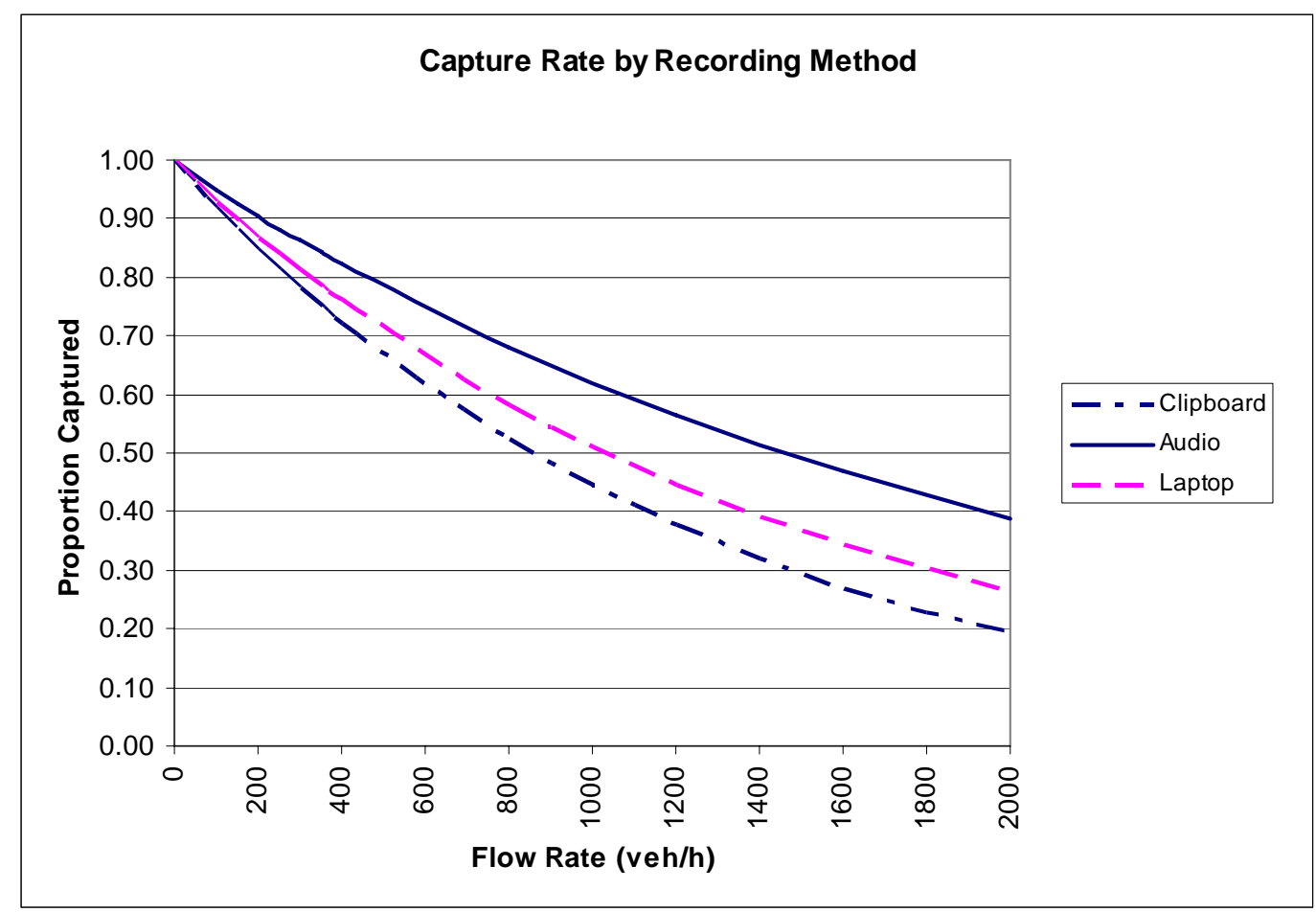

Figure 2. Capture Rates by Vehicle Flow for each Non-Video Method

Table 1 summarizes the maximum flow rates for 95\%, 85\%, and 75\% 4-character capture rates (rounded to the nearest 25 vehicles). To record 4 characters on 95 percent of all identifiable license plates using the audio method, the flow rate should not exceed $100 \mathrm{vphpl}$. If a lower capture rate is acceptable, the maximum flow rates increase significantly. For flow rates that exceed those shown in the table, the video method is recommended for recording license plates.

Table 1. Max Uninterrupted Flow Rate (vphpl) for Manual Recording Methods

\begin{tabular}{|l|c|c|c|}
\hline Method & 95\% Capture & 85\% Capture & 75\% Capture \\
\hline \hline Clipboard & 50 & 200 & 350 \\
\hline Audio & 100 & 350 & 650 \\
\hline Laptop & 75 & 250 & 450 \\
\hline
\end{tabular}

\section{ERRORS IN LICENSE PLATE DATA}

\section{Field and Transcription Errors}

It is unlikely that an observer will make no mistakes in recording the data in the field. Field errors are errors that are made as the license plate strings are identified and recorded from the passing vehicle onto the clipboard, audio tape, laptop, or videotape. Transcription errors are errors that are made while transferring the data in their raw form into some database. In Table 2, the total amount of error for any particular method after field recording and transcribing the data is the sum of the two values shown. These errors, in effect, reduce the capture rates even further, and must be considered when expanding the sample data to the entire population. The 4character recording error for all methods is generally less than the full string recording error. 
Table 2 Field \& Transcription Errors by Method

\begin{tabular}{|l|c|c|}
\hline Method & Field & Transcription \\
\hline \hline Clipboard & $0.0 \%$ & $0.0 \%$ \\
\hline Audio & $3.0 \%$ & $1.5 \%$ \\
\hline Laptop & $2.9 \%$ & n/a \\
\hline Video & n/a & $0.3 \%$ \\
\hline
\end{tabular}

When these errors occur, there is the possibility (for the license plate matching technique) that some license plates that should be matched to another location will not be matched. Therefore, the number of matches needs to be increased to reflect these errors.

\section{Transcription Time}

The total transcription time for each of the methods was measured from the same license plate data used to determine errors. In Table $3, n$ is the number of license plates and $L$ is the total duration (in seconds) of audio or video tape recorded in the field. Generally, the video method has the highest transcription time. However, it must be noted that video also has higher capture rates and lower errors than the other methods.

Table 3 Manual Transcription Time (sec) by Method

\begin{tabular}{|l|c|}
\hline Method & Time \\
\hline \hline Clipboard & $2.4 n$ \\
\hline Audio & $L+0.3 n$ \\
\hline Laptop & n/a \\
\hline Video & $L+3.5 n$ \\
\hline
\end{tabular}

\section{CHECKLIST FOR PLANNING AND CONDUCTING A VIDEO LICENSE PLATE SURVEY}

The success of a video license plate survey depends on avoiding mistakes and paying attention to detail. If a license plate is observed and recorded at one station, but is missed at another station, that is a trip that cannot be matched for inclusion in the OD matrix that is the purpose of the study. We present below a list of steps that will improve the chances for success.

\section{Preparation}

1. Establish the reason for the license survey. This will guide your decisions as to the duration of the study and the location of stations at which to observe license plates.

2. Visit the proposed locations of stations. Find specific locations for cameras and attendants that provide the proper camera setup while preserving the safety of the attendants. Provide at least 12 feet of space between high-speed vehicles and the attendants. Look for possibilities for glare or bright backlighting and consider remedies. Take photographs that clearly show the exact location of each site.

3. Contact the local jurisdictions to coordinate the placement of traffic counters at each station.

4. Notify law enforcement agencies about your plans.

5. Prepare packets for each station: tapes marked with station and sequence (" $1{ }^{\text {st }}$ tape", " 2 nd tape”, etc.), batteries or power inverters, map and directions to assigned station, photo of exact station location, instructions for camera setup, cell phone number for supervisor(s). 
6. Acquire appropriate equipment and supplies.

A. Digital camcorders with sufficient optical zoom (at least 10x) and shutter speed (1/1000 or faster). If all the camcorders are the same, training and supervision will be greatly simplified. We have found camcorders that meet our requirements can be purchased for under $\$ 350$ each.

B. Power supplies (batteries) with a "life" at least as long as the tapes to be used. When tapes and/or batteries are being replaced, license plates are being missed. When all cameras are the same, replacement batteries can be provided much more easily. If vehicles can be parked near the cameras, power inverters can be used to eliminate the need for camera batteries.

C. Tripods that can resist the wind forces of large vehicles passing by. We have found tripods at $\$ 30$ each that are more than adequate.

7. Train your camera attendants in how to set up the camera. At a session held in a "classroom" shortly before going into the field, have the attendants practice mounting camera on tripod, adjusting zoom and shutter speed, displaying correct date and time, and quickly replacing battery and tape. Show a video (or PowerPoint) presentation of good (and perhaps some bad) examples of video license plate images. Get the cell phone number of each attendant and synchronize watches. The key elements in setting up the camcorder are:

A. Adjust the tripod so that the camera is between knee and waist height. (See Figure 3.) This creates a better angle for recording license plates and avoids making the camera/tripod "top heavy". Tilt the camera so that license plates that are in normal and higher-than-normal locations on vehicles can be seen.

B. Do not put the camera closer than 12 feet from a high-speed traveled lane. (See Figure 3.) This minimizes the probability that a large truck will blow the camera over and provides a good margin of safety for the observer. (A smaller distance can be used along a deceleration lane just before a turn, where the speed of a passing vehicle is low enough to allow reaction by driver and attendant.) See Figure 4.

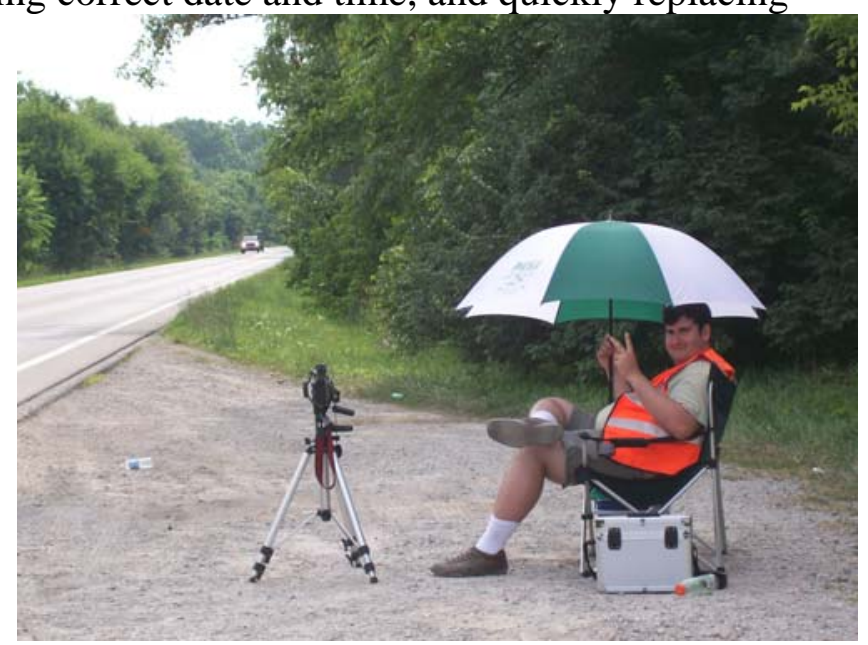

Figure 3. Video station along US highway

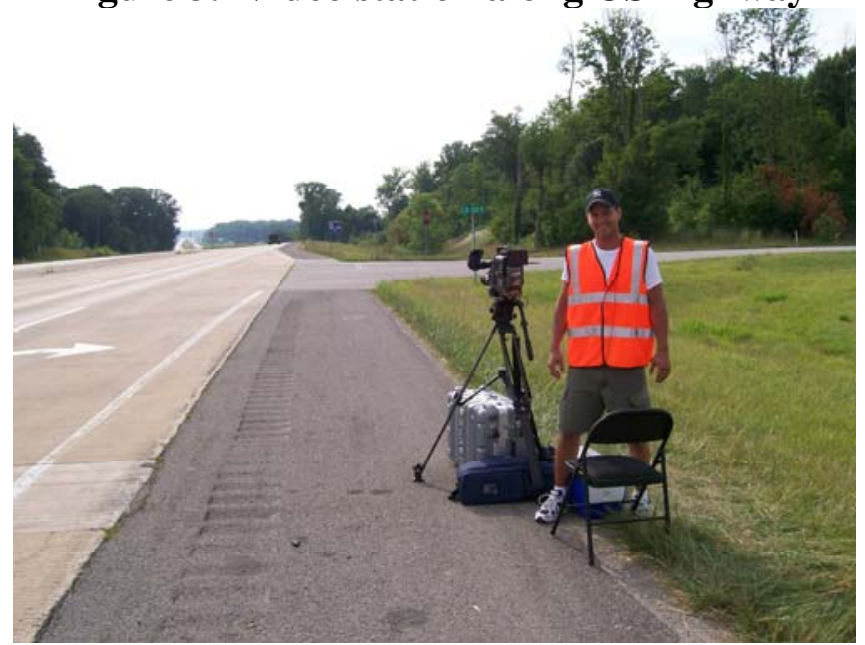

Figure 4. Video station along deceleration lane

C. If we define a 90-degree shooting angle as straight across the road and a zero-degree shooting angle as parallel to the road, point your camera so that your shooting angle is about 30 degrees from parallel. See Figures 3 and 4. 
D. Zoom your camera's lens so that the rear of the vehicles you are recording fill about $2 / 3$ the width of the frame. (See Figures 5-7.) If you are recording two lanes in the same direction, this fraction may be a little less than $2 / 3$ for the far (left) lane vehicles and more like $3 / 4$ for the near (right) lane vehicles. Even at this close-in zoom setting, the license on each vehicle will be visible for several frames during playback, and it will be much easier to read the letters/numbers during playback.

E. Make sure the camera's shutter speed is between 1/1000 and 1/4000. At slower speeds, the license plate image will be blurred.

\section{During video data collection}

8. During the data collection, have supervisors visit each station on a frequent basis.

Supervisors should:

A. Check camera setup for tilt, shutter speed, zoom, shooting angle, etc.

B. Check status of battery and tape.

C. Have ready at least one spare camera, one spare battery, and a set of video tapes.

\section{After data collection}

9. After data collection, train the staff who will transcribe video images into data files.

A. Should the all license characters in the video images be converted into an entry in the data file? What about characters that are small, such as the letters in Indiana's new In God We Trust plates (Figure 5), the last letter on pickup plates (Figure 6), or the small letter in the middle of many Indiana plates (Figure 7)? We recommend entering all large (2.75-inch) characters, while omitting the smaller (about 1.25 inches) characters, which often cannot be seen, even on videotape.

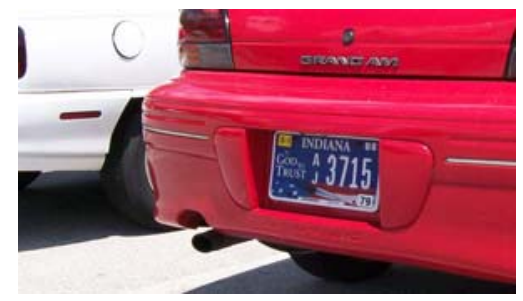

Figure 5. In God We Trust

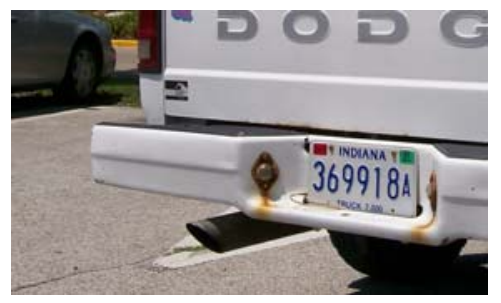

Figure 6. Pickup Truck

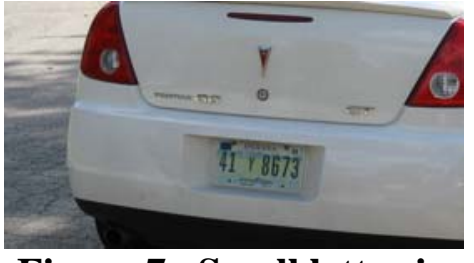

Figure 7. Small letter in recent standard format

B. Because even 4 digits may not be enough to distinguish some vehicles from others, we advocate adding other information to the data entry line. This will make data entry slower, but greatly assist the matching process. Table 4 contains excerpts from a data file created from a video license plate survey of northbound vehicles at Station 6 and sorted on the Characters column. It has a column for Vehicle Type, which prevented a false

\begin{tabular}{|l|r|l|l|r|r|r|}
\hline \multicolumn{5}{|c|}{ Table 4. Excerpt from license plate data file for Station 6NB } \\
\hline Veh Type & Characters & $?$ & Comments & Hr & Min & Sec \\
\hline suv & 118 & & 25Z cou; tan & 3 & 40 & 52 \\
\hline car & 118 & & Buick; 9E cou? & 3 & 43 & 2 \\
\hline pu & 1934 & $?$ & IGWT & 5 & 25 & 22 \\
\hline minivan & 1939 & & special plate & 4 & 31 & 37 \\
\hline car & 1942 & & 52 cou & 4 & 53 & 24 \\
\hline suv & 1952 & & & 5 & 41 & 44 \\
\hline car & 1993 & & special plate; plum? & 5 & 52 & 36 \\
\hline car & 1993 & & 9A cou; cream & 3 & 52 & 22 \\
\hline
\end{tabular}


match of an SUV and a car, both of which had a plate that ended with "118". The Comments column prevented a false match of two cars with "1993". They had different colors, and one was a special plate (such as in Figure 8) while the other had a standard format from County 9. The "?" column alerts the analyst doing the matching that the transcriber was not certain of a character. A good guess in column 2 with a "?" in column 3 is much better than entering a partial string or no string at all. The recommended entries for Figures 1, 5-8 in the "Character" column of an improved Table 4 (see Table 5, produced after a review of the video tapes) would be “79W5090”, “3715”, “369918”, “418673”, and “3244”. The smaller characters are ignored during data transcription. The full data file can now be re-sorted.

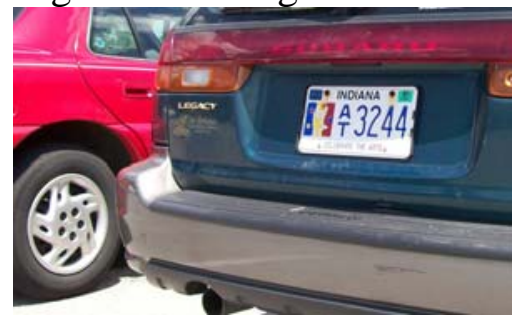

Figure 8. Special plate to support The Arts

\begin{tabular}{|l|r|l|l|r|r|r|r|}
\hline \multicolumn{7}{|c|}{ Table 5. Improved license plate data file for Station 6NB } \\
\hline Veh Type & Characters & $?$ & Comments & $\mathrm{Hr}$ & Min & Sec & Sta ID \\
\hline suv & $25 Z 118$ & & 25Z cou; tan & 3 & 40 & 52 & $6 \mathrm{NB}$ \\
\hline car & 9118 & & Buick; 9E cou? & 3 & 43 & 2 & $6 \mathrm{NB}$ \\
\hline pu & 1934 & $?$ & IGWT, Dodge & 5 & 25 & 22 & $6 \mathrm{NB}$ \\
\hline minivan & 1939 & & special plate & 4 & 31 & 37 & $6 \mathrm{NB}$ \\
\hline car & 521942 & & 52 cou & 4 & 53 & 24 & $6 \mathrm{NB}$ \\
\hline suv & 81952 & & 8 cou & 5 & 41 & 44 & $6 \mathrm{NB}$ \\
\hline car & 1993 & & special plate; plum? & 5 & 52 & 36 & $6 \mathrm{NB}$ \\
\hline car & $9 \mathrm{~A} 1993$ & & 9A cou; cream & 3 & 52 & 22 & $6 \mathrm{NB}$ \\
\hline
\end{tabular}

11. Match license plates in the data file. Before matching, add a Station ID column to the data file. Whether you use a spreadsheet or more specialized software, check apparent matches using plausible travel times between stations. (Moffett and Fricker 1993) A through trip that takes too long may have been an external-internal trip, followed by an internal-external trip. Also, having video tapes with time stamps allows you to confirm or discard any questionable matches. (See Table 4.)

12. Use data from traffic counters at each station to verify the traffic flows during the video observations and help estimate AADT from 48-hour counts. Because heavy vehicles are often a major reason for a video license plate trace, getting vehicle classification data is very useful. Recent experience has indicated that most semi-trailers have only one plate - located at the lower left corner or bottom center of the rear of the semi-trailer. This plate is usually legible on video tape. The days of multiple license plates on trailers seem to be gone.

\section{REFERENCES}

1. Guy, Bryan P. and Jon D. Fricker, Evaluation of Data Collection Techniques and Methods for Roadside Station Origin-Destination Studies, Draft Report, School of Civil Engineering, Purdue University, West Lafayette, Indiana, December 2005.

2. Martin, P.T., and M.C. Bell, "Vehicle Tracking Through Unsampled Registration-Plate Observation”, Traffic Engineering and Control, vol 34, nr 1, London, Jan 1993, pp. 8-12. 
3. Moffett, David P. and Jon D. Fricker, "Improved License Plate Matching Procedures for Estimation of Origin-Destination Matrices”, Proceedings, Fourth National Conference on Transportation Planning Applications, Daytona Beach, Florida, 5 May 1993.

4. Turner, S.M., W.L. Eisele, R.J. Benz, and D.J. Holdener, Travel Time Data Collection Handbook, Texas Transportation Institute, College Station, TX, 1998. 